\title{
NON-UNIPOTENT CHARACTER SHEAVES AS A CATEGORICAL CENTRE
}

\author{
G. LUSZTIG \\ Department of Mathematics, M.I.T., Cambridge, MA 02139, USA. \\ E-mail: gyuri@math.mit.edu \\ \|\| \\ $\|$ \\ Abstract \\ Let $G$ be a reductive connected group over an algebraic closure of finite field. In \\ this paper we give the classification of character sheaves on $G$ in categorical terms (as \\ a categorical centre). Previously such a classification was known for unipotent character \\ sheaves and in the case where the ground field is replaced by the complex numbers.
}

\section{Introduction}

0.1. Let $\mathbf{k}$ be an algebraically closed field of characteristic $p \geq 0$ and let $G$ be a reductive connected group over $\mathbf{k}$. We fix a prime number $l$ different from $p$. The theory of character sheaves developed in [14] and its sequels associates to $G$ a collection of simple perverse $\overline{\mathbf{Q}}_{l}$-sheaves on $G$ which in many respects mimic the irreducible representations of the finite Chevalley groups of the same type as $G$. The classification of character sheaves was given in [17]. A few years ago, Bezrukavnikov, Finkelberg and Ostrik [2] gave a less computational (and more categorical) approach to the classification of character sheaves assuming that the centre of $G$ is connected and that $p=0$. For applications to the study of finite Chevalley groups it was desirable to include the case when $p>0$, but it was not clear how to do that by the method of [2] which relied on certain results on Harish-Chandra modules that are not available when $p>0$. In [24], I found a way to obtain the

Received June 16, 2015 and in revised form August 19, 2016.

AMS Subject Classification: 20G99.

Key words and phrases: Character sheaf, flag manifold, local system, reductive group.

Supported in part by NSF grant DMS-1303060. 
classification of unipotent character sheaves in categorical terms assuming that $p>0$, using a functor (truncated restriction) whose definition was different from that in [2]; moreover, in [25], I extended this to a classification of unipotent representations of a finite Chevalley group in categorical terms. In this paper I will extend the method of [24] to obtain the classification of not necessarily unipotent character sheaves of $G$ in categorical terms assuming that $p>0$.

0.2. Notation. In the rest of this paper $\mathbf{k}$ is an algebraic closure of the finite field $\mathbf{F}_{q}$ with $q$ elements. All algebraic varieties are over $\mathbf{k}$. We denote by $\mathbf{p}$ the algebraic variety consisting of a single point. For an algebraic variety $X$ we write $\mathcal{D}(X)$ for the bounded derived category of constructible $\overline{\mathbf{Q}}_{l}$-sheaves on $X$. Let $\mathcal{M}(X)$ be the subcategory of $\mathcal{D}(X)$ consisting of perverse sheaves on $X$. For $K \in \mathcal{D}(X)$ and $i \in \mathbf{Z}$ let $\mathcal{H}^{i} K$ be the $i$-th cohomology sheaf of $K$ and let $K^{i}$ be the $i$-th perverse cohomology sheaf of $K$; if $x \in X$, let $\mathcal{H}_{x}^{i} K$ be the stalk of $\mathcal{H}^{i} K$ at $x$. Let $\mathfrak{D}(K)$ be the Verdier dual of $K$. If $X$ has a fixed $\mathbf{F}_{q^{-}}$-structure $X_{0}$, we denote by $\mathcal{D}_{m}(X)$ what in $[1,5.1 .5]$ is denoted by $\mathcal{D}_{m}^{b}\left(X_{0}, \overline{\mathbf{Q}}_{l}\right)$.

Note that if $K \in \mathcal{D}_{m}(X)$ then $K$ can be viewed as an object of $\mathcal{D}(X)$ denoted again by $K$. If $K \in \mathcal{D}_{m}(X)$ is a perverse sheaf and $h \in \mathbf{Z}$, we denote by $g r_{h}(K)$ the subquotient of pure weight $h$ of the weight filtration of $K$. If $K \in \mathcal{D}_{m}(X)$ and $i \in \mathbf{Z}$ we write $K\langle i\rangle=K[i](i / 2)$ where [i] is a shift and $(i / 2)$ is a Tate twist; we write $K^{\{i\}}=g r_{i}\left(K^{i}\right)(i / 2)$. If $K$ is a perverse sheaf on $X$ and $A$ is a simple perverse sheaf on $X$ we write $(A: K)$ for the multiplicity of $A$ in a Jordan-Hölder series of $K$.

Assume that $C \in \mathcal{D}_{m}(X)$ and that $\left\{C_{i} ; i \in I\right\}$ is a family of objects of $\mathcal{D}_{m}(X)$. We shall write $C \approx\left\{C_{i} ; i \in I\right\}$ if the following condition is satisfied: there exist distinct elements $i_{1}, i_{2}, \ldots, i_{s}$ in $I$, objects $C_{j}^{\prime} \in \mathcal{D}_{m}(X)$ $(j=0,1, \ldots, s)$ and distinguished triangles $\left(C_{j-1}^{\prime}, C_{j}^{\prime}, C_{i_{j}}\right)$ for $j=1,2, \ldots, s$ such that $C_{0}^{\prime}=0, C_{s}^{\prime}=C$; moreover, $C_{i}=0$ unless $i=i_{j}$ for some $j \in[1, s]$. (See [20, 32.15].)

Let $\mathcal{A}=\mathbf{Z}\left[v, v^{-1}\right]$ where $v$ is an indeterminate. Let ${ }^{-}: \mathcal{A} \rightarrow \mathcal{A}$ be the ring homomorphism such that $\overline{v^{m}}=v^{-m}$ for any $m \in \mathbf{Z}$. If $f \in \mathbf{Q}\left[v, v^{-1}\right]$ and $j \in \mathbf{Z}$ we write $(j ; f)$ for the coefficient of $v^{j}$ in $f$. 
Let $\mathcal{B}$ be the variety of Borel subgroups of $G$. For any $B \in \mathcal{B}$ let $U_{B}$ be the unipotent radical of $B$. In this paper we fix $\mathbf{B} \in \mathcal{B}$ and a maximal torus $\mathbf{T}$ of $\mathbf{B}$; let $\mathbf{U}=U_{\mathbf{B}}$. Let $\nu=\operatorname{dim} \mathbf{U}=\operatorname{dim} \mathcal{B}, \rho=\operatorname{dim} \mathbf{T}, \Delta=\operatorname{dim} G=2 \nu+\rho$.

For any algebraic variety $X$ let $\mathfrak{L}=\mathfrak{L}_{X}=\alpha_{!} \overline{\mathbf{Q}}_{l} \in \mathcal{D}(X)$ where $\alpha$ : $X \times \mathbf{T} \rightarrow X$ is the obvious projection. When $X$ is defined over $\mathbf{F}_{q}, \mathfrak{L}$ is naturally an object of $\mathcal{D}_{m}(X)$.

Unless otherwise specified, all vector spaces are over $\overline{\mathbf{Q}}_{l}$; in particular all representations of a finite group $\Gamma$ are assumed to be in (finite dimensional)

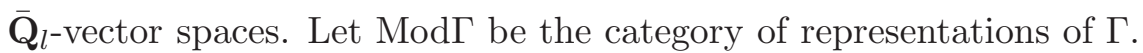

0.3. We now discuss the content of various sections in some detail. The main difference between [24] and the present paper is that the study of $G$ equivariant sheaves on $\mathcal{B} \times \mathcal{B}$ is replaced by that of monodromic sheaves that is, certain $G$-equivariant sheaves on $\tilde{\mathcal{B}}^{2}=G / \mathbf{U} \times G / \mathbf{U}$. The role that the Hecke algebra played in [24] is now played by a monodromic analogue $\mathbf{H}$ of the Hecke algebra which was introduced (as an endomorphism algebra of the representation of a Chevalley group over $\mathbf{F}_{q}$ induced by the unit representation of a Sylow $p$-subgroup) by Yokonuma [30] in 1967. In Section 1 we recall from [20] various notions for $\mathbf{H}$ that were known earlier for ordinary Hecke algebras: the canonical basis, the left cells, the two-sided cells, the $a$-function, the asymptotic version. (Something close to the canonical basis of $\mathbf{H}$ and its connection to intersection cohomology was already discussed in [13, Ch.1].) A key role in our discussion is the fact (see [20]) that $\mathbf{H}$ is a matrix ring over an ordinary extended Hecke algebra. In Section 2 we study the $G$-equivariant sheaves on $\tilde{\mathcal{B}}^{2}$ with monodromy of finite order dividing a fixed number $n$; we define truncated convolution of such sheaves, see 2.24. This differs from the non-monodromic case since it now involves direct images with compact support of non-proper maps, which makes the analysis more complicated. In this section and in the subsequent ones we refer several times to two technical lemmas [24, 1.12] and [24, 8.2] but we apply them in various cases which, although not explicitly contained in those references, are proved just as in the references. In Section 3 we define truncated convolution of $G$-equivariant sheaves on $Z=\mathbf{T} \backslash \tilde{\mathcal{B}}^{2}$ with monodromy of order dividing $n$. Most of this section is concerned with the study of a functor $\mathfrak{b}$ (see 3.13) from sheaves on $Z$ to sheaves on $\tilde{\mathcal{B}}^{2}$ and its truncated version. In Section 4 we discuss the unit object and rigidity of the truncated 
monoidal category $\mathcal{C}^{\mathbf{c}} \tilde{\mathcal{B}}^{2}$ of sheaves on $\tilde{\mathcal{B}}^{2}$ associated to a two-sided cell $\mathbf{c}$ in H. In Section 5 we define truncated induction from a certain category of sheaves $\mathcal{C}^{\mathbf{c}} Z$ on $Z$ associated to a two-sided cell $\mathbf{c}$ of $\mathbf{H}$ to a certain category of sheaves $\mathcal{C}^{\mathbf{c}} G$ on $G$ associated to $\mathbf{c}$ and we define truncated restriction going in the opposite direction. We also define truncated convolution in $\mathcal{C}^{\mathbf{c}} G$. In Section 6 we show (Theorem 6.13) that truncated restriction provides an equivalence of monoidal categories between $\mathcal{C}^{\mathbf{c}} G$ and the categorical centre of $\mathcal{C}^{\mathbf{c}} \tilde{\mathcal{B}}^{2}$. To do this we first prove a weak form of the adjunction between truncated induction and truncated restriction. The adjunction is proved in full only as a consequence of Theorem 6.13. Another consequence of Theorem 6.13 is that the character sheaves of $G$ associated to $\mathbf{c}$ are in bijection with the simple objects of the categorical centre of $\mathcal{C}^{\mathbf{c}} \tilde{\mathcal{B}}^{2}$.

\section{Contents}

1. Study of the algebra $\mathbf{H}$.

2. Truncated convolution of sheaves on $\tilde{\mathcal{B}}^{2}$.

3. Sheaves on the variety $Z$.

4. The monoidal category $\mathcal{C}^{\mathbf{c}} \tilde{\mathcal{B}}^{2}$ and its centre.

5. Truncated induction, truncated restriction, truncated convolution on $G$.

6. The main results.

\section{Study of the Algebra $H$}

1.1. Let $N \mathbf{T}$ be the normalizer of $\mathbf{T}$ in $G$, let $W=N \mathbf{T} / \mathbf{T}$ be the Weyl group and let $\kappa: N \mathbf{T} \rightarrow W$ be the obvious homomorphism. For $w \in W$ we set $G_{w}=\mathbf{B} \kappa^{-1}(w) \mathbf{B}$ so that $G=\sqcup_{w} G_{w}$; let $\mathcal{O}_{w}=\left\{\left(x \mathbf{B} x^{-1}, y \mathbf{B} y^{-1}\right) ; x \in\right.$ $\left.G, y \in G, x^{-1} y \in G_{w}\right\}$ so that $\mathcal{B} \times \mathcal{B}=\sqcup_{w} \mathcal{O}_{w}$. For $w \in W$ let $\bar{G}_{w}$ be the closure of $G_{w}$ in $G$; we have $\bar{G}_{w}=\cup_{y \leq w} G_{y}$ for a well defined partial order $\leq$ on $W$. Let $\overline{\mathcal{O}}_{w}$ be the closure of $\mathcal{O}_{w}$ in $\mathcal{B}^{2}$. Now $W$ is a (finite) Coxeter group with length function $w \mapsto|w|=\operatorname{dim} \mathcal{O}_{w}-\nu$ and with set of generators $S=\{s \in W ;|s|=1\}$. It acts on $\mathbf{T}$ by $w: t \mapsto w(t)=\omega t \omega^{-1}$ where $\omega \in \kappa^{-1}(w)$. 
1.2. Let $s \in S$. Let $\mathbf{U}_{s}$ be the unique root subgroup of $\mathbf{U}$ with respect to $\mathbf{T}$ such that $\mathbf{U}_{s}^{-}:=\sigma \mathbf{U}_{s} \sigma^{-1} \not \subset \mathbf{U}$ for some/any $\sigma \in \kappa^{-1}(s)$. For any $\xi \in$ $\mathbf{U}_{s}-\{1\}$ there is a unique $\eta \in \mathbf{U}_{s}^{-}-\{1\}$ such that $\xi \eta \xi=\eta \xi \eta \in \kappa^{-1}(s) \subset N \mathbf{T}$; we set $\sigma_{\xi}=\xi \eta \xi=\eta \xi \eta$. We have $\sigma_{\xi}^{4}=1$. Note that $\xi \mapsto \eta$ is an isomorphism of algebraic varieties $\mathbf{U}_{s}-\{1\} \stackrel{\sim}{\rightarrow} \mathbf{U}_{s}^{-}-\{1\}$.

1.3. Following Tits we define a cross-section $W \rightarrow N \mathbf{T}, w \mapsto \dot{w}$ of $\kappa$ : $N \mathbf{T} \rightarrow W$ as follows. For each $s \in S$ we choose $\xi_{s} \in \mathbf{U}_{s}-\{1\}$. Let $w \in W$. We write $w=s_{1} s_{2} \ldots s_{r}$ where $s_{i} \in S, r=|w|$ and we set $\dot{w}=\sigma_{\xi_{s_{1}}} \sigma_{\xi_{s_{1}}} \sigma_{\xi_{s_{2}}} \ldots \sigma_{\xi_{s_{r}}} \in \kappa^{-1}(w)$. It is known that $\dot{w}$ is independent of the choice of $s_{1}, s_{2}, \ldots, s_{r}$. Clearly, if $w, w^{\prime} \in W$ satisfy $\left|w w^{\prime}\right|=|w|+\left|w^{\prime}\right|$, then $\left(w w^{\prime}\right)^{\circ}=\dot{w} \dot{w}^{\prime}$.

1.4. In this paper we fix an integer $n \geq 1$ such that $n \neq 0$ in $\mathbf{k}$. Let $\mathbf{T}_{n}=\left\{t \in \mathbf{T} ; t^{n}=1\right\}, \mathfrak{s}=\operatorname{Hom}\left(\mathbf{T}_{n}, \overline{\mathbf{Q}}_{l}^{*}\right)$. We have $\sharp\left(\mathbf{T}_{n}\right)=\sharp(\mathfrak{s})=n^{\rho}$. Define $\iota: \mathbf{T} \rightarrow \mathbf{T}$ by $t \mapsto t^{n}$; clearly, $\iota \overline{\mathbf{Q}}_{l}$ is a local system on $\mathbf{T}$, equivariant for the $\mathbf{T}$-action $t_{1}: t \mapsto t_{1}^{n} t$ on $\mathbf{T}$, hence $\mathbf{T}_{n}$ acts naturally on each stalk of $\iota_{!} \overline{\mathbf{Q}}_{l}$. We have $\iota_{!} \overline{\mathbf{Q}}_{l}=\oplus_{\lambda \in \mathfrak{s}} L_{\lambda}$, where for any $\lambda \in \mathfrak{s}, L_{\lambda}$ (a local system of rank 1 on $\mathbf{T}$ ) is such that $\mathbf{T}_{n}$ acts on each stalk of $L_{\lambda}$ through the character $\lambda$.

The $W$-action on $\mathbf{T}$ restricts to a $W$-action on $\mathbf{T}_{n}$ hence induces a $W$ action on $\mathfrak{s}$. We shall write $W \mathfrak{s}$ instead of $W \times \mathfrak{s}$ (without group structure); for $w \in W, \lambda \in \mathfrak{s}$ we shall write $w \cdot \lambda$ instead of $(w, \lambda)$. The following result can be deduced from [19, 28.2(a)].

(a) If $w \cdot \lambda \in W \mathfrak{s}$ and $w(\lambda)=\lambda$ then $L_{\lambda}$ is T-equivariant for the T-action $t: t^{\prime} \mapsto w(t)^{-1} t^{\prime} t$ on $T$.

1.5. Let $\tau \in \mathbf{T}$. We define $g_{\tau}: \mathbf{T} \rightarrow \mathbf{T}$ by $t \mapsto \tau t$. We show that for $\lambda \in \mathfrak{s}$, the local systems $g_{\tau}^{*} L_{\lambda}, L_{\lambda}$ are isomorphic. More precisely, we show that any $\tau^{\prime} \in \mathbf{T}$ such that $\tau^{\prime n}=\tau$ defines an isomorphism of local systems $g_{\tau}^{*} L_{\lambda} \stackrel{\sim}{\rightarrow} L_{\lambda}$. The induced map $\left(g_{\tau}^{*} L_{\lambda}\right)_{t}=\left(L_{\lambda}\right)_{\tau t} \stackrel{{ }^{\tau^{\prime}, t}}{\rightarrow}\left(L_{\lambda}\right)_{t}$ on stalks at any $t \in \mathbf{T}$ can be described as follows. We have

$$
\begin{aligned}
\left(L_{\lambda}\right)_{t} & =\left\{f: \iota^{-1}(t) \rightarrow \overline{\mathbf{Q}}_{l} ; f\left(t_{1} t^{\prime}\right)=\lambda\left(t^{\prime}\right) f\left(t_{1}\right) \forall t_{1} \in \iota^{-1}(t), t^{\prime} \in \mathbf{T}_{n}\right\} \\
\left(L_{\lambda}\right)_{\tau t} & =\left\{f^{\prime}: \iota^{-1}(\tau t) \rightarrow \overline{\mathbf{Q}}_{l} ; f^{\prime}\left(t_{2} t^{\prime}\right)=\lambda\left(t^{\prime}\right) f^{\prime}\left(t_{2}\right) \forall t_{2} \in \iota^{-1}(\tau t), t^{\prime} \in \mathbf{T}_{n}\right\}
\end{aligned}
$$


We have $c_{\tau^{\prime}, t}(f)=f^{\prime}$ where for any $t_{2} \in \iota^{-1}(\tau t)$ we have $f^{\prime}\left(t_{2}\right)=f\left(\tau^{\prime-1} t_{2}\right)$.

1.6. For any root $\alpha: \mathbf{T} \rightarrow \mathbf{k}^{*}$ we denote by $\check{\alpha}: \mathbf{k}^{*} \rightarrow \mathbf{T}$ the corresponding coroot and by $s_{\alpha}$ the corresponding reflection in $W$.

Let $\lambda \in \mathfrak{s}$. Let $R_{\lambda}$ be the set of roots $\alpha: T \rightarrow \mathbf{k}^{*}$ such that $\lambda(\check{\alpha}(z))=1$ for all $z \in \mathbf{k}^{*}, z^{n}=1$. Let $W_{\lambda}$ be the subgroup of $W$ generated by $\left\{s_{\alpha} ; \alpha \in\right.$ $\left.R_{\lambda}\right\}$. We have $W_{\lambda}=W_{\lambda^{-1}}$. Let $W_{\lambda}^{\prime}=\{w \in W ; w(\lambda)=\lambda\}$. Note that $W_{\lambda} \subset W_{\lambda}^{\prime}$. There is a unique Coxeter group structure on $W_{\lambda}$ with length function $W_{\lambda} \rightarrow \mathbf{N}, w \mapsto|w|_{\lambda}$ such that, if $w \in W_{\lambda}$ and $w=s_{1} s_{2} \ldots s_{r}$ is any reduced expression of $w$ in $W$, then

$$
|w|_{\lambda}=\operatorname{card}\left\{i \in[1, r] ; s_{r} \ldots s_{i+1} s_{i} s_{i+1} \ldots s_{r} \in W_{\lambda}\right\}
$$

See [14, 5.3].

1.7. As in [19, 31.2], let $\mathbf{H}_{n}$ be the associative $\mathcal{A}$-algebra with with generators $T_{w}(w \in W), 1_{\lambda}(\lambda \in \mathfrak{s})$ and relations:

$$
\begin{aligned}
1_{\lambda} 1_{\lambda^{\prime}} & =\delta_{\lambda, \lambda^{\prime}} 1_{\lambda} \text { for } \lambda, \lambda^{\prime} \in \mathfrak{s} ; \\
T_{w} T_{w^{\prime}} & =T_{w w^{\prime}} \text { if } w, w^{\prime} \in W \text { and }\left|w w^{\prime}\right|=|w|+\left|w^{\prime}\right| \\
T_{w} 1_{\lambda} & =1_{w(\lambda)} T_{w} \text { for } w \in W, \lambda \in \mathfrak{s} ; \\
T_{s}^{2} & =v^{2} T_{1}+\left(v^{2}-1\right) \sum_{\lambda ; s \in W_{\lambda}} T_{s} 1_{\lambda} \text { for } s \in W,|s|=1 ; \\
T_{1} & =\sum_{\lambda \in \mathfrak{s}} 1_{\lambda} .
\end{aligned}
$$

The algebra $\mathbf{H}_{n}$ is closely related to the algebra introduced by Yokonuma [30]. (It specializes to it under $v=\sqrt{q}, n=q-1$.) Since $n$ is fixed, we shall often write $\mathbf{H}$ instead of $\mathbf{H}_{n}$. Note that $T_{1}$ is the unit element of $\mathbf{H}$ and that $\left\{T_{w} 1_{\lambda} ; w \cdot \lambda \in W \mathfrak{s}\right\}$ is an $\mathcal{A}$-basis of $\mathbf{H}$. The $\mathcal{A}$-linear map ${ }^{\sim}: \mathbf{H} \rightarrow \mathbf{H}$, $T_{w} 1_{\lambda} \mapsto T_{w} 1_{\lambda^{-1}}$ is an algebra automorphism. The $\mathcal{A}$-linear map $\mathbf{H} \rightarrow \mathbf{H}$, $h \mapsto h^{b}$, given by $T_{w} 1_{\lambda} \mapsto 1_{\lambda} T_{w^{-1}}$ is an algebra antiautomorphism. (See [20, 32.19].)

1.8. For $w \in W$ we set $\hat{T}_{w}=v^{-|w|} T_{w} \in \mathbf{H}$. There is a unique ring homomorphism $^{-}: \mathbf{H} \rightarrow \mathbf{H}$ such that $\overline{f \hat{T}_{w} 1_{\lambda}}=\bar{f} \hat{T}_{w^{-1}}^{-1} 1_{\lambda}$ for any $w \cdot \lambda \in W \mathfrak{s}, f \in \mathcal{A}$; 
it has square 1. As in [20,34.4], for any $w \cdot \lambda \in W \mathfrak{s}$ there is a unique element $c_{w \cdot \lambda} \in \mathbf{H}$ such that

$$
c_{w \cdot \lambda}=\sum_{y \in W} p_{y \cdot \lambda, w \cdot \lambda} \hat{T}_{y} 1_{\lambda}
$$

where $p_{y \cdot \lambda, w \cdot \lambda} \in v^{-1} \mathbf{Z}\left[v^{-1}\right]$ if $y \neq w, p_{w \cdot \lambda, w \cdot \lambda}=1$ and $\overline{c_{w \cdot \lambda}}=c_{w \cdot \lambda}$. Since ${ }^{-}: \mathbf{H} \rightarrow \mathbf{H},{ }^{\sim}: \mathbf{H} \rightarrow \mathbf{H}$ commute, for any $w \cdot \lambda \in W \mathfrak{s}$, the element

$$
\widetilde{c_{w \cdot \lambda^{-1}}}=\sum_{y \in W} p_{y \cdot \lambda^{-1}, w \cdot \lambda^{-1}} \hat{T}_{y} 1_{\lambda}
$$

satisfies the definition of $c_{w \cdot \lambda}$ hence

$$
\widetilde{c_{w \cdot \lambda^{-1}}}=c_{w \cdot \lambda}
$$

In particular we have $p_{y \cdot \lambda^{-1}, w \cdot \lambda^{-1}}=p_{y \cdot \lambda^{-1}, w \cdot \lambda^{-1}}$ for any $y \cdot \lambda \in W \mathfrak{s}$.

For $y^{\prime}, w^{\prime}$ in $W_{\lambda}$ let $P_{y^{\prime}, w^{\prime}}^{\lambda}$ be the polynomial defined in [7] in terms of the Coxeter group $W_{\lambda}$; let

$$
p_{y^{\prime}, w^{\prime}}^{\lambda}=v^{-\left|w^{\prime}\right|_{\lambda}+\left|y^{\prime}\right|_{\lambda}} P_{y^{\prime}, w^{\prime}}^{\lambda}\left(v^{2}\right) \in \mathbf{Z}\left[v^{-1}\right] .
$$

Let $w \cdot \lambda \in W \mathfrak{s}$. From $[13,1.9(\mathrm{i})]$ we see that $w W_{\lambda}$ contains a unique element $z$ such that $|z|$ is minimum; we write $z=\min \left(w W_{\lambda}\right)$; we have $w=z w^{\prime}$ with $w^{\prime} \in W_{\lambda}$. We show:

$$
c_{z w^{\prime} \cdot \lambda}=\sum_{y^{\prime} \in W_{\lambda}} p_{y^{\prime}, w^{\prime}}^{\lambda} \hat{T}_{z y^{\prime}} 1_{\lambda} .
$$

Since $p_{y^{\prime}, w^{\prime}}^{\lambda}$ is 1 if $y^{\prime}=w^{\prime}$ and is in $v^{-1} \mathbf{Z}\left[v^{-1}\right]$ if $y^{\prime} \neq w^{\prime}$, it is enough to show that

$$
\sum_{y^{\prime} \in W_{\lambda}} p_{y^{\prime}, w^{\prime}}^{\lambda} \hat{T}_{z y^{\prime}} 1_{\lambda} \text { is fixed by }{ }^{-}: \mathbf{H} \rightarrow \mathbf{H} .
$$

We can find a sequence $s_{1}, s_{2}, \ldots, s_{k}$ in $S$ such that

$$
z(\lambda)=s_{1} s_{2} \ldots s_{k} \lambda \neq s_{2} \ldots s_{k} \lambda \neq \cdots \neq s_{k} \lambda \neq \lambda
$$

We argue by induction on $k$. If $k=0$ we have $z(\lambda)=\lambda$ and (b) follows from the proof of $[20,34.7]$. Assume now that $k \geq 1$. We have $z(\lambda) \neq\left(s_{1} z\right)(\lambda)$ 
hence $z^{-1} s_{1} z \lambda \neq \lambda$. This implies that $s_{1} z=\min \left(s_{1} z W_{\lambda}\right)$. We have $s_{1} z \lambda=$ $s_{2} \ldots s_{k} \lambda \neq \cdots \neq s_{k} \lambda \neq \lambda$; hence by the induction hypothesis applied to $s_{1} z$ instead of $z$ we see that

$$
\sum_{y^{\prime} \in W_{\lambda}} p_{y^{\prime}, w^{\prime}}^{\lambda}\left(v^{2}\right) \hat{T}_{s_{1} z y^{\prime}} 1_{\lambda} \text { is fixed by }{ }^{-}: \mathbf{H} \rightarrow \mathbf{H} .
$$

For $y^{\prime} \in W_{\lambda}$ we have $\hat{T}_{z y^{\prime}} 1_{\lambda}=\hat{T}_{s_{1}} 1_{\left(s_{1} z\right)(\lambda)} \hat{T} s_{1} z y^{\prime} 1_{\lambda}$ (we use again that $z(\lambda) \neq$ $\left.\left(s_{1} z\right)(\lambda)\right)$ and $\hat{T}_{s_{1}} 1_{\left(s_{1} z\right)(\lambda)}$ is fixed by ${ }^{-}: \mathbf{H} \rightarrow \mathbf{H}$ (using that $\left.z(\lambda) \neq\left(s_{1} z\right)(\lambda)\right)$; we see that (b) follows from (c) by left multiplication with $\hat{T}_{s_{1}} 1_{\left(s_{1} z\right)(\lambda)}$. This completes the proof of (a).

From (a) we see that

$$
p_{y \cdot \lambda, z w^{\prime} \cdot \lambda}= \begin{cases}p_{y^{\prime}, w^{\prime}}^{\lambda}\left(v^{2}\right) & \text { if } y=z y^{\prime}, y^{\prime} \in W_{\lambda}, \\ 0 & \text { if } y \notin z W_{\lambda} .\end{cases}
$$

In particular we have $p_{y \cdot \lambda, w \cdot \lambda} \in \mathbf{N}\left[v^{-1}\right]$. We show:

$$
1_{w(\lambda)} c_{w \cdot \lambda}=c_{w \cdot \lambda}
$$

Using (a) it is enough to show that $1_{w(\lambda)} \hat{T}_{z y^{\prime}} 1_{\lambda}=\hat{T}_{z y^{\prime}} 1_{\lambda}$ for any $y^{\prime} \in W_{\lambda}$. We have $\hat{T}_{z y^{\prime}} 1_{\lambda}=1_{\left(z y^{\prime}\right)(\lambda)} \hat{T}_{z y^{\prime}}$ and it is enough to show that $\left(z y^{\prime}\right)(\lambda)=\left(z w^{\prime}\right)(\lambda)$; this follows from $y^{\prime}(\lambda)=\lambda, w^{\prime}(\lambda)=\lambda$.

Let $w \cdot \lambda \in W \mathfrak{s}$. By (a) we have

$$
c_{w \cdot \lambda}=\sum_{y \in w W_{\lambda}} p_{y \cdot \lambda, w \cdot \lambda} \hat{T}_{y} 1_{\lambda}
$$

Similarly, we have

$$
c_{w^{-1} \cdot w(\lambda)}=\sum_{y \in w^{-1} W_{w(\lambda)}} p_{y \cdot w(\lambda), w^{-1} \cdot w(\lambda)} \hat{T}_{y} 1_{w(\lambda)} .
$$

It follows that

$$
\begin{aligned}
\left(c_{w^{-1} \cdot w(\lambda)}\right)^{b} & =\sum_{y \in w^{-1} W_{w(\lambda)}} p_{y \cdot w(\lambda), w^{-1} \cdot w(\lambda)} 1_{w(\lambda)} \hat{T}_{y^{-1}} \\
& =\sum_{y \in w^{-1} W_{w(\lambda)}} p_{y \cdot w(\lambda), w^{-1} \cdot w(\lambda)} \hat{T}_{y^{-1}} 1_{y w(\lambda)} .
\end{aligned}
$$


For each $y$ in the last sum we have $y=w^{-1} u$ with $u w(\lambda)=w(\lambda)$ hence $y w(\lambda)=w^{-1} u w(\lambda)=w^{-1} w(\lambda)=\lambda$. Thus, we have

$$
\begin{aligned}
\left(c_{w^{-1} \cdot w(\lambda)}\right)^{b} & =\sum_{y \in w^{-1} W_{w(\lambda)}} p_{y \cdot w(\lambda), w^{-1} \cdot w(\lambda)} \hat{T}_{y^{-1}} 1_{\lambda} \\
& =\sum_{y ; y^{-1} \in w^{-1} W_{w(\lambda)}} p_{y^{-1} \cdot w(\lambda), w^{-1} \cdot w(\lambda)} \hat{T}_{y} 1_{\lambda} .
\end{aligned}
$$

The condition that $y^{-1} \in w^{-1} W_{w(\lambda)}$ is equivalent to $y \in W_{w(\lambda)} w$ and also to $y \in w W_{\lambda}$. Hence

$$
\left(c_{w^{-1} \cdot w(\lambda)}\right)^{b}=\sum_{y ; y \in w W_{\lambda}} p_{y^{-1} \cdot w(\lambda), w^{-1} \cdot w(\lambda)} \hat{T}_{y} 1_{\lambda} .
$$

Note that $p_{y^{-1} \cdot w(\lambda), w^{-1} \cdot w(\lambda)}$ is 1 if $y=w$ and is in $v^{-1} \mathbf{Z}\left[v^{-1}\right]$ if $y \neq w$. Also, since $^{-}: \mathbf{H} \rightarrow \mathbf{H},{ }^{b}: \mathbf{H} \rightarrow \mathbf{H}$ commute, $\left(c_{w^{-1} \cdot w^{-1}(\lambda)}\right)^{b}$ is fixed by ${ }^{-}: \mathbf{H} \rightarrow \mathbf{H}$. It follows that $\left(c_{w^{-1} \cdot w(\lambda)}\right)^{b}$ satisfies the defining property of $c_{w \cdot \lambda}$, hence

$$
\left(c_{w^{-1} \cdot w(\lambda)}\right)^{b}=c_{w \cdot \lambda} \cdot
$$

We see also that

$$
p_{y^{-1} \cdot w(\lambda), w^{-1} \cdot w(\lambda)}=p_{y \cdot \lambda, w \cdot \lambda}
$$

for any $y \in W$.

1.9. Let $\mathfrak{A}$ be a based $\mathcal{A}$-algebra that is, an associative $\mathcal{A}$-algebra with 1 with a given finite basis $\left\{b_{i} ; i \in I\right\}$ as an $\mathcal{A}$-module, a given involution $i \mapsto i^{\text {! }}$ of $I$ such that the $\mathcal{A}$-linear map $x \mapsto x^{b}$ defined by $b_{i}^{b}=b_{i}$ ! for all $i \in I$ is an algebra antiautomorphism (necessarily preserving 1 ) and a given subset $I_{0}$ of $\left\{i \in I ; i^{!}=i\right\}$. For $i, i^{\prime}$ in $I$ we write $b_{i} b_{i^{\prime}}=\sum_{j \in I} h_{i, i^{\prime}, j} b_{j}$ where $h_{i, i^{\prime}, j} \in \mathcal{A}$. Let $j \preceq i$ (resp. $j \preceq i$ ) be the preorder on $I$ generated by the relation $h_{i^{\prime}, i, j} \neq 0$ for some $i^{\prime} \in I$, resp. by the relation

$$
h_{i, i^{\prime}, j} \neq 0 \text { or } h_{i^{\prime}, i, j} \neq 0 \text { for some } i^{\prime} \in I \text {. }
$$

We say that $i \sim j$ left $($ resp. $i \sim j$ ) if $i \preceq j$ and $j \preceq i$ left $($ resp. $i \preceq j$ and $j \preceq i$ ). This is an equivalence relation on $I$; the equivalence classes are called left cells (resp. two-sided cells). Note that any two-sided cell is a union of left cells. 
If $\mathbf{c}$ is a two-sided cell and $i \in I$ we write $i \preceq \mathbf{c}$ (resp. $\mathbf{c} \preceq i$ ) if $i \preceq i^{\prime}$ (resp. $i^{\prime} \preceq i$ ) for some $i^{\prime} \in \mathbf{c}$; we write $i \prec \mathbf{c}$ (resp. $\mathbf{c} \prec i$ ) if $i \preceq \mathbf{c}$ (resp. $\mathbf{c} \preceq i$ ) and $i \notin \mathbf{c}$. If $\mathbf{c}, \mathbf{c}^{\prime}$ are two-sided cells, we write $\mathbf{c} \preceq \mathbf{c}^{\prime}$ (resp. $\mathbf{c} \prec \mathbf{c}^{\prime}$ ) if $i \preceq i^{\prime}\left(\right.$ resp. $i \preceq i^{\prime}$ and $i \nsim i^{\prime}$ ) for some $i \in \mathbf{c}, i^{\prime} \in \mathbf{c}^{\prime}$.

Let $j \in I$. We can find an integer $m \geq 0$ such that $h_{i, i^{\prime}, j} \in v^{-m} \mathbf{Z}[v]$ for all $i, i^{\prime}$; let $a(j)$ be the smallest such $m$. For $i, i^{\prime}, j$ in $I$ there is a well defined integer $h_{i, i^{\prime}, j}^{*}$ such that

$$
h_{i, i^{\prime}, j^{!}}=h_{i, i^{\prime}, j}^{*} v^{-a\left(j^{!}\right)}+\text {higher powers of } v .
$$

We say that the based algebra $\mathfrak{A}$ is excellent if properties Q1-Q11 below hold.

Q1. If $j \in I_{0}$ and $i, i^{\prime} \in I$ satisfy $h_{i, i^{\prime}, j}^{*} \neq 0$ then $i^{\prime}=i^{*}$.

Q2. If $i \in I$, there exists a unique $j \in I_{0}$ such that $h_{i^{\prime}, i, j}^{*} \neq 0$.

Q3. If $i^{\prime} \preceq i$ then $a\left(i^{\prime}\right) \geq a(i)$. Hence if $i^{\prime} \sim i$ then $a\left(i^{\prime}\right)=a(i)$.

Q4. If $j \in I_{0}, i \in I$ and $h_{i^{!}, i, j}^{*} \neq 0$ then $h_{i^{\prime}, i, j}^{*}=1$.

Q5. For any $i, j, k$ in $I$ we have $h_{i, j, k}^{*}=h_{j, k, i}^{*}$.

Q6. Let $i, j, k$ in $I$ be such that $h_{i, j, k}^{*} \neq 0$. Then $i \underset{\text { left }}{\sim} j^{!}, j \sim k_{\text {left }}^{!}, k \sim i_{\text {left }}$.

Q7. If $i^{\prime} \preceq i$ and $a\left(i^{\prime}\right)=a(i)$ then $i^{\prime} \sim i$ left

Q8. If $i^{\prime} \preceq i$ and $a\left(i^{\prime}\right)=a(i)$ then $i^{\prime} \sim i$.

Q9. Any left cell $\Gamma$ of $I$ contains a unique element of $j \in I_{0}$. We have $h_{i^{\prime}, i, j}^{*}=1$ for all $i \in \Gamma$.

Q10. For any $i \in I$ we have $i \sim i^{!}$.

Q11. Let $v^{\prime}$ be a second indeterminate and let $h_{i, j, k}^{\prime} \in \mathbf{Z}\left[v^{\prime}, v^{\prime-1}\right]$ be obtained from $h_{i, j, k}$ by the substitution $v \mapsto v^{\prime}$, If $i, i^{\prime}, j, k \in I$ satisfy $a(j)=a(k)$ then

$$
\sum_{j^{\prime} \in I} h_{k, i^{\prime}, j^{\prime}}^{\prime} h_{i, j^{\prime}, j}=\sum_{j^{\prime} \in I} h_{i, k, j^{\prime}} h_{j^{\prime}, i^{\prime}, j}^{\prime}
$$

In the remainder of this subsection we assume that $\mathfrak{A}$ is excellent. Consider the free abelian group $\mathfrak{A}^{\infty}$ with basis $\left\{t_{i} ; i \in I\right\}$. We define a Z-bilinear multiplication $\mathfrak{A}^{\infty} \times \mathfrak{A}^{\infty} \rightarrow \mathfrak{A}^{\infty}$ by $t_{i} t_{i^{\prime}}=\sum_{j \in I} h_{i, i^{\prime}, j^{\prime}}^{*} t_{j}$. As in [18, 18.3], we see using Q3,Q6 that this defines an associative ring structure on $\mathfrak{A}^{\infty}$ and we see using Q1,Q2,Q4,Q5 that $\sum_{i \in I_{0}} t_{i}$ is 
a unit element for this ring structure. Also from Q1,Q5 we see that $t_{i} t_{i^{\prime}}=\delta_{i, i^{\prime}} t_{i}$ for $i, i^{\prime} \in I_{0}$.

From the definitions we have $h_{i^{!}, i^{\prime}, j !}=h_{i^{\prime}, i, j}$ for any $i, i^{\prime}, j$ in $I$. It follows that $a(j)=a\left(j^{!}\right)$for any $j$ (this also follows from Q3, Q10) and that $h_{i^{!}, i^{\prime}, j}^{*}=h_{i^{\prime}, i, j !}^{*}$ for any $i, i^{\prime}, j$ in $I$. Hence the Z-linear map ${ }^{b}: \mathfrak{A}^{\infty} \rightarrow \mathfrak{A}^{\infty}$ defined by $t_{i}^{b}=t_{i}$ ! for all $i \in I$ is a ring antiautomorphism.

We define an $\mathcal{A}$-linear map $\psi: \mathfrak{A} \rightarrow \mathcal{A} \otimes \mathfrak{A}^{\infty}$ by

$$
\psi\left(b_{i}\right)=\sum_{i^{\prime} \in I, j \in I_{0} ; a\left(i^{\prime}\right)=a(j)} h_{i, j, i^{\prime}} t_{i^{\prime}} .
$$

Using Q1,Q2,Q3,Q4,Q6,Q11 we see as in [18, 18.9] that $\psi$ is an $\mathcal{A}$-algebra homomorphism preserving 1 .

We define a group homomorphism $\tau: \mathfrak{A}^{\infty} \rightarrow \mathbf{Z}$ by $\tau\left(t_{i}\right)=1$ if $i \in I_{0}$, $\tau\left(t_{i}\right)=0$ if $i \in I-I_{0}$. We show:

(a) For $i, j \in I$ we have $\tau\left(t_{i} t_{j}\right)=1$ if $j=i^{!}$and $\tau\left(t_{i} t_{j}\right)=0$ if $j \neq i^{!}$.

An equivalent statement is that $\sum_{k \in I_{0}} h_{i, j, k}^{*}$ is 1 if $j=i^{!}$and is 0 if $j \neq i^{!}$. This follows immediately from Q1,Q2,Q4.

For any two-sided cell $\mathbf{c}$ in $I$ let $\mathfrak{A}_{\mathbf{c}}^{\infty}=\sum_{i \in \mathbf{c}} \mathbf{Z} t_{i} \subset \mathfrak{A}^{\infty}$. From Q6 we see that if $\mathbf{c}, \mathbf{c}^{\prime}$ are two-sided cells then $\mathfrak{A}_{\mathbf{c}}^{\infty} \mathfrak{A}_{\mathbf{c}^{\prime}}^{\infty}$ is zero if $\mathbf{c} \neq \mathbf{c}^{\prime}$ and is contained in $\mathfrak{A}_{\mathbf{c}}^{\infty}$ if $\mathbf{c}=\mathbf{c}^{\prime}$. Hence $\mathfrak{A}_{\mathbf{c}}^{\infty}$ is a ring with unit $\sum_{i \in I_{0} \cap \mathbf{c}} t_{i}$ and $\mathfrak{A}^{\infty}=\oplus_{\mathbf{c}} \mathfrak{A}_{\mathbf{c}}^{\infty}$ as rings.

1.10. Let $\mathfrak{A}$ be a based $\mathcal{A}$-algebra with basis $\left\{b_{i} ; i \in I\right\}$ and with $i \mapsto i^{!}, I_{0}$ as in 1.9. We assume that $\mathfrak{A}$ is excellent. We use the notation in 1.9. We fix a two-sided cell $\mathbf{c}$ of $I$ and we set $a=a(i)$ for any $i \in \mathbf{c}$. Let $r \geq 1$, let $\left(i_{1}, i_{2}, \ldots, i_{r}\right) \in I^{r}$. We write

$$
b_{i_{1}} b_{i_{2}} \ldots b_{i_{r}}=\sum_{i \in I, k \in \mathbf{Z}} N(i, k) v^{k} b_{i} \text { where } N(i, k) \in \mathbf{Z} \text {. }
$$

We show:

(a) Assume that $i \in \mathbf{c}$. If $N(i, k) \neq 0$ then $k \geq-(r-1) a$. If $N(i,-(r-1) a) \neq$ 0 then $i_{u} \in \mathbf{c}$ for all $u \in[1, r]$. 
If $r=1$ the result is obvious. Now assume that $r \geq 2$. We have

$$
\sum_{k \in \mathbf{Z}} N(i, k) v^{k}=\sum h_{j_{1}, i_{2}, j_{2}} h_{j_{2}, i_{3}, j_{3}} \ldots h_{j_{r-1}, i_{r}, j_{r}}
$$

where the last sum is taken over all $j_{1}, j_{2}, \ldots, j_{r-1}, j_{r}$ in $I$ such that

$$
i=j_{r} \preceq j_{r-1} \preceq \cdots \preceq j_{3} \preceq j_{2} \preceq j_{1}=i_{1} \text {. }
$$

Assume that $N(i, k) \neq 0$. From (b) we see that

$$
\begin{array}{r}
k=k_{2}+k_{3}+\cdots+k_{r} \text { where } k_{2} \geq-a\left(j_{2}\right), \ldots, k_{r-1} \geq-a\left(j_{r-1}\right), \\
k_{r} \geq-a\left(j_{r}\right)=-a
\end{array}
$$

for some $j_{1}, j_{2}, \ldots, j_{r-1}, j_{r}$ as above. Using $Q 3$ we see that

$$
a=a\left(j_{r}\right) \geq a\left(j_{r-1}\right) \geq \cdots \geq a\left(j_{3}\right) \geq a\left(j_{2}\right),
$$

hence $k_{2} \geq-a, \ldots, k_{r} \geq-a$ and $k \geq-(r-1) a$, as required.

Assume now that $N(i,-(r-1) a) \neq 0$. Then for some $j_{1}, j_{2}, j_{3}, \ldots$, $j_{r-1}, j_{r}$ as above, the inequalities used above must be equalities

$$
-k_{2}=\cdots=-k_{r-1}=a=a\left(j_{r}\right)=\cdots=a\left(j_{3}\right)=a\left(j_{2}\right)
$$

and

$$
h_{i_{1}, i_{2}, j_{2}^{!}}^{*} \neq 0, h_{j_{2}, i_{3}, j_{3}^{!}}^{*} \neq 0, \ldots, h_{j_{r-1}, i_{r}, j_{r}^{!}}^{*} \neq 0,
$$

so that, by $Q 6, Q 8$ we have

$$
i_{1} \sim i_{2} \sim j_{2} \sim i_{3} \sim j_{3} \sim \ldots \sim j_{r-1} \sim i_{r} \sim j_{r} .
$$

Thus, $i_{1} \in \mathbf{c}, \ldots, i_{r} \in \mathbf{c}$. This proves (a).

We show:

(c) Assume that $i \in \mathbf{c}$ and $i_{1} \in \mathbf{c}, \ldots, i_{r} \in \mathbf{c}$. Then

$$
N(i,-(r-1) a)=\sum h_{j_{1}, i_{2}, j_{2}^{!}}^{*} h_{j_{2}, i_{3}, j_{3}^{!}}^{*} \ldots h_{j_{r-1}, i_{r}, j_{r}^{!}}^{*}
$$

where the sum is taken over all $j_{1}, j_{2}, j_{3}, \ldots, j_{r-1}, j_{r}$ in $\mathbf{c}$ such that $j_{1}=$ $i_{1}, j_{r}=i$. 
Note that $N(i,-(r-1) a)$ is the coefficient of $v^{-(r-1) a}$ in

$$
\sum h_{j_{1}, i_{2}, j_{2}} h_{j_{2}, i_{3}, j_{3}} \ldots h_{j_{r-1}, i_{r}, j_{r}}
$$

where sum is taken over all $j_{1}, j_{2}, \ldots, j_{r-1}, j_{r}$ in $I$ such that

$$
i=j_{r} \preceq j_{r-1} \preceq \cdots \preceq j_{3} \preceq j_{2} \preceq j_{1}=i_{1}
$$

Such $j_{u}$ must satisfy $j_{u} \in \mathbf{c}$ for all $u$ (since $j_{1} \in \mathbf{c}, j_{r} \in \mathbf{c}$ ). Hence the sum is equal to

$$
\begin{gathered}
\sum\left(h_{j_{1}, i_{2}, j_{2}^{!}}^{*} v^{-a}+\text { higher powers of } v\right)\left(h_{j_{2}, i_{3}, j_{3}^{!}}^{*} v^{-a}+\text { higher powers of } v\right) \ldots \\
\left(h_{j_{r-1}, i_{r}, j_{r}^{\prime}}^{*} v^{-a}+\text { higher powers of } v\right) \\
=\sum h_{j_{1}, i_{2}, j_{2}^{!}}^{*} h_{j_{2}, i_{3}, j_{3}^{!}}^{*} \ldots h_{j_{r-1}, i_{r}, j_{r}^{!}}^{*} v^{-(r-1) a}+\text { higher powers of } v
\end{gathered}
$$

where both sums are taken over all $j_{1}, j_{2}, \ldots, j_{r-1}, j_{r}$ in $\mathbf{c}$ such that $j_{1}=i_{1}$, $j_{r}=i$. Now (c) follows.

From (c) we deduce:

(d) Assume that $i_{1} \in \mathbf{c}, \ldots, i_{r} \in \mathbf{c}$. Then

$$
t_{i_{1}} t_{i_{2}} \ldots t_{i_{r}}=\sum_{i \in \mathbf{c}} N(i,-(r-1) a) t_{i}
$$

$\left(\right.$ in $\left.\mathfrak{A}^{\infty}\right)$ where $N(i,-(r-1) a)$ is as in $(\mathrm{c})$.

We show:

(e) Assume that $i_{u} \in \mathbf{c}$ for some $u \in[1, r]$ and that $i \in I, k \in \mathbf{Z}$ are such that $N(i, k) \neq 0$. Then either $i \in \mathbf{c}, k \geq-(r-1) a$, or $i \prec \mathbf{c}$.

If $r=1$, the result is obvious. We now assume that $r \geq 2$. We have

$$
\sum_{k^{\prime} \in \mathbf{Z}} N\left(i, k^{\prime}\right) v^{k^{\prime}}=\sum h_{j_{1}, i_{2}, j_{2}} h_{j_{2}, i_{3}, j_{3}} \ldots h_{j_{r-1}, i_{r}, j_{r}}
$$

where the last sum is taken over all $j_{1}, j_{2}, \ldots, j_{r}$ in $I$ such that $j_{1}=i_{1}, j_{r}=i$. Since the left hand side is $\neq 0$, so is the right hand side. Thus there exist 
$j_{1}, j_{2}, \ldots, j_{r}$ as above such that

$$
h_{j_{1}, i_{2}, j_{2}} \neq 0, h_{j_{2}, i_{3}, j_{3}} \neq 0, \ldots, h_{j_{r-1}, i_{r}, j_{r}} \neq 0
$$

hence $j_{r} \preceq j_{r-1} \preceq \cdots \preceq j_{2} \preceq j_{1}$ and $j_{u} \preceq i_{u}$. In particular we have $j_{r} \preceq i_{u}$ that is, $i \preceq \mathbf{c}$. If $i \prec \mathbf{c}$, there is nothing to prove. Thus we may assume that $i \in \mathbf{c}$. In this case we have $k \geq-(r-1) a$ by (a). This proves (e).

1.11. We now give some examples of excellent based $\mathcal{A}$-algebras.

(i) Let $\lambda \in \mathfrak{s}$. Let $\mathbf{H}_{\lambda}$ be the Hecke algebra of the Coxeter group $W_{\lambda}$ with its basis $\left\{c_{w} ; w \in I=W_{\lambda}\right\}$ defined as in [18, 5.3] with $i \mapsto i$ ! given by $w \mapsto w^{-1}$ and $I_{0}$ being the set of distinguished involutions of $W_{\lambda}$ (defined as the set $\mathcal{D}$ in $[18,14.1]$ with $W$ replaced by $W_{\lambda}$ ). Then $\mathbf{H}_{\lambda}$ is excellent by results in [18, $\S 14, \S 15]$.

(ii) Let $\lambda \in \mathfrak{s}$. As in [20, 34.2], $W_{\lambda}^{\prime}$ is a semidirect product $W_{\lambda} \Omega_{\lambda}$ where $\Omega_{\lambda}$ is an abelian subgroup of $W_{\lambda}^{\prime}$ such that any $x \in \Omega_{\lambda}$ satisfies $x W_{\lambda} x^{-1}=W_{\lambda}$ and $\left|x w x^{-1}\right|_{\lambda}=|w|_{\lambda}$ for any $w \in W_{\lambda}$. Let $\mathbf{H}_{\lambda}^{\prime}$ be the $\mathcal{A}$-module $\mathbf{H}_{\lambda} \otimes_{\mathcal{A}} \mathcal{A}\left[\Omega_{\lambda}\right]$ with basis $\left\{c_{w} \otimes x ; w \in W_{\lambda}, x \in \Omega_{\lambda}\right\}$. We regard $\mathbf{H}_{\lambda}^{\prime}$ as an $\mathcal{A}$-algebra with multiplication $\left(c_{w} \otimes x\right)\left(c_{w^{\prime}} \otimes x^{\prime}\right)=\left(c_{w} c_{x w^{\prime} x^{-1}}\right) \otimes\left(x x^{\prime}\right)$ for $w, w^{\prime}$ in $W_{\lambda}$ and $x, x^{\prime}$ in $\Omega_{\lambda}$. We take $I=W_{\lambda} \times \Omega_{\lambda}, i \mapsto i^{!}$given by $(w, x) \mapsto\left(x^{-1}\left(w^{-1}\right), x^{-1}\right)$ and $I_{0}$ to be the set of all $(d, 1)$ where $d$ is a distinguished involution of $W_{\lambda}$. Then $\mathbf{H}_{\lambda}^{\prime}$ is excellent. (This follows easily from Case (i)).

(iii) Let $\lambda \in \mathfrak{s}$. Let $\mathfrak{A}=1_{\lambda} \mathbf{H} 1_{\lambda}$ viewed as a subalgebra of $\mathbf{H}$ with unit element $1_{\lambda}$ and with the basis $\left\{c_{w \cdot \lambda} ; w \in W_{\lambda}^{\prime}\right\}$. In this case we take $I=W_{\lambda}^{\prime}$. The involution $i \mapsto i^{\text {! }}$ is given by $w \mapsto w^{-1}$ for $w \in W_{\lambda}^{\prime}$. This is induced by the antiautomorphism of $\mathfrak{A}$ which is the restriction of the antiautomorphism $h \mapsto h^{b}$ of $\mathbf{H}$. We take $I_{0}$ to be the set of distinguished involutions of the Coxeter group $W_{\lambda}$. In [18, 34.7] it is shown that $\mathfrak{A}$ is canonically isomorphic as a based $\mathcal{A}$-algebra to $\mathbf{H}_{\lambda}^{\prime}$ in (ii). It follows that $\mathfrak{A}$ is excellent.

(iv) Let $\mathfrak{o}$ be a fixed $W$-orbit on $\mathfrak{s}$ and let $\lambda_{0} \in \mathfrak{o}$. Let $\mathbf{E}$ be the set of all formal sums $x=\sum_{\lambda, \lambda^{\prime} \in \mathfrak{o}} x_{\lambda, \lambda^{\prime}}$ where $x_{\lambda, \lambda^{\prime}} \in 1_{\lambda_{0}} \mathbf{H} 1_{\lambda_{0}}$ regarded naturally as an $\mathcal{A}$-module and as an $\mathcal{A}$-algebra where the product $x y$ of $x, y \in \mathbf{E}$ 
is given by

$$
(x y)_{\lambda, \lambda^{\prime}}=\sum_{\tilde{\lambda} \in \mathfrak{o}} x_{\lambda, \tilde{\lambda}} y_{\tilde{\lambda}, \lambda^{\prime}}
$$

(we used the product in $\left.1_{\lambda_{0}} \mathbf{H} 1_{\lambda_{0}}\right)$. Let $I=\left\{\left(w, \lambda, \lambda^{\prime}\right) \in W_{\lambda_{0}}^{\prime} \times \mathfrak{o} \times \mathfrak{o}\right\}$. We view $\mathbf{E}$ as a based $\mathcal{A}$-algebra with basis $\left\{b_{w, \lambda, \lambda^{\prime}} ;\left(w, \lambda, \lambda^{\prime}\right) \in I\right\}$ where $b_{w, \lambda, \lambda^{\prime}}$ has $\left(\lambda, \lambda^{\prime}\right)$-coordinate $c_{w \cdot \lambda_{0}}$ and all other coordinates zero. The involution $i \mapsto i^{!}$of $I$ is given by $\left(w, \lambda, \lambda^{\prime}\right) \mapsto\left(w^{-1}, l^{\prime}, \lambda\right)$. This is induced by the antiautomorphism $h \mapsto h^{\diamond}$ of the algebra $\mathbf{E}$ such that $b_{w, \lambda, \lambda^{\prime}}^{\diamond}=b_{w^{-1}, \lambda^{\prime}, \lambda}$. The subset $I_{0}$ of $I$ is the set of all $\left(w, \lambda, \lambda^{\prime}\right) \in I$ such that $\lambda=\lambda^{\prime}$ and $w \in \mathcal{D}$, the set of distinguished involutions of $W_{\lambda_{0}}$. For $w, w^{\prime}$ in $W_{\lambda_{0}}^{\prime}$ we write

$$
c_{w \cdot \lambda_{0}} c_{w^{\prime} \cdot \lambda_{0}}=\sum_{w^{\prime \prime} \in W_{\lambda_{0}}^{\prime}} h_{w, w^{\prime}, w^{\prime \prime}} c_{w^{\prime \prime} \cdot \lambda_{0}}
$$

where $h_{w, w^{\prime}, w^{\prime \prime}} \in \mathcal{A}$. Then the coefficients $h_{i_{1}, i_{2}, i_{3}}$ for $i_{1}, i_{2}, i_{3}$ in $I$ are given by

$$
h_{w, \lambda_{1}, \lambda_{2}, w^{\prime}, \lambda_{1}^{\prime}, \lambda_{2}^{\prime}, w^{\prime \prime}, \lambda_{1}^{\prime \prime}, \lambda_{2}^{\prime \prime}}=\delta_{\lambda_{2}, \lambda_{1}^{\prime}} \delta_{\lambda_{1}, \lambda_{1}^{\prime \prime}} \delta_{\lambda_{2}^{\prime}, \lambda_{2}^{\prime \prime}} h_{w, w^{\prime}, w^{\prime \prime}}
$$

We see that the $a$-function on $I$ is given by $a\left(w, \lambda, \lambda^{\prime}\right)=a(w)$ where $a(w)$ is computed in $1_{\lambda_{0}} \mathbf{H} 1_{\lambda_{0}}$. Moreover,

$$
h_{w, \lambda_{1}, \lambda_{2}, w^{\prime}, \lambda_{1}^{\prime}, \lambda_{2}^{\prime}, w^{\prime \prime}, \lambda_{1}^{\prime \prime}, \lambda_{2}^{\prime \prime}}^{*}=\delta_{\lambda_{2}, \lambda_{1}^{\prime}} \delta_{\lambda_{2}^{\prime}, \lambda_{1}^{\prime \prime}} \delta_{\lambda_{2}^{\prime \prime}, \lambda_{1}} h_{w, w^{\prime}, w^{\prime \prime}}^{*}
$$

We show that Q1-Q11 hold for $\mathfrak{A}=\mathbf{E}$, using that we already know that they hold for $1_{\lambda_{0}} \mathbf{H} 1_{\lambda_{0}}$.

We prove Q1. Assume that $h_{w, \lambda_{1}, \lambda_{2}, w^{\prime}, \lambda_{1}^{\prime}, \lambda_{2}^{\prime}, w^{\prime \prime}, \lambda_{1}^{\prime \prime}, \lambda_{2}^{\prime \prime}} \neq 0$ where $w^{\prime \prime} \in \mathcal{D}$, $\lambda_{1}^{\prime \prime}=\lambda_{2}^{\prime \prime}$. We must have $\lambda_{2}=\lambda_{1}^{\prime}, \lambda_{1}=\lambda_{2}^{\prime \prime}=\lambda_{1}^{\prime \prime}=\lambda_{2}^{\prime}, w^{\prime}=w^{-1}$ hence $\left(w, \lambda_{1}, \lambda_{2}\right)^{!}=\left(w^{\prime}, \lambda_{1}^{\prime}, \lambda_{2}^{\prime}\right)$. Thus Q1 holds.

We prove Q2. Assume that $h_{w, \lambda_{1}, \lambda_{2}, w^{-1}, \lambda_{2}, \lambda_{1}, w^{\prime \prime}, \lambda_{1}^{\prime \prime}, \lambda_{1}^{\prime \prime}}^{*} \neq 0$ where $w^{\prime \prime} \in \mathcal{D}$. Then $l_{1}=\lambda_{1}^{\prime \prime}$ and $w^{\prime \prime}$ is the unique element of $\mathcal{D}$ such that $h_{w, w^{-1}, w^{\prime \prime}}^{*} \neq 0$; thus the uniqueness in Q2 holds. The same proof shows the existence in Q2.

We prove Q3. If $i=\left(w, \lambda_{1}, \lambda_{2}\right), i^{\prime}=\left(w^{\prime}, \lambda_{1}^{\prime}, \lambda_{2}^{\prime}\right)$ then we have $i \preceq i^{\prime}$ (resp. $i \sim i^{\prime}$ ) in $\mathbf{E}$ if and only if $w \preceq w^{\prime}$ (resp. $w \sim w^{\prime}$ ) in $1_{\lambda_{0}} \mathbf{H} 1_{\lambda_{0}}$. Hence Q3 for $\mathbf{E}$ follows from Q3 for $1_{\lambda_{0}} \mathbf{H} 1_{\lambda_{0}}$. 
We prove Q4. Assume that $h_{w, \lambda_{1}, \lambda_{2}, w^{-1}, \lambda_{2}, \lambda_{1}, w^{\prime \prime}, \lambda_{1}^{\prime \prime}, \lambda_{1}^{\prime \prime}}^{*} \neq 0$ where $w^{\prime \prime} \in \mathcal{D}$. Then $h_{w, w^{-1}, w^{\prime \prime}}^{*} \neq 0$ hence by Q4 for $1_{\lambda_{0}} \mathbf{H} 1_{\lambda_{0}}$ we have $h_{w, w^{-1}, w^{\prime \prime}}^{*}=1$. It follows that $h_{w, \lambda_{1}, \lambda_{2}, w^{-1}, \lambda_{2}, \lambda_{1}, w^{\prime \prime}, \lambda_{1}^{\prime \prime}, \lambda_{1}^{\prime \prime}}^{*}=1$ as required.

We prove Q5. We must show that

$$
\delta_{\lambda_{2}, \lambda_{1}^{\prime}} \delta_{\lambda_{2}^{\prime}, \lambda_{1}^{\prime \prime}} \delta_{\lambda_{2}^{\prime \prime}, \lambda_{1}} h_{w, w^{\prime}, w^{\prime \prime}}^{*}=\delta_{\lambda_{2}^{\prime}, \lambda_{1}^{\prime \prime}} \delta_{\lambda_{2}^{\prime \prime}, \lambda_{1}} \delta_{\lambda_{2}, \lambda_{1}^{\prime}} h_{w^{\prime}, w^{\prime \prime}, w}^{*}
$$

This clearly follows from Q5 for $1_{\lambda_{0}} \mathbf{H} 1_{\lambda_{0}}$.

We prove Q6. If $i=\left(w, \lambda_{1}, \lambda_{2}\right), i^{\prime}=\left(w^{\prime}, \lambda_{1}^{\prime}, \lambda_{2}^{\prime}\right)$ then we have $i \preceq i_{\text {left }}^{\prime}$ (resp. $i \underset{\text { left }}{\sim \sim i^{\prime}}$ ) in $\mathbf{E}$ if and only if $\lambda_{2}=\lambda_{2}^{\prime}$ and $w \underset{\text { left }}{\preceq} w^{\prime}$ (resp. $\left.w \underset{\text { left }}{\sim} w^{\prime}\right)$ in $1_{\lambda_{0}} \mathbf{H} 1_{\lambda_{0}}$. Assume that $h_{w, \lambda_{1}, \lambda_{2}, w^{\prime}, \lambda_{1}^{\prime}, \lambda_{2}^{\prime}, w^{\prime \prime}, \lambda_{1}^{\prime \prime}, \lambda_{2}^{\prime \prime}}^{*} \neq 0$. Then $\delta_{\lambda_{2}, \lambda_{1}^{\prime}} \delta_{\lambda_{2}^{\prime}, \lambda_{1}^{\prime \prime}} \delta_{\lambda_{2}^{\prime \prime}, \lambda_{1}} h_{w, w^{\prime}, w^{\prime \prime}}^{*} \neq 0$ hence $\lambda_{2}=\lambda_{1}^{\prime}, \lambda_{2}^{\prime}=\lambda_{1}^{\prime \prime}, \lambda_{2}^{\prime \prime}=\lambda_{1}$ and (by Q6 for $1_{\lambda_{0}} \mathbf{H} 1_{\lambda_{0}}$ ) we have $\underset{\text { left }}{\sim} w^{\prime-1}$, $w^{\prime} \underset{\text { left }}{\sim} w^{\prime \prime-1}, w_{\text {left }}^{\sim} w^{-1}$. Thus Q6 holds for $\mathbf{E}$.

We prove Q8. Assume that $i=\left(w, \lambda_{1}, \lambda_{2}\right), i^{\prime}=\left(w^{\prime}, \lambda_{1}^{\prime}, \lambda_{2}^{\prime}\right)$ and $i \preceq i^{\prime}$, $a(i)=a\left(i^{\prime}\right)$. Then $w \preceq w^{\prime}$ and $a(w)=a\left(w^{\prime}\right)$ in $1_{\lambda_{0}} \mathbf{H} 1_{\lambda_{0}}$ so that by Q8 for $1_{\lambda_{0}} \mathbf{H} 1_{\lambda_{0}}$ we have $w \sim w^{\prime}$ and $i \sim i^{\prime}$. This proves Q8. The proof of Q7 is entirely similar to that of Q8.

We prove Q9. Now $\Gamma$ is the set of all $\left(w, \lambda_{1}, \lambda_{2}\right)$ where $\lambda_{2}$ is fixed, $\lambda_{1}$ runs through $\mathfrak{o}$ and $w$ runs through a left cell $\Gamma_{0}$ of $W_{\lambda_{0}}^{\prime}$. Let $w$ be the unique element in $\mathcal{D} \cap \Gamma_{0}$. Then $\left(w, \lambda_{2}, \lambda_{2}\right)$ is the unique element of $I_{0} \cap \Gamma$. If $i=\left(w_{1}, \lambda_{1}, \lambda_{2}\right) \in \Gamma$ then $w_{1} \in \Gamma_{0}$ and

$$
h_{w_{1}^{-1}, \lambda_{2}, \lambda_{1}, w, \lambda_{1}, \lambda_{2}, w, \lambda_{2}, \lambda_{2}}^{*}=h_{w_{1}^{-1}, w_{1}, w}^{*}
$$

and this is 1 by Q9 for $1_{\lambda_{0}} \mathbf{H} 1_{\lambda_{0}}$.

We prove Q10. Let $\left(w, \lambda, \lambda^{\prime}\right) \in I$. It is enough to show that $w \sim w^{-1}$ in $1_{\lambda_{0}} \mathbf{H} 1_{\lambda_{0}}$; this follows from Q10 for $1_{\lambda_{0}} \mathbf{H} 1_{\lambda_{0}}$.

We prove Q11. We write $i=\left(w, \lambda_{1}, \lambda_{2}\right), i^{\prime}=\left(w^{\prime}, \lambda_{3}, \lambda_{4}\right), j=\left(u, \lambda_{5}, \lambda_{6}\right)$, $k=\left(z, \lambda_{7}, \lambda_{8}\right)$. We have $a(z)=a(u)$. We must show

$$
\begin{aligned}
& \delta_{\lambda_{8}, \lambda_{3}} \delta_{\lambda_{7}, \lambda_{2}} \delta_{\lambda_{1}, \lambda_{5}} \delta_{\lambda_{4}, \lambda_{6}} \sum_{u^{\prime}} h_{z, w^{\prime}, u^{\prime}}^{\prime} h_{w, u^{\prime}, u} \\
= & \delta_{\lambda_{8}, \lambda_{3}} \delta_{\lambda_{7}, \lambda_{2}} \delta_{\lambda_{1}, \lambda_{5}} \delta_{\lambda_{4}, \lambda_{6}} \sum_{u^{\prime}} h_{w, z, u^{\prime}} h_{u^{\prime}, w^{\prime}, u}^{\prime}
\end{aligned}
$$

This follows from Q11 for $1_{\lambda_{0}} \mathbf{H} 1_{\lambda_{0}}$.

We see that $\mathbf{E}$ is excellent. 
(v) Let $\mathfrak{o}$ be a fixed $W$-orbit on $\mathfrak{s}$. Let $\mathbf{H}_{\mathfrak{o}}$ be the $\mathcal{A}$-subalgebra of $\mathbf{H}$ with $\mathcal{A}$ basis $\left\{c_{w \cdot \lambda} ; w \in W, \lambda \in \mathfrak{o}\right\}$. We view $\mathbf{H}_{\mathfrak{o}}$ as a based $\mathcal{A}$-algebra. We take $I=\{w \cdot \lambda \in W \mathfrak{s} ; \lambda \in \mathfrak{o}\}$. The involution $i \mapsto i^{!}$of $I$ is $w \cdot \lambda \mapsto w^{-1} \cdot w(\lambda)$. This is induced by the antiautomorphism of $\mathbf{H}_{\mathfrak{o}}$ which is the restriction of the antiautomorphism $h \mapsto h^{b}$ of $\mathbf{H}$. We take $I_{0}$ to be the set of all $w \cdot \lambda$ where $\lambda \in \mathfrak{o}$ and $w$ is a distinguished involution of $W_{\lambda}$. We show that $\mathbf{H}_{\mathfrak{o}}$ is excellent. We fix $\lambda_{0} \in \mathfrak{o}$ and let $\mathbf{E}$ be as in (iv) above. For any $\lambda \in \mathfrak{o}$ we choose a sequence $\mathbf{s}_{\lambda}=\left(s_{1}, s_{2}, \ldots, s_{r}\right)$ in $S$ such that

$$
\lambda_{0} \neq s_{1} \lambda_{0} \neq s_{2} s_{1} \lambda_{0} \neq \cdots \neq s_{r} \ldots s_{2} s_{1}\left(\lambda_{0}\right)=\lambda
$$

and we set $\tau_{\lambda}=\hat{T}_{s_{1}} \hat{T}_{s_{2}} \ldots \hat{T}_{s_{r}} \in \mathbf{H},\left[\mathbf{s}_{\lambda}\right]=s_{1} s_{2} \ldots s_{r} \in W$. Note that $\tau_{\lambda}^{f}$ lat $=\hat{T}_{s_{r}} \ldots \hat{T}_{s_{2}} \hat{T}_{s_{1}} \in \mathbf{H}$. We define an $\mathcal{A}$-linear map $\Psi: \mathbf{H}_{\mathfrak{o}} \rightarrow \mathbf{E}$ by

$$
\Psi(h)_{\lambda_{1}, \lambda_{2}}=\tau_{\lambda_{1}} 1_{\lambda_{1}} h 1_{\lambda_{2}} \tau_{\lambda_{2}}^{b} \in 1_{\lambda_{0}} \mathbf{H} 1_{\lambda_{0}}
$$

for any $\lambda_{1}, \lambda_{2}$ in $\mathfrak{o}$. In [18, 34.10] it is shown that $\Psi$ is an isomorphism of $\mathcal{A}$-algebras and $\Psi^{-1}$ carries the basis element $b_{w, \lambda_{1}, \lambda_{2}}$ of $\mathbf{E}$ onto the basis element $c_{\left[\mathbf{s}_{\lambda_{1}}\right]^{-1} w\left[\mathbf{s}_{\lambda_{2}}\right] \cdot \lambda_{2}}$ of $\mathbf{H}_{\mathfrak{o}}$. We show that $\Psi\left(h^{b}\right)=(\Psi(h))^{\diamond}$ for all $h \in \mathbf{H}_{\mathfrak{o}}$. Indeed for $\lambda_{1}, \lambda_{2}$ in $\mathfrak{o}$ we have

$$
\begin{aligned}
\left((\Psi(h))^{\diamond}\right)_{\lambda_{1}, \lambda_{2}} & =\left(\Psi(h)_{\lambda_{2}, \lambda_{1}}\right)^{b}=\left(\tau_{\lambda_{2}} 1_{\lambda_{2}} h 1_{\lambda_{1}} \tau_{\lambda_{1}}^{b}\right)^{b} \\
& =\tau_{\lambda_{1}} 1_{\lambda_{1}} h^{b} 1_{\lambda_{2}} \tau_{\lambda_{2}}^{b}=\Psi\left(h^{b}\right)_{\lambda_{1}, \lambda_{2}} .
\end{aligned}
$$

If $w$ is a distinguished involution of $W_{\lambda_{0}}$ and $\lambda \in \mathfrak{o}$, then

$$
\Psi^{-1}\left(b_{w, \lambda, \lambda}\right)=c_{\left[\mathbf{s}_{\lambda}\right]^{-1} w\left[\mathbf{s}_{\lambda}\right] \cdot \lambda}
$$

note that conjugation by $\left[\mathbf{s}_{\lambda}\right]^{-1}$ is a Coxeter group isomorphism $W_{\lambda_{0}} \stackrel{\sim}{\rightarrow}$ $W_{\lambda}$ hence $\left[\mathbf{s}_{\lambda}\right]^{-1} w\left[\mathbf{s}_{\lambda}\right]$ is a distinguished involution of $W_{\lambda}$. This argument shows that $\Psi^{-1}$ induces a bijection from $I_{0}$ defined in terms of $\mathbf{E}$ to $I_{0}$ defined in terms of $\mathbf{H}_{\mathfrak{o}}$. Using the fact that $\mathbf{E}$ is excellent we now deduce that $\mathbf{H}_{\mathfrak{o}}$ is excellent.

(vi) We consider the $\mathcal{A}$-algebra $\mathbf{H}$ with its $\mathcal{A}$-basis $\left\{c_{w \cdot \lambda} ; w \cdot \lambda \in W \mathfrak{s}\right\}$. We view $\mathbf{H}$ as a based $\mathcal{A}$-algebra. We take $I=W \mathfrak{s}$. The involution $i \mapsto i^{\text {! }}$ of $I$ is $w \cdot \lambda \mapsto w^{-1} \cdot w(\lambda)$. This is induced by the antiautomorphism $h \mapsto h^{b}$ of $\mathbf{H}$. We take $I_{0}$ to be the set of all $w \cdot \lambda$ where $\lambda \in \mathfrak{s}$ and $w$ is a distinguished involution of $W_{\lambda}$. We have $\mathbf{H}=\oplus_{\mathfrak{o}} \mathbf{H}_{\mathfrak{o}}$ (as algebras) 
where $\mathfrak{o}$ runs over the set of $W$-orbits in $\mathfrak{s}$ and $\mathbf{H}_{\mathfrak{o}}$ is as in (v). Using the fact that each $\mathbf{H}_{\mathfrak{o}}$ is excellent, it follows immediately that $\mathbf{H}$ is excellent.

In this case we shall write $\mathbf{D}$ instead of $I_{0}$.

In particular, in case (vi) the two-sided cells of $W \mathfrak{s}$ and the $a$-function $a: W \mathfrak{s} \rightarrow \mathbf{N}$ are well defined. Note that each two-sided cell of $W \mathfrak{s}$ (in case (vi)) is equal to a two-sided cell of $W \times \mathfrak{o}$ (in case (v)) for a unique $W$-orbit $\mathfrak{o}$ in $\mathfrak{s}$. Moreover for any two-sided cell $\mathfrak{c}$ of $W \times \mathfrak{o}$ (with $\lambda \in \mathfrak{o}$ ), the subset $\left\{w \in W_{\lambda}^{\prime} ; w \cdot \lambda \in \mathfrak{c}\right\}$ is a two-sided cell of $W_{\lambda}^{\prime}$ (in case (ii)) and this gives a bijection between the set of two-sided cells of $W \times \mathfrak{o}$ and the set of two-sided cells of $W_{\lambda}^{\prime}$ in case (ii), which in turn is in bijection with the set of orbits of the conjugation action of $\Omega_{\lambda}$ on the set of two-sided cells of $W_{\lambda}$ in case (i).

The based algebras in (i) - (vi) have the additional properties that

(a) $h_{i, j, k} \in \mathbf{N}\left[v, v^{-1}\right], \overline{h_{i, j, k}}=h_{i, j, k}$ for any $i, j, k \in I$;

(b) $h_{i, j, k}^{*} \in \mathbf{N}$ for any $i, j, k \in I$.

Indeed, (a) is well known in the cases (i), (ii); from this we deduce by the arguments in (iii)-(vi) that (a) holds in each case (iii)-(vi). Clearly, (b) follows from (a). From (a) we see that for the based algebras in (iii)-(vi) we have

$$
h_{i, i^{\prime}, j^{!}}=h_{i, i^{\prime}, j}^{*} v^{a\left(j^{!}\right)}+\text {lower powers of } v \text {. }
$$

In (i) the ring $\mathbf{H}_{\lambda}^{\infty}$ has $\mathbf{Z}$-basis $\left\{t_{w} ; w \in W_{\lambda}\right\}$ in natural bijection with the $\mathcal{A}$-basis $\left(c_{w}\right)$ of $\mathbf{H}_{\lambda}$.

In (ii) the ring $\left(\mathbf{H}_{\lambda}^{\prime}\right)^{\infty}$ has $\mathbf{Z}$-basis $\left\{t_{w} \otimes x ; w \in W_{\lambda}, x \in \Omega_{\lambda}\right\}$ in natural bijection with the $\mathcal{A}$-basis $\left(c_{w} \otimes x\right)$ of $\mathbf{H}_{\lambda}^{\prime}$. The multiplication is given by

$$
\left(t_{w} \otimes x\right)\left(t_{w^{\prime}} \otimes x^{\prime}\right)=\sum_{z \in W_{\lambda}} c_{z} t_{z} \otimes\left(x x^{\prime}\right)
$$

where $t_{w} t_{x w^{\prime} x^{-1}}=\sum_{z \in W_{\lambda}} c_{z} t_{z}, c_{z} \in \mathbf{Z}$, is the product in $\mathbf{H}_{\lambda}^{\infty}$.

In (iii) we have an identification $\left(1_{\lambda} \mathbf{H} 1_{\lambda}\right)^{\infty}=\left(\mathbf{H}_{\lambda}^{\prime}\right)^{\infty}$ (as rings) for which the basis element $t_{(w x) \cdot \lambda}$ (with $w \in W_{\lambda}, x \in \Omega_{\lambda}$ ) of $\left(1_{\lambda} \mathbf{H} 1_{\lambda}\right)^{\infty}$ corresponds to the basis element $t_{w} \otimes x$ of $\left(\mathbf{H}_{\lambda}^{\prime}\right)^{\infty}$. 
In (iv) the ring $\mathbf{E}^{\infty}$ has $\mathbf{Z}$-basis $\left\{t_{w, \lambda, \lambda^{\prime}} ; w \in W_{\lambda_{0}}^{\prime}, \lambda \in \mathfrak{o}, \lambda^{\prime} \in \mathfrak{o}\right\}$ in natural bijection with the $\mathcal{A}$-basis $\left(b_{w, \lambda, \lambda^{\prime}}\right)$ of $\mathbf{E}$. This ring is canonically isomorphic to a matrix ring with entries in the ring $\left(1_{\lambda_{0}} \mathbf{H} 1_{\lambda_{0}}\right)^{\infty}$ with its natural basis.

In (v) we have an identification $\mathbf{H}_{\mathfrak{o}}^{\infty}=\mathbf{E}^{\infty}$ (as rings) for which the basis element $t_{w, \lambda_{1}, \lambda_{2}}$ of $\mathbf{E}^{\infty}$ corresponds to the basis element $t_{\left[\mathbf{s}_{\lambda_{1}}\right]^{-1} w\left[\mathbf{s}_{\lambda_{2}}\right] \cdot \lambda_{2}}$ of $\mathbf{H}_{\mathfrak{o}}^{\infty}$.

In (vi) we have an identification $\mathbf{H}^{\infty}=\oplus_{\mathfrak{o}} \mathbf{H}_{\mathfrak{o}}^{\infty}$ (as rings) for which the basis elements $t_{w \cdot \lambda}$ in the two sides correspond to each other.

1.12. For a based $\mathcal{A}$-algebra $\mathfrak{A}$ as in 1.9 we set $\mathfrak{A}^{v}=\overline{\mathbf{Q}}_{l}(v) \otimes_{\mathcal{A}} \mathfrak{A}$; we set $\mathfrak{A}^{1}=\overline{\mathbf{Q}}_{l} \otimes_{\mathcal{A}} \mathfrak{A}$ where $\overline{\mathbf{Q}}_{l}$ is viewed as an $\mathcal{A}$-algebra via $v \mapsto 1$. By definition, $\mathbf{H}^{1}$ is the associative $\overline{\mathbf{Q}}_{l}$-algebra with generators $T_{w}(w \in W), 1_{\lambda}(\lambda \in \mathfrak{s})$ and relations:

$$
\begin{aligned}
1_{\lambda} 1_{\lambda^{\prime}} & =\delta_{\lambda, \lambda^{\prime}} 1_{\lambda} & & \text { for } \lambda, \lambda^{\prime} \in \mathfrak{s} ; \\
T_{w} T_{w^{\prime}} & =T_{w w^{\prime}} & & \text { if } w, w^{\prime} \in W ; \\
T_{w} 1_{\lambda} & =1_{w(\lambda)} T_{w} & & \text { for } w \in W, \lambda \in \mathfrak{s} ; \\
T_{1} & =\sum_{\lambda \in \mathfrak{s}} 1_{\lambda} . & &
\end{aligned}
$$

The elements $\left\{T_{w} 1_{\lambda} ; w \cdot \lambda \in W \mathfrak{s}\right\}$ form an $\overline{\mathbf{Q}}_{l}$-basis of $\mathbf{H}^{1}$ and $T_{1}$ is the unit element. Consider the group algebra $\overline{\mathbf{Q}}_{l}\left[W \mathbf{T}_{n}\right]$ where $W \mathbf{T}_{n}$ is the semidirect product of $W$ and $\mathbf{T}_{n}$ with $\mathbf{T}_{n}$ normal and $W$ acting on $\mathbf{T}_{n}$ by $w: t \mapsto w(t)$. We define a $\overline{\mathbf{Q}}_{l}$-linear map $\overline{\mathbf{Q}}_{l}\left[W \mathbf{T}_{n}\right] \rightarrow \mathbf{H}^{1}$ by $w t \mapsto \sum_{\lambda \in \mathfrak{s}} \lambda(t) T_{w} 1_{\lambda}$. From the definitions we see that this is an isomorphism of $\overline{\mathbf{Q}}_{l}$-algebras; we shall use it to identify $\overline{\mathbf{Q}}_{l}\left[W \mathbf{T}_{n}\right]=\mathbf{H}^{1}$. For $\lambda \in \mathfrak{s}$ we set $\left(1_{\lambda} \mathbf{H} 1_{\lambda}\right)^{1}=\overline{\mathbf{Q}}_{l} \otimes_{\mathcal{A}}\left(1_{\lambda} \mathbf{H} 1_{\lambda}\right)$; under the identification above we have $\left(1_{\lambda} \mathbf{H} 1_{\lambda}\right)^{1}=\overline{\mathbf{Q}}_{l}\left[W_{\lambda}^{\prime}\right]$.

Recall that we have $\mathbf{H}^{\infty}=\oplus_{\mathbf{c}} \mathbf{H}_{\mathbf{c}}^{\infty}$ as rings. Here, for any two-sided cell $\mathbf{c}, \mathbf{H}_{\mathbf{c}}^{\infty}$ has basis $\left\{t_{w \cdot \lambda} ; w \cdot \lambda \in \mathbf{c}\right\}$; it is a ring with unit element $\sum_{w \cdot \lambda \in \mathbf{D}_{\mathbf{c}}} t_{w \cdot \lambda}$ where $\mathbf{D}_{\mathbf{c}}=\mathbf{D} \cap \mathbf{c}$. We set $\mathbf{J}=\overline{\mathbf{Q}}_{l} \otimes \mathbf{H}^{\infty}$. We have $\mathbf{J}=\oplus_{\mathbf{c}} \mathbf{J}_{\mathbf{c}}$ (as algebras) where for any two-sided cell $\mathbf{c}$ of $W \mathfrak{s}$ we set $\mathbf{J}_{\mathbf{c}}=\overline{\mathbf{Q}}_{l} \otimes \mathbf{H}_{\mathbf{c}}^{\infty}$.

Now $\psi: \mathbf{H} \rightarrow \mathcal{A} \otimes \mathbf{H}^{\infty}$ and $\psi: 1_{\lambda} \mathbf{H} 1_{\lambda} \rightarrow \mathcal{A} \otimes\left(1_{\lambda} \mathbf{H} 1_{\lambda}\right)^{\infty}$ induce by extension of scalars isomorphisms of split semisimple $\overline{\mathbf{Q}}_{l}(v)$-algebras $\psi^{v}$ : $\mathbf{H}^{v} \stackrel{\sim}{\rightarrow} \overline{\mathbf{Q}}_{l}(v) \otimes \mathbf{H}^{\infty}, \psi^{v}:\left(1_{\lambda} \mathbf{H} 1_{\lambda}\right)^{v} \stackrel{\sim}{\rightarrow} \overline{\mathbf{Q}}_{l}(v) \otimes\left(1_{\lambda} \mathbf{H} 1_{\lambda}\right)^{\infty}$ and isomorphisms 
of semisimple $\overline{\mathbf{Q}}_{l}$-algebras $\psi^{1}: \mathbf{H}^{1} \stackrel{\sim}{\rightarrow} \mathbf{J}, \psi^{1}: \overline{\mathbf{Q}}_{l}\left[W_{\lambda}^{\prime}\right] \stackrel{\sim}{\rightarrow} \overline{\mathbf{Q}}_{l} \otimes\left(1_{\lambda} \mathbf{H} 1_{\lambda}\right)^{\infty}$. (See $[20,34.12(\mathrm{~b}),(\mathrm{c})]$.

Let $\operatorname{Irr}\left(W \mathbf{T}_{n}\right)$ be a set of representatives for the isomorphism classes of simple $\overline{\mathbf{Q}}_{l}\left[W \mathbf{T}_{n}\right]=\mathbf{H}^{1}$-modules. For any $W \mathbf{T}_{n}$-module $E$ let $E^{\infty}$ be the corresponding $\mathbf{J}$-module (via $\psi^{1}$ ) and let $E^{v}$ be the $\mathbf{H}^{v}$-module corresponding to $\overline{\mathbf{Q}}_{l}(v) \otimes E^{\infty}$ under $\psi^{v}$.

For any $W$-orbit $\mathfrak{o}$ on $\mathfrak{s}$ let $\operatorname{Irr}_{\mathfrak{o}}\left(W \mathbf{T}_{n}\right)$ be the set of all $E \in \operatorname{Irr}\left(W \mathbf{T}_{n}\right)$ such that $1_{\lambda^{\prime}} E=0$ for all $\lambda^{\prime} \notin \mathfrak{o}$. We have $\operatorname{Irr}\left(W \mathbf{T}_{n}\right)=\sqcup_{\mathfrak{o}} \operatorname{Irr}_{\mathfrak{o}}\left(W \mathbf{T}_{n}\right)$. If $E \in \operatorname{Irr}_{\mathfrak{o}}\left(W \mathbf{T}_{n}\right)$, then for any $\lambda \in \mathfrak{o}, 1_{\lambda} E$ is a simple $\left(1_{\lambda} \mathbf{H} 1_{\lambda}\right)^{1}$-module, that is, a simple $W_{\lambda}^{\prime}$-module. Moreover $E \mapsto 1_{\lambda} E$ is a bijection between $\operatorname{Irr}_{\mathfrak{o}}\left(W \mathbf{T}_{n}\right)$ and a set of representatives $\operatorname{Irr}\left(W_{\lambda}^{\prime}\right)$ for the isomorphism classes of simple $\overline{\mathbf{Q}}_{l}\left[W_{\lambda}^{\prime}\right]$-modules.

For any $E \in \operatorname{Irr}_{\mathfrak{o}}\left(W \mathbf{T}_{n}\right)$ and $\lambda \in \mathfrak{o}$ let $\left(1_{\lambda} E\right)^{\infty}$ be the $\overline{\mathbf{Q}}_{l} \otimes\left(1_{\lambda} \mathbf{H} 1_{\lambda}\right)^{\infty}$ module corresponding to $1_{\lambda} E$ via $\psi^{1}$ and let $\left(1_{\lambda} E\right)^{v}$ be the $\left(1_{\lambda} \mathbf{H} 1_{\lambda}\right)^{v}$-module corresponding to $\overline{\mathbf{Q}}_{l}(v) \otimes\left(1_{\lambda} E\right)^{\infty}$ under $\psi^{v}$. Note that $\left(1_{\lambda} E\right)^{v}=1_{\lambda}\left(E^{v}\right)$.

If $E \in \operatorname{Irr}_{\mathfrak{o}}\left(W \mathbf{T}_{n}\right), \lambda \in \mathfrak{o}$ and $w \in W_{\lambda}^{\prime}$ then we have

$$
\operatorname{tr}\left(c_{w \cdot \lambda}, E^{v}\right)=\operatorname{tr}\left(c_{w \cdot \lambda},\left(1_{\lambda} E\right)^{v}\right) .
$$

For any left cell $\Lambda$ of $W \mathfrak{s}$ contained in o we denote by $[\Lambda]$ the $\overline{\mathbf{Q}}_{l}\left[W \mathbf{T}_{n}\right]$ module such that $[\Lambda]^{\infty}$ is the $\overline{\mathbf{Q}}_{l}$-subspace of $\mathbf{J}$ spanned by $\left\{t_{w \cdot \lambda} ; w \cdot \lambda \in \Lambda\right\}$ (a left ideal of $\mathbf{J}$ ). We show:

(a) Let $z \cdot \lambda$ be the unique element of $\Lambda \cap \mathbf{D}$. Then for any $E \in \operatorname{Irr}_{\mathfrak{o}} W \mathbf{T}_{n}$, $\operatorname{tr}\left(t_{z \cdot \lambda}, E^{\infty}\right)$ is equal to the multiplicity of $E^{\infty}$ in the $\mathbf{J}$-module $[\Lambda]^{\infty}$.

An equivalent statement is that

$$
\operatorname{dim}\left(t_{z \cdot \lambda} E^{\infty}\right)=\operatorname{dim} \operatorname{Hom}_{\mathbf{J}}\left([\Lambda]^{\infty}, E^{\infty}\right) .
$$

It is enough to show that the $\overline{\mathbf{Q}}_{l}$-linear map $\operatorname{Hom}_{\mathbf{J}}\left([\Lambda]^{\infty}, E^{\infty}\right) \rightarrow t_{z \cdot \lambda} E^{\infty}$, $\xi \mapsto \xi\left(t_{z \cdot \lambda}\right)$ is an isomorphism. The proof is along the same lines as that of $[18,21.3]$.

1.13. Let $\mathfrak{A}$ be one of the based $\mathcal{A}$-algebra $\mathbf{H}_{\lambda}, \mathbf{H}_{\lambda}^{\prime}($ with $\lambda \in \mathfrak{s})$ or $\mathbf{H}$. Note that in these cases $I$ is $W_{\lambda}, W_{\lambda}^{\prime}, W \mathfrak{s}$ respectively and $\mathfrak{A}^{1}$ is the group algebra $\overline{\mathbf{Q}}_{l}[\mathcal{W}]$ where $\mathcal{W}$ is $W_{\lambda}, W_{\lambda}^{\prime}, W \mathbf{T}_{n}$ respectively. Note that $\psi: \mathfrak{A} \rightarrow \mathcal{A} \otimes \mathfrak{A}^{\infty}$ 
induces an isomorphism $\overline{\mathbf{Q}}_{l}[\mathcal{W}]=\mathfrak{A}^{1} \stackrel{\sim}{\rightarrow} \overline{\mathbf{Q}}_{l} \otimes \mathfrak{A}^{\infty}$. Under this isomorphism an irreducible $\mathcal{W}$-module $E$ corresponds to a simple $\overline{\mathbf{Q}}_{l} \otimes \mathfrak{A}^{\infty}$-module $E^{\infty}$. We have $\overline{\mathbf{Q}}_{l} \otimes \mathfrak{A}^{\infty}=\oplus_{\mathbf{c}}\left(\overline{\mathbf{Q}}_{l} \otimes \mathfrak{A}_{\mathbf{c}}^{\infty}\right)$ where $\mathbf{c}$ runs over the two-sided cell of $I$. Hence if $E$ is an irreducible $\mathcal{W}$-module then there is a unique two-sided cell $\mathbf{c}_{E}$ of $I$ such that $\overline{\mathbf{Q}}_{l} \otimes \mathfrak{A}_{\mathbf{c}}^{\infty}$ acts as zero on $E^{\infty}$ for any $\mathbf{c} \neq \mathbf{c}_{E}$; thus $E^{\infty}$ can be viewed as a simple $\overline{\mathbf{Q}}_{l} \otimes \mathfrak{A}_{\mathbf{c}_{E}}^{\infty}$-module. For an irreducible $\mathcal{W}$-module $E$ let $a_{E} \in \mathbf{N}$ be the constant value of the restriction of $a: I \rightarrow \mathbf{N}$ to $\mathbf{c}_{E}$.

1.14. Since $\sim$ : $\mathbf{H} \rightarrow \mathbf{H}$ permutes the elements in the basis $\left\{c_{w \cdot \lambda}\right\}$ according to the involution $w \cdot \lambda \mapsto \widetilde{w \cdot \lambda}=w \cdot \lambda^{-1}$ of $W \mathfrak{s}$, we see that the image of a two-sided cell $\mathbf{c}$ of $W \mathfrak{s}$ under this involution is again a two-sided cell $\tilde{\mathbf{c}}$ of $W \mathfrak{s}$ and the value of the $a$-function on $\tilde{\mathbf{c}}$ is equal to the value of the $a$-function on $\mathbf{c}$.

1.15. Applying ${ }^{b}$ to the equation

we get

$$
c_{x \cdot \lambda} c_{y \cdot \lambda^{\prime}}=\sum_{z \cdot \lambda^{\prime \prime} \in W \mathfrak{s}} h_{x \cdot \lambda, y \cdot \lambda^{\prime}, z \cdot \lambda^{\prime \prime}} c_{z \cdot \lambda^{\prime \prime}}
$$

hence

$$
\begin{aligned}
c_{y^{-1} \cdot y\left(\lambda^{\prime}\right)} c_{x^{-1} \cdot x(\lambda)} & =\sum_{z \cdot \lambda^{\prime \prime} \in W \mathfrak{s}} h_{x \cdot \lambda, y \cdot \lambda^{\prime}, z \cdot \lambda^{\prime \prime}} c_{z^{-1} \cdot z\left(\lambda^{\prime \prime}\right)} \\
& =\sum_{z \cdot \lambda^{\prime \prime} \in W \mathfrak{s}} h_{x \cdot \lambda, y \cdot \lambda^{\prime}, z^{-1} \cdot z\left(\lambda^{\prime \prime}\right)} c_{z \cdot \lambda^{\prime \prime}}
\end{aligned}
$$

$$
h_{x \cdot \lambda, y \cdot \lambda^{\prime}, z^{-1} \cdot z\left(\lambda^{\prime \prime}\right)}=h_{y^{-1} \cdot y\left(\lambda^{\prime}\right), x^{-1} \cdot x(\lambda), z \cdot \lambda^{\prime \prime}}
$$

This shows that the involution $z \cdot \lambda \mapsto z^{-1} \cdot z(\lambda)$ of $W \mathfrak{s}$ preserves the preorder $\preceq$ hence it maps any two-sided cell onto a two-sided cell. (In fact, it maps each two-sided cell $\mathbf{c}$ onto itself. Indeed, it is enough to show that some element $z \cdot \lambda$ of $\mathbf{c}$ satisfies $z \cdot \lambda=z^{-1} \cdot z(\lambda)$; we can take $z \cdot \lambda$ to be any element of the nonempty subset $\mathbf{D}_{\mathbf{c}}$ of $\mathbf{c}$.) We also see that the $a$-function on $W \mathfrak{s}$ is constant on the orbits of our involution and that the group isomorphism ${ }^{b}: \mathbf{H}^{\infty} \rightarrow \mathbf{H}^{\infty}$ given by $t_{z \cdot \lambda} \mapsto t_{z^{-1} \cdot z(\lambda)}$ is a ring antiautomorphism. Note that our involution restricts to the identity permutation of the subset $\mathbf{D}$ of $W \mathfrak{s}$ and that the algebra homomorphism $\psi: \mathbf{H} \rightarrow \mathcal{A} \otimes \mathbf{H}^{\infty}$ is compatible with the antiautomorphisms ${ }^{b}$ of $\mathbf{H}$ and of $\mathcal{A} \otimes \mathbf{H}^{\infty}$. 
1.16. Let ()$^{\mathbf{Q}}: \overline{\mathbf{Q}}_{l} \rightarrow \overline{\mathbf{Q}}_{l}$ be a field automorphism which maps any root of 1 in $\overline{\mathbf{Q}}_{l}$ to its inverse. The field automorphism $\overline{\mathbf{Q}}_{l}(v) \rightarrow \overline{\mathbf{Q}}_{l}(v)$ which maps $v$ to $v$ and $x \in \overline{\mathbf{Q}}_{l}$ to $x^{\mathbf{\omega}}$ is denoted again by

1.17. We can view naturally $W$ as a subgroup of $G L(V)$ where

$$
V:=\overline{\mathbf{Q}}_{l} \otimes \operatorname{Hom}\left(\mathbf{T}, \mathbf{k}^{*}\right)(-1) .
$$

For any $i \geq 0$ let $S^{i} V$ be the $i$-th symmetric power of the vector space $V$. Then $S V=\oplus_{i \geq 0} S^{i} V$ is naturally a commutative algebra. Now $W$ acts naturally on $S^{i} V$ for any $i$.

Let $\lambda \in \mathfrak{s}$. If $E, \tilde{E} \in \operatorname{Mod} W_{\lambda}$ (resp. $\left.E^{\prime}, \tilde{E}^{\prime} \in \operatorname{Mod} W_{\lambda}^{\prime}\right)$ with $E$ (resp. $\left.E^{\prime}\right)$ irreducible, we set $\tilde{E}^{E}=\operatorname{Hom}_{W_{\lambda}}(E, \tilde{E})\left(\operatorname{resp} . \tilde{E}^{\prime E^{\prime}}=\operatorname{Hom}_{W_{\lambda}^{\prime}}\left(E^{\prime}, \tilde{E}^{\prime}\right)\right)$. For $E$ (resp. $E^{\prime}$ ) as above there exists $i \geq 0$ such that $\left(S^{i} V\right)^{E} \neq 0$ (resp. $\left(S^{i} V\right)^{E^{\prime}} \neq 0$ ); let $b_{E}$ (resp. $\left.b_{E^{\prime}}\right)$ be the smallest such $i$. If $E$ (resp. $E^{\prime}$ ) are as above we say that $E$ (resp. $\left.E^{\prime}\right)$ is univalent if $\operatorname{dim}\left(\left(S^{b_{E}} V\right)^{E}\right)=1$ (resp. $\left.\operatorname{dim}\left(\left(S^{b_{E^{\prime}}} V\right)^{E^{\prime}}\right)=1\right)$. We show:

(a) Let $E \in \operatorname{Mod} W_{\lambda}$ be irreducible and univalent. There exists $E^{\prime} \in \operatorname{Mod} W_{\lambda}^{\prime}$ irreducible such that $E$ appears in $\left.E^{\prime}\right|_{W_{\lambda}}$ and $b_{E^{\prime}}=b_{E}$; moreover, $E^{\prime}$ is uniquely determined up to isomorphism by these properties and is univalent.

Let $\underline{E}$ be the unique $W_{\lambda}$-submodule of $S^{b_{E}} V$ that is isomorphic to $E$. Let $E^{\prime}=\sum_{x \in \Omega_{\lambda}} x \underline{E} \subset S^{b_{E}} V$ (notation of 1.11(ii)) where we have used the $W$ action on $S^{b_{E}} V$. Then $E^{\prime}$ is a $W_{\lambda^{\prime}}^{\prime}$-submodule of $S^{b_{E}} V$; moreover, for each $x \in$

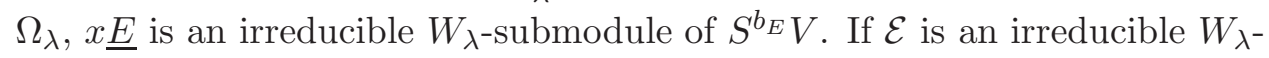
submodule of $E^{\prime}$ then $\mathcal{E}$ is isomorphic to $x \underline{E}$ as an irreducible $W_{\lambda}$-submodule (for some $x \in \Omega_{\lambda}$ ). But $x \underline{E}$ is a univalent $W_{\lambda}$-submodule hence we have necessarily $\mathcal{E}=x \underline{E}$. Since any irreducible $W_{\lambda}$-submodule of $E^{\prime}$ is equal to $x \underline{E}$ for some $x \in \Omega_{\lambda}$, we see that any nonzero $W_{\lambda}^{\prime}$-submodule of $E^{\prime}$ contains $x \underline{E}$ for some $x \in \Omega_{\lambda}$; being stable under the action of $\Omega_{\lambda}$, it is equal to $E^{\prime}$. Thus $E^{\prime}$ is an irreducible $W_{\lambda}^{\prime}$-submodule of $S^{b_{E}} V$. Clearly, $E^{\prime}$ appears with multiplicity 1 (resp. 0) in the $W_{\lambda}^{\prime}$-module $S^{b_{E}} V$ (resp. $S^{i} V$ with $0 \leq i<b_{E}$ ). Thus $b_{E^{\prime}}=b_{E}$ and $E^{\prime}$ is univalent. Thus the existence of $E^{\prime}$ in (a) is proved. Now let $E_{1}^{\prime}$ be an irreducible $W_{\lambda}^{\prime}$-module such that $E$ appears in $\left.E_{1}^{\prime}\right|_{W_{\lambda}}$ and $b E_{1}^{\prime}=b_{E}$. We can find a $W_{\lambda}^{\prime}$-submodule $\underline{E}_{1}^{\prime}$ of $S^{b_{E}} V$ that is isomorphic to $E_{1}^{\prime}$. By assumption we can find a $W_{\lambda}$-submodule of $\underline{E}_{1}^{\prime}$ which is isomorphic 
to $E$; this is necessarily equal to $\underline{E}$. For any $x \in \Omega_{\lambda}$ we must have $x \underline{E} \subset \underline{E}_{1}^{\prime}$ so that $E^{\prime} \subset E_{1}^{\prime}$. Since $E_{1}^{\prime}$ is irreducible as a $W_{\lambda}^{\prime}$-module we have $E_{1}^{\prime}=E^{\prime}$. This proves (a).

1.18. If $E, \tilde{E} \in \operatorname{Mod} W \mathbf{T}_{n}$ with $E$ irreducible, we set $\tilde{E}^{E}=\operatorname{Hom}_{W \mathbf{T}_{n}}(E, \tilde{E})$. For any $i \geq 0, W \mathbf{T}_{n}$ acts on $S^{i} V \otimes \overline{\mathbf{Q}}_{l}\left[\mathbf{T}_{n}\right]\left(V\right.$ as in 1.17) by $w x_{1}: v \otimes x \mapsto$ $w(v) \otimes w\left(x_{1} x\right)$ (with $w \in W, v \in V, x_{1} \in \mathbf{T}_{n}, x \in \mathbf{T}_{n}$ ). If $E \in \operatorname{Mod} W \mathbf{T}_{n}$ is irreducible, there exists $i \geq 0$ such that $\left(S^{i} V \otimes \overline{\mathbf{Q}}_{l}\left[\mathbf{T}_{n}\right]\right)^{E} \neq 0$. Indeed, there exists $\lambda \in \mathfrak{s}$ and $E_{1} \in \operatorname{Mod} W_{\lambda}^{\prime}$ irreducible such that $E$ is induced by the representation $E_{1} \otimes \lambda$ of the subgroup $W_{\lambda}^{\prime} \mathbf{T}_{n}$. Then $E_{1}$ appears in the $W_{\lambda}^{\prime}$-module

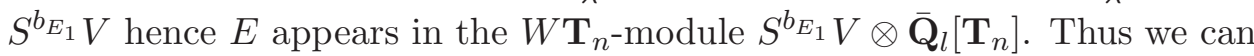
take $i=b_{E_{1}}$. Let $b_{E}$ be the smallest $i \geq 0$ such that $\left(S^{i} V \otimes \overline{\mathbf{Q}}_{l}\left[\mathbf{T}_{n}\right]\right)^{E} \neq 0$. Note that $b_{E} \leq b_{E_{1}}$. Conversely, assume that $\left(S^{i} V \otimes \overline{\mathbf{Q}}_{l}\left[\mathbf{T}_{n}\right]\right)^{E} \neq 0$. Since the $W \mathbf{T}_{n}$-module $S^{i} V \otimes \overline{\mathbf{Q}}_{l}\left[\mathbf{T}_{n}\right]$ is induced by the $W$-module $S^{i} V$ we see (using Frobenius reciprocity) that $\operatorname{dim}\left(S^{i} V \otimes \overline{\mathbf{Q}}_{l}\left[\mathbf{T}_{n}\right]\right)^{E}=\operatorname{dim}_{\operatorname{Hom}}\left(E, S^{i} V\right)$. Since the $W$-module $E$ is induced by the $W_{\lambda}^{\prime}$-module $E_{1}$, the last dimension is equal to $\operatorname{dim}\left(S^{i} V\right)^{E_{1}}$. Thus $\operatorname{dim}\left(S^{i} V\right)^{E_{1}} \neq 0$ so that $b_{E_{1}} \leq i$. We see that $b_{E}=b_{E_{1}}$. This argument shows also that $\left(S^{b_{E}} V \otimes \overline{\mathbf{Q}}_{l}\left[\mathbf{T}_{n}\right]\right)^{E}=\left(S^{b_{E_{1}} V}\right)^{E_{1}}$. We say that $E$ is univalent if $\operatorname{dim}\left(S^{b_{E}} V \otimes \overline{\mathbf{Q}}_{l}\left[\mathbf{T}_{n}\right]\right)^{E}=1$ or equivalently if $E_{1}$ is univalent.

1.19. Let $\mathfrak{A}, I, \mathcal{W}$ be as in 1.13. Thus $\mathcal{W}$ is $W_{\lambda}, W_{\lambda}^{\prime}$ (with $\lambda \in \mathfrak{s}$ ) or $W \mathbf{T}_{n}$. Let sgn be the (one dimensional) sign representation of $W$. The composition of sgn with the obvious homomorphism $\mathcal{W} \rightarrow W$ (the inclusion if $\mathcal{W}$ is $W_{\lambda}$ or $W_{\lambda}^{\prime}$, the projection if $\left.\mathcal{W}=W \mathbf{T}_{n}\right)$ is denoted again by sgn. If $E$ is an irreducible $\mathcal{W}$-module then $E \otimes \operatorname{sgn}$ is again an irreducible $\mathcal{W}$-module. An irreducible representation $E$ of $W$ is said to be special if $a_{E}=b_{E \otimes s g n}$. We show:

(a) If $E$ is an irreducible $\mathcal{W}$-module then $a_{E} \leq b_{E \otimes s g n}$.

(b) For any two-sided cell $\mathbf{c}$ of I there exists a unique (up to isomorphism) irreducible special representation $E$ of $\mathcal{W}$ such that $\mathbf{c}_{E}=\mathbf{c}$. Moreover, $E \otimes \operatorname{sgn}$ is univalent.

In the case where $\mathfrak{A}=\mathbf{H}_{\lambda}$, (a), (b) are known from [10].

We prove (a) for $\mathfrak{A}=\mathbf{H}_{\lambda}^{\prime}$. Let $E$ be an irreducible $W_{\lambda}^{\prime}$-module and let $\mathbf{c}=\mathbf{c}_{E}$ (a two-sided cell of $\left.W_{\lambda}^{\prime}\right)$. Let $E_{0}$ be an irreducible $W_{\lambda}$-module 
appearing in $\left.E\right|_{W_{\lambda}}$ and let $\mathbf{c}_{0}=\mathbf{c}_{E_{0}}$ (a two-sided cell of $W_{\lambda}$ ). We have $\mathbf{c}_{0} \subset \mathbf{c}$ and the $a$-function of $W_{\lambda}$ takes the same value on $\mathbf{c}_{0}$ as the $a$ function of $W_{\lambda}^{\prime}$ on c. Hence $a_{E}=a_{E_{0}}$. Now $E_{0} \otimes \operatorname{sgn}$ appears in $\left.(E \otimes \operatorname{sgn})\right|_{W_{\lambda}}$ hence $b_{E_{0} \otimes \mathrm{sgn}} \leq b_{E \otimes \mathrm{sgn}}$. Since $a_{E_{0}} \leq b_{E_{0} \otimes \mathrm{sgn}}$ is already known we see that $a_{E} \leq b_{E \otimes \text { sgn }}$. Thus (a) holds for $\mathfrak{A}=\mathbf{H}_{\lambda}^{\prime}$.

We prove (b) for $\mathfrak{A}=\mathbf{H}_{\lambda}^{\prime}$. Let $\mathbf{c}$ be a two-sided cell of $W_{\lambda^{\prime}}^{\prime}$. We can find a two-sided cell $\mathbf{c}_{0}$ of $W_{\lambda}$ such that $\mathbf{c}_{0} \subset \mathbf{c}$. We can find an irreducible $W_{\lambda^{-}}$ module $E_{0}$ such that $\mathbf{c}_{E_{0}}=\mathbf{c}_{0}$ and $a_{E_{0}}=b_{E_{0} \otimes \mathrm{sgn}}$. By 1.17(a) we can find an irreducible $W_{\lambda}^{\prime}$-module $E^{\prime}$ such that $E_{0} \otimes \operatorname{sgn}$ appears in $\left.\left(E^{\prime} \otimes \operatorname{sgn}\right)\right|_{W_{\lambda}}$ and $b_{E^{\prime} \otimes \text { sgn }}=b_{E_{0} \otimes \text { sgn }}$ Then $E_{0}$ appears in $\left.E^{\prime}\right|_{W_{\lambda}}$ hence $\mathbf{c}_{E_{0}} \subset \mathbf{c}_{E^{\prime}}$. Thus $\mathbf{c}_{0} \subset \mathbf{c}_{E^{\prime}}$ so that $\mathbf{c}_{E^{\prime}}, \mathbf{c}$ have nonempty intersection and $\mathbf{c}_{E^{\prime}}=\mathbf{c}$; we see also that $a_{E_{0}}=a_{E^{\prime}}$ so that $a_{E^{\prime}}=b_{E^{\prime} \otimes \mathrm{sgn}}$. Thus the existence part of (b) is proved. Assume now that $E^{\prime \prime}$ is an irreducible $W_{\lambda}^{\prime}$-module such that $\mathbf{c}_{E^{\prime \prime}}=\mathbf{c}$ and $a_{E^{\prime \prime}}=b_{E^{\prime \prime} \otimes \mathrm{sgn}}$. Let $E_{1}$ be an irreducible $W_{\lambda}$-module which appears in $\left.E^{\prime \prime}\right|_{W_{\lambda}}$ and let $\mathbf{c}_{1}=\mathbf{c}_{E_{1}}$ so that $\mathbf{c}_{1} \subset \mathbf{c}$ and $a_{E_{1}}=a_{E^{\prime \prime}}$. Replacing $\mathbf{c}_{1}$ by $x \mathbf{c}_{1} x^{-1}$ for some $x \in \Omega_{\lambda}$, we can assume that $\mathbf{c}_{1}=\mathbf{c}_{0}$. Now $E_{1} \otimes \operatorname{sgn}$ appears in $\left.\left(E^{\prime \prime} \otimes \mathrm{sgn}\right)\right|_{W_{\lambda}}$. Hence $b_{E_{1} \otimes \mathrm{sgn}} \leq b_{E^{\prime \prime} \otimes \mathrm{sgn}}=a_{E^{\prime \prime}}=a_{E_{1}}$. Since $a_{E_{1}} \leq b_{E_{1} \otimes \text { sgn }}$ by (a), it follows that $a_{E_{1}}=b_{E_{1} \otimes \mathrm{sgn}}=b_{E^{\prime \prime} \otimes \mathrm{sgn}}$. Similarly we have $a_{E_{0}}=b_{E_{0} \otimes \mathrm{sgn}}$. By the uniqueness in (b) for $W_{\lambda}$ we see that $E_{1} \cong E_{0}$ as $W_{\lambda}$-modules; moreover $E_{0} \otimes \operatorname{sgn}$ is univalent. Now $E_{0} \otimes \operatorname{sgn}$ appears in $\left.\left(E^{\prime} \otimes \mathrm{sgn}\right)\right|_{W_{\lambda}}$ and $b_{E^{\prime} \otimes \mathrm{sgn}}=b_{E_{0} \otimes \mathrm{sgn}} ;$ moreover, $E_{0} \otimes \operatorname{sgn}$ appears in $\left.\left(E^{\prime \prime} \otimes \mathrm{sgn}\right)\right|_{W_{\lambda}}$ and $b_{E^{\prime \prime} \otimes \mathrm{sgn}}=b_{E_{0} \otimes \mathrm{sgn}}$. By the uniqueness in 1.17 (a) we see that $E^{\prime \prime} \otimes \operatorname{sgn} \cong E^{\prime} \otimes \operatorname{sgn}$ so that $E^{\prime \prime} \cong E^{\prime}$; from $1.13($ a) we see also that $E^{\prime \prime} \otimes \operatorname{sgn}$ is univalent. Thus (b) holds for $\mathfrak{A}=\mathbf{H}_{\lambda}^{\prime}$.

We prove (a) for $\mathfrak{A}=\mathbf{H}$. Let $E$ be an irreducible $W \mathbf{T}_{n}$-module and let $\mathbf{c}=\mathbf{c}_{E}$ (a two-sided cell of $\left.W \mathfrak{s}\right)$. We can find $\lambda \in \mathfrak{s}$ such that $1_{\lambda} E \neq 0$. Then $1_{\lambda} E$ is an irreducible $\left(1_{\lambda} \mathbf{H} 1_{\lambda}\right)^{1}$-module hence an irreducible $W_{\lambda}^{\prime}$-module. Let $\mathbf{c}_{1}=\mathbf{c}_{1_{\lambda} E}$, a two-sided cell of $W_{\lambda}^{\prime}$. Then $\left\{w \cdot \lambda ; w \in \mathbf{c}_{1}\right\} \subset \mathbf{c}$ and $a_{\mathbf{c}_{1}}=a_{\mathbf{c}}$ hence $a_{1 \lambda E}=a_{E}$. Now $1_{\lambda}(E \otimes \operatorname{sgn})=\left(1_{\lambda} E\right) \otimes \operatorname{sgn}$ hence by an argument in 1.18 we have $b_{E \otimes \mathrm{sgn}}=b_{\left(1_{\lambda} E\right) \otimes \mathrm{sgn}}$. Since $a_{1_{\lambda} E} \leq b_{\left(1_{\lambda} E\right) \otimes \mathrm{sgn}}$ is already known we see that $a_{E} \leq b_{E \otimes \text { sgn }}$. Thus (a) holds for $\mathfrak{A}=\mathbf{H}$.

We prove (b) for $\mathfrak{A}=\mathbf{H}$. Let $\mathbf{c}$ be a two-sided cell of $W \mathfrak{s}$. Note that $\mathbf{c}$ is also a two-sided cell of $W \times \mathfrak{o}$ for some $W$-orbit $\mathfrak{o}$ in $W \mathfrak{s}$. We can find $\lambda \in \mathfrak{o}$ and a two-sided cell $\mathbf{c}_{0}$ of $W_{\lambda}^{\prime}$ such that $\left\{w \cdot \lambda ; w \in \mathbf{c}_{0}\right\} \subset \mathbf{c}$ and $a_{\mathbf{c}_{0}}=a_{\mathbf{c}}$. We can find an irreducible $W_{\lambda}^{\prime}$-module $E_{0}$ such that $\mathbf{c}_{E_{0}}=\mathbf{c}_{0}$ and $a_{E_{0}}=b_{E_{0} \otimes \text { sgn }}$. Let $E^{\prime}$ be the $W \mathbf{T}_{n}$-module induced by the $W_{\lambda}^{\prime} \mathbf{T}_{n}$-module 
$E_{0} \otimes \lambda$; note that $E^{\prime}$ is irreducible. We have $a_{E^{\prime}}=a_{E_{0}}$. Moreover, $E^{\prime} \otimes \operatorname{sgn}$ is the $W \mathbf{T}_{n}$-module induced by the $W_{\lambda}^{\prime} \mathbf{T}_{n}$-module $\left(E_{0} \otimes \operatorname{sgn}\right) \otimes \lambda$; hence by an argument in 1.18 we have $b_{E^{\prime} \otimes \mathrm{sgn}}=b_{E_{0} \otimes \mathrm{sgn}}$. Thus we have $a_{E^{\prime}}=b_{E^{\prime} \otimes \text { sgn }}$. Thus, the existence part of (b) is proved.

Assume now that $E^{\prime \prime}$ is an irreducible $W \mathbf{T}_{n}$-module such that $\mathbf{c}_{E^{\prime \prime}}=\mathbf{c}$ and $a_{E^{\prime \prime}}=b_{E^{\prime \prime} \otimes \text { sgn }}$. We can find $\lambda^{\prime} \in \mathfrak{s}$ such that $1_{\lambda^{\prime}} E^{\prime \prime} \neq 0$. Since $\mathbf{c}_{E^{\prime \prime}}=\mathbf{c}$ we must have $\lambda^{\prime} \in \mathfrak{o}$. Replacing $\lambda^{\prime}$ by $w\left(\lambda^{\prime}\right)$ for some $w \in W$, we can assume that $\lambda^{\prime}=\lambda$ so that $1_{\lambda} E^{\prime \prime} \neq 0$. Then $\{w \in W ; w \cdot \lambda \in \mathbf{c}\}$ is a two-sided cell of $W_{\lambda}^{\prime}$, necessarily equal to $\mathbf{c}_{0}$; moreover, $\mathbf{c}_{1_{\lambda} E^{\prime \prime}}=\mathbf{c}_{0}$ hence $a_{1 \lambda E^{\prime \prime}}=a_{E^{\prime \prime}}$. Now $E^{\prime \prime}$ is the $W \mathbf{T}_{n}$-module induced by the $W_{\lambda}^{\prime} \mathbf{T}_{n}$-module $\left(1_{\lambda} E^{\prime \prime}\right) \otimes \lambda$ hence $E^{\prime \prime} \otimes$ sgn is the $W \mathbf{T}_{n}$-module induced by the $W_{\lambda}^{\prime} \mathbf{T}_{n}$-module $\left(\left(1_{\lambda} E^{\prime \prime}\right) \otimes \operatorname{sgn}\right) \otimes \lambda$ hence by the argument in 1.18 we have $b_{E^{\prime \prime} \otimes \mathrm{sgn}}=b_{\left(1_{\lambda} E^{\prime \prime}\right) \otimes \mathrm{sgn}}$. It follows that $a_{1_{l} E^{\prime \prime}}=b_{\left(1_{\lambda} E^{\prime \prime}\right) \otimes \mathrm{sgn}}$. Using this and $a_{E_{0}}=b_{E_{0} \otimes \text { sgn }}$ and also the uniqueness part in (b) for $W_{\lambda}^{\prime}$ we see that $E_{0} \cong 1_{\lambda} E^{\prime \prime}$ as $W_{\lambda}^{\prime}$-modules. Since $E^{\prime}$ (resp. $\left.E^{\prime \prime}\right)$ is induced by the $W_{\lambda}^{\prime} \mathbf{T}_{n}$-module $E_{0} \otimes \lambda\left(\operatorname{resp} .\left(1_{\lambda} E^{\prime \prime}\right) \otimes \lambda\right)$ we deduce that $E^{\prime} \cong E^{\prime \prime}$ as $W \mathbf{T}_{n}$-modules. From 1.18 we see also that $E^{\prime \prime}$ is univalent. Thus (b) holds for $\mathfrak{A}=\mathbf{H}$. This completes the proof of (a), (b) in all cases.

The special representation of $\mathcal{W}$ associated to $\mathbf{c}$ in (b) is denoted by $E_{\mathbf{c}}$. It is well defined up to isomorphism. By (b), the special representations of $\mathcal{W}$ (up to isomorphism) are in natural bijection with the two-sided cells of $I$.

1.20. Let $V, S V=\oplus_{i \geq 0} S^{i} V$ be as in 1.17 . For any $i \geq 0$ we set

$$
\mathcal{I}^{i}=\sum_{i^{\prime}>0, i^{\prime \prime} \geq 0 ; i^{\prime}+i^{\prime \prime}=i}\left(S^{i^{\prime}} V\right)^{W} S^{i^{i^{\prime \prime}}} V \subset S^{i} V,
$$

$\bar{S}^{i} V=S^{i} V / \mathcal{I}^{i}$, where $\left(S^{i^{\prime}} V\right)^{W}$ is the space of $W$-invariants in $S^{i^{\prime}} V$ (we have used the algebra structure of $S V)$. Let $\mathcal{I}=\oplus_{i \geq 0} \mathcal{I}^{i}, \bar{S} V=\oplus_{i \geq 0} \bar{S} V^{i}=S V / \mathcal{I}$. Note that $\mathcal{I}$ is an ideal in $S V$ hence $\bar{S} V$ is a (graded) algebra. Note also that the $W$-action on $S V$ preserves $\mathcal{I}$ hence it induces a $W$-action on $\bar{S} V$ which is compatible with the grading and with the algebra structure.

The following property is well known:

(a) $\bar{S}^{i} V=0$ for $i>\nu ; \bar{S}^{\nu}$ is isomorphic to sgn as a $W$-module (in particular it is 1-dimensional). For $i \in[0, \nu]$, the bilinear pairing $\bar{S}^{i} V \times \bar{S}^{\nu-i} \rightarrow \bar{S}^{\nu}$ given by multiplication in $\bar{S} V$ is perfect. 
From (a) we deduce that for $i \in[0, \nu]$ we have $\bar{S}^{i} V \cong \bar{S}^{\nu-i} \otimes \operatorname{sgn}$ as $W$ modules. (We use that any $W$-module is isomorphic to its dual.) Hence if $\lambda \in \mathfrak{s}$ we have

(b) $\bar{S}^{i} V \cong \bar{S}^{\nu-i} \otimes \operatorname{sgn}$ as $W_{\lambda}^{\prime}$-modules. ( $W_{\lambda}^{\prime}$ acts by restriction of the $W$ action.) In particular. for any irreducible representation $E$ of $W_{\lambda}^{\prime}$ we have $\left(\bar{S}^{i} V\right)^{E} \cong\left(\bar{S}^{\nu-i}\right)^{E \otimes \text { sgn }}$.

Clearly, if $E$ is an irreducible $W_{\lambda}^{\prime}$-module and $0 \leq i \leq b_{E}$ then $\left(\mathcal{I}^{i}\right)^{E}=0$ hence $\left(\bar{S}^{i} V\right)^{E}=\left(S^{i} V\right)^{E}$. In particular we have

(c) $\left(\bar{S}^{i} V\right)^{E}=0$ for $0 \leq i<b_{E}$ and $\left(\bar{S}^{b_{E}} V\right)^{E}=\left(S^{b_{E}} V\right)^{E} \neq 0$.

Using (b), (c) we see that:

(c') If $E$ is an irreducible $W_{\lambda}^{\prime}$-module then $\left(\bar{S}^{i} V\right)^{E}=0$ for $i>\nu-b_{E \otimes \operatorname{sgn}}$. Moreover, $\operatorname{dim}\left(\bar{S}^{\nu-b_{E \otimes \operatorname{sgn}}} V\right)^{E}$ is 1 if $E$ is special and $\geq 1$ if $E$ is not special.

Since $a_{E} \leq b_{E \otimes \operatorname{sgn}}$ (see $\left.1.19(\mathrm{a})\right)$ with equality if and only if $E$ is special, we deduce:

(d) If $E$ is an irreducible $W_{\lambda}^{\prime}$-module then $\left(\bar{S}^{i} V\right)^{E}=0$ for $i>\nu-a_{E}$. Moreover, $\operatorname{dim}\left(\bar{S}^{\nu-a_{E}} V\right)^{E}$ is 1 if $E$ is special and 0 if $E$ is not special.

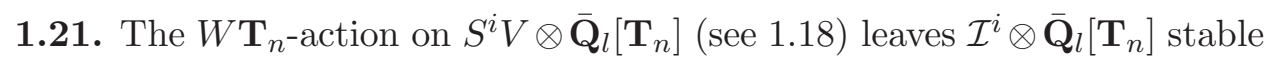
hence it induces a $W \mathbf{T}_{n}$-action on $\bar{S}^{i} V \otimes \overline{\mathbf{Q}}_{l}\left[\mathbf{T}_{n}\right]$. We show:

(a) Let $E$ be an irreducible $W \mathbf{T}_{n}$-module. We have $\left(\bar{S}^{i} V \otimes \overline{\mathbf{Q}}_{l}\left[\mathbf{T}_{n}\right]\right)^{E}=0$ for $i>\nu-a_{E}$. Moreover, $\operatorname{dim}\left(\bar{S}^{\nu-a_{E}} V \otimes \overline{\mathbf{Q}}_{l}\left[\mathbf{T}_{n}\right]\right)^{E}$ is 1 if $E$ is special and 0 if $E$ is not special.

We can find $\lambda \in \mathfrak{s}$ such that $1_{\lambda} E \neq 0$. Then $1_{\lambda} E$ is an irreducible $W_{\lambda^{-}}^{\prime}$ module and $E$ is induced by the representation $\left(1_{\lambda} E\right) \otimes \lambda$ of the subgroup $W_{\lambda}^{\prime} \mathbf{T}_{n}$. Since for $i \geq 0$ the $W \mathbf{T}_{n}$-module $\bar{S}^{i} V \otimes \overline{\mathbf{Q}}_{l}\left[\mathbf{T}_{n}\right]$ is induced by the $W$ module $\bar{S}^{i} V$ we see (using Frobenius reciprocity) that $\operatorname{dim}\left(\bar{S}^{i} V \otimes \overline{\mathbf{Q}}_{l}\left[\mathbf{T}_{n}\right]\right)^{E}=$ $\operatorname{dim} \operatorname{Hom}_{W}\left(E, \bar{S}^{i} V\right)$. Since the $W$-module $E$ is induced by the $W_{\lambda}^{\prime}$-module $1_{\lambda} E$, the last dimension is equal to $\operatorname{dim}\left(\bar{S}^{i} V\right)^{1_{\lambda} E}$. Now (a) follows from 
$1.20(\mathrm{~d})$ applied to $1_{\lambda} E$ instead of $E$, using the equality $a_{E}=a_{1_{\lambda} E}$ and the fact that $E$ is special if and only if $1_{\lambda} E$ is special.

\section{Truncated Convolution of Sheaves on $\tilde{\mathcal{B}}^{2}$}

2.1. For $w \in W$ and $\omega \in \kappa^{-1}(w)$ we define $G_{w} \rightarrow \mathbf{T}, g \mapsto g_{\omega}$, by $g \in$ $\mathbf{U} \omega g_{\omega} \mathbf{U}, g_{\omega} \in \mathbf{T}$. Let $\tilde{\mathcal{B}}=G / \mathbf{U}$. Now $G \times \mathbf{T}^{2}$ acts on $\tilde{\mathcal{B}}^{2}$ by

$$
\left(g, t_{1}, t_{2}\right):(x \mathbf{U}, y \mathbf{U}) \mapsto\left(g x t_{1}^{n} \mathbf{U}, g y t_{2}^{n} \mathbf{U}\right)
$$

The orbits of this action are indexed by $W$ : to $w \in W$ corresponds the orbit $\tilde{\mathcal{O}}_{w}=\left\{(x \mathbf{U}, y \mathbf{U}) \in \tilde{\mathcal{B}}^{2} ; x^{-1} y \in G_{w}\right\}$. The closure of $\tilde{\mathcal{O}}_{w}$ in $\tilde{\mathcal{B}}^{2}$ is $\overline{\tilde{\mathcal{O}}}_{w}=\cup_{y \leq w} \tilde{\mathcal{O}}_{w}$.

Let $w \in W, \omega \in \kappa^{-1}(w)$. We define $j_{\omega}: \tilde{\mathcal{O}}_{w} \rightarrow \mathbf{T}$ by $j_{\omega}(x \mathbf{U}, y \mathbf{U})=$ $\left(x^{-1} y\right)_{\omega}$ Let $\lambda \in \mathfrak{s}$. We set $L_{\lambda}^{\omega}=j_{\omega}^{*} L_{\lambda}$. Now $L_{\lambda}$ is equivariant for the $G \times \mathbf{T}^{2}$ action $\left(g, t_{1}, t_{2}\right): t \mapsto w^{-1}\left(t_{1}\right)^{-n} t t_{2}^{n}$ on $\mathbf{T}$ and this action is compatible under $j_{\omega}$ with the $G \times \mathbf{T}^{2}$-action on $\tilde{\mathcal{O}}_{w}$ (as above); hence $L_{\lambda}^{\omega}$ is a $G \times \mathbf{T}^{2}$-equivariant local system of rank 1 on $\tilde{\mathcal{O}}_{w}$ such that the induced action of $\mathbf{T}_{n}^{2}$ on any stalk is via the character $\left(t_{1}, t_{2}\right) \mapsto w(\lambda)^{-1}\left(t_{1}\right) \lambda\left(t_{2}\right)$. (Note that $\mathbf{T}_{n}^{2}$ acts trivially on $\tilde{\mathcal{B}}^{2}$.) Now let

$$
\Gamma_{w}=\left\{\left(g, t_{0}, t_{1}\right) \in G \times \mathbf{T}^{2} ; g t_{0}^{n} \mathbf{U}=\mathbf{U}, g \omega t_{1}^{n} \mathbf{U}=\omega \mathbf{U}\right\}
$$

be the stabilizer in $G \times \mathbf{T}^{2}$ of $(\mathbf{U}, \omega \mathbf{U}) \in \tilde{\mathcal{O}}_{w}$. Setting $g=t u$ where $t \in \mathbf{T}$, $u \in U_{1}:=\mathbf{U} \cap \omega \mathbf{U} \omega^{-1}$, we can identify

$$
\Gamma_{w}=\left\{\left(u, t, t_{0}, t_{1}\right) \in U_{1} \times \mathbf{T}^{3} ; t t_{0}^{n}=1, w^{-1}(t) t_{1}^{n}=1\right\}
$$

The subgroup

$$
\left\{\left(u, t, t_{0}, t_{1}\right) \in U_{1} \times \mathbf{T}^{3} ; t_{0}=w\left(t_{1}\right), t t_{0}^{n}=1\right\}
$$

of $\Gamma_{w}$ is clearly connected and has the same dimension as $\Gamma_{w}$ (namely $\rho+$ $\operatorname{dim} U_{1}$ ) hence it is the identity component $\Gamma_{w}^{0}$ of $\Gamma_{w}$. We can view $\mathbf{T}_{n}^{2}$ as the kernel of the surjective homomorphism $\Gamma_{w} \rightarrow U_{1} \times \mathbf{T},\left(u, t, t_{0}, t_{1}\right) \mapsto\left(u, t_{0}^{n}\right)$, whose restriction to $\Gamma_{w}^{0}$ must also be surjective. It follows that $\Gamma_{w}=T_{n}^{2} \Gamma_{w}^{0}$ 
hence

$$
\Gamma_{w} / \Gamma_{w}^{0}=\mathbf{T}^{2} /\left(\mathbf{T}^{2} \cap \Gamma_{w}^{0}\right)=\mathbf{T}^{2} /\left\{\left(t_{0}, t_{1}\right) \in \mathbf{T}^{2} ; t_{0}=w\left(t_{1}\right)\right\}
$$

Now the $G \times \mathbf{T}^{2}$-equivariant local systems on $\tilde{\mathcal{O}}_{w}$ correspond to representations of $\Gamma_{w} / \Gamma_{w}^{0}$ hence to representations of $\mathbf{T}_{n}^{2}$ which are trivial on $\left\{\left(t_{1}, t_{2}\right) \in \mathbf{T}_{n}^{2} ; t_{1}=w\left(t_{2}\right)\right\}$. We see that the local systems $L_{\lambda}^{\dot{w}}, \lambda \in \mathfrak{s}$ form a set of representatives for the isomorphism classes of irreducible $G \times \mathbf{T}^{2}$ equivariant local systems on $\tilde{\mathcal{O}}_{w}$.

We define $\tilde{\mathfrak{h}}: \tilde{\mathcal{B}}^{2} \rightarrow \tilde{\mathcal{B}}^{2}$ by $(x \mathbf{U}, y \mathbf{U}) \mapsto(y \mathbf{U}, x \mathbf{U})$. Let $w \in W, \omega \in$ $\kappa^{-1}(w), \lambda \in \mathfrak{s}$. Define $\xi: \mathbf{T} \rightarrow \mathbf{T}$ by $\xi(t)=w\left(t^{-1}\right)$. From the definitions we have $j_{\omega} \tilde{\mathfrak{h}}=\xi j_{\omega^{-1}}: \tilde{\mathcal{O}}_{w^{-1}} \rightarrow \mathbf{T}$. Hence $\tilde{\mathfrak{h}}^{*} L_{\lambda}^{\omega}=L_{w\left(\lambda^{-1}\right)}^{\omega^{-1}}$. (We use that $\xi^{*} L_{\lambda}=L_{w\left(\lambda^{-1}\right)}$.)

2.2. Let $w \in W, \omega \in \kappa^{-1}(w)$. For $l \in \mathfrak{s}$ we shall view $L_{\lambda}^{\omega}$ as a constructible sheaf on $\tilde{\mathcal{B}}^{2}$ which is 0 on $\tilde{\mathcal{B}}^{2}-\tilde{\mathcal{O}}_{w}$. Let $L_{\lambda}^{\omega \sharp}$ be its extension to an intersection cohomology complex on $\overline{\widetilde{\mathcal{O}}}_{w}$ viewed as a complex on $\tilde{\mathcal{B}}^{2}$, equal to 0 on $\tilde{\mathcal{B}}^{2}-$ $\overline{\tilde{\mathcal{O}}}_{w}$. Let $\mathbf{L}_{\lambda}^{\omega}=L_{\lambda}^{\omega \sharp}\langle|w|+\nu+2 \rho\rangle$, a simple perverse sheaf on $\tilde{\mathcal{B}}^{2}$. Note that $L_{\lambda}^{\omega}$ (resp. $\mathbf{L}_{\lambda}^{\omega}$ ) is (noncanonically) isomorphic to $L_{\lambda}^{\dot{w}}$ (resp. $\mathbf{L}_{\lambda}^{\dot{w}}$ ). (We use 1.5). We have

$$
\tilde{\mathfrak{h}}^{*} \mathbf{L}_{\lambda}^{\omega}=\mathbf{L}_{w\left(\lambda^{-1}\right)}^{\omega^{-1}}
$$

2.3. For $i<j$ in $[0,2]$ we define $p_{i j}: \tilde{\mathcal{B}}^{3} \rightarrow \tilde{\mathcal{B}}^{2}$ by $\left(x_{0} \mathbf{U}, x_{1} \mathbf{U}, x_{2} \mathbf{U}\right) \mapsto$ $\left(x_{i} \mathbf{U}, x_{j} \mathbf{U}\right)$. For $L, L^{\prime}$ in $\mathcal{D}\left(\tilde{\mathcal{B}}^{2}\right)$ we set $L \circ L^{\prime}=p_{02 !}\left(p_{01}^{*} L \otimes p_{12}^{*} L^{\prime}\right) \in \mathcal{D}\left(\tilde{\mathcal{B}}^{2}\right)$. This operation is associative. Hence for ${ }^{1} L,{ }^{2} L, \ldots,{ }^{m} L$ in $\mathcal{D}\left(\tilde{\mathcal{B}}^{2}\right),{ }^{1} L \circ{ }^{2} L \circ$ $\ldots{ }^{m} L \in \mathcal{D}\left(\tilde{\mathcal{B}}^{2}\right)$ is defined.

We have $p_{02}=\bar{p}_{02} f$ where $f: \tilde{\mathcal{B}}^{3} \rightarrow \tilde{\mathcal{B}} \times \mathcal{B} \times \tilde{\mathcal{B}}$ is $\left(x_{0} \mathbf{U}, x_{1} \mathbf{U}, x_{2} \mathbf{U}\right) \mapsto$ $\left(x_{0} \mathbf{U}, x_{1} \mathbf{B} x_{1}^{-1}, x_{2} \mathbf{U}\right)$ and $\bar{p}_{02}: \tilde{\mathcal{B}} \times \mathcal{B} \times \tilde{\mathcal{B}} \rightarrow \tilde{\mathcal{B}}^{2}$ is $\left(x_{0} \mathbf{U}, B, x_{2} \mathbf{U}\right) \mapsto\left(x_{0} \mathbf{U}\right.$, $\left.x_{2} \mathbf{U}\right)$. We show:

(a) Let $w, w^{\prime} \in W, \omega \in \kappa^{-1}(w), \omega^{\prime} \in \kappa^{-1}\left(w^{\prime}\right), \lambda, \lambda^{\prime} \in \mathfrak{s}$. If $w^{\prime}\left(\lambda^{\prime}\right) \neq \lambda$ then $L_{\lambda}^{\omega} \circ L_{\lambda^{\prime}}^{\omega^{\prime}}=0$. 
It is enough to show that $f_{!}\left(p_{01}^{*} L_{\lambda}^{\omega} \otimes p_{12}^{*} L_{\lambda^{\prime}}^{\omega^{\prime}}\right)=0$. Hence it is enough to show that for any $\left(x_{0}, x_{1}, x_{2}\right) \in G^{3}$ and any $i$ we have

$$
H_{c}^{i}\left(f^{-1}\left(x_{0} \mathbf{U}, x_{1} \mathbf{B} x_{1}^{-1}, x_{2} \mathbf{U}\right), p_{01}^{*} L_{\lambda}^{\omega} \otimes p_{12}^{*} L_{\lambda^{\prime}}^{\omega^{\prime}}\right)=0
$$

We have

$$
f^{-1}\left(x_{0} \mathbf{U}, x_{1} \mathbf{B} x_{1}^{-1}, x_{2} \mathbf{U}\right)=\left\{\left(x_{0} \mathbf{U}, x_{1} \tau \mathbf{U}, x_{2} \mathbf{U}\right) ; \tau \in \mathbf{T}\right\}
$$

hence this fibre of $f$ is empty unless $\xi_{0}^{-1} x_{1} \in \mathbf{U} \omega t_{0} \mathbf{U}, x_{1}^{-1} x_{2} \in \mathbf{U} \omega^{\prime} t_{0}^{\prime} \mathbf{U}$ for some $t_{0}, t_{0}^{\prime}$ in $\mathbf{T}$ (which we now assume) so that the fibre can be identified with $\mathbf{T}$. The restriction of $p_{01}$ (resp. $p_{12}$ to this fibre can be identified with $\tau \mapsto t_{0} \tau$ (resp. $\left.\tau \mapsto w^{\prime-1}\left(\tau^{-1}\right) t_{0}^{\prime}\right)$. Then $p_{01}^{*} L_{\lambda}^{\omega} \otimes p_{12}^{*} L_{\lambda^{\prime}}^{\omega^{\prime}}$ becomes the local system $L_{\lambda} \otimes L_{w^{\prime}\left(\lambda^{\prime}\right)^{-1}}=L_{\lambda w^{\prime}\left(\lambda^{\prime}\right)^{-1}}$ on $\mathbf{T}$. It remains to use that $H_{c}^{i}\left(\mathbf{T}, L_{\lambda_{1}}\right)=0$ if $\lambda_{1} \in \mathfrak{s}-\{1\}$.

2.4. Let $w, w^{\prime} \in W$ be such that $\left|w w^{\prime}\right|=|w|+\left|w^{\prime}\right|$, let $\omega \in \kappa^{-1}(w), \omega^{\prime} \in$ $\kappa^{-1}\left(w^{\prime}\right)$ and let $\lambda, \lambda^{\prime} \in \mathfrak{s}$. We show:

(a) If $w^{\prime}\left(\lambda^{\prime}\right)=\lambda$, then we have canonically $L_{\lambda}^{\omega} \circ L_{\lambda^{\prime}}^{\omega^{\prime}}=L_{\lambda^{\prime}}^{\omega \omega^{\prime}} \otimes \mathfrak{L}$.

Let

$$
Y=\left\{\left(x \mathbf{U}, y \mathbf{U}, t, t^{\prime}\right) \in \tilde{\mathcal{B}} \times \tilde{\mathcal{B}} \times \mathbf{T} \times \mathbf{T} ; x^{-1} y \in \mathbf{U} \omega \omega^{\prime} w^{\prime-1}(t) t^{\prime} \mathbf{U}\right\}
$$

Define $h: \mathbf{T}^{2} \rightarrow \mathbf{T}$ by $h\left(t, t^{\prime}\right)=w^{\prime-1}(t) t^{\prime}$. Define $j: Y \rightarrow \tilde{\mathcal{O}}_{w w^{\prime}}$ by

$$
j\left(x \mathbf{U}, y \mathbf{U}, t, t^{\prime}\right)=(x \mathbf{U}, y \mathbf{U})
$$

Define $j_{1}: Y \rightarrow \mathbf{T}$ by $j_{1}\left(x \mathbf{U}, y \mathbf{U}, t, t^{\prime}\right)=\left(t, t^{\prime}\right)$. Let $j^{\prime}=j_{\omega \omega^{\prime}}: \tilde{\mathcal{O}}_{w w^{\prime}} \rightarrow \mathbf{T}$ be as in 2.1. From the definitions we have

$$
L_{\lambda}^{\omega} \circ L_{\lambda^{\prime}}^{\omega^{\prime}}=j_{!}\left(j_{1}^{*}\left(L_{\lambda} \otimes L_{\lambda^{\prime}}\right)\right)=j^{* *}\left(h_{!}\left(L_{\lambda} \otimes L_{\lambda^{\prime}}\right)\right) .
$$

To prove (a) it remains to show that $h_{!}\left(L_{\lambda} \otimes L_{\lambda^{\prime}}\right)=L_{\lambda^{\prime}} \otimes \mathfrak{L}$. Replacing $\lambda$ by $w^{\prime-1}(\lambda)$ and $h$ by $h^{\prime}: \mathbf{T}^{2} \rightarrow \mathbf{T}, h^{\prime}\left(t, t^{\prime}\right)=t t^{\prime}$ we see that it is enough to show that $h_{!}^{\prime}\left(L_{\lambda} \otimes L_{\lambda}\right)=L_{\lambda} \otimes \mathfrak{L}$. We have $h_{!}\left(L_{\lambda} \otimes L_{\lambda}\right)=h_{!}^{\prime} h^{\prime *} L_{\lambda}$ and it remains to use the equality $h_{!}^{\prime} \overline{\mathbf{Q}}_{l}=\mathfrak{L}$. 
2.5. Let $s \in S, \lambda^{\prime} \in \mathfrak{s}$. Let $L^{\prime}$ be the local system of rank 1 on $\tilde{\mathcal{B}}^{2} \times\left(\mathbf{U}_{s}-\{1\}\right)$ whose restriction to $\tilde{\mathcal{B}}^{2} \times\{\xi\}$ is $L_{\lambda^{\prime}}^{\sigma_{\xi}}$ for any $\xi \in \mathbf{U}_{s}-\{1\}$ (see 1.2). Let $\hat{L}_{\lambda^{\prime}}^{s}=c_{!} L^{\prime} \in \mathcal{D}\left(\tilde{\mathcal{B}}^{2}\right)$ where $c: \tilde{\mathcal{B}}^{2} \times\left(\mathbf{U}_{s}-\{1\}\right) \rightarrow \tilde{\mathcal{B}}^{2}$ is the obvious projection. Clearly, we have

$$
\hat{L}_{\lambda^{\prime}}^{s} \approx\left\{\mathcal{H}^{2} \hat{L}_{\lambda^{\prime}}^{s}[-2], \mathcal{H}^{1} \hat{L}_{\lambda^{\prime}}^{s}[-1]\right) .
$$

Moreover, if $s \notin W_{\lambda^{\prime}}$ then $\mathcal{H}^{2} \hat{L}_{\lambda^{\prime}}^{s}[-2]=0, \mathcal{H}^{1} \hat{L}_{\lambda^{\prime}}^{s}[-1]=0$ hence $\hat{L}_{\lambda^{\prime}}^{s}=0$. If $s \in W_{\lambda^{\prime}}$ then $\mathcal{H}^{2} \hat{L}_{\lambda^{\prime}}^{s}[-2]=L_{\lambda^{\prime}}^{\dot{s}}[-2](-1), \mathcal{H}^{1} \hat{L}_{\lambda^{\prime}}^{s}[-2]=L_{\lambda^{\prime}}^{\dot{s}}[-1]$.

2.6. Let $s \in S$ and let $\lambda, \lambda^{\prime} \in \mathfrak{s}$ be such that $s\left(\lambda^{\prime}\right)=\lambda$. From the definitions we see that:

$$
L_{\lambda}^{\dot{s}} \circ L_{\lambda^{\prime}}^{\dot{s}^{-1}} \approx\left\{L_{\lambda^{\prime}}^{1}[-2](-1) \otimes \mathfrak{L}, \hat{L}_{\lambda^{\prime}}^{s}\right\}
$$

Using the results in 2.5 we deduce:

(a) If $s \notin W_{\lambda}$, then $L_{\lambda}^{\dot{s}} \circ L_{\lambda^{\prime}}^{\dot{s}^{-1}}=L_{\lambda^{\prime}}^{1}[-2](-1) \otimes \mathfrak{L}$.

(b) If $s \in W_{\lambda}$, then

$$
L_{\lambda}^{\dot{s}} \circ L_{\lambda^{\prime}}^{\dot{s}^{-1}} \approx\left\{L_{\lambda^{\prime}}^{1}[-2](-1) \otimes \mathfrak{L}, L_{\lambda^{\prime}}^{\dot{s}}[-2](-1) \otimes \mathfrak{L}, L_{\lambda^{\prime}}^{\dot{s}}[-1] \otimes \mathfrak{L}\right\} .
$$

(Note that the conditions $s \in W_{\lambda}$ and $s \in W_{\lambda^{\prime}}$ are equivalent.)

2.7. Let $s \in S, w \in W$ be such that $|s w|<|w|$ and let $\omega \in \kappa^{-1}(w), \lambda, \lambda^{\prime} \in \mathfrak{s}$ be such that $w\left(\lambda^{\prime}\right)=\lambda$. We show:

(a) If $s \notin W_{\lambda}$ then $L_{\lambda}^{\dot{s}} \circ L_{\lambda^{\prime}}^{\omega} \otimes \mathfrak{L}=L_{\lambda^{\prime}}^{\dot{s} \omega}[-2](-1) \otimes \mathfrak{L} \otimes \mathfrak{L}$.

(b) If $s \in W_{\lambda}$, then

$L_{\lambda}^{\dot{s}} \circ L_{\lambda^{\prime}}^{\omega} \otimes \mathfrak{L} \approx\left\{L_{\lambda^{\prime}}^{\dot{s} \omega}[-2](-1) \otimes \mathfrak{L}^{\otimes 2}, L_{\lambda^{\prime}}^{\omega}[-2](-1) \otimes \mathfrak{L}^{\otimes 2}, L_{\lambda^{\prime}}^{\omega}[-1] \otimes \mathfrak{L}^{\otimes 2}\right\}$.

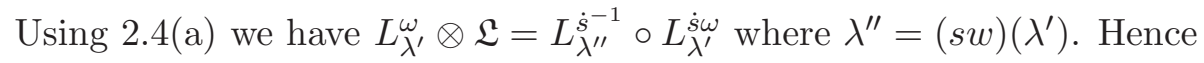

$$
L_{\lambda}^{\dot{s}} \circ L_{\lambda^{\prime}}^{\omega} \otimes \mathfrak{L}=L_{\lambda}^{\dot{s}} \circ L_{\lambda^{\prime \prime}}^{\dot{s}^{-1} \circ L_{\lambda^{\prime}}^{\dot{s} \omega}}
$$

We now apply the results in 2.6 to describe $L_{\lambda}^{\dot{s}} \circ L_{\lambda^{\prime \prime}}^{\dot{s}^{-1}}$. In case (a), we obtain

$$
L_{\lambda}^{\dot{s}} \circ L_{\lambda^{\prime}}^{\omega} \otimes \mathfrak{L}=L_{\lambda^{\prime \prime}}^{1} \circ L_{\lambda^{\prime}}^{\dot{s} \omega} \otimes \mathfrak{L}[-2](-1) .
$$


By 2.4(a) this equals $L_{\lambda^{\prime}}^{\dot{s} \omega} \otimes \mathfrak{L}^{\otimes}[2](-1)$, proving (a). In case (b) we obtain

$$
\begin{aligned}
& L_{\lambda}^{\dot{s}} \circ L_{\lambda^{\prime}}^{\omega} \otimes \mathfrak{L} \approx
\end{aligned}
$$

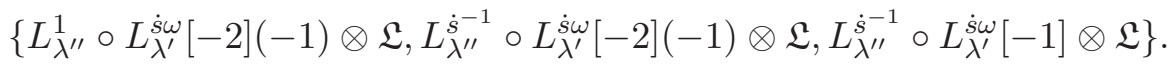

Here we substitute $L_{\lambda^{\prime \prime}}^{1} \circ L_{\lambda^{\prime}}^{\dot{s} \omega}=L_{\lambda^{\prime}}^{\dot{s} \omega} \otimes \mathfrak{L}, L_{\lambda^{\prime \prime}}^{\dot{s}} \circ L_{\lambda^{\prime}}^{\dot{s} \omega}=L_{\lambda^{\prime}}^{\omega} \otimes \mathfrak{L}($ see $2.4(\mathrm{a}))$ and (b) follows.

2.8. We choose an $\mathbf{F}_{q}$-rational structure on $G$. We shall assume that $\mathbf{B}$ (hence $\mathbf{U}$ ) is defined over $\mathbf{F}_{q}$, that $\mathbf{T}$ is defined and split over $\mathbf{F}_{q}$ and that the integer $n$ in 1.4 divides $q-1$. Then for each $s \in S$, the subgroup $\mathbf{U}_{s}$ is defined over $\mathbf{F}_{q}$; we shall also assume that in 1.3 we have $\xi_{s} \in \mathbf{U}_{s}\left(\mathbf{F}_{q}\right)-\{1\}$. We have induced $\mathbf{F}_{q}$-structures on $\mathcal{B}, \tilde{\mathcal{B}}$. For any $w \in W, \mathcal{O}_{w}, \overline{\mathcal{O}}_{w}, \tilde{\mathcal{O}}_{w}, \overline{\mathcal{O}}_{w}$ inherit natural $\mathbf{F}_{q}$-structures. For any $w \in W$ we write $\kappa_{q}^{-1}(w)$ instead of $\kappa^{-1}(w) \cap G\left(\mathbf{F}_{q}\right)$; note that $\dot{w} \in \kappa_{q}^{-1}(w),(\dot{w})^{-1} \in \kappa_{q}^{-1}\left(w^{-1}\right)$. Now the local system $\iota_{!} \overline{\mathbf{Q}}_{l}$ in 1.4 is naturally pure of weight zero (since $\overline{\mathbf{Q}}_{l}$ is so) and each of its direct summands $L_{\lambda}$ is itself naturally pure of weight zero (since $n$ divides $q-1)$. If $\omega \in \kappa_{q}^{-1}(w)$, it follows that the local system $L_{\lambda}^{\omega}$ on $\tilde{\mathcal{O}}_{w}$ is naturally pure of weight zero. Hence $L_{\lambda}^{\omega \sharp}, \mathbf{L}_{\lambda}^{\omega}$ are naturally pure of weight zero. In particular, $L_{\lambda}^{\dot{w}}, L_{\lambda}^{\dot{w} \sharp}, \mathbf{L}_{\lambda}^{\dot{w}}$ are naturally pure of weight zero.

Let $\mathcal{D}^{\mathcal{B}^{2}}$ be the subcategory of $\mathcal{D}\left(\tilde{\mathcal{B}}^{2}\right)$ consisting of objects which are restrictions of objects in the $G \times \mathbf{T}^{2}$-equivariant derived category. Let $\mathcal{D}_{m}^{\mathcal{B}^{2}}$ be the subcategory of $\mathcal{D}_{m}\left(\tilde{\mathcal{B}}^{2}\right)$ consisting of objects which are restrictions of objects in the mixed $G \times \mathbf{T}^{2}$-equivariant derived category. Let $\mathcal{M}^{\wedge} \tilde{\mathcal{B}}^{2}$ (resp. $\mathcal{M}_{m}^{\infty} \tilde{\mathcal{B}}^{2}$ ) be the subcategory of $\mathcal{D}^{\tilde{\mathcal{B}}^{2}}$ (resp. $\mathcal{D}_{m}^{\mathbf{B}} \tilde{\mathcal{B}}^{2}$ ) consisting of objects which are perverse sheaves.

If $w \in W, \omega \in \kappa_{q}^{-1}(w)$ then $L_{\lambda}^{\omega}$ (resp. $L_{\lambda}^{\omega \sharp}, \mathbf{L}_{\lambda}^{\omega}$ ) is (noncanonically) isomorphic to $L_{\lambda}^{\dot{w}}\left(\operatorname{resp} . L_{\lambda}^{\dot{w} \sharp}, \mathbf{L}_{\lambda}^{\dot{w}}\right)$ as objects of $\mathcal{D}_{m}\left(\tilde{\mathcal{B}}^{2}\right)$.

2.9. Let $L \in \mathcal{D}_{m}^{\mathbf{N}}\left(\tilde{\mathcal{B}}^{2}\right)$. For any $w \in W, i \in \mathbf{Z},\left.\mathcal{H}^{i} L\right|_{\tilde{\mathcal{O}}_{w}}$ is a $G \times \mathbf{T}^{2}$-equivariant local system with an induced mixed structure. We can write it as

$$
\left.\mathcal{H}^{i} L\right|_{\tilde{\mathcal{O}}_{w}}=\oplus_{\lambda \in \mathfrak{s}} V_{L, i, w, \lambda} \otimes L_{\lambda}^{\dot{w}}
$$


where $V_{L, i, w, \lambda}$ are mixed $\overline{\mathbf{Q}}_{l}$-vector spaces. For $j \in \mathbf{Z}$ let $V_{L, i, w, \lambda, j}$ be the subquotient of $V_{L, i, w, \lambda, j}$ of pure weight $j$. We set

$$
\gamma(L)=\sum_{w \in W, \lambda \in \mathfrak{s}} \sum_{i, j \in \mathbf{Z}}(-1)^{i}(-v)^{j} \operatorname{dim} V_{L, i, w, \lambda, j} T_{w} 1_{\lambda} \in \mathbf{H} .
$$

For example, if $w \in W$ and $\omega \in \kappa_{q}^{-1}(w), \lambda \in \mathfrak{s}$ then

$$
\gamma\left(L_{\lambda}^{\omega}\right)=\gamma\left(L_{\lambda}^{\dot{w}}\right)=T_{w} 1_{\lambda}
$$

Note that

(a) if $\left(L, L^{\prime}, L^{\prime \prime}\right)$ is a distinguished triangle in $\mathcal{D}_{m}^{\mathbf{A}}\left(\tilde{\mathcal{B}}^{2}\right)$, then $\gamma\left(L^{\prime}\right)=\gamma(L)+$ $\gamma\left(L^{\prime \prime}\right)$.

2.10. Let $w, w^{\prime} \in W, \omega \in \kappa_{q}^{-1}(w), \omega^{\prime} \in \kappa_{q}^{-1}\left(w^{\prime}\right), \lambda, \lambda^{\prime} \in \mathfrak{s}$. We show:

$$
\gamma\left(L_{\lambda}^{\omega} \circ L_{\lambda^{\prime}}^{\omega^{\prime}}\right)=\left(v^{2}-1\right)^{\rho} \gamma\left(L_{\lambda}^{\omega}\right) \gamma\left(L_{\lambda^{\prime}}^{\omega^{\prime}}\right)
$$

The right hand side of (a) is $\left(v^{2}-1\right)^{\rho} T_{w} 1_{\lambda} T_{w^{\prime}} 1_{\lambda^{\prime}}$. We prove (a) by induction on $|w|$. If $|w|=0$ then by 2.4(a), 2.3(a), the left hand side of (a) is $\left(v^{2}-\right.$ 1) ${ }^{\rho} T_{w^{\prime}} 1_{\lambda^{\prime}}$ (if $w^{\prime}\left(\lambda^{\prime}\right)=\lambda$ ) and 0 otherwise; this is clearly equal to the right hand side of (a). Now assume that $|w| \geq 1$. We can find $s \in S$ such that $|w|=|w s|+1$. The right hand side of (a) is

$$
\left(v^{2}-1\right)^{\rho} T_{w} 1_{\lambda} T_{w^{\prime}} 1_{\lambda^{\prime}}=\left(v^{2}-1\right)^{\rho} T_{w s} T_{s} 1_{\lambda} T_{w^{\prime}} 1_{\lambda^{\prime}} .
$$

If $w^{\prime}\left(\lambda^{\prime}\right) \neq \lambda$ then (b) is 0. If $w^{\prime}\left(\lambda^{\prime}\right)=\lambda$ and $\left|s w^{\prime}\right|=\left|w^{\prime}\right|+1$ then (b) is $\left(v^{2}-1\right)^{\rho} T_{w s} T_{s w^{\prime}} 1_{\lambda^{\prime}}$. If $w^{\prime}\left(\lambda^{\prime}\right)=\lambda$ and $\left|s w^{\prime}\right|=\left|w^{\prime}\right|-1, s \notin W_{\lambda}$ then (b) is $v^{2}\left(v^{2}-1\right)^{\rho} T_{w s} T_{s w^{\prime}} 1_{\lambda^{\prime}}$. If $w^{\prime}\left(\lambda^{\prime}\right)=\lambda$ and $\left|s w^{\prime}\right|=\left|w^{\prime}\right|-1, s \in W_{\lambda}$ then (b) is

$$
\left(v^{2}-1\right)^{\rho} T_{w s}\left(v^{2} T_{s w^{\prime}}+\left(v^{2}-1\right) T_{w^{\prime}}\right) 1_{\lambda^{\prime}}
$$

Let $\lambda^{\prime \prime}=s(\lambda)$. By $2.4\left(\right.$ a) we have $L_{\lambda}^{\omega} \otimes \mathfrak{L}=L_{\lambda^{\prime \prime}}^{\omega \dot{s}^{-1}} \circ L_{\lambda}^{\dot{s}}$, hence

$$
\mathfrak{K}:=L_{\lambda}^{\omega} \circ L_{\lambda^{\prime}}^{\omega^{\prime}} \otimes \mathfrak{L} \otimes \mathfrak{L}=L_{\lambda^{\prime \prime}}^{\omega \dot{s}^{-1}} \circ L_{\lambda}^{\dot{s}} \circ L_{\lambda^{\prime}}^{\omega^{\prime}} \otimes \mathfrak{L} .
$$

If $w^{\prime}\left(\lambda^{\prime}\right) \neq \lambda$, then $\mathfrak{K}=0$ by $2.3(\mathrm{a})$; hence in this case (a) holds. Thus we 
can assume that $w^{\prime}\left(\lambda^{\prime}\right)=\lambda$. If $\left|s w^{\prime}\right|=\left|w^{\prime}\right|+1$ we have (using 2.4(a))

$$
\mathfrak{K}=L_{\lambda^{\prime \prime}}^{\omega \dot{s}^{-1}} \circ L_{\lambda^{\prime}}^{\dot{s} \omega^{\prime}} \otimes \mathfrak{L} \otimes \mathfrak{L}
$$

hence by the induction hypothesis

$$
\left.\left(v^{2}-1\right)^{2 \rho} \gamma\left(L_{\lambda}^{\omega}\right) \circ L_{\lambda^{\prime}}^{\omega^{\prime}}\right)=\left(v^{2}-1\right)^{3 \rho} T_{w s} 1_{\lambda^{\prime \prime}} T_{s w^{\prime}} 1_{\lambda^{\prime}}
$$

hence in this case (a) holds. We now assume that $w^{\prime}\left(\lambda^{\prime}\right)=\lambda,\left|s w^{\prime}\right|=\left|w^{\prime}\right|-1$. Using $2.7(\mathrm{a}),(\mathrm{b})$ to describe $L_{\lambda}^{\dot{s}} \circ L_{\lambda^{\prime}}^{\omega^{\prime}} \otimes \mathfrak{L}$ we deduce that

$$
\begin{array}{rlr}
\mathfrak{K}= & L_{\lambda^{\prime \prime}}^{\omega \dot{s}^{-1}} \circ L_{\lambda^{\prime}}^{\dot{s} \omega^{\prime}} \otimes \mathfrak{L}^{\otimes 2}[-2](-1) & \text { if } s \notin W_{\lambda}, \\
\mathfrak{K} \approx & \left\{L_{\lambda^{\prime \prime}}^{\omega \dot{s}^{-1}} \circ L_{\lambda^{\prime}}^{\dot{s} \omega^{\prime}} \otimes \mathfrak{L}^{\otimes 2}[-2](-1), L_{\lambda^{\prime \prime}}^{\omega \dot{s}^{-1}} \circ L_{\lambda^{\prime}}^{\omega^{\prime}} \otimes \mathfrak{L}^{\otimes 2}[-2](-1),\right. & \text { if } s \in W_{\lambda} . \\
& \left.L_{\lambda^{\prime \prime}}^{\omega \dot{s}^{-1}} \circ L_{\lambda^{\prime}}^{\dot{s} \omega^{\prime}} \otimes \mathfrak{L}^{\otimes 2}[-1]\right\}
\end{array}
$$

It follows that

$$
\begin{array}{lr}
\gamma(\mathfrak{K})=v^{2}\left(v^{2}-1\right)^{2 \rho} \gamma\left(L_{\lambda^{\prime \prime}}^{\omega \dot{s}^{-1}} \circ L_{\lambda^{\prime}}^{\dot{s} \omega^{\prime}}\right) & \text { if } s \notin W_{\lambda}, \\
\gamma(\mathfrak{K})=\left(v^{2}-1\right)^{2 \rho}\left(v^{2} \gamma\left(L_{\lambda^{\prime \prime}}^{\omega \dot{s}^{-1}} \circ L_{\lambda^{\prime}}^{\dot{s} \omega^{\prime}}\right)+\left(v^{2}-1\right) \gamma\left(L_{\lambda^{\prime \prime}}^{\omega \dot{s}^{-1}} \circ L_{\lambda^{\prime}}^{\omega^{\prime}}\right)\right) & \text { if } s \in W_{\lambda} .
\end{array}
$$

Using the induction hypothesis we see that

$$
\begin{array}{ll}
\gamma(\mathfrak{K})=v^{2}\left(v^{2}-1\right)^{3 \rho} T_{w s} 1_{\lambda^{\prime \prime}} T_{s w^{\prime}} 1_{\lambda^{\prime}} & \text { if } s \notin W_{\lambda}, \\
\gamma(\mathfrak{K})=\left(v^{2}-1\right)^{3 \rho}\left(v^{2} T_{w s} 1_{\lambda^{\prime \prime}} T_{s w^{\prime}} 1_{\lambda^{\prime}}+\left(v^{2}-1\right) T_{w s} 1_{\lambda^{\prime \prime}} T_{w^{\prime}} 1_{\lambda^{\prime}}\right) & \text { if } s \in W_{\lambda} .
\end{array}
$$

Thus, (a) holds.

2.11. Let $r \geq 1$ and let ${ }^{1} L,{ }^{2} L, \ldots,{ }^{r} L$ be objects of $\mathcal{D}_{m}^{\wedge} \tilde{\mathcal{B}}^{2}$. We show:

$$
\gamma\left({ }^{1} L \circ{ }^{2} L \circ \ldots \circ{ }^{r} L\right)=\left(v^{2}-1\right)^{(r-1) \rho} \gamma\left({ }^{1} L\right) \gamma\left({ }^{2} L\right) \ldots \gamma\left({ }^{r} L\right) .
$$

When $r=1$, (a) is obvious. For $r \geq 2$, (a) follows easily by induction from the case when $r=2$. Thus we may assume that $r=2$. For $j=1,2$ we have

$$
{ }^{j} L \approx\left\{\mathcal{H}^{i}\left({ }^{j} L\right)[-i] ; i \in \mathbf{Z}\right\}
$$

hence (using 2.9(a)), $\gamma\left({ }^{j} L\right)=\sum_{i}(-1)^{i} \gamma\left(\mathcal{H}^{i}\left({ }^{j} L\right)\right)$. Moreover,

$$
{ }^{1} L \circ{ }^{2} L \approx\left\{\mathcal{H}^{i}\left({ }^{1} L\right) \circ \mathcal{H}^{i^{\prime}}\left({ }^{2} L\right)\left[-i-i^{\prime}\right] ; i, i^{\prime} \in \mathbf{Z}\right\}
$$


hence (using 2.9(a)),

$$
\gamma\left({ }^{1} L \circ{ }^{2} L\right)=\sum_{i, i^{\prime} \in \mathbf{Z}}(-1)^{i+i^{\prime}} \gamma\left(\mathcal{H}^{i}\left({ }^{1} L\right) \circ \mathcal{H}^{i^{\prime}}\left({ }^{2} L\right)\right) .
$$

Thus we can assume that ${ }^{1} L=L_{\lambda}^{\dot{w}},{ }^{2} L=L_{\lambda^{\prime}}^{\dot{w}^{\prime}}$ where $w, w^{\prime} \in W, \lambda, \lambda^{\prime} \in \mathfrak{s}$. In this case, (a) follows from 2.10(a).

2.12. Let $\lambda \in \mathfrak{s}$. We choose for each $\eta \in W_{\lambda}$ an element $\ddot{\eta} \in \kappa^{-1}(\eta)$ as follows. Assume first that $|\eta|_{\lambda}=1$. We write $\eta=s_{1} s_{2} \ldots s_{r} s_{r+1} s_{r} \ldots s_{1}$ with $s_{1}, s_{2} \ldots, s_{r+1}$ in $S$; we set

$$
\ddot{\eta}=\dot{s}_{1} \dot{s}_{2} \ldots \dot{s}_{r} \dot{s}_{r+1} \dot{s}_{r}^{-1} \ldots \dot{s}_{1}^{-1}
$$

Assume next that $|\eta|_{\lambda}=m$. We write $\eta=\eta_{1} \eta_{2} \ldots \eta_{m}$ with $\eta_{i} \in W_{\lambda}$ such that $\left|\eta_{1}\right|_{\lambda}=\cdots=\left|\eta_{m}\right|_{\lambda}=1,|\eta|_{\lambda}=m$ and we set $\ddot{\eta}=\ddot{\eta}_{1} \ddot{\eta}_{2} \ldots \ddot{\eta}_{m}$. (In particular, $\ddot{1}=1$.)

We now define for each $w \in W$ an element $\ddot{w} \in \kappa_{q}^{-1}(w)$ as follows. There is a unique $z \in W$ such that $z=\min \left(w W_{\lambda}\right)$. We have $w=z \eta$ for a unique $\eta \in W_{\lambda}$. We set $\ddot{w}=\dot{z} \ddot{\eta}$.

Let $w, y \in W$. Let $z=\min \left(w W_{\lambda}\right)$. We write $w=z \eta$ with $\eta \in W_{\lambda}$. Let $i \in \mathbf{Z}$. The statements (a), (b) below can be deduced from [13, 1.24] in the same way as [16, 12.4] was deduced from [13, 1.24].

(a) We have $\left.\mathcal{H}^{i} L_{\lambda}^{\ddot{w} \sharp}\right|_{\tilde{\mathcal{O}}_{y}}=0$ unless $i$ is even and $y \in w W_{\lambda}$.

(b) Assume that $i$ is even and $y \in w W_{\lambda}$. We write $y=z \eta^{\prime}$ with $\eta^{\prime} \in W_{\lambda}$.

We have

$$
\left.\mathcal{H}^{i} L_{\lambda}^{\ddot{w} \sharp}\right|_{\tilde{\mathcal{O}}_{y}} \approx\left\{\left(L_{\lambda}^{\ddot{y}}\right)_{h}(-i / 2) ; h \in\left[1, n_{\lambda, \eta^{\prime}, \eta, i}\right]\right\}
$$

where $\left(L_{\lambda}^{\ddot{y}}\right)_{h}$ are copies of $L_{\lambda}^{\ddot{y}}$ and $n_{\lambda, \eta^{\prime}, \eta, i}$ is the coefficient of $X^{i / 2}$ in

$$
X^{(1 / 2)\left(|w|-|y|-\left.|\eta|_{\lambda}|+| \eta^{\prime}\right|_{\lambda}\right)} P_{\eta^{\prime}, \eta}^{\lambda}(X),
$$

see 1.8 .

From (a), (b) we deduce:

$$
L_{\lambda}^{\ddot{w} \sharp} \approx\left\{\left(L_{\lambda}^{\left(z \eta^{\prime}\right)}\right)_{h}\langle-i\rangle ; \eta^{\prime} \in W_{\lambda}, h \in\left[1, n_{\lambda, \eta^{\prime}, \eta, i}\right]\right\} .
$$


This is compatible with the natural mixed structures. Using 2.9(a), we deduce

$$
\gamma\left(L_{\lambda}^{\ddot{w} \sharp}\right)=\sum_{\eta^{\prime} \in W_{\lambda} ; i \in 2 \mathbf{Z}} n_{\lambda, \eta^{\prime}, \eta, i} \gamma\left(L_{\lambda}^{\left(z \eta^{\prime}\right)}\right) v^{i},
$$

that is

$$
\gamma\left(L_{\lambda}^{\ddot{w} \sharp}\right)=\sum_{\eta^{\prime} \in W_{\lambda}} v^{|w|-\left|z \eta^{\prime}\right|-|\eta|_{\lambda}+\left|\eta^{\prime}\right|_{\lambda}} P_{\eta^{\prime}, \eta}^{\lambda}\left(v^{2}\right) T_{z \eta^{\prime}} 1_{\lambda}
$$

hence, using 1.8(a),

$$
\gamma\left(L_{\lambda}^{\omega \sharp}\right)=v^{|w|} c_{w, \lambda},
$$

for any $\omega \in \kappa_{q}^{-1}(w)$.

2.13. Let $w, w^{\prime} \in W, \omega \in \kappa^{-1}(w), \omega^{\prime} \in \kappa^{-1}\left(w^{\prime}\right)$ and $\lambda, \lambda^{\prime} \in \mathfrak{s}$. We show:

(a) If $w^{\prime}\left(\lambda^{\prime}\right) \neq \lambda$ then $L_{\lambda}^{\omega \sharp} \circ L_{\lambda^{\prime}}^{\omega^{\prime} \sharp}=0$.

(b) If $w^{\prime}\left(\lambda^{\prime}\right) \neq \lambda$ then $L_{\lambda}^{\omega} \circ L_{\lambda^{\prime}}^{\omega^{\prime} \sharp}=0, L_{\lambda}^{\omega \sharp} \circ L_{\lambda^{\prime}}^{\omega^{\prime}}=0$.

We prove (a). We write $w=z w_{1}\left(\operatorname{resp} . w^{\prime}=z^{\prime} w_{1}^{\prime}\right)$ where $z=\min \left(z W_{\lambda}\right)$ (resp. $z^{\prime}=\min \left(z^{\prime} W_{\lambda^{\prime}}\right)$ ) and $w_{1} \in W_{\lambda}$ (resp. $\left.w_{1}^{\prime} \in W_{\lambda^{\prime}}\right)$. Using 2.12(c) it is enough to show that $L_{\lambda}^{\dot{y}_{1}} \circ L_{\lambda^{\prime}}^{\dot{y}_{1}^{\prime}}=0$ for any $y_{1} \in W_{\lambda}, y_{1}^{\prime} \in W_{\lambda^{\prime}}$. Using 2.3 (a) it is enough to show that for $y_{1}^{\prime} \in W_{\lambda^{\prime}}$ we have $z^{\prime} y_{1}^{\prime}\left(\lambda^{\prime}\right) \neq \lambda$. We have $y_{1}^{\prime}\left(\lambda^{\prime}\right)=\lambda^{\prime}, w_{1}^{\prime}\left(\lambda^{\prime}\right)=\lambda$ hence $z^{\prime} y_{1}^{\prime}\left(\lambda^{\prime}\right)=z^{\prime}\left(\lambda^{\prime}\right)=z^{\prime} w_{1}^{\prime}\left(\lambda^{\prime}\right)=w^{\prime}\left(\lambda^{\prime}\right)$. It remains to use our assumption that $w^{\prime}\left(\lambda^{\prime}\right) \neq \lambda$.

We prove (b). For the first (resp. second) equality in (b) we repeat the proof of (a) but take $y_{1}=w_{1}$ (resp. $\left.y_{1}^{\prime}=w_{1}^{\prime}\right)$.

It is not difficult to prove the following strengthening of (a).

(c) Assume that for some $j \in \mathbf{Z}, \mathbf{L}_{\eta}^{\dot{z}}$ is a composition factor of $\left(\mathbf{L}_{\lambda}^{\dot{w}} \circ \mathbf{L}_{\lambda^{\prime}}^{\dot{w}^{\prime}}\right)^{j}$. Then $\eta=\lambda^{\prime}=w^{\prime-1}(\lambda)$.

2.14. In the remainder of this paper we fix a two-sided cell $\mathbf{c}$ of $W \mathfrak{s}$ and we set $a=a(w \cdot \lambda)$ for some/any $w \cdot \lambda \in \mathbf{c}$. Let $\mathfrak{o}$ be the unique $W$-orbit on $\mathfrak{s}$ such that $w \cdot \lambda \in \mathbf{c} \Longrightarrow \lambda \in \mathfrak{o}$.

Let $Y=\tilde{\mathcal{B}}^{2}$. Let $\mathcal{M} \preceq Y$ (resp. $\mathcal{M}^{\prec} Y$ ) be the subcategory of $\mathcal{D}^{\wedge} Y$ whose objects are perverse sheaves $L$ such that any composition factor of $L$ is of 
the form $\mathbf{L}_{\lambda}^{\dot{w}}$ for some $w \cdot \lambda \preceq \mathbf{c}($ resp. $w \cdot \lambda \prec \mathbf{c})$. Let $\mathcal{M}_{m}^{\prec} Y$ (resp. $\mathcal{M}_{m}^{\prec} Y$ ) be the subcategory of $\mathcal{D}_{m}^{\wedge} Y$ whose objects are in $\mathcal{M}^{\checkmark} Y$ (resp. $\mathcal{M}^{\prec} Y$ ). Let $\mathcal{D} \preceq Y$ (resp. $\mathcal{D}^{\prec} Y$ ) be the subcategory of $\mathcal{D}^{\natural} Y$ whose objects are complexes $L$ such that $L^{j}$ is in $\mathcal{M} \preceq Y$ (resp. $\mathcal{M}^{\prec} Y$ ) for any $j$. Let $\mathcal{D}_{\bar{m}}^{\prec} Y$ (resp. $\mathcal{D}_{m}^{\prec} Y$ ) be the subcategory of $\mathcal{D}_{m}^{\mathfrak{\phi}} Y$ whose objects are also in $\mathcal{D}^{\preceq} Y$ (resp. $\mathcal{D}^{\prec} Y$ ). Let $\mathcal{C} Y$ be the subcategory of $\mathcal{M} Y$ consisting of semisimple objects. Let $\mathcal{C}_{0}^{\wedge} Y$ be the subcategory of $\mathcal{M}_{m}^{\uparrow} Y$ consisting of those $L$ such that $L$ is pure of weight zero. Let $\mathcal{C}^{\mathbf{c}} Y$ be the subcategory of $\mathcal{M}^{\top} Y$ consisting of objects which are direct sums of objects of the form $\mathbf{L}_{\lambda}^{\dot{w}}$ with $w \cdot \lambda \in \mathbf{c}$. Let $\mathcal{C}_{0}^{\mathbf{c}} Y$ be the subcategory of $\mathcal{C}_{0}^{\top} Y$ consisting of those $L \in \mathcal{C}_{0}^{\uparrow} Y$ such that, as an object of $\mathcal{C}^{\uparrow} Y, L$ belongs to $\mathcal{C}^{\mathbf{c}} Y$. For $L \in \mathcal{C}_{0}^{\boldsymbol{m}} Y$ let $\underline{L}$ be the largest subobject of $L$ such that as an object of $\mathcal{C}^{\top} Y$, we have $\underline{L} \in \mathcal{C}^{\mathbf{c}} Y$.

2.15. Let $r \geq 1$. We define an action of $\mathcal{G}=G \times \mathbf{U}^{r} \times \mathbf{T}^{2 r+1} \times \mathbf{U}^{r+1}$ on $G^{r+1}$ by

$$
\begin{gathered}
\left(g, u_{1}, u_{2}, \ldots, u_{r}, t_{1}, \ldots, t_{r}, t_{0}^{\prime}, t_{1}^{\prime}, \ldots, t_{r}^{\prime}, u_{0}^{\prime}, u_{1}^{\prime}, \ldots, u_{r}^{\prime}\right): \\
\left(g_{0}, g_{1}, \ldots, g_{r}\right) \mapsto\left(g g_{0} t_{0}^{\prime n} u_{0}^{\prime-1}, u_{1} t_{1}^{-n} g_{1} t_{1}^{\prime n} u_{1}^{\prime-1}, \ldots, u_{r} t_{r}^{-n} g_{r} t_{r}^{\prime n} u_{r}^{\prime-1}\right) .
\end{gathered}
$$

The orbits of this action are indexed by $W^{r}$; to $\mathbf{w}=\left(w_{1}, \ldots, w_{r}\right) \in W^{r}$ corresponds the orbit $G_{\mathbf{w}}^{r+1}=G \times G_{w_{1}} \times G_{w_{2}} \times \ldots \times G_{w_{r}}$. The restriction of the $\mathcal{G}$-action to the subgroup

$$
\begin{aligned}
\mathcal{G}^{\prime}:= & \left\{\left(g, u_{1}, u_{2}, \ldots, u_{r}, t_{1}, \ldots, t_{r}, t_{0}^{\prime}, t_{1}^{\prime}, \ldots, t_{r}^{\prime}, u_{0}^{\prime}, u_{1}^{\prime}, \ldots, u_{r}^{\prime}\right) \in \mathcal{G} ;\right. \\
& \left.g=1, t_{1}=\ldots=t_{r}=t_{0}^{\prime}=\ldots=t_{r}^{\prime}=1, u_{0}^{\prime}=u_{1}, u_{1}^{\prime}=u_{2}, \ldots, u_{r-1}^{\prime}=u_{r}\right\}
\end{aligned}
$$

(isomorphic to $\mathbf{U}^{r+1}$ ) is free and the map $\theta: G^{r+1} \rightarrow \tilde{\mathcal{B}}^{r+1}$ given by

$$
\left(g_{0}, g_{1}, \ldots, g_{r}\right) \mapsto\left(g_{0} \mathbf{U}, g_{0} g_{1} \mathbf{U}, \ldots, g_{0} g_{1} \ldots g_{r} \mathbf{U}\right)
$$

identifies $\tilde{\mathcal{B}}^{r+1}$ with $\mathcal{G}^{\prime} \backslash G^{r+1}$. For $\mathbf{w}=\left(w_{1}, \ldots, w_{r}\right) \in W^{r}$ and $J \subset[1, r]$ we define

$$
\begin{aligned}
G_{\mathbf{w}}^{r+1, J}= & \left\{\left(g_{0}, g_{1}, \ldots, g_{r}\right) \in G^{r+1} ; g_{i} \in \bar{G}_{w_{i}} \forall i \in J, g_{i} \in G_{w_{i}} \forall i \in[1, r]-J\right\}, \\
\tilde{\mathcal{O}}_{\mathbf{w}}^{J}= & \left\{\left(x_{0} \mathbf{U}, x_{1} \mathbf{U}, \ldots, x_{r} \mathbf{U}\right) \in \tilde{\mathcal{B}}^{r+1} ; x_{i-1}^{-1} x_{i} \mathbf{U} \in \bar{G}_{w_{i}} \forall i \in J,\right. \\
& \left.x_{i-1}^{-1} x_{i} \in G_{w_{i}} \forall i \in[1, r]-J\right\} .
\end{aligned}
$$


Now $\theta$ identifies $\tilde{\mathcal{O}}_{\mathbf{w}}^{J}$ with $\mathcal{G}^{\prime} \backslash G_{\mathbf{w}}^{r+1, J}$ and

$$
\tilde{\mathcal{O}}_{\mathbf{w}}^{J}=\sqcup_{\mathbf{y}=\left(y_{1}, y_{2}, \ldots, y_{r}\right) \in W^{r} ; y_{i} \leq w_{i} \forall i \in J, y_{i}=w_{i} \forall i \in[1, r]-J} \tilde{\mathcal{O}}_{\mathbf{y}}^{\emptyset} .
$$

Note that $\tilde{\mathcal{O}}_{\mathbf{w}}^{\emptyset}$ is irreducible of dimension $\nu+(r+1) \rho+|\mathbf{w}|$ where

$$
|\mathbf{w}|=\left|w_{1}\right|+\left|w_{2}\right|+\cdots+\left|w_{r}\right| .
$$

Until the end of 2.22 we fix $\mathbf{w}=\left(w_{1}, \ldots, w_{r}\right) \in W^{r}, \boldsymbol{\omega}=\left(\omega_{1}, \omega_{2}, \ldots, \omega_{r}\right)$ such that $\omega_{i} \in \kappa_{q}^{-1}\left(w_{i}\right)$ for $i=1, \ldots, r$ and $\boldsymbol{\lambda}=\left(\lambda_{1}, \lambda_{2}, \ldots, \lambda_{r}\right) \in \mathfrak{s}^{r}$.

Define $c: \tilde{\mathcal{O}}_{\mathbf{w}}^{\emptyset} \rightarrow \mathbf{T}^{r}$ and $\tilde{c}: G_{\mathbf{w}}^{r+1} \rightarrow \mathbf{T}^{r}$ by

$$
\begin{aligned}
c\left(x_{0} \mathbf{U}, x_{1} \mathbf{U}, \ldots, x_{r} \mathbf{U}\right) & =\left(\left(x_{0}^{-1} x_{1}\right)_{\omega_{1}},\left(x_{1}^{-1} x_{2}\right)_{\omega_{2}}, \ldots,\left(x_{r-1}^{-1} x_{r}\right)_{\omega_{r}}\right), \\
\tilde{c}\left(g_{0}, g_{1}, \ldots, g_{r}\right) & =\left(\left(g_{1}\right)_{\omega_{1}},\left(g_{2}\right)_{\omega_{2}}, \ldots,\left(g_{r}\right)_{\omega_{r}}\right),
\end{aligned}
$$

so that $\tilde{c}=c \theta$. Let $M_{\lambda}^{\omega} \in \mathcal{D}_{m}\left(\tilde{\mathcal{B}}^{r+1}\right)$ be the local system $c^{*}\left(L_{\lambda_{1}} \otimes \ldots \otimes L_{\lambda_{r}}\right)$ on $\tilde{\mathcal{O}}_{\mathbf{w}}^{\emptyset}$ extended by 0 on $\tilde{\mathcal{B}}^{r+1}-\tilde{\mathcal{O}}_{\mathbf{w}}^{\emptyset}$. Let $\tilde{M}_{\lambda}^{\omega} \in \mathcal{D}_{m}\left(G^{r+1}\right)$ be the local system $\tilde{c}^{*}\left(L_{\lambda_{1}} \otimes \ldots \otimes L_{\lambda_{r}}\right)$ on $G_{\mathbf{w}}^{r+1}$ extended by 0 on $G^{r+1}-G_{\mathbf{w}}^{r+1}$. Note that

$$
\tilde{M}_{\lambda}^{\omega}=\theta^{*} M_{\lambda}^{\omega}
$$

From the definitions we have

$$
M_{\lambda}^{\omega}=p_{01}^{*} L_{\lambda_{1}}^{\omega_{1}} \otimes p_{12}^{*} L_{\lambda_{2}}^{\omega_{2}} \otimes \ldots \otimes p_{r-1, r}^{*} L_{\lambda_{r}}^{\omega_{r}}
$$

(Here $p_{i j}: \tilde{\mathcal{B}}^{r+1} \rightarrow \tilde{\mathcal{B}}^{2}$ are the obvious projections.) Note that $\tilde{M}_{\boldsymbol{\lambda}}^{\omega} \in$ $\mathcal{D}_{m}\left(G^{r+1}\right)$ is $\mathcal{G}$-equivariant. Indeed, $\mathcal{G}$ acts on $\mathbf{T}^{r}$ by

$$
\begin{aligned}
& \left(g, u_{1}, u_{2}, \ldots, u_{r}, t_{1}, \ldots, t_{r}, t_{0}^{\prime}, t_{1}^{\prime}, \ldots, t_{r}^{\prime}, u_{0}^{\prime}, u_{1}^{\prime}, \ldots, u_{r}^{\prime}\right): \\
& \quad\left(t_{1}^{\prime \prime}, t_{2}^{\prime \prime}, \ldots, t_{r}^{\prime \prime}\right) \mapsto\left(w_{1}^{-1}\left(t_{1}^{-n}\right) t_{1}^{\prime \prime} t_{1}^{\prime n}, w_{2}^{-1}\left(t_{2}^{-n}\right) t_{2}^{\prime \prime} t_{2}^{\prime n}, \ldots, w_{r}^{-1}\left(t_{r}^{-n}\right) t_{r}^{\prime \prime} t_{r}^{\prime n}\right),
\end{aligned}
$$

$\theta$ is compatible with the $\mathcal{G}$-actions and $L_{\lambda_{1}} \otimes \ldots \otimes L_{\lambda_{r}}$ is a $\mathcal{G}$-equivariant local system. Let $J \subset[1, r]$. We set

$$
\begin{aligned}
M_{\boldsymbol{\lambda}}^{\boldsymbol{\omega}, J} & =p_{01}^{*} L \otimes p_{12}^{*} L \otimes \ldots \otimes p_{r-1, r}^{*} L \in \mathcal{D}_{m}\left(\tilde{\mathcal{B}}^{r+1}\right), \\
L_{\boldsymbol{\lambda}}^{\boldsymbol{\omega}, J} & =p_{0 r !} M_{\boldsymbol{\lambda}}^{\boldsymbol{\omega}, J}\langle|\mathbf{w}|\rangle={ }^{1} L \circ{ }^{2} L \circ \ldots \circ{ }^{r} L\langle|\mathbf{w}|\rangle \in \mathcal{D}_{m}\left(\tilde{\mathcal{B}}^{2}\right),
\end{aligned}
$$


where ${ }^{i} L$ is $L_{\lambda_{i}}^{\omega_{i} \sharp}$ for $i \in J$ and $L_{\lambda_{i}}^{\omega_{i}}$ for $i \notin J$. Note that $M_{\lambda}^{\omega, \emptyset}=M_{\lambda}^{\omega}$. Moreover, $M_{\lambda}^{\omega, J}$ is the intersection cohomology complex of $\tilde{\mathcal{O}}_{\mathbf{w}}^{J}$ with coefficients in $M_{\lambda}^{\omega}$.

To prove this, it is enough to show that $\theta^{*} M_{\lambda}^{\omega, J}$ is the intersection cohomology complex of $G_{\mathbf{w}}^{r+1, J}$ with coefficients in $\tilde{M}_{\lambda}^{\omega}$; this is immediate.

Consider the free $\mathbf{T}^{r-1}$-action on $\tilde{\mathcal{B}}^{r+1}$ given by

$$
\begin{aligned}
& \left(\tau_{1}, \tau_{2}, \ldots, \tau_{r-1}\right):\left(x_{0} \mathbf{U}, x_{1} \mathbf{U}, \ldots, x_{r-1} \mathbf{U}, x_{r} \mathbf{U}\right) \mapsto \\
& \left(x_{0} \mathbf{U}, x_{1} \tau_{1} \mathbf{U}, \ldots, x_{r-1} \tau_{r-1} \mathbf{U}, x_{r} \mathbf{U}\right) .
\end{aligned}
$$

Note that $\tilde{\mathcal{O}}_{\mathbf{w}}^{J}$ is stable under this $\mathbf{T}^{r-1}$-action. We also have a free $\mathbf{T}^{r-1}$ action on $\mathbf{T}^{r}$ given by

$$
\begin{gathered}
\left(\tau_{1}, \tau_{2}, \ldots, \tau_{r-1}\right):\left(t_{1}, t_{2}, \ldots, t_{r}\right) \mapsto \\
\left(t_{1} \tau_{1}, w_{2}^{-1}\left(\tau_{1}^{-1}\right) t_{2} \tau_{2}, w_{3}^{-1}\left(\tau_{2}^{-1}\right) t_{3} \tau_{3}, \ldots, w_{r-1}^{-1}\left(\tau_{r-2}^{-1}\right) t_{r-1} \tau_{r-1}, w_{r}^{-1}\left(\tau_{r-1}^{-1}\right) t_{r}\right) .
\end{gathered}
$$

Let ${ }^{\prime} \tilde{\mathcal{B}}^{r+1}=\mathbf{T}^{r-1} \backslash \tilde{\mathcal{B}}^{r+1}$. Let ${ }^{\prime} \tilde{\mathcal{O}}_{\mathbf{w}}^{J}=\mathbf{T}^{r-1} \backslash \tilde{\mathcal{O}}_{\mathbf{w}}^{J}$ (a locally closed subvariety of $\left.{ }^{\prime} \tilde{\mathcal{B}}^{r+1}\right)$. Let ${ }^{\prime} \mathbf{T}^{r}=\mathbf{T}^{r-1} \backslash \mathbf{T}^{r}$. Note that ${ }^{\prime} \tilde{\mathcal{O}}_{\mathbf{w}}^{\emptyset}=\mathbf{T}^{r-1} \backslash \tilde{\mathcal{O}}_{\mathbf{w}}^{\emptyset}$ is an open dense smooth irreducible subvariety of ${ }^{\prime} \tilde{\mathcal{O}}_{\mathbf{w}}^{J}$. Now $c: \tilde{\mathcal{O}}_{\mathbf{w}}^{\emptyset} \rightarrow \mathbf{T}^{r}$ is compatible with the $\mathbf{T}^{r-1}$-actions on $\tilde{\mathcal{O}}_{\mathbf{w}}^{\emptyset}, \mathbf{T}^{r}$ hence it induces a map ${ }^{\prime} c:{ }^{\prime} \tilde{\mathcal{O}}_{\mathbf{w}}^{\emptyset} \rightarrow{ }^{\prime} \mathbf{T}^{r}$. The homomorphism $c^{\prime}: \mathbf{T}^{r} \rightarrow \mathbf{T}$ given by

$$
\left(t_{1}, t_{2}, \ldots, t_{r}\right) \mapsto t_{1} w_{2}\left(t_{2}\right) w_{2} w_{3}\left(t_{3}\right) \ldots w_{2} w_{3} \ldots w_{r}\left(t_{r}\right)
$$

is constant on each orbit of the $\mathbf{T}^{r-1}$-action on $\mathbf{T}^{r}$ hence it induces a morphism ${ }^{\prime} \mathbf{T}^{r} \rightarrow \mathbf{T}$ whose composition with ${ }^{\prime} c$ is denoted by $\bar{c}:{ }^{\prime} \tilde{\mathcal{O}}_{\mathbf{w}}^{\emptyset} \rightarrow \mathbf{T}$. Let ${ }^{\prime} M_{\lambda}^{\omega, \emptyset}$ be the local system $\bar{c}^{*} L_{\lambda_{1}}$ on ${ }^{\prime} \tilde{\mathcal{O}}_{\mathbf{w}}^{\emptyset}$ extended by 0 on ${ }^{\prime} \tilde{\mathcal{B}}^{r+1}-{ }^{\prime} \tilde{\mathcal{O}}_{\mathbf{w}}^{\emptyset}$. Let ${ }^{\prime} M_{\lambda}^{\omega, J} \in \mathcal{D}_{m}\left({ }^{\prime} \tilde{\mathcal{B}}^{r+1}\right)$ be the intersection cohomology complex of ' $\tilde{\mathcal{O}}_{\mathbf{w}}^{J}$ with coefficients in ${ }^{\prime} M_{\boldsymbol{\lambda}}^{\boldsymbol{\omega}, \emptyset}$ extended by 0 on ${ }^{\prime} \tilde{\mathcal{B}}^{r+1}-{ }^{\prime} \tilde{\mathcal{O}}_{\mathbf{w}}^{J}$. Let $\bar{p}_{0 r}:{ }^{\prime} \tilde{\mathcal{O}}_{\mathbf{w}}^{J} \rightarrow \tilde{\mathcal{B}}^{2}$ be the map induced by $p_{0 r}: \tilde{\mathcal{O}}_{\mathbf{w}}^{J} \rightarrow \tilde{\mathcal{B}}^{2}$. We define ${ }^{\prime} L_{\boldsymbol{\lambda}}^{\boldsymbol{\omega}, J} \in \mathcal{D}_{m}^{\mathbf{\phi}} \tilde{\mathcal{B}}^{2}$ as follows: if

$$
\lambda_{k}=w_{k+1}\left(\lambda_{k+1}\right) \text { for } k=1,2, \ldots, r-1
$$

(in which case we say that $\boldsymbol{\lambda}$ is $\mathbf{w}$-adapted) we set

$$
{ }^{\prime} L_{\boldsymbol{\lambda}}^{\boldsymbol{\omega}, J}=\bar{p}_{0 r !}{ }^{\prime} M_{\boldsymbol{\lambda}}^{\boldsymbol{\omega}, J}\langle|\mathbf{w}|\rangle ;
$$

if $\boldsymbol{\lambda}$ is not $\mathbf{w}$-adapted, we set ${ }^{\prime} L_{\boldsymbol{\lambda}}^{\boldsymbol{\omega}, J}=0$. Let $\hat{c}^{J}: \tilde{\mathcal{O}}_{\mathbf{w}}^{J} \rightarrow{ }^{\prime} \tilde{\mathcal{O}}_{\mathbf{w}}^{J}$ be the obvious (orbit) map. We show: 
(a) If $\boldsymbol{\lambda}$ is $\mathbf{w}$-adapted then $M_{\boldsymbol{\lambda}}^{\boldsymbol{\omega}, J}=\left(\hat{c}^{J}\right)^{* \prime} M_{\boldsymbol{\lambda}}^{\boldsymbol{\omega}, J}$.

Since $\hat{c}^{J}$ is a $\mathbf{T}^{r-1}$-bundle, it is enough to show that

$$
M_{\lambda}^{\omega, \emptyset}=\left(\hat{c}^{\emptyset}\right)^{* \prime} M_{\lambda}^{\omega, \emptyset}
$$

or that

$$
c^{*}\left(L_{\lambda_{1}} \otimes \ldots \otimes L_{\lambda_{r}}\right)=\left(\hat{c}^{\emptyset}\right)^{*} \bar{c}^{*} L_{\lambda_{1}} .
$$

We have a commutative diagram

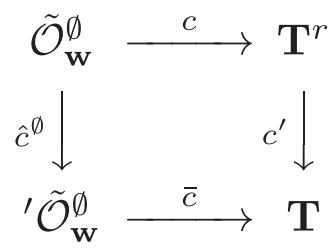

hence $\left(\hat{c}^{\emptyset}\right)^{*} \bar{c}^{*}=c^{*} c^{*}$ and it is enough to show that

$$
L_{\lambda_{1}} \otimes \ldots \otimes L_{\lambda_{r}}=c^{* *} L_{\lambda_{1}}
$$

This follows from the equality

$$
\lambda_{1}\left(c^{\prime}\left(t_{1}, t_{2}, \ldots, t_{r}\right)\right)=\lambda_{1}\left(t_{1}\right) \lambda_{2}\left(t_{2}\right) \ldots \lambda_{r}\left(t_{r}\right) \text { for all }\left(t_{1}, t_{2}, \ldots, t_{r}\right) \in \mathbf{T}_{n}^{r}
$$

which is a consequence of $\boldsymbol{\lambda}$ being $\mathbf{w}$-adapted.

We now show:

(b) We have $L_{\boldsymbol{\lambda}}^{\boldsymbol{\omega}, J}=\mathfrak{L}^{\otimes(r-1)} \otimes^{\prime} L_{\boldsymbol{\lambda}}^{\boldsymbol{\omega}, J}$.

If $\boldsymbol{\lambda}$ is not w-adapted then from 2.3(a), 2.13(a), (b), we see that $L_{\boldsymbol{\lambda}}^{\boldsymbol{\omega}, J}=0$ hence (b) holds. We now assume that $\boldsymbol{\lambda}$ is $\mathbf{w}$-adapted. Using (a) we have

$$
\begin{aligned}
L_{\boldsymbol{\lambda}}^{\boldsymbol{\omega}, J} & =p_{0 r !} M_{\boldsymbol{\lambda}}^{\boldsymbol{\omega}, J}\langle|\mathbf{w}|\rangle=p_{0 r !}\left(\hat{c}^{J}\right)^{* \prime} M_{\boldsymbol{\lambda}}^{\boldsymbol{\omega}, J}\langle|\mathbf{w}|\rangle \\
& \left.=\bar{p}_{0 r !}\left(\hat{c}^{J}\right)_{!}\left(\hat{c}^{J}\right)^{* \prime} M_{\boldsymbol{\lambda}}^{\boldsymbol{\omega}, J}\langle|\mathbf{w}|\rangle=\bar{p}_{0 r !}\left(\left(\hat{c}^{J}\right) ! \overline{\mathbf{Q}}_{l}\right) \otimes\left(\hat{c}^{J}\right)^{* \prime} M_{\boldsymbol{\lambda}}^{\boldsymbol{\omega}, J}\langle|\mathbf{w}|\rangle\right)
\end{aligned}
$$

and it remains to use that $\left(\hat{c}^{J}\right) ! \overline{\mathbf{Q}}_{l}=\mathfrak{L}^{\otimes(r-1)}$.

We prove the following result.

(c) There is a natural bijection between $\mathfrak{s}^{r}$ and the set of isomorphism classes of irreducible $\mathcal{G}$-equivariant local systems on the $\mathcal{G}$-orbit $G_{\mathrm{w}}^{r+1}$ : to $\boldsymbol{\lambda}^{\prime}=$ 
$\left(\lambda_{1}^{\prime}, \lambda_{2}^{\prime}, \ldots, \lambda_{r}^{\prime}\right) \in \mathfrak{s}^{r}$ corresponds the local system

$\left.\tilde{M}_{\boldsymbol{\lambda}^{\prime}}^{\omega^{\prime}}\right|_{G_{\mathbf{w}^{\prime}}}=\left(\theta^{*} M_{\boldsymbol{\lambda}^{\prime}}^{\omega^{\prime}}\right)_{G_{\mathbf{w}^{\prime}}}$ where $\boldsymbol{\omega}^{\prime}=\left(\dot{w}_{1}, \dot{w}_{2}, \ldots, \dot{w}_{r}\right)$.

Let $\Gamma$ be the stabilizer of $\left(1, \dot{w}_{1}, \ldots, \dot{w}_{r}\right) \in G_{\mathbf{w}}^{r+1}$ in $\mathcal{G}$. We have

$$
\begin{aligned}
\Gamma= & \left\{\left(g, u_{1}, u_{2}, \ldots, u_{r}, t_{1}, \ldots, t_{r}, t_{0}^{\prime}, t_{1}^{\prime}, \ldots, t_{r}^{\prime}, u_{0}^{\prime}, u_{1}^{\prime}, \ldots, u_{r}^{\prime}\right) \in \mathcal{G} ;\right. \\
& g=u_{0}^{\prime} t_{0}^{\prime-n}, \dot{w}_{1}^{-1} u_{1} \dot{w}_{1}=u_{1}^{\prime}, \dot{w}_{2}^{-1} u_{2} \dot{w}_{2}=u_{2}^{\prime}, \ldots, \dot{w}_{r}^{-1} u_{r} \dot{w}_{r}=u_{r}^{\prime}, \\
& \left.t_{1}^{n}=w_{1}\left(t_{1}^{\prime n}\right), \ldots, t_{r}^{n}=w_{r}\left(t_{r}^{\prime n}\right) .\right\}
\end{aligned}
$$

The closed subgroup

$$
\begin{aligned}
& \left\{\left(g, u_{1}, u_{2}, \ldots, u_{r}, t_{1}, \ldots, t_{r}, t_{0}^{\prime}, t_{1}^{\prime}, \ldots, t_{r}^{\prime}, u_{0}^{\prime}, u_{1}^{\prime}, \ldots, u_{r}^{\prime}\right) \in \mathcal{G}\right. \\
& \quad g=u_{0}^{\prime} t_{0}^{\prime-n}, \dot{w}_{1}^{-1} u_{1} \dot{w}_{1}=u_{1}^{\prime}, \dot{w}_{2}^{-1} u_{2} \dot{w}_{2}=u_{2}^{\prime}, \ldots, \dot{w}_{r}^{-1} u_{r} \dot{w}_{r}=u_{r}^{\prime} \\
& \left.\quad t_{1}=w_{1}\left(t_{1}^{\prime}\right), \ldots, t_{r}=w_{r}\left(t_{r}^{\prime}\right)\right\}
\end{aligned}
$$

of $\Gamma$ is clearly connected of the same dimension as $\Gamma$ (namely $(r+1) \nu+(r+$ 1) $\rho$ ) hence it is equal to the identity component $\Gamma^{0}$ of $\Gamma$. We can view $\mathbf{T}_{n}^{2 r}$ as the kernel of the surjective homomorphism $\Gamma \rightarrow G \times \mathbf{U}^{r} \times \mathbf{T}^{r+1} \times \mathbf{U}^{r+1}$,

$$
\begin{aligned}
& \left(g, u_{1}, u_{2}, \ldots, u_{r}, t_{1}, \ldots, t_{r}, t_{0}^{\prime}, t_{1}^{\prime}, \ldots, t_{r}^{\prime}, u_{0}^{\prime}, u_{1}^{\prime}, \ldots, u_{r}^{\prime}\right) \\
& \mapsto\left(u_{1}, u_{2}, \ldots, u_{r}, t_{1}^{n}, \ldots, t_{r}^{n}, t_{0}^{\prime}, u_{0}^{\prime}, u_{1}^{\prime}, \ldots, u_{r}^{\prime}\right)
\end{aligned}
$$

whose restriction to $\Gamma^{0}$ must also be surjective. It follows that $\Gamma=\mathbf{T}_{n}^{2 r} \Gamma^{0}$ hence

$$
\begin{aligned}
\Gamma / \Gamma^{0} & =\mathbf{T}_{n}^{2 r} /\left(\mathbf{T}_{n}^{2 r} \cap \Gamma^{0}\right) \\
& =\mathbf{T}_{n}^{2 r} /\left\{\left(t_{1}, \ldots, t_{r}, t_{1}^{\prime}, \ldots, t_{r}^{\prime}\right) \in \mathbf{T}_{n}^{2 r} ; t_{1}=w_{1}\left(t_{1}^{\prime}\right), \ldots, t_{r}=w_{r}\left(t_{r}^{\prime}\right)\right\}
\end{aligned}
$$

Note that the irreducible $\mathcal{G}$-equivariant local systems on $G_{\mathrm{w}}^{r+1}$ correspond to irreducible representations of $\Gamma / \Gamma^{0}$ hence to representations of $\mathbf{T}_{n}^{2 r}$ which are trivial on

$$
\left\{\left(t_{1}, \ldots, t_{r}, t_{1}^{\prime}, \ldots, t_{r}^{\prime}\right) \in T_{n}^{2 r} ; t_{1}=w_{1}\left(t_{1}^{\prime}\right), \ldots, t_{r}=w_{r}\left(t_{r}^{\prime}\right)\right\}
$$

Such representations are uniquely determined by their restriction to

$$
\left\{\left(t_{1}, \ldots, t_{r}, t_{1}^{\prime}, \ldots, t_{r}^{\prime}\right) \in \mathbf{T}_{n}^{2 r} ; t_{1}=t_{2}=\cdots=t_{r}=1\right\}
$$


hence they are in natural bijection with $\mathfrak{s}^{r}$. This proves $(\mathrm{c})$.

Using (c) and the fact that the $\mathcal{G}$-orbits on $G^{r+1}$ are indexed by $W^{r}$, we deduce:

(d) There is a natural bijection between $W^{r} \times \mathfrak{s}^{r}$ and the set of isomorphism classes of simple $\mathcal{G}$-equivariant perverse sheaves on $G^{r+1}:$ to $\mathbf{w}^{\prime}=$ $\left(w_{1}^{\prime}, w_{2}^{\prime}, \ldots, w_{r}^{\prime}\right) \in W^{r}$ and $\boldsymbol{\lambda}^{\prime}=\left(\lambda_{1}^{\prime}, \lambda_{2}^{\prime}, \ldots, \lambda_{r}^{\prime}\right) \in \mathfrak{s}^{r}$ corresponds the simple perverse sheaf $\theta^{*} M_{\boldsymbol{\lambda}^{\prime}}^{\boldsymbol{\omega}^{\prime},[1, r]}\left\langle\operatorname{dim} G_{\mathbf{w}^{\prime}}^{r+1}\right\rangle$ where $\boldsymbol{\omega}^{\prime}=\left(\dot{w}_{1}^{\prime}, \dot{w}_{2}^{\prime}, \ldots, \dot{w}_{r}^{\prime}\right)$.

2.16. We preserve the setup of 2.15 . We assume that $J=[1, r]$. In this case, $\bar{p}_{0 r}:{ }^{\prime} \tilde{\mathcal{O}}_{\mathbf{w}}^{[1, r]} \rightarrow \tilde{\mathcal{B}}^{2}$ is clearly a proper morphism. Hence, by Deligne's theorem,

(a) ' $L_{\boldsymbol{\lambda}}^{\boldsymbol{\omega},[1, r]}$ is pure of weight zero.

We set $L=L_{\boldsymbol{\lambda}}^{\boldsymbol{\omega},[1, r]},{ }^{\prime} L={ }^{\prime} L_{\boldsymbol{\lambda}}^{\boldsymbol{\omega},[1, r]}$. From (a) it follows that for $j \in \mathbf{Z},{ }^{\prime} L^{j}$ is pure of weight $j$ hence

$$
{ }^{\prime} L^{j}=\oplus_{w \cdot \lambda \in W \mathfrak{s}} \bar{V}_{w \cdot \lambda, j} \mathbf{L}_{\lambda}^{\dot{w}}
$$

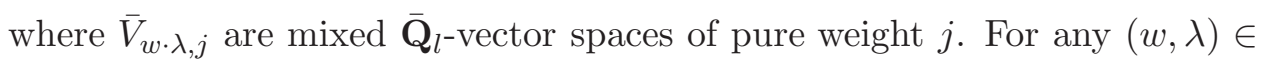
$W \mathfrak{s}$ and any $j \in \mathbf{Z}$ we show:

(c) We have

$$
\operatorname{dim} \bar{V}_{w \cdot \lambda, j}=N(w \cdot \lambda,-j+\nu+2 \rho)
$$

where $N(w, \lambda, k)=N(w, \lambda,-k) \in \mathbf{N}$ are given by the equality $($ in $\mathbf{H})$ :

$$
c_{w_{1} \cdot \lambda_{1}} c_{w_{2} \cdot \lambda_{2}} \ldots c_{w_{r} \cdot \lambda_{r}}=\sum_{w \cdot \lambda \in W \mathfrak{s}, k \in \mathbf{Z}} N(w \cdot \lambda, k) v^{k} c_{w \cdot \lambda} .
$$

From 2.11(a) and 2.12(d) we have (setting $\delta=(r-1) \rho)$ :

$$
\begin{aligned}
\gamma(L) & =\left(v^{2}-1\right)^{\delta} \gamma\left(L_{\lambda_{1}}^{\omega_{1}}\right) \gamma\left(L_{\lambda_{2}}^{\omega_{2} \sharp}\right) \ldots \gamma\left(L_{\lambda_{r}}^{\omega_{r}}\right) v^{-|\mathbf{w}|} \\
& =\left(v^{2}-1\right)^{\delta} v^{\left|w_{1}\right|} c_{w_{1} \cdot \lambda_{1}} v^{\left|w_{2}\right|} c_{w_{2} \cdot \lambda_{2}} \ldots v^{\left|w_{r}\right|} c_{w_{r} \cdot \lambda_{r}} v^{-|\mathbf{w}|} \\
& =\left(v^{2}-1\right)^{\delta} c_{w_{1} \cdot \lambda_{1}} c_{w_{2} \cdot \lambda_{2}} \ldots c_{w_{r} \cdot \lambda_{r}} \\
& =\left(v^{2}-1\right)^{\delta} \sum_{w \cdot \lambda \in W \mathfrak{s}, k \in \mathbf{Z}} N(w \cdot \lambda, k) v^{k} c_{w \cdot \lambda} .
\end{aligned}
$$


From the definitions we have (using (b)):

$$
\begin{aligned}
\gamma\left({ }^{\prime} L\right) & =\sum_{j}(-1)^{j} \gamma\left({ }^{\prime} L^{j}\right) \\
& =\sum_{j}(-1)^{j} \sum_{w \cdot \lambda \in W \mathfrak{s}} \operatorname{dim} \bar{V}_{w \cdot \lambda, j}(-v)^{j} \gamma\left(L_{\lambda}^{\dot{w} \sharp}\langle|w|+\nu+2 \rho\rangle\right) \\
& =\sum_{j} \sum_{w \cdot \lambda \in W \mathfrak{s}} \operatorname{dim} \bar{V}_{w \cdot \lambda, j} v^{|w|} c_{w \cdot \lambda} v^{j-|w|-\nu-\rho} .
\end{aligned}
$$

From 2.15(b) we have $\gamma(L)=\left(v^{2}-1\right)^{\delta} \gamma\left({ }^{\prime} L\right)$ hence

$$
\begin{aligned}
\gamma(L) & =\left(v^{2}-1\right)^{\delta} \sum_{j} \sum_{(w, \lambda) \in W \mathfrak{s}} \operatorname{dim} \bar{V}_{w \cdot \lambda, j} c_{w \cdot \lambda} v^{j-\nu-2 \rho} \\
& =\left(v^{2}-1\right)^{\delta} \sum_{w \cdot \lambda \in W \mathfrak{s}, k \in \mathbf{Z}} N(w \cdot \lambda, k) v^{k} c_{w \cdot \lambda} .
\end{aligned}
$$

Since $c_{w \cdot \lambda}$ are linearly independent in $\mathbf{H}$, it follows that for any $w \cdot \lambda$ we have

$$
\sum_{j} \operatorname{dim} \bar{V}_{w \cdot \lambda, j} v^{j-\nu-2 \rho}=\sum_{k \in \mathbf{Z}} N(w \cdot \lambda, k) v^{k}=\sum_{k \in \mathbf{Z}} N(w \cdot \lambda,-k) v^{k}
$$

hence for any $j$ we have $\operatorname{dim} \bar{V}_{w \cdot \lambda, j}=N(w \cdot \lambda,-j+\nu+2 \rho)$, as required.

2.17. We preserve the setup of 2.15; let $J \subset[1, r]$. We set $L^{J}=L_{\boldsymbol{\lambda}}^{\boldsymbol{\omega}, J}$, ${ }^{\prime} L^{J}={ }^{\prime} L_{\boldsymbol{\lambda}}^{\omega, J}$. As in 2.16, we set $\delta=(r-1) \rho$.

We now analyze the complex $\mathfrak{L}^{\otimes(r-1)} \in \mathcal{D}_{m}$ (point). We can find free abelian groups $\mathcal{X}_{2 \delta-i}$ of rank $\left(\begin{array}{l}\delta \\ i\end{array}\right),(i \in \mathbf{Z})$ such that $\mathcal{X}_{2 \delta}=\mathbf{Z}$, complexes $R_{\leq 2 \delta-i} \in \mathcal{D}_{m}$ (point) $(i \in[0, \delta+1])$ and distinguished triangles

$$
\left(R_{\leq 2 \delta-i-1}, R_{\leq 2 \delta-i}, \mathcal{X}_{2 \delta-i} \otimes \overline{\mathbf{Q}}_{l}(i-\delta)[i-2 \delta]\right), \quad(i \in[0, \delta])
$$

in $\mathcal{D}_{m}$ (point) such that $R_{\leq 2 \delta}=\mathfrak{L}^{\otimes(r-1)}, R_{\leq \delta-1}=0$. It follows that for $i \in[0, \delta]$ we have distinguished triangles in $\mathcal{D}_{m}\left(\tilde{\mathcal{B}}^{2}\right)$ :

$$
\left(R_{\leq 2 \delta-i-1} \otimes^{\prime} L^{J}, R_{\leq 2 \delta-i} \otimes^{\prime} L^{J}, \mathcal{X}_{2 \delta-i}(i-\delta) \otimes^{\prime} L^{J}[i-2 \delta]\right)
$$

hence we have exact sequences

$$
\begin{aligned}
\ldots & \rightarrow \mathcal{X}_{2 \delta-i}(i-\delta) \otimes\left({ }^{\prime} L^{J}\right)^{-2 \delta+i+j-1} \rightarrow\left(R_{\leq 2 \delta-i-1} \otimes{ }^{\prime} L^{J}\right)^{j} \\
& \rightarrow\left(R_{\leq 2 \delta-i} \otimes{ }^{\prime} L^{J}\right)^{j} \rightarrow \mathcal{X}_{2 \delta-i}(i-\delta) \otimes\left({ }^{\prime} L^{J}\right)^{-2 \delta+i+j} \rightarrow \ldots
\end{aligned}
$$


Thus, setting

$$
\begin{aligned}
& \mathcal{R}_{i, j}=\left(R_{\leq 2 \delta-i} \otimes{ }^{\prime} L^{J}\right)^{j} \text { for } i \in[0, \delta+1], \\
& \mathcal{P}_{i, j}=\mathcal{X}_{2 \delta-i}(i-\delta) \otimes\left({ }^{\prime} L^{J}\right)^{-2 \delta+i+j} \text { for } i \in[0, \delta],
\end{aligned}
$$

we have $\mathcal{R}_{\delta+1, j}=0$ for all $j$ and, for any $i \in[0, \delta]$, we have an exact sequence in $\mathcal{M}_{m}\left(\tilde{\mathcal{B}}^{2}\right)$ :

(a) $\quad \cdots \rightarrow \mathcal{P}_{i, j-1} \rightarrow \mathcal{R}_{i+1, j} \rightarrow \mathcal{R}_{i, j} \rightarrow \mathcal{P}_{i, j} \rightarrow \mathcal{R}_{i+1, j+1} \rightarrow \mathcal{R}_{i, j+1} \rightarrow \ldots$

Note that for any $j$ we have

$$
\mathcal{R}_{0, j}=\left(L^{J}\right)^{j}
$$

$$
\mathcal{P}_{0, j}=\left({ }^{\prime} L^{J}\right)^{j-2 \delta}(-\delta)
$$

Indeed, (c) is obvious; (b) follows from 2.15(b):

$$
\mathcal{R}_{0, j}=\left(R_{\leq 2 \delta} \otimes^{\prime} L^{J}\right)^{j}=\left(\mathfrak{L}^{\otimes(r-1)} \otimes^{\prime} L^{J}\right)^{j}=\left(L^{J}\right)^{j} .
$$

2.18. We preserve the setup of 2.15; there is no assumption on $J$. The restriction of $M:=M_{\lambda}^{\boldsymbol{\omega},[1, r]}$ to $\tilde{\mathcal{O}}_{\mathbf{w}}^{J}$ (an open dense subset of $\tilde{\mathcal{O}}_{\mathbf{w}}^{[1, r]}$ ) is the same as the restriction of $M^{J}:=M_{\lambda}^{\boldsymbol{\omega}, J}$ to $\tilde{\mathcal{O}}_{\mathbf{w}}^{J}$; the restriction of $M$ to $\tilde{\mathcal{O}}_{\mathbf{w}}^{[1, r]}-\tilde{\mathcal{O}}_{\mathbf{w}}^{J}$ (a closed subset of $\left.\tilde{\mathcal{O}}_{\mathbf{w}}^{[1, r]}\right)$, extended by 0 on $\tilde{\mathcal{B}}^{r+1}-\left(\tilde{\mathcal{O}}_{\mathbf{w}}^{[1, r]}-\tilde{\mathcal{O}}_{\mathbf{w}}^{J}\right)$, is denoted by $\dot{M}^{J}$. We have a distinguished triangle

$$
\left(M^{J}, M, \dot{M}^{J}\right)
$$

in $\mathcal{D}_{m}\left(\tilde{\mathcal{B}}^{r+1}\right)$. We have the following result.

(b) Let $h \in \mathbf{Z}$. Let $K$ be either $\dot{M}^{J}$ or $M^{J}$. Any composition factor of $K^{h} \in \mathcal{M}\left(\tilde{\mathcal{B}}^{r+1}\right)$ is of the form $M_{\boldsymbol{\lambda}^{\prime}}^{\omega^{\prime},[1, r]}\left\langle\left|\mathbf{w}^{\prime}\right|+\nu+(r+1) \rho\right\rangle$ for some $\mathbf{w}^{\prime}=\left(w_{1}^{\prime}, w_{2}^{\prime}, \ldots, w_{r}^{\prime}\right) \in W^{r}, \lambda^{\prime}=\left(\lambda_{1}^{\prime}, \lambda_{2}^{\prime}, \ldots, \lambda_{r}^{\prime}\right) \in \mathfrak{s}^{r}$ such that $w_{i}=$ $w_{i}^{\prime}, \lambda_{i}=\lambda_{i}^{\prime}$ for all $i \in J$; here $\omega^{\prime}=\left(\dot{w}_{1}^{\prime}, \dot{w}_{2}^{\prime}, \ldots, \dot{w}_{r}^{\prime}\right)$.

It is enough to show that for any $h$, any composition factor of $\left(\theta^{*} K\right)^{h}(\theta$ as in 2.15) is of the form $\theta^{*} M_{\boldsymbol{\lambda}^{\prime}}^{\boldsymbol{\omega}^{\prime},[1, r]}\left\langle\left|\mathbf{w}^{\prime}\right|+\nu+(r+1) \rho\right\rangle$ for some $\mathbf{w}^{\prime}, \boldsymbol{\lambda}^{\prime}, \boldsymbol{\omega}^{\prime}$ as in (b). To see this we use the fact that $\left(\theta^{*} K\right)^{h}$ is a $\mathcal{G}$-equivariant perverse 
sheaf on $G^{r+1}$ (it is obtained from the $\mathcal{G}$-equivariant object $\tilde{M}_{\lambda}^{\omega}$ by operations which preserve $\mathcal{G}$-equivariance: passage to an intersection cohomology complex, restriction to a $\mathcal{G}$-invariant subvariety, taking a perverse cohomology sheaf) and that all simple $\mathcal{G}$-equivariant perverse sheaves on $G^{r+1}$ are of the form $\theta^{*} M_{\boldsymbol{\lambda}^{\prime}}^{\boldsymbol{\omega}^{\prime},[1, r]}\left\langle\left|\mathbf{w}^{\prime}\right|+\nu+(r+1) \rho\right\rangle$ with $\mathbf{w}^{\prime}, \boldsymbol{\lambda}^{\prime}, \boldsymbol{\omega}^{\prime}$ as in (b) (but with $\mathbf{w}^{\prime}$ unrestricted), see $2.15(\mathrm{~d})$. The fact that the $\mathbf{w}^{\prime}, \boldsymbol{\lambda}^{\prime}$ which appear are restricted as in (b) is immediate.

We show:

(c) $\left(\dot{M}^{J}\langle|\mathbf{w}|+\nu+(r+1) \rho-1\rangle\right)^{j}=0$ for any $j>0$.

It is enough to show that $\operatorname{dim} \operatorname{supp} \mathcal{H}^{h}\left(\dot{M}^{J}[|\mathbf{w}|+\nu+(r+1) \rho-1]\right) \leq-h$ for any $h \in \mathbf{Z}$. Assume first that $h \leq-|\mathbf{w}|-\nu-(r+1) \rho$. Since $M$ is an intersection cohomology complex with support of dimension $|\mathbf{w}|+\nu+(r+1) \rho$, we have $\operatorname{dim} \operatorname{supp} \mathcal{H}^{h-1}(M[|\mathbf{w}|+\nu+(r+1) \rho])<-h+1$ hence

$$
\operatorname{dimsupp} \mathcal{H}^{h-1}\left(\dot{M}^{J}[|\mathbf{w}|+\nu+(r+1) \rho]\right)<-h+1
$$

hence $\operatorname{dim} \operatorname{supp} \mathcal{H}^{h-1}\left(\dot{M}^{J}[|\mathbf{w}|+\nu+(r+1) \rho]\right) \leq-h$, hence

$$
\operatorname{dim} \operatorname{supp} \mathcal{H}^{h}\left(\dot{M}^{J}[|\mathbf{w}|+\nu+(r+1) \rho-1]\right) \leq-h .
$$

Next we assume that $h=-|\mathbf{w}|-\nu-(r+1) \rho+1$. Then

$$
\begin{gathered}
\operatorname{dimsupp} \mathcal{H}^{h-1}\left(\dot{M}^{J}[|\mathbf{w}|+\nu+(r+1) \rho]\right) \leq \operatorname{dim}\left(\tilde{\mathcal{O}}_{\mathbf{w}}^{[1, r]}-\tilde{\mathcal{O}}_{\mathbf{w}}^{J}\right) \leq \\
|\mathbf{w}|+\nu+(r+1) \rho-1=-h,
\end{gathered}
$$

hence

$$
\operatorname{dim} \operatorname{supp} \mathcal{H}^{h}\left(\dot{M}^{J}[|\mathbf{w}|+\nu+(r+1) \rho-1]\right) \leq-h .
$$

Finally, assume that $h \geq-|\mathbf{w}|-\nu-(r+1) \rho+2$. Then $\mathcal{H}^{h-1}(M[|\mathbf{w}|+\nu+$ $(r+1) \rho])=0$, hence $\mathcal{H}^{h-1}\left(\dot{M}^{J}[|\mathbf{w}|+\nu+(r+1) \rho]\right)=0$, hence $\mathcal{H}^{h}\left(\dot{M}^{J}[|\mathbf{w}|+\right.$ $\nu+(r+1) \rho-1])=0$. This proves $(\mathrm{c})$.

2.19. We preserve the setup of 2.15 ; there is no assumption on $J$. We shall need a variant of the results in 2.18. The restriction of ${ }^{\prime} M:={ }^{\prime} M_{\lambda}^{\boldsymbol{\omega},[1, r]}$ to ${ }^{\prime} \tilde{\mathcal{O}}_{\mathbf{w}}^{J}$ (an open dense subset of ${ }^{\prime} \tilde{\mathcal{O}}_{\mathbf{w}}^{[1, r]}$ ) is the same as the restriction of ${ }^{\prime} M^{J}:={ }^{\prime} M_{\boldsymbol{\lambda}}^{\boldsymbol{\omega}, J}$ to ${ }^{\prime} \tilde{\mathcal{O}}_{\mathbf{w}}^{J}$; the restriction of ${ }^{\prime} M$ to ${ }^{\prime} \tilde{\mathcal{O}}_{\mathbf{w}}^{[1, r]}-{ }^{\prime} \tilde{\mathcal{O}}_{\mathbf{w}}^{J}$ (a closed subset 
of $\left.{ }^{\prime} \tilde{\mathcal{O}}_{\mathbf{w}}^{[1, r]}\right)$, extended by 0 on ' $\tilde{\mathcal{B}}^{r+1}-\left({ }^{\prime} \tilde{\mathcal{O}}_{\mathbf{w}}^{[1, r]}-{ }^{\prime} \tilde{\mathcal{O}}_{\mathbf{w}}^{J}\right)$, is denoted by ${ }^{\prime} \dot{M}^{J}$. We have a distinguished triangle

$$
\left({ }^{\prime} M^{J},{ }^{\prime} M,{ }^{\prime} \dot{M}^{J}\right)
$$

in $\mathcal{D}\left({ }^{\prime} \tilde{\mathcal{B}}^{r+1}\right)$. The following result can be deduced from 2.18(b).

(b) Let $h \in \mathbf{Z}$. Let ' $K$ be either ${ }^{\prime} \dot{M}^{J}$ or ${ }^{\prime} M^{J}$. Any composition factor of $\left({ }^{\prime} K\right)^{h} \in \mathcal{M}\left({ }^{\prime} \tilde{\mathcal{B}}^{r+1}\right)$ is of the form ${ }^{\prime} M_{\boldsymbol{\lambda}^{\prime}}^{\boldsymbol{\omega}^{\prime},[1, r]}\left\langle\left|\mathbf{w}^{\prime}\right|+\nu+2 \rho\right\rangle$ for some $\mathbf{w}^{\prime}=$ $\left(w_{1}^{\prime}, w_{2}^{\prime}, \ldots, w_{r}^{\prime}\right) \in W^{r}, \lambda^{\prime}=\left(\lambda_{1}^{\prime}, \lambda_{2}^{\prime}, \ldots, \lambda_{r}^{\prime}\right) \in \mathfrak{s}^{r}$ such that $w_{i}=w_{i}^{\prime}$, $\lambda_{i}=\lambda_{i}^{\prime}$ for all $i \in J$, and $\boldsymbol{\lambda}^{\prime}$ is $\mathbf{w}$-adapted; here $\boldsymbol{\omega}^{\prime}=\left(\dot{w}_{1}^{\prime}, d w_{2}^{\prime}, \ldots, \dot{w}_{r}^{\prime}\right)$.

We note:

(c) $\left({ }^{\prime} \dot{M}^{J}\langle|\mathbf{w}|+\nu+2 \rho-1\rangle\right)^{j}=0$ for any $j>0$.

The proof is entirely similar to that of $2.18(\mathrm{c})$; alternatively it can be deduced from $2.18(\mathrm{c})$.

2.20. We preserve the setup of 2.15. Assume that $w_{u} \cdot \lambda_{u} \in \mathbf{c}$ for some $u \in J$. We set $L^{J}=L_{\boldsymbol{\lambda}}^{\boldsymbol{\omega}, J},{ }^{\prime} L^{J}={ }^{\prime} L_{\boldsymbol{\lambda}}^{\boldsymbol{\omega}, J}$. Let $\dot{M}^{J}$ be as in 2.18; let ${ }^{\prime} \dot{M}^{J}$ be as in 2.19. Let $\dot{L}^{J}=p_{0 r !} \dot{M}^{J}\langle|\mathbf{w}|\rangle \in \mathcal{D}\left(\tilde{\mathcal{B}}^{2}\right),{ }^{\prime} \dot{L}^{J}=\bar{p}_{0 r !}{ }^{\prime} \dot{M}^{J}\langle|\mathbf{w}|\rangle \in \mathcal{D}\left(\tilde{\mathcal{B}}^{2}\right)$. Let $j \in \mathbf{Z}$. We have the following results, in which $\mathcal{R}_{i, j}, \mathcal{P}_{i, j}$ are as in 2.17 with $J=[1, r]$ and $\delta=(r-1) \rho$.

(a) We have $\left(L^{J}\right)^{j} \in \mathcal{M} \preceq \tilde{\mathcal{B}}^{2}$. If $j>2 \delta+\nu+2 \rho+(r-1)$ a then $\left(L^{J}\right)^{j} \in \mathcal{M}^{\prec} \tilde{\mathcal{B}}^{2}$.

(b) We have $\left(\dot{L}^{J}\right)^{j} \in \mathcal{M} \preceq \tilde{\mathcal{B}}^{2}$. If $j \geq 2 \delta+\nu+2 \rho+(r-1)$ a then $\left(\dot{L}^{J}\right)^{j} \in \mathcal{M}^{\prec} \tilde{\mathcal{B}}^{2}$.

(c) We have $\left({ }^{\prime} L^{J}\right)^{j} \in \mathcal{M} \preceq \tilde{\mathcal{B}}^{2}$. If $j>\nu+2 \rho+(r-1)$ a then $\left({ }^{\prime} L^{J}\right)^{j} \in \mathcal{M}^{\prec} \tilde{\mathcal{B}}^{2}$.

(d) We have $\left({ }^{\prime} \dot{L}^{J}\right)^{j} \in \mathcal{M} \preceq \tilde{\mathcal{B}}^{2}$. If $j \geq \nu+2 \rho+(r-1)$ a then $\left({ }^{\prime} \dot{L}^{J}\right)^{j} \in \mathcal{M}^{\prec} \tilde{\mathcal{B}}^{2}$.

(e) If $i \in[0, \delta+1], J=[1, r]$, then $\mathcal{R}_{i, j} \in \mathcal{M} \preceq \tilde{\mathcal{B}}^{2}$.

(f) If $i \in[0, \delta+1], j>2 \delta-i+\nu+2 \rho+(r-1) a, J=[1, r]$, then $\mathcal{R}_{i, j} \in \mathcal{M}^{\prec} \tilde{\mathcal{B}}^{2}$.

(g) If $i \in[0, \delta], J=[1, r]$, then $\mathcal{P}_{i, j} \in \mathcal{M} \tilde{\mathcal{B}}^{2}$. If $i \in[0, \delta], j>2 \delta-i+\nu+$ $2 \rho+(r-1) a, J=[1, r]$, then $\mathcal{P}_{i, j} \in \mathcal{M}^{\prec} \tilde{\mathcal{B}}^{2}$.

We prove (e) by descending induction on $i$. If $i=\delta+1$ then, since $\mathcal{R}_{\delta+1, j}=0$, there is nothing to prove. Now assume that $i \in[0, \delta]$. Assume that $\mathbf{L}_{\lambda}^{\dot{w}}$ is a composition factor of $\mathcal{R}_{i, j}$ (without the mixed structure). We must show that $w \cdot \lambda \preceq \mathbf{c}$. By the induction hypothesis we can assume that $\mathbf{L}_{\lambda}^{\dot{w}}$ is not 
a composition factor of $\mathcal{R}_{i+1, j}$; hence by 2.17 (a) it is a composition factor of $\mathcal{P}_{i, j}$. Hence $\mathbf{L}_{\lambda}^{\dot{w}}$ is a composition factor of $\left({ }^{\prime} L^{[1, r]}\right)^{-2 \delta+i+j}$. Hence $\bar{V}_{w \cdot \lambda, k}$ in 2.16 is $\neq 0$ for some $k$. Using 2.16(c) we see that $N(w \cdot \lambda, k) \neq 0$ for some $k$. Using the definition of $N(w \cdot \lambda, k)$ we see that $w \cdot \lambda \preceq \mathbf{c}$ (recall that $w_{u} \cdot \lambda_{u} \in \mathbf{c}$ for some $u$ ) and (e) is proved.

We prove (f) by descending induction on $i$. If $i=\delta+1$ then, since $\mathcal{R}_{\delta+1, j}=0$, there is nothing to prove. Now assume that $i \in[0, \delta]$. Assume that $\mathbf{L}_{\lambda}^{\dot{w}}$ is a composition factor of $\mathcal{R}_{i, j}$ (without the mixed structure). We must show that $w \cdot \lambda \prec \mathbf{c}$. By the induction hypothesis we can assume that $\mathbf{L}_{\lambda}^{\dot{w}}$ is not a composition factor of $\mathcal{R}_{i+1, j}$ (we have $j>2 \delta-i-1+\nu+$ $2 \rho+(r-1) a)$; hence by $2.17(\mathrm{a}), \mathbf{L}_{\lambda}^{\dot{w}}$ is a composition factor of $\mathcal{P}_{i, j}$. Hence $\mathbf{L}_{\lambda}^{\dot{w}}$ is a composition factor of $\left({ }^{\prime} L^{[1, r]}\right)^{-2 \delta+i+j}$. Hence $\bar{V}_{w \cdot \lambda,-2 \delta+i+j}$ in 2.16 is $\neq 0$. Using $2.16(\mathrm{c})$ we see that $N(w \cdot \lambda, 2 \delta-i-j+\nu+2 \rho) \neq 0$. We have $2 \delta-i-j+\nu+2 \rho<-(r-1) a$. Using 1.10 (a) we deduce that $w \cdot \lambda \prec \mathbf{c}$ and (f) is proved.

We prove (g). This follows from the exact sequence 2.17(a) (with $J=$ $[1, r])$ using (e), (f).

We prove (a) assuming that $J=[1, r]$. From (e), (f) we have $\mathcal{R}_{0, j} \in$ $\mathcal{M} \preceq \tilde{\mathcal{B}}^{2}$ and $\mathcal{R}_{0, j} \in \mathcal{M}^{\prec} \tilde{\mathcal{B}}^{2}$ if $j>2 \delta+\nu+2 \rho+(r-1) a$. Using 2.17(b) we deduce that (a) holds (when $J=[1, r]$ ).

We prove (c) assuming that $J=[1, r]$. From (g) we have $\mathcal{P}_{0, j} \in \mathcal{M} \preceq \tilde{\mathcal{B}}^{2}$ and $\mathcal{P}_{0, j} \in \mathcal{M}^{\prec} \tilde{\mathcal{B}}^{2}$ if $j>2 \delta+\nu+2 \rho+(r-1) a$. Using 2.17(c) we deduce that $\left({ }^{\prime} L^{J}\right)^{j-2 \delta}(-\delta)$ is in $\mathcal{M}^{\prec} \tilde{\mathcal{B}}^{2}$ and is in $\mathcal{M}^{\prec} \tilde{\mathcal{B}}^{2}$ if $j-2 \delta>\nu+2 \rho+(r-1) a$. We deduce that (c) holds (when $J=[1, r]$ ).

We prove (b). Assume that $j \in \mathbf{Z}$ and $w \cdot \lambda \in \mathfrak{s}$ is such that $\mathbf{L}_{\lambda}^{\dot{w}}$ is a composition factor of $\left(\dot{L}^{J}\right)^{j}$ (without mixed structure). Then there exists $h$ such that $\mathbf{L}_{\lambda}^{\dot{w}}$ is a composition factor of $\left(p_{0 r !}\left(\dot{M}^{J}[|\mathbf{w}|]\right)^{h}[-h]\right)^{j}$. We have $\left(\dot{M}^{J}[|\mathbf{w}|)^{h} \neq 0\right.$ hence $\left(\dot{M}^{J}[|\mathbf{w}|+\nu+(r+1) \rho-1]\right)^{h-\nu-(r+1) \rho+1} \neq 0$, hence by $2.18(\mathrm{c}), h-\nu-(r+1) \rho+1 \leq 0$. From 2.18(b) we see that there exist $\mathbf{w}^{\prime}=\left(w_{1}^{\prime}, w_{2}^{\prime}, \ldots, w_{r}^{\prime}\right) \in W^{r}, \lambda^{\prime}=\left(\lambda_{1}^{\prime}, \lambda_{2}^{\prime}, \ldots, \lambda_{r}^{\prime}\right) \in \mathfrak{s}^{r}$ such that $w_{i}=w_{i}^{\prime}, \lambda_{i}=\lambda_{i}^{\prime}$ for all $i \in J$ and $\mathbf{L}_{\lambda}^{\dot{w}}$ is a composition factor of

$$
\left(p_{0 r !}\left(M_{\boldsymbol{\lambda}}^{\boldsymbol{\omega}^{\prime},[1, r]}\left[\left|\mathbf{w}^{\prime}\right|+\nu+(r+1) \rho\right][-h]\right)^{j}=L_{\boldsymbol{\lambda}^{\prime}}^{\boldsymbol{\omega}^{\prime},[1, r]}\left[\left|\mathbf{w}^{\prime}\right|\right]\right)^{j+\nu+(r+1) \rho-h}
$$

here $\boldsymbol{\omega}^{\prime}=\left(\dot{w}_{1}^{\prime}, \ldots, \dot{w}_{r}^{\prime}\right)$. From the part of (a) that is already proved (for $\mathbf{w}^{\prime}, \boldsymbol{\lambda}^{\prime}$ instead of $\left.\mathbf{w}, \boldsymbol{\lambda}\right)$ we see that $w \cdot \lambda \preceq \mathbf{c}$ and that if $j+\nu+(r+1) \rho-h>$ 
$2 \delta+\nu+2 \rho+(r-1) a$ that is, if $j>2 \delta+(r-1) \rho+(r-1) a+h$, then $w \cdot \lambda \prec \mathbf{c}$. If $j \geq 2 \delta+\nu+2 \rho+(r-1) a$ then, using that $0>h-\nu-(r+1) \rho$, we see that we have indeed $j>2 \delta+(r-1) \rho+(r-1) a+h$. This proves (b).

The proof of (d) is entirely similar to that of (b); it uses the already proved part of (c) and it uses the results of 2.19 instead of those in 2.18.

We prove (a) without assumption on $J$. Applying $p_{0 r}$ ! to 2.18(a) we get a distinguished triangle $\left(L^{J}, L^{[1, r]}, \dot{L}^{J}\right)$. This gives rise to an exact sequence

$$
\left(\dot{L}^{J}\right)^{j-1} \rightarrow\left(L^{J}\right)^{j} \rightarrow\left(L^{[1, r]}\right)^{j} \rightarrow\left(\dot{L}^{J}\right)^{j} .
$$

Using this together with (b) and the already proved part of (a) we see that (a) holds in general.

We prove (c) without assumption on $J$. Applying $\bar{p}_{0 r}$ ! to 2.19 (a) we get a distinguished triangle $\left({ }^{\prime} L^{J},{ }^{\prime} L^{[1, r]},{ }^{\prime} \dot{L}^{J}\right)$. This gives rise to an exact sequence

$$
\left({ }^{\prime} \dot{L}^{J}\right)^{j-1} \rightarrow\left({ }^{\prime} L^{J}\right)^{j} \rightarrow\left({ }^{\prime} L^{[1, r]}\right)^{j} \rightarrow\left({ }^{\prime} \dot{L}^{J}\right)^{j}
$$

Using this together with (d) and the already proved part of (c) we see that (c) holds in general.

2.21. Let $j \in \mathbf{Z}$. In (a), (b) below, $\mathcal{R}_{i, j}$ is as in 2.17 with arbitrary $J$.

(a) If $i \in[0, \delta+1]$ then $\mathcal{R}_{i, j} \in \mathcal{M} \preceq \tilde{\mathcal{B}}^{2}$.

(b) If $i \in[0, \delta+1], j>2 \delta-i+\nu+2 \rho+(r-1)$ a then $\mathcal{R}_{i, j} \in \mathcal{M}^{\prec} \tilde{\mathcal{B}}^{2}$.

Note that (a), (b) are generalizations of 2.20(e), 2.20(f) (which correspond to the case $J=[1, r])$.

We prove (a), (b) by descending induction on $i$. If $i=\delta+1$ then, since $\mathcal{R}_{\delta+1, j}=0$, there is nothing to prove. Now assume that $i \in[0, \delta]$. Assume that $\mathbf{L}_{\lambda}^{\dot{w}}$ is a composition factor of $\mathcal{R}_{i, j}$ (without the mixed structure). We must show that $w \cdot \lambda \preceq \mathbf{c}$ and that, if $j>2 \delta-i+\nu+2 \rho+(r-1) a$, then $w \cdot \lambda \prec \mathbf{c}$. Using 2.17(a), we see that $\mathbf{L}_{\lambda}^{\dot{w}}$ is a composition factor of $\mathcal{R}_{i+1, j}$ or of $\mathcal{P}_{i, j}$. In the first case, using the induction hypothesis we see that $w \cdot \lambda \preceq \mathbf{c}$ and that, if $j>2 \delta-i+\nu+2 \rho+(r-1) a$ (so that $j>2 \delta-i-1+\nu+2 \rho+(r-1) a$ ), then $w \cdot \lambda \prec \mathbf{c}$. In the second case, $\mathbf{L}_{\lambda}^{\dot{w}}$ is a composition factor of $\left({ }^{\prime} L^{J}\right)^{-2 \delta+i+j}$. Using $2.20(\mathrm{c})$ we see that $w \cdot \lambda \preceq \mathbf{c}$ and that, if $j>2 \delta-i+\nu+2 \rho+(r-1) a$ 
(so that $-2 \delta+i+j>\nu+2 \rho+(r-1) a$ ), then $w \cdot \lambda \prec \mathbf{c}$. This proves (a), (b).

2.22. We preserve the setup of 2.20 . In (a), (b) below we take $j=2 \delta+\nu+$ $2 \rho+(r-1) a$. We have the following results.

(a) We have canonically $\left.\underline{g r_{j}}\left(\left(L^{J}\right)^{j}\right) \stackrel{\sim}{\rightarrow} g r_{j}\left({ }^{\prime} L^{J}\right)^{j-2 \delta}(-\delta)\right)$.

(b) We have canonically $\underline{g r_{j}\left(\left(L^{J}\right)^{j}\right)} \stackrel{\sim}{\rightarrow} g r_{j}\left(\left(L^{[1, r]}\right)^{j}\right)$.

(c) If $\left(w_{1}, \lambda_{1}\right) \in \mathbf{c}, \ldots,\left(w_{r}, \lambda_{r}\right) \in \mathbf{c},(w, \lambda) \in \mathbf{c}$ and $j=2 \delta+\nu+\rho+(r-1) a$ then the multiplicity of $\mathbf{L}_{\lambda}^{\dot{w}}$ in $g r_{j}\left(\left(L^{J}\right)^{j}\right)$ is

$$
\sum h_{z_{1} \cdot \lambda_{1}^{\prime}, w_{2} \cdot \lambda_{2}, z_{2} \cdot \lambda_{2}^{\prime}}^{*} h_{z_{2} \cdot \lambda_{2}^{\prime}, w_{3} \cdot \lambda_{3}, z_{3} \cdot \lambda_{3}^{\prime}}^{*} \ldots h_{z_{r-1} \cdot \lambda_{r-1}^{\prime}, w_{r} \cdot \lambda_{r}, z_{r} \cdot \lambda_{r}^{\prime}}^{*}
$$

where the sum is taken over all $z_{1} \cdot \lambda_{1}^{\prime}, z_{2} \cdot \lambda_{2}^{\prime}, \ldots, z_{r} \cdot \lambda_{r}^{\prime}$ in $\mathbf{c}$ such that $z_{1} \cdot \lambda_{1}^{\prime}=w_{1} \cdot \lambda_{1}, z_{r} \cdot \lambda_{r}^{\prime}=w \cdot \lambda$.

(d) Assume that $i \in[0, \delta+1]$. Then $\mathcal{R}_{i, j}$ (notation of 2.17) is mixed of weight $\leq j-i$.

We prove (d) by descending induction on $i$. If $i=\delta+1$ there is nothing to prove. Assume now that $i \leq \delta$. By Deligne's theorem, ' $L^{J}$ is mixed of weight $\leq 0$; hence $\left({ }^{\prime} L^{J}\right)^{-2 \delta+i+j}$ is mixed of weight $\leq-2 \delta+i+j$ and $\mathcal{X}_{2 \delta-i}(i-\delta) \otimes\left({ }^{\prime} L^{J}\right)^{-2 \delta+i+j}$ is mixed of weight $\leq-2 \delta+i+j-2(i-\delta)=j-i$. In other words, $\mathcal{P}_{i, j}$ (notation of 2.17) is mixed of weight $\leq j-i$. Thus in the exact sequence $\mathcal{R}_{i+1, j} \rightarrow \mathcal{R}_{i, j} \rightarrow \mathcal{P}_{i, j}$ coming from 2.17 (a) in which $\mathcal{R}_{i+1, j}$ is mixed of weight $\leq j-i-1<j-i$ (by the induction hypothesis) and $\mathcal{P}_{i, j}$ is mixed of weight $\leq j-i$ we must have that $\mathcal{R}_{i, j}$ is mixed of weight $\leq j-i$. This proves $(\mathrm{d})$.

We prove (a). From 2.17(a) we deduce an exact sequence

$$
g r_{j}\left(\mathcal{R}_{1, j}\right) \rightarrow g r_{j}\left(\mathcal{R}_{0, j}\right) \rightarrow g r_{j}\left(\mathcal{P}_{0, j}\right) \rightarrow g r_{j}\left(\mathcal{R}_{1, j+1}\right)
$$

By $\left(\right.$ d) we have $g r_{j}\left(\mathcal{R}_{1, j}\right)=0$. We have $g r_{j}\left(\mathcal{R}_{0, j}\right)=g r_{j}\left(\left(L^{J}\right)^{j}\right), g r_{j}\left(\mathcal{P}_{0, j}\right)=$ $g r_{j}\left(\left({ }^{\prime} L^{J}\right)^{-2 \delta+j}(-\delta)\right)$. Moreover, by $2.21(\mathrm{~b})$ we have $\mathcal{R}_{1, j+1} \in \mathcal{D}^{\prec} \tilde{\mathcal{B}}^{2}$ since $j+1>2 \delta-1+\nu+2 \rho+(r-1) a$. It follows that $\operatorname{gr}_{j}\left(\mathcal{R}_{1, j+1}\right) \in \mathcal{D}^{\prec} \tilde{\mathcal{B}}^{2}$. Thus the exact sequence above induces an isomorphism as in (a). 
We prove (b). As in 2.20 we have an exact sequence

$$
\left(\dot{L}^{J}\right)^{j-1} \rightarrow\left(L^{J}\right)^{j} \rightarrow\left(L^{[1, r]}\right)^{j} \rightarrow\left(\dot{L}^{J}\right)^{j} .
$$

This gives rise to an exact sequence

$$
g r_{j}\left(\left(\dot{L}^{J}\right)^{j-1}\right) \rightarrow g r_{j}\left(\left(L^{J}\right)^{j}\right) \rightarrow g r_{j}\left(\left(L^{[1, r]}\right)^{j}\right) \rightarrow g r_{j}\left(\left(\dot{L}^{J}\right)^{j}\right) .
$$

From 2.20(b) we have $g r_{j}\left(\left(\dot{L}^{J}\right)^{j}\right) \in \mathcal{M}^{\prec}\left(\tilde{\mathcal{B}}^{2}\right)$. It is then enough to show that $g r_{j}\left(\left(\dot{L}^{J}\right)^{j-1}\right)=0$. Since $\dot{M}^{J}\langle|\mathbf{w}|\rangle$ is the restriction of a pure complex of weight 0 to a subspace, it is mixed of weight $\leq 0$ (see [1, 5.1.14]). Hence $\dot{L}^{J}=\left(p_{0 r !} \dot{M}^{J}\langle|\mathbf{w}|\rangle\right.$ is mixed of weight $\leq 0$ and $\left(\dot{L}^{J}\right)^{j-1}$ is mixed of weight lej-1 (see [1, 5.4.1]). Thus, $g r_{j}\left(\left(\dot{L}^{J}\right)^{j-1}\right)=0$ as required. This proves (b).

We prove (c). By (a) and (b), the multiplicity in (c) is equal to the multiplicity of $\mathbf{L}_{\lambda}^{\dot{w}}$ in

$$
\left.g r_{j}\left({ }^{\prime} L^{[1, r]}\right)^{j-2 \delta}(-\delta)\right)=\left({ }^{\prime} L^{[1, r]}\right)^{j-2 \delta}(-\delta)
$$

(we use the fact that ${ }^{\prime} L^{[1, r]}$ is pure of weight zero); thus it is equal to $\bar{V}_{w \cdot \lambda, j-2 \delta}=\bar{V}_{w \cdot \lambda, \nu+2 \rho+(r-1) a}$ hence also to $N(w \cdot \lambda,-(r-1) a)$ (see 2.16(c)). It remains to use the equality $1.10(\mathrm{c})$.

2.23. Let $L, L^{\prime} \in \mathcal{D}^{\wedge} \tilde{\mathcal{B}}^{2}$. We show:

(a) Assume that $L, L^{\prime} \in \mathcal{M}^{\wedge} \tilde{\mathcal{B}}^{2}$ and that either $L$ or $L^{\prime}$ is in $\mathcal{D} \preceq \tilde{\mathcal{B}}^{2}$. If $j>a-\nu$ then $\left(L \circ L^{\prime}\right)^{j} \in \mathcal{M}^{\prec} \tilde{\mathcal{B}}^{2}$.

We can assume that $L=\mathbf{L}_{\lambda}^{\dot{w}}, L^{\prime}=\mathbf{L}_{\lambda^{\prime}}^{\dot{w}^{\prime}}$ and that either $w \cdot \lambda \in \mathbf{c}$ or $w^{\prime} \cdot \lambda^{\prime} \in \mathbf{c}$. According to 2.20 (b) we have

$$
\left(L_{\lambda}^{\dot{w} \sharp}\langle|w|\rangle \circ L_{\lambda^{\prime}}^{\dot{w}^{\prime} \sharp}\left\langle\left|w^{\prime}\right|\right\rangle\right)^{j^{\prime}} \in \mathcal{M}^{\prec} \tilde{\mathcal{B}}^{2}
$$

if $j^{\prime}>4 \rho+\nu+a$. Hence

$$
\left(L_{\lambda}^{\dot{w} \sharp}\langle|w|+\nu+2 \rho\rangle \circ L_{\lambda^{\prime}}^{\dot{w}^{\prime} \sharp}\left\langle\left|w^{\prime}\right|+\nu+2 \rho\right\rangle\right)^{j} \in \mathcal{M}^{\prec} \tilde{\mathcal{B}}^{2}
$$

if $j+2 \nu+4 \rho>4 \rho+\nu+a$ that is, if $j>a-\nu$. This proves (a).

(b) If $L \in \mathcal{D}^{\preceq} \tilde{\mathcal{B}}^{2}$ or $L^{\prime} \in \mathcal{D} \preceq \tilde{\mathcal{B}}^{2}$ then $L \circ L^{\prime} \in \mathcal{D} \preceq \tilde{\mathcal{B}}^{2}$. If $L \in \mathcal{D}^{\prec} \tilde{\mathcal{B}}^{2}$ or $L^{\prime} \in \mathcal{D}^{\prec} \tilde{\mathcal{B}}^{2}$ then $L \circ L^{\prime} \in \mathcal{D}^{\prec} \tilde{\mathcal{B}}^{2}$. 
The first assertion of (b) is shown in the same way as (a). The second assertion of (b) can be reduced to the first assertion.

2.24. For $L, L^{\prime} \in \mathcal{C}_{0}^{\mathbf{c}} \tilde{\mathcal{B}}^{2}$ we set

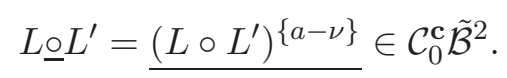

(For the notation ${ }^{\{i\}}$ see 0.2.) Now let $L, L^{\prime}, L^{\prime \prime} \in \mathcal{C}_{0}^{\mathbf{c}} \tilde{\mathcal{B}}^{2}$. By $2.23(\mathrm{~b})$, we have $L^{\prime} \circ L^{\prime \prime} \in \mathcal{D}_{\bar{m}}^{\preceq} \tilde{\mathcal{B}}^{2}$ and the functor $\Phi: \mathcal{D}_{\bar{m}}^{\preceq} \tilde{\mathcal{B}}^{2} \rightarrow \mathcal{D}_{\bar{m}}^{\preceq} \tilde{\mathcal{B}}^{2}, \Phi(K)=L \circ K$, is well defined. We note that, by $2.23(\mathrm{a})$, (i), (ii) below hold.

(i) If $X_{0} \in \mathcal{M} \frac{\prec}{m} \tilde{\mathcal{B}}^{2}$ then $\left(\Phi\left(X_{0}\right)\right)^{h} \in \mathcal{M}_{m}^{\prec} \tilde{\mathcal{B}}^{2}$ for any $h>a-\nu$.

(ii) $L^{\prime} \circ L^{\prime \prime}$ is mixed of weight $\leq 0$ and $\left(L^{\prime} \circ L^{\prime \prime}\right)^{h} \in \mathcal{M}_{m}^{\prec} \tilde{\mathcal{B}}^{2}$ for any $h>a-\nu$.

Similarly we have $L \circ L^{\prime} \in \mathcal{D} \frac{\preceq}{\bar{m}} \tilde{\mathcal{B}}^{2}$ and the functor $\Phi^{\prime}: \mathcal{D}_{\bar{m}}^{\preceq} \tilde{\mathcal{B}}^{2} \rightarrow \mathcal{D}_{\bar{m}}^{\preceq} \tilde{\mathcal{B}}^{2}$, $\Phi^{\prime}(K)=K \circ L^{\prime}$, is well defined. Moreover, (iii), (iv) below hold.

(iii) If $X_{0} \in \mathcal{M} \frac{\prec}{m} \tilde{\mathcal{B}}^{2}$ then $\left(\Phi^{\prime}\left(X_{0}\right)\right)^{h} \in \mathcal{M}_{m}^{\prec} \tilde{\mathcal{B}}^{2}$ for any $h>a-\nu$.

(iv) $L \circ L^{\prime}$ is mixed of weight $\leq 0$ and $\left(L \circ L^{\prime}\right)^{h} \in \mathcal{M}_{m}^{\prec} \tilde{\mathcal{B}}^{2}$ for any $h>a-\nu$.

We now apply [24, 1.12] with $Y_{1}, Y_{2}$ replaced by $\tilde{\mathcal{B}}^{2}$ we see that

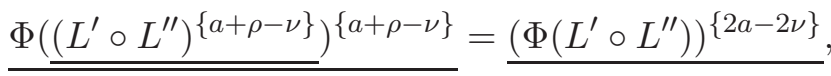

$$
\begin{aligned}
& \Phi^{\prime}\left(\underline{\left(L \circ L^{\prime}\right)^{\{a+\rho-\nu\}}}\right)^{\{a+\rho-\nu\}}=\underline{\left(\Phi^{\prime}\left(L \circ L^{\prime}\right)\right)^{\{2 a-2 \nu\}}} .
\end{aligned}
$$

Thus, we have

$$
\begin{aligned}
& L \underline{\circ}\left(L^{\prime} \underline{\circ} L^{\prime \prime}\right)=\underline{\left(L \circ L^{\prime} \circ L^{\prime \prime}\right)^{\{2 a-2 \nu\}},} \\
& \left(L \underline{\circ} L^{\prime}\right) \underline{\circ} L^{\prime \prime}=\underline{\left(L \circ L^{\prime} \circ L^{\prime \prime}\right)^{\{2 a-2 \nu\}}} .
\end{aligned}
$$

Hence

$$
L \underline{\circ}\left(L^{\prime} \underline{\varrho} L^{\prime \prime}\right)=\left(L \underline{\circ} L^{\prime}\right) \underline{\circ} L^{\prime \prime} .
$$

We see that $L, L^{\prime} \mapsto L \propto \underline{\circ} L^{\prime}$ defines a monoidal structure on $\mathcal{C}_{0}^{\mathbf{c}} \tilde{\mathcal{B}}^{2}$. Hence if ${ }^{1} L,{ }^{2}, \ldots,{ }^{r} L$ are in $\mathcal{C}_{0}^{\mathbf{c}} \tilde{\mathcal{B}}^{2}$ then ${ }^{1} L \underline{\circ}^{2} L \underline{\circ} \ldots \underline{\circ}^{r} L \in \mathcal{C}_{0}^{\mathbf{c}} \tilde{\mathcal{B}}^{2}$ is well defined; using [24, 1.12] repeatedly, we have

$$
{ }^{1} L \underline{\circ}^{2} L \underline{\circ} \ldots \underline{\circ}^{r} L=\left({ }^{1} L \circ{ }^{2} L \circ \ldots \circ{ }^{r} L\right)^{\{(r-1)(a-\nu)\}} .
$$


2.25. Let $L_{0}, L_{1} \in \mathcal{C}_{0}^{\mathbf{c}} \tilde{\mathcal{B}}^{2}$. We show that we have canonically

$$
\mathfrak{D}\left(L_{0} \text { 을 } L_{1}\right)=\mathfrak{D}\left(L_{0}\right) \underline{\circ} \mathfrak{D}\left(L_{1}\right) .
$$

(Note that in the right hand side, $\underline{\circ}$ is relative to $\tilde{\mathbf{c}}$ instead of $\mathbf{c}$, see below.) We can assume that $L_{i}=\mathbf{L}_{\lambda_{i}}^{\dot{w}_{i}}(i=0,1)$ where $w_{i} \cdot \lambda_{i} \in \mathbf{c}(i=0,1)$. Let $\tilde{L}_{i}=\mathfrak{D}\left(L_{i}\right)=\mathbf{L}_{\lambda_{i}^{-1}}^{\dot{w}_{i}}, i=0,1$. Note that $w_{i} \cdot \lambda_{i}^{-1} \in \tilde{\mathbf{c}}$ (see 1.14) and $\tilde{L}_{0}, \tilde{L}_{1} \in \mathcal{C}_{0}^{\tilde{\mathbf{c}}} \tilde{\mathcal{B}}^{2}$. It is enough to show that

$$
\mathfrak{D}\left(L_{0} \underline{\circ} L_{1}\right)=\tilde{L}_{0} \text { 은 } \tilde{L}_{1}
$$

If $w_{1}\left(\lambda_{1}\right) \neq \lambda_{0}$ (that is, $w_{1}\left(\lambda_{1}^{-1}\right) \neq \lambda_{0}^{-1}$ ), we have $L_{0} \circ L_{1}=0, \tilde{L}_{0} \circ \tilde{L}_{1}=0$ hence both sides of (a) are zero. Now assume that $w_{1}\left(\lambda_{1}\right)=\lambda_{0}$. Let $L=$ $L_{\boldsymbol{\lambda}}^{\boldsymbol{\omega}, J},{ }^{\prime} L={ }^{\prime} L_{\boldsymbol{\lambda}}^{\boldsymbol{\omega}, J},{ }^{\prime} M={ }^{\prime} M_{\boldsymbol{\lambda}}^{\boldsymbol{\omega}, J}$ where $\boldsymbol{\omega}=\left(\dot{w}_{0}, \dot{w}_{1}\right), \boldsymbol{\lambda}=\left(\lambda_{0}, \lambda_{1}\right), J=\{1\}$. Let $\tilde{L}=L_{\boldsymbol{\lambda}^{\prime}}^{\boldsymbol{\omega}, J},{ }^{\prime} \tilde{L}={ }^{\prime} L_{\boldsymbol{\lambda}^{\prime}}^{\boldsymbol{\omega}, J}{ }^{\prime} \tilde{M}={ }^{\prime} \tilde{M}_{\boldsymbol{\lambda}^{\prime}}^{\boldsymbol{\omega}, J}$ where $\boldsymbol{\lambda}^{\prime}=\left(\lambda_{0}^{-1}, \lambda_{1}^{-1}\right)$. By definition we have

$$
L=\left(L_{0}\left\langle-\left|w_{0}\right|-\nu-2 \rho\right\rangle \circ L_{1}\left\langle-\left|w_{1}\right|-\nu-2 \rho\right\rangle\right)\left\langle\left|w_{0}+\right| w_{1} \mid\right\rangle
$$

hence $L=\left(L_{0} \circ L_{1}\right)\langle-2 \nu-4 \rho\rangle$. Thus,

$$
L^{4 \rho+\nu+a}=\left(L_{0} \circ L_{1}\right)^{a-\nu}(-\nu-2 \rho)
$$

and

$$
g r_{4 \rho+\nu+a}\left(L^{4 \rho+n u+a}\right)=g r_{a-\nu}\left(L_{0} \circ L_{1}\right)^{a-\nu}(-\nu-2 \rho) .
$$

By 2.22(a) we have

$$
\underline{g r_{4 \rho+\nu+a}\left(L^{4 \rho+n u+a}\right)}=\underline{L^{\nu+2 \rho+a}}(-\rho)
$$

hence

$$
\underline{g r_{a-\nu}\left(L_{0} \circ L_{1}\right)^{a-\nu}}((a-\nu) / 2)={ }^{\prime} L^{\nu+2 \rho+a}((a+2 \rho+\nu) / 2) .
$$

Similarly,

$$
\underline{g r_{a-\nu}\left(\tilde{L}_{0} \circ \tilde{L}_{1}\right)^{a-\nu}}((a-\nu) / 2)=\underline{{ }^{\prime} \tilde{L}^{\nu+2 \rho+a}}((a+2 \rho+\nu) / 2) .
$$


It is then enough to show that

$$
\mathfrak{D}\left({ }^{\prime} \underline{L}^{\nu+2 \rho+a}((a+2 \rho+\nu) / 2)\right)=\underline{{ }^{\prime} \tilde{L}^{\nu+2 \rho+a}}((a+2 \rho+\nu) / 2) .
$$

This would follow from the stronger result that

$$
\mathfrak{D}\left({ }^{\prime} L^{\nu+2 \rho+a}\right)={ }^{\prime} \tilde{L}^{\nu+2 \rho+a}(a+2 \rho+\nu) .
$$

Recall that ${ }^{\prime} L=\bar{p}_{0 r !}\left({ }^{\prime} M\right)\left\langle\left|w_{0}\right|+\left|w_{1}\right|\right\rangle$ and similarly ${ }^{\prime} \tilde{L}=\bar{p}_{0 r !}\left({ }^{\prime} \tilde{M}\right)\left\langle\left|w_{0}\right|+\left|w_{1}\right|\right\rangle$ where ${ }^{\prime} M\left\langle\left|w_{0}\right|+\left|w_{1}\right|+\nu+2 \rho\right\rangle,{ }^{\prime} \tilde{M}\left\langle\left|w_{0}\right|+\left|w_{1}\right|+\nu+2 \rho\right\rangle$ are perverse sheaves, each being $\mathfrak{D}$ of the other. Since $\bar{p}_{01}$ is proper, $\bar{p}_{01}$ ! commutes with $\mathfrak{D}$. It follows that

$$
\mathfrak{D}\left({ }^{\prime} L\langle\nu+2 \rho\rangle\right)={ }^{\prime} \tilde{L}\langle\nu+2 \rho\rangle,
$$

hence

$$
\mathfrak{D}\left(\left({ }^{\prime} L\langle\nu+2 \rho\rangle\right)^{j}\right)=\left({ }^{\prime} \tilde{L}\langle\nu+2 \rho\rangle\right)^{-j},
$$

that is

$$
\mathfrak{D}\left({ }^{\prime} L^{\nu+2 \rho+j}\right)={ }^{\prime} \tilde{L}^{\nu+2 \rho-j}(\nu+2 \rho)
$$

for any $j$; in particular,

$$
\mathfrak{D}\left({ }^{\prime} L^{a+2 \nu+\rho}\right)={ }^{\prime} \tilde{L}^{-a+\nu+2 \rho}(\nu+2 \rho) .
$$

Thus it is enough to prove

$$
{ }^{\prime} \tilde{L}^{-a+\nu+2 \rho}(\nu+2 \rho)={ }^{\prime} \tilde{L}^{\nu+2 \rho+a}(a+2 \rho+\nu),
$$

that is

$$
{ }^{\prime} \tilde{L}^{-a+\nu+2 \rho}={ }^{\prime} \tilde{L}^{\nu+2 \rho+a}(a) .
$$

From the hard Lefschetz theorem applied to the projective morphism $\bar{p}_{01}$ and to ${ }^{\prime} \tilde{M}\left\langle\left|w_{0}\right|+\left|w_{1}\right|+\nu+2 \rho\right\rangle$ (a perverse sheaf of pure weight 0 ) we have canonically for any $i$ :

$$
{ }^{\prime} \tilde{L}^{\nu+2 \rho-i}={ }^{\prime} \tilde{L}^{\nu+2 \rho+i}(i) .
$$

Taking $i=a$ we obtain the desired result. This proves (a).

2.26. Let $r \geq 1$ and let $\mathbf{w}=\left(w_{1}, w_{2}, \ldots, w_{r}\right) \in W^{r}, \boldsymbol{\lambda}=\left(\lambda_{1}, \lambda_{2}, \ldots, \lambda_{r}\right) \in \mathfrak{s}^{r}$ and let $\boldsymbol{\omega}=\left(\omega_{1}, \omega_{2}, \ldots, \omega_{r}\right)$ be such that $\omega_{i} \in \kappa_{q}^{-1}\left(w_{i}\right)$ for $i=1, \ldots, r$. 
(a) Assume that $w_{i} \cdot \lambda_{i} \in \mathbf{c}$ for some $i \in[1, r]$ and that

$$
\underline{\left(\mathbf{L}_{\lambda_{1}}^{\omega_{1}} \circ \mathbf{L}_{\lambda_{2}}^{\omega_{2}} \circ \ldots \circ \mathbf{L}_{\lambda_{r}}^{\omega_{r}}\right)^{\{(r-1)(a-\nu)\}} \neq 0 .}
$$

Then $w_{i} \cdot \lambda_{i} \in \mathbf{c}$ for all $i \in[1, r]$.

Let $j=\nu+2 r \rho+(r-1) a$. By assumption we have $\underline{g r_{j}}\left(\left(L_{\boldsymbol{\lambda}}^{\boldsymbol{\omega},[1, r]}\right)^{j}\right) \neq 0$. Hence by $2.22(\mathrm{a})$ we have

$$
\underline{g r_{j}\left(\left(L_{\boldsymbol{\lambda}}^{\boldsymbol{\omega},[1, r]}\right)^{j-2(r-1) \rho}(-(r-1) \rho)\right)} \neq 0 .
$$

Thus there exists $w \cdot \lambda \in \mathbf{c}$ such that $\mathbf{L}_{\lambda}^{\dot{w}}$ has nonzero multiplicity in

$$
\left({ }^{\prime} L_{\boldsymbol{\lambda}}^{\boldsymbol{\omega},[1, r]}\right)^{j-2(r-1) \rho},
$$

that is, $\bar{V}_{w \cdot \lambda, j-2(r-1) \rho} \neq 0$ (notation of 2.16). Using 2.16(c) we see that $N(w \cdot \lambda,-j+2 \rho+\nu) \neq 0$ that is $N(w \cdot \lambda,-(r-1) a) \neq 0$. Using now 1.10(a) we see that $w_{i} \cdot \lambda_{i} \in \mathbf{c}$ for all $i \in[1, r]$.

\section{Sheaves on the Variety $Z$}

3.1. Let

$$
Z=\left\{\left(B, B^{\prime}, g U_{B}\right) ;\left(B, B^{\prime}\right) \in \mathcal{B}^{2}, g \in G, g B g^{-1}=B^{\prime}\right\}
$$

We define $\epsilon: \tilde{\mathcal{B}}^{2} \rightarrow Z$ by $(x \mathbf{U}, y \mathbf{U}) \mapsto\left(x \mathbf{B} x^{-1}, y \mathbf{B} y^{-1}, y \mathbf{U} x^{-1}\right)$. Now $\epsilon$ identifies $Z$ with $\mathbf{T} \backslash \tilde{\mathcal{B}}^{2}$ where $\mathbf{T}$ acts on $\tilde{\mathcal{B}}^{2}$ by $t:(x \mathbf{U}, y \mathbf{U}) \mapsto(x t \mathbf{U}, y t \mathbf{U})$. Note that $Z$ inherits an $\mathbf{F}_{q}$-structure from $\mathcal{B} \times \mathcal{B} \times G$.

3.2. The $G \times \mathbf{T}^{2}$-action on $\tilde{\mathcal{B}}^{2}$ (see 2.1) induces a $G \times \mathbf{T}^{2}$-action on $\mathbf{T} \backslash \tilde{\mathcal{B}}^{2}$ (see 3.1) hence a $G \times \mathbf{T}^{2}$-action on $Z$ in which the subgroup $\left\{\left(1, t_{1}, t_{2}\right) \in\right.$ $\left.G \times \mathbf{T}^{2} ; t_{1}=t_{2}\right\}$ acts trivially. For $w \in W$ let $Z_{w}=\left\{\left(B, B^{\prime}, g U_{B}\right) ;\left(B, B^{\prime}\right) \in\right.$ $\left.\mathcal{O}_{w}, g \in G, g B g^{-1}=B^{\prime}\right\}$; this is a single $G \times \mathbf{T}^{2}$-orbit on $Z$ with closure

$$
\bar{Z}_{w}=\left\{\left(B, B^{\prime}, g U_{B}\right) ;\left(B, B^{\prime}\right) \in \overline{\mathcal{O}}_{w}, g \in G, g B g^{-1}=B^{\prime}\right\}
$$

and we have $Z=\sqcup_{w \in W} Z_{w}$. Note that $Z_{w}=\epsilon\left(\tilde{\mathcal{O}}_{w}\right), \tilde{\mathcal{O}}_{w}=\epsilon^{-1}\left(Z_{w}\right), \bar{Z}_{w}=$ $\epsilon\left(\overline{\tilde{\mathcal{O}}}_{w}\right), \overline{\tilde{\mathcal{O}}}_{w}=\epsilon^{-1}\left(\bar{Z}_{w}\right)$. 
Let $\omega \in \kappa_{q}^{-1}(w)$. We have a diagram $\mathbf{T} \stackrel{j_{\omega}}{\rightarrow} \tilde{\mathcal{O}}_{w} \stackrel{\epsilon_{w}}{\leftarrow} Z_{w}$ where $\epsilon_{w}$ is the restriction of $\epsilon$ and $j_{\omega}$ is as in 2.1. Let $\lambda \in \mathfrak{s}$ be such that $w(\lambda)=\lambda$. The T-action on $\tilde{\mathcal{B}}^{2}$ in 3.1 is compatible under $j_{\omega}$ with the $\mathbf{T}$-action $t: t^{\prime} \mapsto$ $w^{-1}\left(t^{-1}\right) t^{\prime} t$ on $\mathbf{T}$ and $L_{\lambda}$ is equivariant for this action (by 1.4(a) with $w$ replaced by $w^{-1}$ ) hence $j_{\omega}^{*} L_{\lambda}$ is $\mathbf{T}$-equivariant so that there is a well defined local system $\mathcal{L}_{\lambda}^{\omega}$ of rank 1 on $Z_{w}$ such that $\epsilon_{w}^{*} \mathcal{L}_{\lambda}^{\omega}=j_{\omega}^{*} L_{\lambda}$. Note that the induced action of $\mathbf{T}_{n}^{2}$ (which acts trivially on $Z_{w}$ ) on any stalk of $\mathcal{L}_{\lambda}^{\omega}$ is via the character $\left(t_{1}, t_{2}\right) \mapsto \lambda^{-1}\left(t_{1}\right) \lambda\left(t_{2}\right)$. Moreover, $\mathcal{L}_{\lambda}^{\omega}$ is naturally pure of weight zero. We have $\epsilon_{w}^{*} \mathcal{L}_{\lambda}^{\omega}=L_{\lambda}^{\omega}$.

We show the converse:

(a) Let $\mathcal{L}$ be an irreducible $G \times \mathbf{T}^{2}$-equivariant local system on $Z_{w}$. Then $\mathcal{L}$ is isomorphic to $\mathcal{L}_{\lambda}^{\omega}$ for a unique $\lambda \in \mathfrak{s}$ such that $w(\lambda)=\lambda$.

The local system $\epsilon_{w}^{*} \mathcal{L}$ on $\tilde{\mathcal{B}}^{2}$ is irreducible and $G \times \mathbf{T}^{2}$-equivariant hence, by 2.1 , is isomorphic to $L_{\lambda}^{\omega}$ for a well defined $\lambda \in \mathfrak{s}$. Now the restriction of $\epsilon_{w}^{*} \mathcal{L}$ to any fibre of $\epsilon_{w}$ is the constant sheaf. On the other hand the restriction of $L_{\lambda}^{\omega}$ to any fibre of $\epsilon_{w}$ is (under an identification with $\mathbf{T}$ ) of the form $L_{w\left(\lambda^{-1}\right) \lambda}$ which is trivial if and only if $w(l)=\lambda$. We see that we must have $w(\lambda)=\lambda$. We have $\epsilon_{w}^{*} \mathcal{L} \cong \epsilon_{w}^{*} \mathcal{L}_{\lambda}^{\omega}$ (both are $\left.L_{\lambda}^{\omega}\right)$ hence $\mathcal{L} \cong \mathcal{L}_{\lambda}^{\omega}$. This proves (a).

We define $\mathfrak{h}: Z \rightarrow Z$ by $\left(B, B^{\prime}, g U_{B}\right) \mapsto\left(B^{\prime}, B, g^{-1} U_{B^{\prime}}\right)$. Note that $\mathfrak{h} \epsilon=\epsilon \tilde{\mathfrak{h}}: \tilde{\mathcal{B}}^{2} \rightarrow Z$. For $L \in \mathcal{D}_{m} Z$ we set $L^{\dagger}=\mathfrak{h}^{*} L$.

3.3. Let $\breve{W} \mathfrak{s}=\{w \cdot \lambda \in W \mathfrak{s} ; w(\lambda)=\lambda\}, \breve{c}=\breve{W} \mathfrak{s} \cap \mathbf{c}$. For $w \cdot \lambda \in \breve{W} \mathfrak{s}$ and $\omega \in \kappa_{q}^{-1}(w)$ we shall view $\mathcal{L}_{\lambda}^{\omega}$ as a constructible sheaf on $Z$ which is 0 on $Z-Z_{w}$. Let $\mathcal{L}_{\lambda}^{\omega \sharp}$ be its extension to an intersection cohomology complex of $\bar{Z}_{w}$, viewed as a complex on $Z$, equal to 0 on $Z-\bar{Z}_{w}$. Let

$$
\mathbb{L}_{\lambda}^{\omega}=\mathcal{L}_{\lambda}^{\omega \sharp}\langle|w|+\nu+\rho\rangle,
$$

a simple perverse sheaf on $Z$. Note that $\mathcal{L}_{\lambda}^{\omega}$ (resp. $\mathbb{L}_{\lambda}^{\omega}$ ) is noncanonically isomorphic to $\mathcal{L}_{\lambda}^{\dot{w}}\left(\operatorname{resp} . \mathbb{L}_{\lambda}^{\dot{w}}\right.$.)

We define $\tilde{\epsilon}: \mathcal{D}(Z) \rightarrow \mathcal{D}\left(\tilde{\mathcal{B}}^{2}\right)$ and $\tilde{\epsilon}: \mathcal{D}_{m}(Z) \rightarrow \mathcal{D}_{m}\left(\tilde{\mathcal{B}}^{2}\right)$ by

$$
\tilde{\epsilon}(L)=\epsilon^{*}(L)\langle\rho\rangle .
$$


From the definition we have

$$
\epsilon^{*} \mathcal{L}_{\lambda}^{\omega \sharp}=L_{\lambda}^{\omega \sharp}, \quad \tilde{\epsilon} \mathbb{L}_{\lambda}^{\omega}=\mathbf{L}_{\lambda}^{\omega} .
$$

Note that $\mathcal{L}_{\lambda}^{\omega \sharp}, \mathbb{L}_{\lambda}^{\omega}$ are naturally pure of weight zero.

Let $\mathcal{D} Z$ be the subcategory of $\mathcal{D}(Z)$ consisting of objects which are restrictions of objects in the $G \times \mathbf{T}^{2}$-equivariant derived category. Let $\mathcal{D}_{m}^{\mathbf{s}} Z$ be the subcategory of $\mathcal{D}_{m}(Z)$ consisting of objects which are restrictions of objects in the mixed $G \times \mathbf{T}^{2}$-equivariant derived category. Let $\mathcal{M} Z$ (resp. $\mathcal{M}_{m}^{\mathbf{A}} Z$ ) be the subcategory of $\mathcal{D}^{\mathbf{a}} Z$ (resp. $\mathcal{D}_{m}^{\mathbf{m}} Z$ ) consisting of objects which are perverse sheaves. We define $\mathcal{D} \preceq Z, \mathcal{D}^{\prec} Z, \mathcal{M} \preceq Z, \mathcal{M}^{\prec} Z, \mathcal{D}_{m}^{\prec} Z, \mathcal{D}_{m}^{\prec} Z$, $\mathcal{M}_{m}^{\prec} Z, \mathcal{M}_{m}^{\prec} Z, \mathcal{C}^{-} Z, \mathcal{C}_{0}^{\mathbf{c}} Z$ as in 2.14, by replacing (in 2.14) $Y$ by $Z$ and $\mathbf{L}_{\lambda}^{\dot{w}}$ by $\mathbb{L}_{\lambda}^{\dot{w}}$ (with $w \cdot \lambda$ required to be in $\widetilde{W}$ ). For $M \in \mathcal{C}_{0}^{\boldsymbol{A}} Z$ let $\underline{M}$ be the largest subobject of $M$ such that as an object of $\mathcal{C} Z$, we have $\underline{M} \in \mathcal{C}^{\mathbf{c}} Z$.

From 3.2(a) we see that, if $M \in \mathcal{M} Z$, then any composition factor of $M$ is of the form $\mathbb{L}_{\lambda}^{\dot{w}}$ for some $w \cdot \lambda \in \widetilde{W} \mathfrak{s}$. From the definitions we see that $M \mapsto \tilde{\epsilon} M$ is a functor $\mathcal{D}^{\mathbf{n}} Z \rightarrow \mathcal{D}^{\tilde{\mathcal{B}}^{2}}$ and also $\mathcal{D}_{m}^{\mathbf{m}} Z \rightarrow \mathcal{D}_{m}^{\mathbf{B}} \tilde{\mathcal{B}}^{2}$; moreover, it is a functor $\mathcal{M}^{\boldsymbol{\omega}} Z \rightarrow \mathcal{M}^{\mathbf{B}} \tilde{\mathcal{B}}^{2}$ and also $\mathcal{M}_{m}^{\mathbf{m}} Z \rightarrow \mathcal{M}_{m}^{\infty} \tilde{\mathcal{B}}^{2}$. From the definitions we see that for $M \in \mathcal{M} Z$ we have

(a) $M \in \mathcal{M} \preceq Z$ if and only if $\tilde{\epsilon} M \in \mathcal{M}^{\prec} \tilde{\mathcal{B}}^{2}$; we have $M \in \mathcal{M}^{\prec} Z$ if and only if $\tilde{\epsilon} M \in \mathcal{M}^{\prec} \tilde{\mathcal{B}}^{2}$.

Note that if $X \in \mathcal{D}(Z)$ and $j \in \mathbf{Z}$ then

$$
\left(\epsilon^{*} X\right)^{j+\rho}=\epsilon^{*}\left(X^{j}\right)[\rho] .
$$

Moreover, if $Y \in \mathcal{M}_{m}(Z)$ and $j^{\prime} \in \mathbf{Z}$ then

$$
g r_{j^{\prime}}(\tilde{\epsilon} Y)=\tilde{\epsilon}\left(g r_{j^{\prime}} Y\right)
$$

Let $\lambda \in \mathfrak{s}, w \in W_{\lambda}^{\prime}, \omega \in \kappa^{-1}(w)$. From 2.2(a) we deduce

$$
\left(\mathbb{L}_{\lambda}^{\omega}\right)^{\dagger}=\mathbb{L}_{\lambda^{-1}}^{\omega^{-1}}
$$


3.4. Let $r, f$ be integers such that $0 \leq f \leq r-3$. Let $\mathcal{Y}=\left\{\left(\left(x_{0} \mathbf{U}, x_{1} \mathbf{U}, \ldots, x_{r} \mathbf{U}\right), g\right) \in \tilde{\mathcal{B}}^{r+1} \times G ; g \in x_{f+3} \mathbf{U} x_{f}^{-1}, g \in x_{f+2} \mathbf{B} x_{f+1}^{-1}\right\}$

Define $\vartheta: \mathcal{Y} \rightarrow \tilde{\mathcal{B}}^{r+1}$ by

$$
\left(\left(x_{0} \mathbf{U}, x_{1} \mathbf{U}, \ldots, x_{r} \mathbf{U}\right), g\right) \mapsto\left(x_{0} \mathbf{U}, x_{1} \mathbf{U}, \ldots, x_{r} \mathbf{U}\right)
$$

For $y, y^{\prime} \in W$ let

$$
\tilde{\mathcal{B}}_{\left[y, y^{\prime}\right]}^{r+1}=\left\{\left(x_{0} \mathbf{U}, x_{1} \mathbf{U}, \ldots, x_{r} \mathbf{U}\right) \in \tilde{\mathcal{B}}^{r+1} ; x_{f}^{-1} x_{f+1} \in G_{y}, x_{f+2}^{-1} x_{f+3} \in G_{y^{\prime}}\right\} .
$$

We show:

(a) Let $\xi \in \tilde{\mathcal{B}}_{\left[y, y^{\prime}\right]}^{r+1}$. If $y y^{\prime} \neq 1$ then $\vartheta^{-1}(\xi)=\emptyset$. If $y y^{\prime}=1$ then $\vartheta^{-1}(\xi) \cong$ $\mathbf{k}^{\nu-|y|}$.

We set $\xi=\left(x_{0} \mathbf{U}, x_{1} \mathbf{U}, \ldots, x_{r} \mathbf{U}\right)$. If $\vartheta^{-1}(\xi) \neq \emptyset$ then $x_{f}^{-1} x_{f+1} \in G_{y}$, $x_{f+2}^{-1} x_{f+3} \in G_{y^{\prime}}$ and $\left(x_{f+3} \mathbf{U} x_{f}^{-1}\right) \cap\left(x_{f+2} \mathbf{B} x_{f+1}^{-1}\right) \neq \emptyset$. Hence for some $u \in \mathbf{U}, b \in \mathbf{B}$ we have

$$
u x_{f}^{-1} x_{f+1}=x_{f+3}^{-1} x_{f+2} b \in G_{y} \cap G_{y^{\prime-1}}
$$

so that $y y^{\prime}=1$. If we assume that $y y^{\prime}=1$, then $\vartheta^{-1}(\xi)$ can be identified with

$$
\left\{g \in G ; g \in x_{f+3} \mathbf{U} x_{f}^{-1}, g \in x_{f+2} \mathbf{B} x_{f+1}^{-1}\right\}
$$

hence with

$$
\left\{(u, b) \in \mathbf{U} \times \mathbf{B} ; u x_{f}^{-1} x_{f+1}=x_{f+3}^{-1} x_{f+2} b\right\} .
$$

We substitute $x_{f+3}^{-1} x_{f+2}=u_{0} \dot{y} t_{0} u_{0}^{\prime}, x_{f}^{-1} x_{f+1}=u_{1} \dot{y} t_{1} u_{1}^{\prime}$ where $u_{0}, u_{0}^{\prime}, u_{1}, u_{1}^{\prime}$ $\in \mathbf{U}, t_{0} \in \mathbf{T}$. Then $\vartheta^{-1}(\xi)$ is identified with $\left\{(u, b) \in \mathbf{U} \times \mathbf{B} ; u u_{1} \dot{y} t_{1} u_{1}^{\prime}=\right.$ $\left.u_{0} \dot{y} t_{0} u_{0}^{\prime} b\right\}$. The map $(u, b) \mapsto u_{0}^{-1} u u_{1}$ identifies this variety with $\mathbf{U} \cap$ $\dot{y} \mathbf{B} \dot{y}^{-1} \cong \mathbf{k}^{\nu-|y|}$. This proves (a).

Now $\mathbf{T}^{2}$ acts freely on $\mathcal{Y}$ by

$$
\begin{gathered}
\left(t_{1}, t_{2}\right):\left(\left(x_{0} \mathbf{U}, x_{1} \mathbf{U}, \ldots, x_{r} \mathbf{U}\right), g\right) \mapsto \\
\left(\left(x_{0} \mathbf{U}, x_{1} \mathbf{U}, \ldots, x_{f} \mathbf{U}, x_{f+1} t_{1} \mathbf{U}, x_{f+2} t_{2} \mathbf{U}, x_{f+3} \mathbf{U}, \ldots, x_{r} \mathbf{U}\right), g\right) .
\end{gathered}
$$


Let

$! \mathcal{Y}=\mathbf{T} \backslash\left\{\left(\left(x_{0} \mathbf{U}, x_{1} \mathbf{U}, \ldots, x_{r} \mathbf{U}\right), g\right) \in \tilde{\mathcal{B}}^{r+1} \times G ; g \in x_{f+3} \mathbf{U} x_{f}^{-1}, g \in x_{f+2} \mathbf{U} x_{f+1}^{-1}\right\}$

where $\mathbf{T}$ acts freely by

$$
\begin{gathered}
t:\left(\left(x_{0} \mathbf{U}, x_{1} \mathbf{U}, \ldots, x_{r} \mathbf{U}\right), g\right) \mapsto \\
\left(\left(x_{0} \mathbf{U}, x_{1} \mathbf{U}, \ldots, x_{f} \mathbf{U}, x_{f+1} t \mathbf{U}, x_{f+2} t \mathbf{U}, x_{f+3} \mathbf{U}, \ldots, x_{r} \mathbf{U}\right), g\right) .
\end{gathered}
$$

Clearly, the obvious map $\beta:{ }^{!} \mathcal{Y} \rightarrow \mathbf{T}^{2} \backslash \mathcal{Y}$ is an isomorphism. We define $!^{\prime}:{ }^{!} \mathcal{Y} \rightarrow Z$ by

$$
\left(\left(x_{0} \mathbf{U}, x_{1} \mathbf{U}, \ldots, x_{r} \mathbf{U}\right), g\right) \mapsto \epsilon\left(x_{f+1} \mathbf{U}, x_{f+2} \mathbf{U}\right)
$$

We define $\tau: \mathcal{Y} \rightarrow ! \mathcal{Y}$ as the composition of the obvious map $\mathcal{Y} \rightarrow \mathbf{T}^{2} \backslash \mathcal{Y}$ with $\beta^{-1}$. Let $\eta={ }^{!} \eta \tau: \mathcal{Y} \rightarrow Z$. We have

$$
\eta\left(\left(x_{0} \mathbf{U}, x_{1} \mathbf{U}, \ldots, x_{r} \mathbf{U}\right), g\right)=\epsilon\left(x_{f+1} t^{-1} \mathbf{U}, x_{f+2} t^{\prime-1} \mathbf{U}\right)
$$

where $t, t^{\prime}$ in $\mathbf{T}$ are such that $g \in x_{f+2} t^{\prime-1} \mathbf{U} t x_{f+1}^{-1}$.

3.5. Let $z \cdot \lambda \in \widetilde{W} \mathfrak{s}$. Let $P=\eta^{*} \mathcal{L}_{\lambda}^{\dot{z} \sharp}$. Let $p_{i j}: \tilde{\mathcal{B}}^{r+1} \rightarrow \tilde{\mathcal{B}}^{2}$ be the projection to the $i j$ coordinates. We have the following result:

(a) $\vartheta_{!} P \bumpeq\left\{p_{f, f+1}^{*} L_{\lambda}^{\dot{y}} \otimes p_{f+1, f+2}^{*} L_{\lambda}^{\dot{z} \sharp} \otimes p_{f+2, f+3}^{*} L_{y(\lambda)}^{\dot{y}^{-1}}\langle 2|y|-2 \nu\rangle ; y \in W\right\}$.

Define $e: \tilde{\mathcal{B}}^{r+1} \rightarrow \tilde{\mathcal{B}}^{4}$ by

$$
\left(x_{0} \mathbf{U}, x_{1} \mathbf{U}, \ldots, x_{r} \mathbf{U}\right) \mapsto\left(x_{f} \mathbf{U}, x_{f+1} \mathbf{U}, x_{f+2} \mathbf{U}, x_{f+3} \mathbf{U}\right)
$$

Then (a) is obtained by applying $e^{*}$ to the statement similar to (a) in which $\{0,1, \ldots, r\}$ is replaced by $\{f, f+1, f+2, f+3\}$. Thus it is enough to prove (a) in the special case where $r=3, f=0$. In the remainder of the proof we assume that $r=3, f=0$.

For any $y, y^{\prime}$ in $W$ let $\vartheta_{y, y^{\prime}}: \vartheta^{-1}\left(\tilde{\mathcal{B}}_{\left[y, y^{\prime}\right]}^{4}\right) \rightarrow \tilde{\mathcal{B}}^{4}$ be the restriction of $\vartheta$. Let $P^{y, y^{\prime}}$ be the restriction of $P$ to $\vartheta^{-1}\left(\tilde{\mathcal{B}}^{4}\right)_{\left[y, y^{\prime}\right]}$. Clearly, we have

$$
\vartheta_{!} P \approx\left\{\vartheta_{y, y^{\prime} !} P^{y, y^{\prime}} ;\left(y, y^{\prime}\right) \in W^{2}\right\}
$$


Since $\vartheta^{-1}\left(\tilde{\mathcal{B}}_{\left[y, y^{\prime}\right]}^{r+1}\right)=\emptyset$ when $y y^{\prime} \neq 1$, see $3.4(\mathrm{a})$, we deduce that

$$
\vartheta_{!} P \approx\left\{\vartheta_{y, y^{-1} !} P^{y, y^{-1}} ; y \in W\right\}
$$

Hence to prove (a) it is enough to show for any $y \in W$ that

$$
\vartheta_{y !} P_{y}=p_{01}^{*} L_{\lambda}^{\dot{y}} \otimes p_{12}^{*} L_{\lambda}^{\dot{z} \sharp} \otimes p_{23}^{*} L_{y(\lambda)}^{\dot{y}^{-1}}\langle 2|y|-2 \nu\rangle
$$

where we write $\vartheta_{y}, P_{y}$ instead of $\vartheta_{y, y^{-1}}, P^{y, y^{-1}}$. We have a cartesian diagram

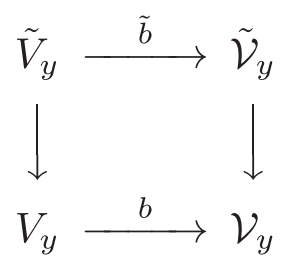

where

$$
\begin{gathered}
V_{y}=\left\{\left(x_{0} \mathbf{U}, x_{1} \mathbf{U}, x_{2} \mathbf{U}, x_{3} \mathbf{U}\right) \in \tilde{\mathcal{B}}^{4} ; x_{0}^{-1} x_{1} \in G_{y}, x_{1}^{-1} x_{2} \in G_{z}, x_{2}^{-1} x_{3} \in G_{y^{-1}}\right\}, \\
\mathcal{V}_{y}=\mathbf{T} \backslash\left\{\left(x_{0} \mathbf{U}, x_{1} \mathbf{U}, x_{2} \mathbf{U}, x_{3} \mathbf{U}\right) \in \tilde{\mathcal{B}}^{4} ; x_{0}^{-1} x_{1} \in G_{y}, x_{1}^{-1} x_{2} \in G_{z},\right. \\
\left.x_{2}^{-1} x_{3} \in G_{y^{-1}},\left(x_{0}^{-1} x_{1}\right)_{\dot{y}}=\left(x_{3}^{-1} x_{2}\right)_{\dot{y}}\right\}
\end{gathered}
$$

with $\mathbf{T}$ acting freely by simultaneous right multiplication on $x_{1}$ and $x_{2}$, $\tilde{V}_{y}=\vartheta^{-1}\left(V_{y}\right)$ and

$$
\begin{gathered}
\tilde{\mathcal{V}}_{y}=\mathbf{T} \backslash\left\{\left(\left(x_{0} \mathbf{U}, x_{1} \mathbf{U}, x_{2} \mathbf{U}, x_{3} \mathbf{U}\right), g\right) \in \tilde{\mathcal{B}}^{4} \times G ; x_{0}^{-1} x_{1} \in G_{y}, x_{1}^{-1} x_{2} \in G_{z},\right. \\
\left.x_{2}^{-1} x_{3} \in G_{y^{-1}}, g \in x_{3} \mathbf{U} x_{0}^{-1}, g \in x_{2} \mathbf{U} x_{1}^{-1}\right\} ;
\end{gathered}
$$

we have

$$
b\left(x_{0} \mathbf{U}, x_{1} \mathbf{U}, x_{2} \mathbf{U}, x_{3} \mathbf{U}\right)=\mathbf{T}-\text { orbit of }\left(x_{0} \mathbf{U}, x_{1} t \mathbf{U}, x_{2} t^{\prime} \mathbf{U}, x_{3} \mathbf{U}\right)
$$

where $t, t^{\prime}$ in $\mathbf{T}$ are such that $\left(x_{0}^{-1} x_{1} t\right)_{\dot{y}}=\left(x_{3}^{-1} x_{2} t^{\prime}\right)_{\dot{y}}$,

$$
\tilde{b}\left(\left(x_{0} \mathbf{U}, x_{1} \mathbf{U}, x_{2} \mathbf{U}, x_{3} \mathbf{U}\right), g\right)=\mathbf{T}-\text { orbit of }\left(\left(x_{0} \mathbf{U}, x_{1} t \mathbf{U}, x_{2} t^{\prime} \mathbf{U}, x_{3} \mathbf{U}\right), g\right)
$$

where $t, t^{\prime}$ in $\mathbf{T}$ are such that $g \in x_{2} t^{\prime} \mathbf{U} t^{-1} x_{1}^{-1}$; the vertical maps are the obvious ones. We also have a cartesian diagram 


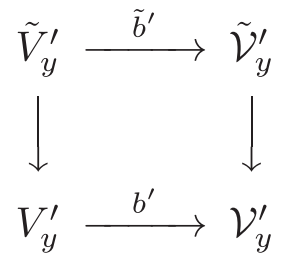

where $\tilde{V}_{y}^{\prime}, \tilde{\mathcal{V}}_{y}^{\prime}, V_{y}^{\prime}, \mathcal{V}_{y}^{\prime}$ are defined in the same way as $\tilde{V}_{y}, \tilde{\mathcal{V}}_{y}, V_{y}, \mathcal{V}_{y}$ but the condition $x_{1}^{-1} x_{2} \in G_{z}$ is replaced by the condition $x_{1}^{-1} x_{2} \in \bar{G}_{z}$; the maps $\tilde{b}^{\prime}, b^{\prime}$ are given by the same formulas as $\tilde{b}, b$; the vertical maps are the obvious ones.

Let $j: V_{y}^{\prime} \rightarrow \tilde{\mathcal{B}}^{4}$ be the inclusion. It is enough to show that

$$
j^{*} \vartheta_{y !} P_{y}=j^{*}\left(p_{01}^{*} L_{\lambda}^{\dot{y}} \otimes p_{12}^{*} L_{\lambda}^{\dot{z} \sharp} \otimes p_{23}^{*} L_{y(\lambda)}^{\dot{y}^{-1}}\right)\langle 2|y|-2 \nu\rangle .
$$

By definition, $\left.P\right|_{\tilde{V}_{y}^{\prime}}$ is the inverse image of $\mathcal{L}_{\lambda}^{\not \sharp}$ under the composition of $\tilde{b}^{\prime}$

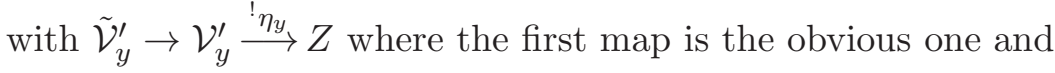

$$
{ }^{!} \eta_{y}\left(x_{0} \mathbf{U}, x_{1} \mathbf{U}, x_{2} \mathbf{U}, x_{3} \mathbf{U}\right)=\epsilon\left(x_{1} \mathbf{U}, x_{2} \mathbf{U}\right)
$$

Hence $\left.P\right|_{\tilde{V}_{y}^{\prime}}$ is the inverse image of $\mathcal{L}_{\lambda}^{\dot{z} \sharp}$ under the composition of $\eta_{y}:={ }^{!} \eta_{y} b^{\prime}$ with the obvious map $\vartheta_{y}^{\prime}: \tilde{V}_{y}^{\prime} \rightarrow V_{y}^{\prime}$. Since $\vartheta_{y}$ is an affine space bundle with fibres of dimension $\nu-|y|$, it follows that $j^{*} \vartheta_{y !} P_{y}=\eta_{y}^{*} \mathcal{L}_{\lambda}^{\dot{z}}\langle 2|y|-2 \nu\rangle$. Thus it is enough to show that

$$
\eta_{y}^{*} \mathcal{L}_{\lambda}^{\dot{z} \sharp}=j^{*}\left(p_{01}^{*} L_{\lambda}^{\dot{y}} \otimes p_{12}^{*} L_{\lambda}^{\dot{z} \sharp} \otimes p_{23}^{*} L_{y(\lambda)}^{\dot{y}^{-1}}\right) .
$$

Since $\eta_{y}$ is smooth as a map to $\bar{Z}_{z}$, we see that $\eta_{y}^{*} \mathcal{L}_{\lambda}^{\dot{z} \sharp}$ is the intersection cohomology complex of $V_{y}^{\prime}$ with coefficients in the local system $\eta_{y}^{*} \mathcal{L}_{\lambda}^{\dot{z}}$ on $V_{y}$. Now,

$$
j^{*}\left(p_{01}^{*} L_{\lambda}^{\dot{y}} \otimes p_{12}^{*} L_{\lambda}^{\dot{z} \sharp} \otimes p_{23}^{*} L_{y(\lambda)}^{\dot{y}^{-1}}\right)
$$

is the intersection cohomology complex of $V_{y}^{\prime}$ with coefficients in the local system

$$
j^{*}\left(p_{01}^{*} L_{\lambda}^{\dot{y}} \otimes p_{12}^{*} L_{\lambda}^{\dot{z}} \otimes p_{23}^{*} L_{y(\lambda)}^{\dot{y}^{-1}}\right)
$$

on $V_{y}$. It is then enough to show that these two local systems on $V_{y}$ are the 
same. One local system is $h^{*} L_{\lambda}$ with $h: V_{y} \rightarrow \mathbf{T}$ given by

$$
\left(x_{0} \mathbf{U}, x_{1} \mathbf{U}, x_{2} \mathbf{U}, x_{3} \mathbf{U}\right) \mapsto\left(t_{1} x_{1}^{-1} x_{2} t_{2}^{-1}\right)_{\dot{z}}
$$

where

$$
t_{1}=\left(x_{0}^{-1} x_{1}\right)_{\dot{y}} \in \mathbf{T}, t_{2}=\left(x_{3}^{-1} x_{2}\right)_{\dot{y}} \in \mathbf{T} .
$$

The other local system is $h^{\prime *} L_{\lambda}$ with $h^{\prime}: V_{y} \rightarrow \mathbf{T}$ given by

$$
\left(x_{0} \mathbf{U}, x_{1} \mathbf{U}, x_{2} \mathbf{U}, x_{3} \mathbf{U}\right) \mapsto t_{1}^{\prime} z\left(t_{2}^{\prime}\right)\left(z y^{-1}\right)\left(t_{3}^{\prime}\right)
$$

where

$$
t_{1}^{\prime}=\left(x_{0}^{-1} x_{1}\right)_{\dot{y}} \in \mathbf{T}, t_{2}^{\prime}=\left(x_{1}^{-1} x_{2}\right)_{\dot{z}} \in \mathbf{T}, t_{3}^{\prime}=\left(x_{2}^{-1} x_{3}\right)_{\dot{y}^{-1}} \in \mathbf{T} .
$$

It is enough to show that $h^{*} L_{\lambda}=h^{\prime *} L_{\lambda}$. Since the map $u: \mathbf{T} \rightarrow \mathbf{T}$, $t \mapsto z^{-1}(t)$ satisfies $u^{*} L_{\lambda}=L_{\lambda}$ (recall that $z(\lambda)=\lambda$ ), we have $h^{\prime *} L_{\lambda}=$ $h^{\prime *} u^{*} L_{\lambda}$ hence it is enough to show that $h(\xi)=\zeta^{-1}\left(h^{\prime}(\xi)\right)$ for any $\xi=$ $\left(x_{0} \mathbf{U}, x_{1} \mathbf{U}, x_{2} \mathbf{U}, x_{3} \mathbf{U}\right) \in V$, or that, if $t_{1}, t_{2}, t_{1}^{\prime}, t_{2}^{\prime}, t_{3}^{\prime}$ are associated to $\xi$ as above, then

$$
\left(t_{1} x_{1}^{-1} x_{2} t_{2}^{-1}\right)_{\dot{z}}=z^{-1}\left(t_{1}^{\prime} z\left(t_{2}^{\prime}\right)\left(z y^{-1}\right)\left(t_{3}^{\prime}\right)\right) .
$$

We have $t_{1}=t_{1}^{\prime}$ and $x_{3}^{-1} x_{2} \in \mathbf{U} \dot{y} t_{2} \mathbf{U}$ hence

$$
x_{2}^{-1} x_{3} \in \mathbf{U} t_{2}^{-1} \dot{y}^{-1} \mathbf{U}=\mathbf{U} \dot{y}^{-1} y\left(t_{2}^{-1}\right) \mathbf{U}
$$

so that $t_{3}^{\prime}=y\left(t_{2}^{-1}\right)$ and $t_{2}^{-1}=y^{-1}\left(t_{3}^{\prime}\right)$. We have

$$
t_{1} x_{1}^{-1} x_{2} t_{2}^{-1} \in t_{1} \mathbf{U} \dot{z} t_{2}^{\prime} \mathbf{U} t_{2}^{-1}=\mathbf{U} \dot{z} z^{-1}\left(t_{1}\right) t_{2}^{\prime} t_{2}^{-1} \mathbf{U}
$$

so that

$$
\left(t_{1} x_{1}^{-1} x_{2} t_{2}^{-1}\right) \dot{z}=z^{-1}\left(t_{1}\right) t_{2}^{\prime} t_{2}^{-1}=z^{-1}\left(t_{1}^{\prime}\right) t_{2}^{\prime} y^{-1}\left(t_{3}^{\prime}\right)
$$

as required. This completes the proof of (b) hence that of (a).

3.6. Let

$$
\begin{aligned}
\left(w_{1}, w_{2}, \ldots, w_{f}, w_{f+2}, w_{f+4}, \ldots, w_{r}\right) & \in W^{r-2} \\
\left(\lambda_{1}, \lambda_{2}, \ldots, \lambda_{f}, \lambda_{f+2}, \lambda_{f+4}, \ldots, \lambda_{r}\right) & \in \mathfrak{s}^{r-2}
\end{aligned}
$$

We set $z=w_{f+2}, \lambda=\lambda_{f+2}$. We assume that $z(\lambda)=\lambda$. Let $P$ be as in 3.5. Let $P^{\prime}=\otimes_{i \in[1, r]-\{f+1, f+2, f+3\}} p_{i-1, i}^{*} L_{\lambda_{i}}^{\dot{w}_{i} \sharp} \in \mathcal{D}_{m}\left(\tilde{\mathcal{B}}^{r+1}\right), \tilde{P}=P \otimes \vartheta^{*} P^{\prime} \in$ 
$\mathcal{D}_{m}(\mathcal{Y})$. For any $y \in W$ we set

$$
\begin{aligned}
& \mathbf{w}_{y}=\left(w_{1}, w_{2}, \ldots, w_{f}, y, w_{f+2}, y^{-1}, w_{f+4}, \ldots, w_{r}\right) \in W^{r}, \\
& \boldsymbol{\omega}_{y}=\left(\dot{w}_{1}, \dot{w}_{2}, \ldots, \dot{w}_{f}, \dot{y}, \dot{w}_{f+2}, \dot{y}^{-1}, \dot{w}_{f+4}, \ldots, \dot{w}_{r}\right), \\
& \boldsymbol{\lambda}_{y}=\left(\lambda_{1}, \lambda_{2}, \ldots, \lambda_{f}, \lambda_{f+2}, \lambda_{f+2}, y\left(\lambda_{f+2}\right), \lambda_{f+4}, \ldots, \lambda_{r}\right) \in \mathfrak{s}^{r} .
\end{aligned}
$$

We set $\Xi=\vartheta_{!} \tilde{P}$. We have the following result.

$$
\Xi \approx\left\{M_{\boldsymbol{\lambda}_{y}}^{\boldsymbol{\omega}_{y},[1, r]-\{f+1, f+3\}}\langle 2|y|-2 \nu\rangle ; y \in W\right\}
$$

in $\mathcal{D}_{m}\left(\tilde{\mathcal{B}}^{r+1}\right)$. This follows immediately from 3.5 (a) since $\Xi=P^{\prime} \otimes \vartheta_{!}(P)$.

3.7. We preserve the setup of 3.6. Let $\mathcal{S}=\sqcup_{\mathbf{w}^{\prime}} \tilde{\mathcal{O}}_{\mathbf{w}^{\prime}}^{\emptyset}$ where the union is over all $\mathbf{w}^{\prime}=\left(w_{1}^{\prime}, \ldots, w_{r}^{\prime}\right) \in W^{r}$ such that $w_{i}^{\prime}=w_{i}$ for $i \notin\{f+1, f+3\}$. This is a locally closed subvariety of $\tilde{\mathcal{B}}^{r+1}$. For $y \in W$ let $R_{y}$ be the restriction of $M_{\boldsymbol{\lambda}_{y}}^{\boldsymbol{\omega}_{y}, \emptyset}$ to $\tilde{\mathcal{O}}_{\mathbf{w}_{y}}^{\emptyset}$ extended by 0 on $\mathcal{S}-\tilde{\mathcal{O}}_{\mathbf{w}_{y}}^{\emptyset}$ (a constructible sheaf on $\mathcal{S}$ ). From the definitions we have

$$
\left.M_{\boldsymbol{\lambda}_{y},[1, r]-\{f+1, f+3\}}^{\boldsymbol{\omega}_{y}}\right|_{\mathcal{S}}=R_{y} .
$$

From 3.6(a) we deduce

$$
\left.\Xi\right|_{\mathcal{S}} \approx\left\{R_{y}\langle 2|y|-2 \nu\rangle ; y \in W\right\}
$$

We now restrict further to $\tilde{\mathcal{O}}_{\mathbf{w} y}^{\emptyset}($ for $y \in W)$; we obtain

$$
\left.\Xi\right|_{\tilde{\mathcal{O}}_{\mathbf{w} y}^{\emptyset}} ^{\emptyset} \approx\left\{\left.R_{y^{\prime}}\left\langle 2\left|y^{\prime}\right|-2 \nu\right\rangle\right|_{\tilde{\mathcal{O}}_{\mathbf{w} y}^{\emptyset}} ; y^{\prime} \in W\right\}
$$

In the right hand side we have $\left.R_{y^{\prime}}\left\langle 2\left|y^{\prime}\right|-2 \nu\right\rangle\right|_{\tilde{\mathcal{O}}_{\mathbf{w} y}^{\emptyset}}=0$ if $y^{\prime} \neq y$. It follows that $\left.\Xi\right|_{\tilde{\mathcal{O}}_{\mathbf{w}}} ^{\emptyset}=\left.R_{y}\langle 2|y|-2 \nu\rangle\right|_{\tilde{\mathcal{O}}_{\mathbf{w} y}^{\emptyset}}$. Since $\left.R_{y}\right|_{\tilde{\mathcal{O}}_{\mathbf{w} y}^{\emptyset}}$ is a local system we deduce for $y \in W^{\lambda}$ the following result.

(a) Let $h \in \mathbf{Z}$. If $h=2 \nu-2|y|$ then $\left.\mathcal{H}^{h} \Xi\right|_{\tilde{\mathcal{O}}_{\mathbf{w} y}^{\emptyset}}=\left.R_{y}\right|_{\tilde{\mathcal{O}}_{\mathbf{w} y}^{\emptyset}}(|y|-\nu)$. If $h \neq 2 \nu-2|y|$ then $\left.\mathcal{H}^{h} \Xi\right|_{\tilde{\mathcal{O}}_{\mathbf{w} y}^{\emptyset}}=0$.

3.8. We preserve the setup of 3.6. We set

$$
k=\left(\sum_{i \in[1, r]-\{f+1, f+3\}}\left|w_{i}\right|\right)+3 \nu+(r+1) \rho .
$$


For $y \in W$ we set

$$
\begin{aligned}
& K_{y}=M_{\boldsymbol{\lambda}_{y}}^{\boldsymbol{\omega}_{y},[1, r]-\{f+1, f+3\}}\left\langle\left|\mathbf{w}_{y}\right|+\nu+(r+1) \rho\right\rangle, \\
& \tilde{K}_{y}=M_{\boldsymbol{\lambda}_{y}}^{\boldsymbol{\omega}_{y},[1, r]}\left\langle\left|\mathbf{w}_{y}\right|+\nu+(r+1) \rho\right\rangle .
\end{aligned}
$$

From 3.6(a) we deduce:

$$
\Xi\langle k\rangle \bumpeq\left\{K_{y} ; y \in W\right\}
$$

We show:

(c) For any $j>0$ we have $(\Xi\langle k\rangle)^{j}=0$. Equivalently, $\Xi^{j}=0$ for any $j>k$.

Using (b) we see that it is enough to show that for any $y \in W$ we have $\left(K_{y}\right)^{j}=0$ for any $j>0$. Now $\tilde{K}_{y}$ is a (simple) perverse sheaf hence for any $i$ we have $\operatorname{dim} \operatorname{supp} \mathcal{H}^{i} \tilde{K}_{y} \leq-i$. Moreover $K_{y}$ is obtained by restricting $\tilde{K}_{y}$ to an open subset of its support and then extending the result (by zero) on the complement of this subset in $\tilde{\mathcal{B}}^{r+1}$. Hence supp $\mathcal{H}^{i} K_{y} \subset \operatorname{supp} \mathcal{H}^{i} \tilde{K}_{y}$ so that $\operatorname{dim} \operatorname{supp} \mathcal{H}^{i} K_{y} \leq-i$. Since this holds for any $i$ we see that $\left(K_{y}\right)^{j}=0$ for any $j>0$.

3.9. We preserve the notation of 3.6. We show:

(a) Let $j \in \mathbf{Z}$ and let $X$ be a composition factor of $\Xi^{j}$. Then $X \cong M_{\boldsymbol{\lambda}^{\prime}}^{\boldsymbol{\omega}^{\prime},[1, r]}\left\langle\left|\mathbf{w}^{\prime}\right|+\nu+(r+1) \rho\right\rangle$ for some

$$
\mathbf{w}^{\prime}=\left(w_{1}^{\prime}, w_{2}^{\prime}, \ldots, w_{r}^{\prime}\right) \in W^{r}, \lambda^{\prime}=\left(\lambda_{1}^{\prime}, \lambda_{2}^{\prime}, \ldots, \lambda_{r}^{\prime}\right) \in \mathfrak{s}^{r}
$$

such that $w_{i}^{\prime}=w_{i}, \lambda_{i}^{\prime}=\lambda_{i}$ for $i \in[1, r]-\{f+1, f+3\}$ and such that

$$
\lambda_{f+1}^{\prime}=w_{f+2}^{\prime}\left(\lambda_{f+2}^{\prime}\right), \lambda_{f+2}^{\prime}=w_{f+3}^{\prime}\left(\lambda_{f+3}^{\prime}\right) .
$$

Here $\boldsymbol{\omega}^{\prime}=\left(\dot{w}_{1}^{\prime}, \dot{w}_{2}^{\prime}, \ldots, \dot{w}_{r}^{\prime}\right)$.

From 3.6(a) we see that, for some $y \in W, X$ is a composition factor of

$$
\left(M_{\boldsymbol{\lambda}_{y}}^{\boldsymbol{\omega}_{y},[1, r]-\{f+1, f+3\}}\langle 2|y|-2 \nu\rangle\right)^{j} .
$$

Using this and 2.18(b) we see that $X$ is as required except that the equalities above for $\lambda_{f+1}^{\prime}, \lambda_{f+2}^{\prime}$ may not be satisfied. To see that they are in fact 
satisfied we note that

$$
\left(M_{\boldsymbol{\lambda}_{y}}^{\boldsymbol{\omega}_{y},[1, r]-\{f+1, f+3\}}\langle 2|y|-2 \nu\rangle\right)^{j}
$$

is equivariant for the $\mathbf{T}^{2}$-action

$$
\begin{gathered}
\left(t_{1}, t_{2}\right):\left(x_{0} \mathbf{U}, x_{1} \mathbf{U}, \ldots, x_{r} \mathbf{U}\right) \mapsto \\
\left(x_{0} \mathbf{U}, x_{1} \mathbf{U}, \ldots, x_{f} \mathbf{U}, x_{f+1} t_{1} \mathbf{U}, x_{f+2} t_{2} \mathbf{U}, x_{f+3} \mathbf{U}, \ldots, x_{r} \mathbf{U}\right)
\end{gathered}
$$

hence so are its composition factors and this implies that the equalities above for $\lambda_{f+1}^{\prime}, \lambda_{f+2}^{\prime}$ do hold.

3.10. From $3.8(\mathrm{c})$ we see that we have a distinguished triangle $\left(\Xi^{\prime}, \Xi, \Xi^{k}[-k]\right)$ where $\Xi^{\prime} \in \mathcal{D}_{m}\left(\tilde{\mathcal{B}}^{r+1}\right)$ satisfies $\left(\Xi^{\prime}\right)^{j}=0$ for all $j \geq k$. We show:

(a) Let $j \in \mathbf{Z}$ and let $K$ be one of $\Xi, \Xi^{j}, \Xi^{\prime}$. For any $\mathbf{w}^{\prime} \in W^{r}$ and any $h \in \mathbf{Z},\left.\mathcal{H}^{h} K\right|_{\tilde{\mathcal{O}}_{\mathbf{w}^{\prime}}^{\emptyset}}$ is a local system.

We prove (a) for $K=\Xi$. Using 3.6(a), we see that it is enough to show that $\left.\mathcal{H}^{h}\left(M_{\boldsymbol{\lambda}_{y}}^{\boldsymbol{\omega}_{y},[1, r]-\{f+1, f+3\}}\right)\right|_{\tilde{\mathcal{O}}_{\mathbf{w}^{\prime}}^{\emptyset}}$ is a local system for any $h$. This follows from the fact that $\theta^{*} M_{\boldsymbol{\lambda}_{y}}^{\boldsymbol{\omega}_{y},[1, r]-\{f+1, f+3\}}$ (see 2.15) is $\mathcal{G}$-equivariant.

We prove (a) for $K=\Xi^{j}$. Using 3.6(a), we see that it is enough to show that $\left.\mathcal{H}^{h}\left(\left(M_{\boldsymbol{\lambda}_{y}}^{\boldsymbol{\omega}_{y},[1, r]-\{f+1, f+3\}}\right)^{j}\right)\right|_{\tilde{\mathcal{O}}_{\mathbf{w}^{\prime}}^{\emptyset}}$ is a local system for any $h$ and any $j$. This again follows from the $\mathcal{G}$-equivariance statement in the previous paragraph.

Now (a) for $K=\Xi^{\prime}$ follows from (a) for $\Xi$ and $\Xi^{k}[-k]$ using the long exact sequence for cohomology sheaves of $\left(\Xi^{\prime}, \Xi, \Xi^{k}[-k]\right)$ restricted to $\tilde{\mathcal{O}}_{\mathbf{w}^{\prime}}^{\emptyset}$. (b) Let $\left(y, y^{\prime}\right) \in W^{2}, i=2 \nu-|y|-\left|y^{\prime}\right|$. Let

$$
\mathbf{w}_{y, y^{\prime}}=\left(w_{1}, w_{2}, \ldots, w_{f}, y, w_{f+2}, y^{\prime}, w_{f+3}, \ldots, w_{r}\right) \in W^{r}
$$

The induced homomorphism $\left.\left.\mathcal{H}^{i} \Xi\right|_{\tilde{\mathcal{O}}_{\mathbf{w}_{y, y^{\prime}}}^{\emptyset}} \rightarrow \mathcal{H}^{i-k}\left(\Xi^{k}\right)\right|_{\tilde{\mathcal{O}}_{\mathbf{w}_{y, y^{\prime}}}^{\emptyset}}$ is an isomorphism.

We have an exact sequence of constructible sheaves

$$
\left.\left.\left.\left.\mathcal{H}^{i} \Xi^{\prime}\right|_{\tilde{\mathcal{O}}_{\mathbf{w}_{y, y^{\prime}}}^{\emptyset}} \rightarrow \mathcal{H}^{i} \Xi\right|_{\tilde{\mathcal{O}}_{\mathbf{w}_{y, y^{\prime}}}^{\emptyset}} \rightarrow \mathcal{H}^{i-k}\left(\Xi^{k}\right)\right|_{\tilde{\mathcal{O}}_{\mathbf{w}_{y, y^{\prime}}}^{\emptyset}} \rightarrow \mathcal{H}^{i+1} \Xi^{\prime}\right|_{\tilde{\mathcal{O}}_{\mathbf{w}_{y, y^{\prime}}}^{\emptyset}}
$$


Hence it is enough to show that $\left.\mathcal{H}^{i^{\prime}} \Xi^{\prime}\right|_{\tilde{\mathcal{O}}_{\mathbf{w}_{y, y^{\prime}}}^{\emptyset}}=0$ if $i^{\prime} \geq i$. Assume that

$$
\left.\mathcal{H}^{i^{\prime}} \Xi^{\prime}\right|_{\tilde{\mathcal{O}}_{\mathbf{w}_{y, y^{\prime}}}^{\emptyset}} \neq 0
$$

for some $i^{\prime} \geq i$. Since $\left.\mathcal{H}^{i^{\prime}} \Xi^{\prime}\right|_{\tilde{\mathcal{O}}_{\mathbf{w}_{y, y^{\prime}}}^{\emptyset}}$ is a local system (see (a)), we deduce that $\tilde{\mathcal{O}}_{\mathbf{w}_{y, y^{\prime}}}^{\emptyset}$ is contained in $\operatorname{supp}\left(\mathcal{H}^{i^{\prime} \Xi^{\prime}}\right)$. We have $\left(\Xi^{\prime}[k-1]\right)^{j}=0$ for any $j>0$ hence $\operatorname{dim} \operatorname{supp}\left(\mathcal{H}^{i^{\prime \prime}} \Xi^{\prime}[k-1]\right) \leq-i^{\prime \prime}$ for any $i^{\prime \prime}$. Taking $i^{\prime \prime}=i^{\prime}-k+1$ we deduce that

$$
\operatorname{dim} \tilde{\mathcal{O}}_{\mathbf{w}_{y, y^{\prime}}}^{\emptyset} \leq \operatorname{dim} \operatorname{supp}\left(\mathcal{H}^{i^{\prime}} \Xi^{\prime}\right) \leq-i^{\prime}+k-1 \leq-i+k-1
$$

hence

$$
\left|\mathbf{w}_{y, y^{\prime}}\right|+\nu+(r+1) \rho \leq-i+k-1 \text {. }
$$

We have $\left|\mathbf{w}_{y, y^{\prime}}\right|+\nu+(r+1) \rho=-i+k$ hence $-i+k \leq-i+k-1$, contradiction. This proves (b).

3.11. For $\left(y, y^{\prime}\right) \in W^{2}$ we set

$$
\begin{aligned}
\boldsymbol{\omega}_{y, y^{\prime}} & =\left(\dot{w}_{1}, \dot{w}_{2}, \ldots, \dot{w}_{f}, \dot{y}, \dot{w}_{f+2},\left(\left(y^{\prime-1}\right)^{\prime}\right)^{-1}, \dot{w}_{f+3}, \ldots, \dot{w}_{r}\right) \in W^{r}, \\
\lambda_{y, y^{\prime}} & =\left(\lambda_{1}, \lambda_{2}, \ldots, \lambda_{f}, \lambda_{f+2}, \lambda_{f+2}, y^{\prime-1}\left(\lambda_{f+2}\right), \lambda_{f+4}, \ldots, \lambda_{r}\right) \in \mathfrak{s}^{r}, \\
K_{y, y^{\prime}} & =M_{\lambda_{y, y^{\prime}}}^{\omega_{y, y^{\prime}}, \emptyset}\left\langle\left|\mathbf{w}_{y, y^{\prime}}\right|+\nu+(r+1) \rho\right\rangle \in \mathcal{M}_{m}\left(\tilde{\mathcal{B}}^{r+1}\right), \\
\tilde{K}_{y, y^{\prime}} & =M_{\lambda_{y, y^{\prime}}}^{\omega_{y,[1, r]}}\left\langle\left|\mathbf{w}_{y, y^{\prime}}\right|+\nu+(r+1) \rho\right\rangle \in \mathcal{M}_{m}\left(\tilde{\mathcal{B}}^{r+1}\right) .
\end{aligned}
$$

Note that when $y=y^{\prime}, \mathbf{w}_{y, y^{\prime}}, \boldsymbol{\omega}_{y, y^{\prime}}, \boldsymbol{\lambda}_{y, y^{\prime}}, K_{y, y^{\prime}}, \tilde{K}_{y, y^{\prime}}$ become $\mathbf{w}_{y}, \boldsymbol{\omega}_{y}, \boldsymbol{\lambda}_{y}$ (see 3.6) and $K_{y}, \tilde{K}_{y}$ (see 3.8 ). We show that we have canonically

$$
\operatorname{gr}_{0}\left(\Xi^{k}(k / 2)\right)=\oplus_{y \in W} \tilde{K}_{y}
$$

Since $\operatorname{gr}_{0}\left(\Xi^{k}(k / 2)\right)$ is a semisimple perverse sheaf of pure weight zero, it is a direct sum of simple perverse sheaves, necessarily of the form described in 3.9 (a). Thus we have canonically

$$
g r_{0}\left(\Xi^{k}(k / 2)\right)=\oplus_{\left(y, y^{\prime}\right) \in W^{2}} V_{y, y^{\prime}} \otimes \tilde{K}_{y, y^{\prime}}
$$

where $V_{y, y^{\prime}}$ are mixed $\overline{\mathbf{Q}}_{l^{-}}$vector spaces of pure weight 0 . By $[1,5.1 .14], \Xi$ is 
mixed of weight $\leq 0$ hence $\Xi^{k}(k / 2)$ is mixed of weight $\leq 0$. Hence we have an exact sequence in $\mathcal{M}_{m}\left(\tilde{\mathcal{B}}^{r+1}\right)$ :

$$
0 \rightarrow \mathcal{W}^{-1}\left(\Xi^{k}(k / 2)\right) \rightarrow \Xi^{k}(k / 2) \rightarrow g r_{0}\left(\Xi^{k}(k / 2)\right) \rightarrow 0
$$

that is,

$$
0 \rightarrow \mathcal{W}^{-1}\left(\Xi^{k}(k / 2)\right) \rightarrow \Xi^{k}(k / 2) \rightarrow \oplus_{\left(y, y^{\prime}\right) \in W^{2}} V_{y, y^{\prime}} \otimes \tilde{K}_{y, y^{\prime}} \rightarrow 0
$$

(Here $\mathcal{W}^{-1}(?)$ denotes the part of weight $\leq-1$ of a mixed perverse sheaf.) Hence for any $\left(\tilde{y}, \tilde{y}^{\prime}\right) \in W^{2}$ we have an exact sequence of (mixed) cohomology sheaves restricted to $\tilde{\mathcal{O}}_{\mathbf{w}_{\tilde{y}, \tilde{y}^{\prime}}^{\emptyset}}^{\emptyset}\left(\right.$ where $\left.h=2 \nu-|\tilde{y}|-\left|\tilde{y}^{\prime}\right|-k\right)$ :

$$
\text { (b) } \begin{aligned}
\mathcal{H}^{h}\left(\mathcal{W}^{-1}\left(\Xi^{k}(k / 2)\right)\right) & \stackrel{\alpha}{\rightarrow} \mathcal{H}^{h}\left(\Xi^{k}(k / 2)\right) \rightarrow \oplus_{\left(y, y^{\prime}\right) \in W^{2}} V_{y, y^{\prime}} \otimes \mathcal{H}^{h}\left(\tilde{K}_{y, y^{\prime}}\right) \\
& \rightarrow \mathcal{H}^{h+1}\left(\mathcal{W}^{-1}\left(\Xi^{k}(k / 2)\right)\right) .
\end{aligned}
$$

Moreover, by $3.10(\mathrm{~b})$, we have an equality of local systems on $\tilde{\mathcal{O}}_{\mathbf{w}_{\tilde{y}, \tilde{y}^{\prime}}^{\emptyset}}^{\emptyset}$ :

$$
\mathcal{H}^{h}\left(\Xi^{k}(k / 2)\right)=\mathcal{H}^{h+k}(\Xi(k / 2))=\mathcal{H}^{2 \nu-|y|-\left|y^{\prime}\right|}(\Xi(k / 2))
$$

and this is $R_{\tilde{y}}(k / 2+|\tilde{y}|-\nu)$ if $\tilde{y} \tilde{y}^{\prime}=1$ (see $\left.3.7(\mathrm{a})\right)$ and is 0 if $\tilde{y} \tilde{y}^{\prime} \neq 1$ (see $3.4(\mathrm{a}))$ hence is pure of weight $-k-|\tilde{y}|-\left|\tilde{y}^{\prime}\right|+\nu=h$. On the other hand, $\mathcal{H}^{h}\left(\mathcal{W}^{-1}\left(\Xi^{k}(k / 2)\right)\right)$ is mixed of weight $\leq h-1$; it follows that $\alpha$ in (b) must be zero.

Assume that $\mathcal{H}^{h}\left(\tilde{K}_{y, y^{\prime}}\right)$ is nonzero on $\tilde{\mathcal{O}}_{\mathbf{w}_{\tilde{y}, \tilde{y}^{\prime}}}^{\emptyset}$. Then, by $3.10(\mathrm{a}), \tilde{\mathcal{O}}_{\mathbf{w}_{\tilde{y}, \tilde{y}^{\prime}}^{\emptyset}}^{\emptyset}$ is contained in $\operatorname{supp} \mathcal{H}^{h}\left(\tilde{K}_{y, y^{\prime}}\right)$ which has dimension $\leq-h$ (resp. $<-h$ if $\left.\left(y, y^{\prime}\right) \neq\left(\tilde{y}, \tilde{y}^{\prime}\right)\right)$; hence $-h=\operatorname{dim} \tilde{\mathcal{O}}_{\mathbf{w}_{\tilde{y}, \tilde{y}^{\prime}}^{\emptyset}}$ is $\leq-h$ (resp. $\left.<-h\right)$; we see that we must have $\left(y, y^{\prime}\right)=\left(\tilde{y}, \tilde{y}^{\prime}\right)$ and we have $\mathcal{H}^{h}\left(\tilde{K}_{y, y^{\prime}}\right)=\mathcal{H}^{h}\left(K_{y, y^{\prime}}\right)$ on $\tilde{\mathcal{O}}_{\mathbf{w}_{\tilde{y}, \tilde{y}}}^{\emptyset}$.

Assume that $\mathcal{H}^{h+1}\left(\mathcal{W}^{-1}\left(\Xi^{k}(k / 2)\right)\right)$ is not identically 0 on $\tilde{\mathcal{O}}_{\mathbf{w}_{\tilde{y}, \tilde{y}}}^{\emptyset}$. Then, by $3.10(\mathrm{a}), \tilde{\mathcal{O}}_{\mathbf{w}_{\tilde{y}, \tilde{y}^{\prime}}}^{\emptyset}$ is contained in $\operatorname{supp} \mathcal{H}^{h+1}\left(\mathcal{W}^{-1}\left(\Xi^{k}(k / 2)\right)\right)$ which has dimension $\leq-h-1$; hence $-h=\operatorname{dim} \tilde{\mathcal{O}}_{\mathbf{w}_{\tilde{y}, \tilde{y}^{\prime}}}^{\emptyset} \leq-h-1$, a contradiction. We see that (b) becomes an isomorphism of local systems on $\tilde{\mathcal{O}}_{\mathbf{w}_{\tilde{y}, \tilde{y}^{\prime}}}^{\emptyset}$ :

$$
\begin{aligned}
& 0=V_{\tilde{y}, \tilde{y}^{\prime}} \otimes K_{\tilde{y}, \tilde{y}^{\prime}} \text { if } \tilde{y} \tilde{y}^{\prime} \neq 1, \\
& R_{\tilde{y}}(-h / 2) \stackrel{\sim}{\rightarrow} V_{\tilde{y}, \tilde{y}^{\prime}} \otimes \mathcal{H}^{h}\left(K_{\tilde{y}, \tilde{y}^{\prime}}\right) \text { if } \tilde{y} \tilde{y}^{\prime}=1
\end{aligned}
$$


When $\tilde{y} \tilde{y}^{\prime}=1$ we have $\mathcal{H}^{h}\left(K_{\tilde{y}, \tilde{y}^{\prime}}\right)=R_{\tilde{y}}(-h / 2)$ as local systems on $\tilde{\mathcal{O}}_{\mathbf{w}_{\tilde{y}, \tilde{y}^{\prime}}}^{\emptyset}$. It follows that $V_{\tilde{y}, \tilde{y}^{\prime}}$ is $\overline{\mathbf{Q}}_{l}$ if $\tilde{y} \tilde{y}^{\prime}=1$ and is 0 if $\tilde{y} \tilde{y}^{\prime} \neq 1$. This proves (a).

3.12. Let $h \in[1, r]$. Let ${ }_{h} \mathcal{D} \preceq \tilde{\mathcal{B}}^{r+1}$ (resp. ${ }_{h} \mathcal{D}^{\prec} \tilde{\mathcal{B}}^{r+1}$ ) be the subcategory of $\mathcal{D} \tilde{\mathcal{B}}^{r+1}$ consisting of objects $K$ such that for any $j \in \mathbf{Z}$, any composition factor of $K^{j}$ is of the form $M_{\boldsymbol{\lambda}}^{\boldsymbol{\omega},[1, r]}\langle|\mathbf{w}|+\nu+(r+1) \rho\rangle$ for some $\mathbf{w}=\left(w_{1}, \ldots, w_{r}\right) \in W^{r}, \boldsymbol{\lambda}=\left(\lambda_{1}, \lambda_{2}, \ldots, \lambda_{r}\right) \in \mathfrak{s}^{r}$ such that $w_{h} \cdot \lambda_{h} \preceq \mathbf{c}$ (resp. $\left.w_{h} \cdot \lambda_{h} \prec \mathbf{c}\right)$. (Here $\boldsymbol{\omega}=\left(\dot{w}_{1}, \dot{w}_{2}, \ldots, \dot{w}_{r}\right)$ ) $)$ Let ${ }_{h} \mathcal{M} \preceq \tilde{\mathcal{B}}^{r+1}$ (resp. ${ }_{h} \mathcal{M}^{\prec} \tilde{\mathcal{B}}^{r+1}$ ) be the subcategory of ${ }_{h} \mathcal{D} \preceq \tilde{\mathcal{B}}^{r+1}$ (resp. ${ }_{h} \mathcal{D}^{\prec} \tilde{\mathcal{B}}^{r+1}$ ) consisting of perverse sheaves.

If $K \in \mathcal{M}_{m}\left(\tilde{\mathcal{B}}^{r+1}\right)$ is pure of weight 0 and is also in ${ }_{h} \mathcal{D} \preceq \tilde{\mathcal{B}}^{r+1}$ we denote by $\underline{K}$ the sum of all simple subobjects of $K$ (without mixed structure) which are not in ${ }_{h} \mathcal{D}^{\prec} \tilde{\mathcal{B}}^{r+1}$.

3.13. Let $Z \stackrel{\eta}{\leftarrow} \mathcal{Y} \stackrel{\vartheta}{\rightarrow} \tilde{\mathcal{B}}^{4}$ be as in 3.4 with $r=3, f=0$. We define $\mathfrak{b}: \mathcal{D}(Z) \rightarrow$ $\mathcal{D}\left(\tilde{\mathcal{B}}^{2}\right)$ and $\mathfrak{b}: \mathcal{D}_{m}(Z) \rightarrow \mathcal{D}_{m}\left(\tilde{\mathcal{B}}^{2}\right)$ by

$$
\mathfrak{b}(L)=p_{03 !} \vartheta_{!} \eta^{*} L
$$

We show:

(a) If $L \in \mathcal{D} \preceq(Z)$ then $\mathfrak{b}(L) \in \mathcal{D} \preceq \tilde{\mathcal{B}}^{2}$.

(b) If $L \in \mathcal{D}^{\prec}(Z)$ then $\mathfrak{b}(L) \in \mathcal{D}^{\prec} \tilde{\mathcal{B}}^{2}$.

(c) If $L \in \mathcal{M}(Z)$ and $h>5 \rho+2 \nu+2 a$ then $(\mathfrak{b}(L))^{h} \in \mathcal{M}^{\prec} \tilde{\mathcal{B}}^{2}$.

We can assume that $L=\mathbb{L}_{\lambda}^{\dot{z}}$ where $z \cdot \lambda \in \widetilde{W} \mathfrak{s}, z \cdot \lambda \preceq \mathbf{c}$. Applying 3.5(a) with $P=\eta^{*} \mathcal{L}_{\lambda}^{i \sharp}$ we see that

$$
\mathfrak{b}\left(\mathcal{L}_{\lambda}^{\dot{z} \sharp}\right) \approx\left\{L_{\lambda, \lambda, y(\lambda)}^{\dot{y}, \dot{z}, \dot{y}^{-1},\{2\}}\langle-|z|-2 \nu\rangle ; y \in W\right\},
$$

hence

$$
\mathfrak{b}\left(\mathbb{L}_{\lambda}^{\dot{z} \sharp}\right) \approx\left\{L_{\lambda, \lambda, y(\lambda)}^{\dot{y}, \dot{z}, \dot{y}^{-1},\{2\}}\langle-\nu+\rho\rangle ; y \in W\right\} .
$$

To prove (a) it is enough to show that for any $y \in W$ we have

$$
L_{\lambda, \lambda, y(\lambda)}^{\dot{y}, \dot{z}, \dot{y}^{-1},\{2\}} \in \mathcal{D}^{\preceq} \tilde{\mathcal{B}}^{2} .
$$


When $z \cdot \lambda \in \mathbf{c}$ this follows from $2.10(\mathrm{a})$. When $z \cdot \lambda \prec \mathbf{c}$ this again follows from 2.10(a), applied to the two-sided cell containing $z \cdot \lambda$ instead of $\mathbf{c}$. The same argument proves (b). To prove (c) we can assume that $z \cdot \lambda \in \mathbf{c}$; it is enough to prove that for any $y \in W$ we have

$$
\left(L_{\lambda, \lambda, y(\lambda)}^{\dot{y}, \dot{z}, \dot{y}^{-1},\{2\}}\langle-\nu+\rho\rangle\right)^{h} \in \mathcal{M}^{\prec} \tilde{\mathcal{B}}^{2}
$$

if $h>5 \rho+2 \nu+2 a$ or that $\left(L_{\lambda, \lambda, y(\lambda)}^{\dot{y}, \dot{z}, \dot{y}^{-1},\{2\}}\right)^{j} \in \mathcal{M}^{\prec} \tilde{\mathcal{B}}^{2}$ if $j>6 \rho+\nu+2 a$. This follows from 2.20(a). This completes the proof of (a), (b), (c).

We define $\underline{\mathfrak{b}}: \mathcal{C}_{0}^{\mathbf{c}}(Z) \rightarrow \mathcal{C}_{0}^{\mathbf{c}}\left(\tilde{\mathcal{B}}^{2}\right)$ by

$$
\underline{\mathfrak{b}}(L)=\underline{g r_{5 \rho+2 \nu+2 a}\left((\mathfrak{b}(L))^{5 \rho+2 \nu+2 a}\right)}((5 \rho+2 \nu+2 a) / 2) .
$$

We show:

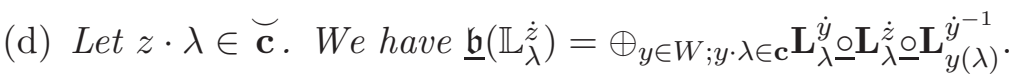

We shall apply [24, 1.12] with $\Phi: \mathcal{D}_{m}\left(Y_{1}\right) \rightarrow \mathcal{D}_{m}\left(Y_{2}\right)$ replaced by $p_{03 !}:$ $\mathcal{D}_{m}\left(\tilde{\mathcal{B}}^{4}\right) \rightarrow \mathcal{D}_{m}\left(\tilde{\mathcal{B}}^{2}\right)$ and with $\mathcal{D} \preceq\left(Y_{1}\right), \mathcal{D} \preceq\left(Y_{2}\right)$ replaced by ${ }_{2} \mathcal{D} \preceq\left(\tilde{\mathcal{B}}^{2}\right),{ }_{2} \mathcal{D} \preceq\left(\tilde{\mathcal{B}}^{4}\right)$, see 3.12. We shall take $\mathbf{X}$ in loc.cit. equal to $\vartheta_{!} \eta^{*} \mathbb{L}_{\lambda}^{\dot{z}}$. The conditions of loc.cit. are satisfied: those concerning $\mathbf{X}$ are satisfied with $c^{\prime}=2 \nu+3 \rho$. (For $h>|z|+3 \nu+4 \rho$ we have $\Xi^{h}=0$ that is $(\mathbf{X}[-|z|-\nu-\rho])^{h}=0$, with $\Xi$ as in 3.8(c). Hence if $j>2 \nu+3 \rho$ we have $\mathbf{X}^{j}=0$.) The conditions concerning $p_{03 \text { ! }}$ are satisfied with $c=2 \rho+2 a$. (This follows from 2.20(a).) Since $\mathfrak{b}\left(\mathbb{L}_{\dot{\lambda}}^{\dot{z}}\right)=p_{03 !} \mathbf{X}$ and $c+c^{\prime}=5 \rho+2 \nu+2 a$, we see that

$$
\left.\left.\left.\underline{\mathfrak{b}}\left(\mathbb{L}_{\lambda}^{\dot{z}}\right)=\underline{g r_{2 \rho+2 a}\left(p _ { 0 3 ! } \left(\left(g r_{2 \nu+3 \rho}\left(\left(\vartheta_{!} \eta^{*} \mathbb{L}_{\lambda}^{\dot{z}}\right)^{2 \nu+3 \rho}\right)\right.\right.\right.}((2 \nu+3 \rho) / 2)\right)\right)^{2 \rho+2 a}\right)(\rho+a) .
$$

Using 3.11(a) we see that (with $\Xi$ as in $3.11(\mathrm{a})$ and $k=|z|+3 \nu+4 \rho$ ) we have

$$
\begin{aligned}
& g r_{2 \nu+3 \rho}\left(\left(\vartheta_{!} \eta^{*} \mathbb{L}_{\lambda}^{\dot{z}}\right)^{2 \nu+3 \rho}\right)((2 \nu+3 \rho) / 2) \\
& =\frac{g r_{2 \nu+3 \rho}\left((\Xi\langle|z|+\nu+\rho\rangle)^{2 \nu+3 \rho}\right)}{}((2 \nu+3 \rho) / 2) \\
& =\underline{g r_{0}\left(\Xi^{k}(k / 2)\right.}=\oplus_{y \in W} M_{\lambda, \lambda, y(\lambda)}^{\dot{y}, \dot{z}, \dot{y}^{-1},[1,3]}\langle 2|y|+|z|+\nu+4 \rho\rangle .
\end{aligned}
$$

Hence

$$
\underline{\mathfrak{b}}\left(\mathbb{L}_{\lambda}^{\dot{z}}\right)=\underline{g r_{2 \rho+2 a}\left(\oplus_{y \in W}\left(p_{03 !} M_{\lambda, \lambda, y(\lambda)}^{\dot{y}, \dot{z}, \dot{y}^{-1},[1,3]}\langle 2|y|+|z|+\nu+4 \rho\rangle\right)^{2 \rho+2 a}\right)(\rho+a)}
$$




$$
=\underline{\operatorname{gr}_{2 \rho+2 a}\left(\oplus_{y \in W}\left(L_{\lambda, \lambda, y(\lambda)}^{\dot{y}, \dot{z}, \dot{y}^{-1},[1,3]}\right)^{6 \rho+\nu+2 a}((\nu+4 \rho) / 2)\right)(\rho+a) .}
$$

Using 2.26(a) we see that in the last direct sum the contribution of $y \in W$ is 0 unless $y \cdot \lambda \in \mathbf{c}$. For the terms corresponding to $y$ such that $y \cdot \lambda \in \mathbf{c}$, we may apply 2.24(a). Now (d) follows.

3.14. Let $Z \stackrel{!}{\longleftarrow} ! \mathcal{Y}$ be as in 3.4 with $r=3, f=0$. Let $!^{4}$ be the space of orbits of the free $\mathbf{T}^{2}$-action on $\tilde{\mathcal{B}}^{4}$ given by

$$
\left(t_{1}, t_{2}\right):\left(x_{0} \mathbf{U}, x_{1} \mathbf{U}, x_{2} \mathbf{U}, x_{3} \mathbf{U}\right) \mapsto\left(x_{0} \mathbf{U}, x_{1} t_{1} \mathbf{U}, x_{2} t_{2} \mathbf{U}, x_{3} \mathbf{U}\right)
$$

let $! \vartheta: ! \mathcal{Y} \rightarrow{ }^{!} \tilde{\mathcal{B}}^{4}$ be the map induced by $\vartheta$. We define $\mathfrak{b}^{\prime}: \mathcal{D}(Z) \rightarrow \mathcal{D}\left(\tilde{\mathcal{B}}^{2}\right)$ and $\mathfrak{b}^{\prime}: \mathcal{D}_{m}(Z) \rightarrow \mathcal{D}_{m}\left(\tilde{\mathcal{B}}^{2}\right)$ by

$$
\mathfrak{b}^{\prime}(L)=p_{03 !} ! \vartheta^{!} ! \eta^{*} L
$$

(The map $! \tilde{\mathcal{B}}^{4} \rightarrow \tilde{\mathcal{B}}^{2}$ induced by $p_{03}: \tilde{\mathcal{B}}^{4} \rightarrow \tilde{\mathcal{B}}^{2}$ is denoted again by $p_{03}$. ) Let $\tau: \mathcal{Y} \rightarrow ! \mathcal{Y}$ be as in 3.4 (it is a principal $T^{2}$-bundle). We have the following results.

(a) If $L \in \mathcal{D} \preceq(Z)$ then $\mathfrak{b}^{\prime}(L) \in \mathcal{D} \preceq \tilde{\mathcal{B}}^{2}$.

(b) If $L \in \mathcal{D}^{\prec}(Z)$ then $\mathfrak{b}^{\prime}(L) \in \mathcal{D}^{\prec} \tilde{\mathcal{B}}^{2}$.

(c) If $L \in \mathcal{M}(Z)$ and $h>\rho+2 \nu+2 a$ then $\left(\mathfrak{b}^{\prime}(L)\right)^{h} \in \mathcal{M}^{\prec} \tilde{\mathcal{B}}^{2}$.

We can assume that $L=\mathbb{L}_{\lambda}^{\dot{z}}$ where $z \cdot \lambda \in \breve{W} \mathfrak{s}, z \cdot \lambda \preceq$ c. A variant of the proof of 3.5(a) gives:

$$
\mathfrak{b}^{\prime}\left(\mathcal{L}_{\lambda}^{\dot{z} \sharp}\right) \approx\left\{{ }^{\prime} L_{\lambda, \lambda, y(\lambda)}^{\dot{y}, \dot{z}, \dot{y}^{-1},\{2\}}\langle-|z|-2 \nu\rangle ; y \in W\right\},
$$

hence

$$
\mathfrak{b}^{\prime}\left(\mathbb{L}_{\lambda}^{\dot{z} \sharp}\right) \approx\left\{L_{\lambda, \lambda, y(\lambda)}^{\dot{y}, \dot{z}, \dot{y}^{-1},\{2\}}\langle-\nu+\rho\rangle ; y \in W\right\} .
$$

To prove (a) it is enough to show that for any $y \in W$ we have

$$
{ }^{\prime} L_{\lambda, \lambda, y(\lambda)}^{\dot{y}, \dot{z}, \dot{y}^{-1},\{2\}} \in \mathcal{D}^{\preceq} \tilde{\mathcal{B}}^{2} .
$$

When $z \cdot \lambda \in \mathbf{c}$ this follows from 2.10(c). When $z \cdot \lambda \prec \mathbf{c}$ this again follows from 2.10(c), applied to the two-sided cell containing $z \cdot \lambda$ instead of $\mathbf{c}$. The 
same argument proves (b). To prove (c) we can assume that $z \cdot \lambda \in \mathbf{c}$; it is enough to prove that for any $y \in W$ we have

$$
\left({ }^{\prime} L_{\lambda, \lambda, y(\lambda)}^{\dot{y}, \dot{z}, \dot{y}^{-1},\{2\}}\langle-\nu+\rho\rangle\right)^{h} \in \mathcal{M}^{\prec} \tilde{\mathcal{B}}^{2}
$$

if $h>\rho+2 \nu+2 a$ or that $\left({ }^{\prime} L_{\lambda, \lambda, y(\lambda)}^{\dot{y}, \dot{z}, \dot{y}^{-1}},\{2\}\right){ }^{j} \in \mathcal{M}^{\prec} \tilde{\mathcal{B}}^{2}$ if $j>2 \rho+\nu+2 a$. This follows from 2.20(c). This completes the proof of (a), (b), (c).

We define $\underline{\mathfrak{b}^{\prime}}: \mathcal{C}_{0}^{\mathbf{c}}(Z) \rightarrow \mathcal{C}_{0}^{\mathbf{c}}\left(\tilde{\mathcal{B}}^{2}\right)$ by

$$
\underline{\mathfrak{b}}^{\prime}(L)=\underline{g r_{\rho+2 \nu+2 a}\left(\left(\mathfrak{b}^{\prime}(L)\right)^{\rho+2 \nu+2 a}\right)}((\rho+2 \nu+2 a) / 2) .
$$

In the remainder of this subsection we fix $z \cdot \lambda \in \breve{\mathbf{c}}$ and we set $L=\mathbb{L}_{\lambda}^{\dot{z}}$. We show:

(d) We have canonically $\underline{\mathfrak{b}}^{\prime}(L)=\underline{\mathfrak{b}}(L)$.

The method of proof is similar to that of $2.22(\mathrm{a})$. It is based on the fact that

$$
\mathfrak{b}(L)=\mathfrak{b}^{\prime}(L) \otimes \mathfrak{L}^{\otimes 2}
$$

which follows from the definitions. We define $\mathcal{R}_{i, j}$ for $i \in[0,2 \rho+1]$ and $\mathcal{P}_{i, j}$ for $i \in[0,2 \rho]$ as in 2.17 , but replacing $L^{J},{ }^{\prime} L^{J}, r, \delta$ by $\mathfrak{b}(L), \mathfrak{b}^{\prime}(L), 3,2 \rho$. In particular, we have

$$
\mathcal{P}_{i, j}=\mathcal{X}_{4 \rho-i}(i-2 \rho) \otimes\left(\mathfrak{b}^{\prime}(L)\right)^{-4 \rho+i+j} \text { for } i \in[0,2 \rho]
$$

where $\mathcal{X}_{4 \rho-i}$ is a free abelian group of rank $\left(\begin{array}{c}2 \rho \\ i\end{array}\right)$ and $\mathcal{X}_{4 \rho}=\mathbf{Z}$. We have for any $j$ an exact sequence analogous to $2.17(\mathrm{a})$ :

(e) $\quad \cdots \rightarrow \mathcal{P}_{i, j-1} \rightarrow \mathcal{R}_{i+1, j} \rightarrow \mathcal{R}_{i, j} \rightarrow \mathcal{P}_{i, j} \rightarrow \mathcal{R}_{i+1, j+1} \rightarrow \mathcal{R}_{i, j+1} \rightarrow \ldots$,

and we have

$$
\mathcal{R}_{0, j}=(\mathfrak{b}(L))^{j}, \quad \mathcal{P}_{0, j}=\left(\mathfrak{b}^{\prime}(L)\right)^{j-4 \rho}(-2 \rho)
$$

We show:

(f) If $i \in[0,2 \rho+1]$ then $\mathcal{R}_{i, j} \in \mathcal{M} \preceq \tilde{\mathcal{B}}^{2}$.

(g) If $i \in[0,2 \rho+1], j>6 \rho-i+\nu+2 a$ then $\mathcal{R}_{i, j} \in \mathcal{M}^{\prec} \tilde{\mathcal{B}}^{2}$. 
We prove (f), (g) by descending induction on $i$ as in 2.21. If $i=2 \rho+1$ then, since $\mathcal{R}_{2 \rho+1, j}=0$, there is nothing to prove. Now assume that $i \in$ $[0,2 \rho]$. Assume that $\mathbf{L}_{\lambda}^{\dot{w}}$ is a composition factor of $\mathcal{R}_{i, j}$ (without the mixed structure). We must show that $w \cdot \lambda \preceq \mathbf{c}$ and that, if $j>6 \rho-i+\nu+2 a$ then $w \cdot \lambda \prec \mathbf{c}$. Using (e), we see that $\mathbf{L}_{\lambda}^{\dot{w}}$ is a composition factor of $\mathcal{R}_{i+1, j}$ or of $\mathcal{P}_{i, j}$. In the first case, using the induction hypothesis we see that $w \cdot \lambda \preceq \mathbf{c}$ and that, if $j>6 \rho-i+\nu+2 a$ (so that $j>6 \rho-i-1+\nu+2 a$ ), then $w \cdot \lambda \prec \mathbf{c}$. In the second case, $\mathbf{L}_{\lambda}^{\dot{w}}$ is a composition factor of $\left(\mathfrak{b}^{\prime}(L)\right)^{-4 \rho+i+j}$. Using (a), (c), we see that $w \cdot \lambda \preceq \mathbf{c}$ and that, if $j>6 \rho-i+\nu+2 a$ (so that $-4 \rho+i+j>\nu+2 \rho+2 a)$, then $w \cdot \lambda \prec \mathbf{c}$. This proves (f), (g).

We show:

(h) Assume that $i \in[0,2 \rho+1]$. Then $\mathcal{R}_{i, j}$ is mixed of weight $\leq j-i$.

We argue as in 2.22 by descending induction on $i$. If $i=2 \rho+1$ there is nothing to prove. Assume now that $i \leq 2 \rho$. By Deligne's theorem, $\mathfrak{b}^{\prime}(L)$ is mixed of weight $\leq 0$; hence $\left(\mathfrak{b}^{\prime}(L)\right)^{-4 \rho+i+j}$ is mixed of weight $\leq-4 \rho+i+j$ and $\mathcal{X}_{4 \rho-i}(i-2 \rho) \otimes\left(\mathfrak{b}^{\prime}(L)\right)^{-4 \rho+i+j}$ is mixed of weight $\leq-4 \rho+i+j-2(i-2 \rho)=$ $j-i$. In other words, $\mathcal{P}_{i, j}$ is mixed of weight $\leq j-i$. Thus in the exact sequence $\mathcal{R}_{i+1, j} \rightarrow \mathcal{R}_{i, j} \rightarrow \mathcal{P}_{i, j}$ coming from (e) in which $\mathcal{R}_{i+1, j}$ is mixed of weight $\leq j-i-1<j-i$ (by the induction hypothesis) and $\mathcal{P}_{i, j}$ is mixed of weight $\leq j-i$ we must have that $\mathcal{R}_{i, j}$ is mixed of weight $\leq j-i$. This proves $(\mathrm{h})$.

We now prove (d). From (e) we deduce an exact sequence

$$
g r_{j}\left(\mathcal{R}_{1, j}\right) \rightarrow g r_{j}\left(\mathcal{R}_{0, j}\right) \rightarrow g r_{j}\left(\mathcal{P}_{0, j}\right) \rightarrow g r_{j}\left(\mathcal{R}_{1, j+1}\right)
$$

By $(\mathrm{h})$ we have $g r_{j}\left(\mathcal{R}_{1, j}\right)=0$. We have $g r_{j}\left(\mathcal{R}_{0, j}\right)=g r_{j}\left(\mathfrak{b}(L)^{j}\right), g r_{j}\left(\mathcal{P}_{0, j}\right)=$ $g r_{j}\left(\left(\mathfrak{b}^{\prime}(L)\right)^{-4 \rho+j}(-2 \rho)\right)$. Moreover, by (g) we have $\mathcal{R}_{1, j+1} \in \mathcal{D}^{\prec} \tilde{\mathcal{B}}^{2}$ since $j+1>6 \rho-1+\nu+2 a$. It follows that $g r_{j}\left(\mathcal{R}_{1, j+1}\right) \in \mathcal{D}^{\prec} \tilde{\mathcal{B}}^{2}$. Thus the exact sequence above induces an isomorphism as in $(d)$.

We show:

(i) Let $L \in \mathcal{D}(Z)$. Let $L^{\prime} \in \mathcal{M}\left(\tilde{\mathcal{B}}^{2}\right)$ be $G$-equivariant. We have canonically

$$
\mathfrak{b}^{\prime}(L) \circ L^{\prime}=L^{\prime} \circ \mathfrak{b}^{\prime}(L)
$$


Let $R=\mathbf{T} \backslash\left\{\left(\left(x_{0} \mathbf{U}, x_{1} \mathbf{U}, x_{2} \mathbf{U}, x_{3} \mathbf{U}\right), g\right) \in \tilde{\mathcal{B}}^{4} \times G ; g \in x_{2} \mathbf{U} x_{1}^{-1}\right\}$ where $\mathbf{T}$ acts freely by

$$
t:\left(\left(x_{0} \mathbf{U}, x_{1} \mathbf{U}, x_{2} \mathbf{U}, x_{3} \mathbf{U}\right), g\right) \mapsto\left(\left(x_{0} \mathbf{U}, x_{1} t \mathbf{U}, x_{2} t \mathbf{U}, x_{3} \mathbf{U}\right), g\right)
$$

Define $c_{0}: R \rightarrow Z$ by $\left(\left(x_{0} \mathbf{U}, x_{1} \mathbf{U}, x_{2} \mathbf{U}, x_{3} \mathbf{U}\right), g\right) \mapsto \epsilon\left(x_{1} \mathbf{U}, x_{2} \mathbf{U}\right)$.

Define $c_{1}: R \rightarrow \tilde{\mathcal{B}}^{2}$ by $\left(\left(x_{0} \mathbf{U}, x_{1} \mathbf{U}, x_{2} \mathbf{U}, x_{3} \mathbf{U}\right), g\right) \mapsto\left(x_{0} \mathbf{U}, g x_{3} \mathbf{U}\right)$.

Define $c_{2}: R \rightarrow \tilde{\mathcal{B}}^{2}$ by $\left(\left(x_{0} \mathbf{U}, x_{1} \mathbf{U}, x_{2} \mathbf{U}, x_{3} \mathbf{U}\right), g\right) \mapsto\left(g^{-1} x_{0} \mathbf{U}, x_{3} \mathbf{U}\right)$.

Define $c_{3}: R \rightarrow \tilde{\mathcal{B}}^{2}$ by $\left(\left(x_{0} \mathbf{U}, x_{1} \mathbf{U}, x_{2} \mathbf{U}, x_{3} \mathbf{U}\right), g\right) \mapsto\left(x_{0} \mathbf{U}, x_{3} \mathbf{U}\right)$. We have

$$
L^{\prime} \circ \mathfrak{b}^{\prime}(L)=c_{3 !}\left(c_{1}^{*} L^{\prime} \otimes c_{0}^{*} L\right), \mathfrak{b}^{\prime}(L) \circ L^{\prime}=c_{3 !}\left(c_{2}^{*} L^{\prime} \otimes c_{0}^{*} L\right) .
$$

It is enough to show that $c_{1}^{*} L^{\prime}=c_{2}^{*} L^{\prime}$. This follows from the $G$-equivariance of $L^{\prime}$.

(j) If $L \in \mathcal{C}_{0}^{\mathbf{c}} Z, L^{\prime} \in \mathcal{C}^{\mathbf{c}} \tilde{\mathcal{B}}^{2}$, then we have canonically $\underline{\mathfrak{b}}(L) \underline{\circ} L^{\prime}=L^{\prime} \underline{\mathfrak{b}}(L)$.

By $(\mathrm{d})$, it is enough to prove that $\underline{\mathfrak{b}}^{\prime}(L) \underline{\circ} L^{\prime}=L^{\prime} \underline{\mathfrak{b}}^{\prime}(L)$. Using (i) together with (a), (b), (c) and results in 2.23, we see that both sides are equal to

$$
\begin{aligned}
& \frac{g r_{\rho+\nu+3 a}\left(c_{3 !}\left(c_{1}^{*} L^{\prime} \otimes c_{0}^{*} L\right)\right)^{\rho+\nu+3 a}((\rho+\nu+3 a) / 2)}{\quad \underline{g r_{\rho+\nu+3 a}\left(c_{3 !}\left(c_{2}^{*} L^{\prime} \otimes c_{0}^{*} L\right)\right)^{\rho+\nu+3 a}}((\rho+\nu+3 a) / 2) .} .
\end{aligned}
$$

3.15. Let

$$
\left.\mathfrak{Z}=\left\{\left(z_{0} \mathbf{U}, z_{1} \mathbf{U}, z_{2} \mathbf{U}, z_{3} \mathbf{U}\right), g\right) \in \tilde{\mathcal{B}}^{4} \times G ; g \in z_{2} \mathbf{B} z_{1}^{-1}\right\} .
$$

Define $\tilde{\vartheta}: \mathfrak{Z} \rightarrow \tilde{\mathcal{B}}^{4}$ by $\left(\left(z_{0} \mathbf{U}, z_{1} \mathbf{U}, z_{2} \mathbf{U}, z_{3} \mathbf{U}\right), g\right) \mapsto\left(z_{0} \mathbf{U}, z_{1} \mathbf{U}, z_{2} \mathbf{U}, z_{3} \mathbf{U}\right)$. Let

$$
\begin{aligned}
& ' \mathcal{Y}=\left\{\left(\left(x_{0} \mathbf{U}, x_{1} \mathbf{U}, x_{2} \mathbf{U}, x_{3} \mathbf{U}, x_{4} \mathbf{U}\right), g\right) \in \tilde{\mathcal{B}}^{5} \times G ; g \in x_{3} \mathbf{U} x_{0}^{-1}, g \in x_{2} \mathbf{B} x_{1}^{-1}\right\}, \\
& { }^{\prime \prime} \mathcal{Y}=\left\{\left(\left(x_{0} \mathbf{U}, x_{1} \mathbf{U}, x_{2} \mathbf{U}, x_{3} \mathbf{U}, x_{4} \mathbf{U}\right), g\right) \in \tilde{\mathcal{B}}^{5} \times G ; g \in x_{4} \mathbf{U} x_{1}^{-1}, g \in x_{3} \mathbf{B} x_{2}^{-1}\right\},
\end{aligned}
$$

Define $^{\prime} \vartheta:{ }^{\prime} \mathcal{Y} \rightarrow \tilde{\mathcal{B}}^{5},{ }^{\prime \prime} \vartheta:{ }^{\prime \prime} \mathcal{Y} \rightarrow \tilde{\mathcal{B}}^{5}$ by

$$
\left(\left(x_{0} \mathbf{U}, x_{1} \mathbf{U}, x_{2} \mathbf{U}, x_{3} \mathbf{U}, x_{4} \mathbf{U}\right), g\right) \mapsto\left(x_{0} \mathbf{U}, x_{1} \mathbf{U}, x_{2} \mathbf{U}, x_{3} \mathbf{U}, x_{4} \mathbf{U}\right) .
$$


We have isomorphisms ${ }^{\prime} \mathfrak{c}:{ }^{\prime} \mathcal{Y} \stackrel{\sim}{\rightarrow} \mathfrak{Z},{ }^{\prime \prime} \mathfrak{c}:{ }^{\prime \prime} \mathcal{Y} \stackrel{\sim}{\rightarrow} \mathfrak{Z}$ given by

$$
\begin{aligned}
{ }^{\prime} \mathfrak{c}: & \left(\left(x_{0} \mathbf{U}, x_{1} \mathbf{U}, x_{2} \mathbf{U}, x_{3} \mathbf{U}, x_{4} \mathbf{U}\right), g\right) \mapsto\left(\left(x_{0} \mathbf{U}, x_{1} \mathbf{U}, x_{2} \mathbf{U}, x_{4} \mathbf{U}\right), g\right), \\
{ }^{\prime} \mathfrak{c}: & \left(\left(x_{0} \mathbf{U}, x_{1} \mathbf{U}, x_{2} \mathbf{U}, x_{3} \mathbf{U}, x_{4} \mathbf{U}\right), g\right) \mapsto\left(\left(x_{0} \mathbf{U}, x_{2} \mathbf{U}, x_{3} \mathbf{U}, x_{4} \mathbf{U}\right), g\right) .
\end{aligned}
$$

Define $^{\prime} d: \tilde{\mathcal{B}}^{5} \rightarrow \tilde{\mathcal{B}}^{4},{ }^{\prime \prime} d: \tilde{\mathcal{B}}^{5} \rightarrow \tilde{\mathcal{B}}^{4}$ by

$$
\begin{aligned}
{ }^{\prime} d: & \left(x_{0} \mathbf{U}, x_{1} \mathbf{U}, x_{2} \mathbf{U}, x_{3} \mathbf{U}, x_{4} \mathbf{U}\right) \mapsto\left(x_{0} \mathbf{U}, x_{1} \mathbf{U}, x_{2} \mathbf{U}, x_{4} \mathbf{U}\right), \\
{ }^{\prime \prime} d: & \left(x_{0} \mathbf{U}, x_{1} \mathbf{U}, x_{2} \mathbf{U}, x_{3} \mathbf{U}, x_{4} \mathbf{U}\right) \mapsto\left(x_{0} \mathbf{U}, x_{2} \mathbf{U}, x_{3} \mathbf{U}, x_{4} \mathbf{U}\right) .
\end{aligned}
$$

We fix $w, u$ in $W$ and $\lambda, \lambda^{\prime}$ in $\mathfrak{s}$ such that $w(\lambda)=\lambda^{\prime}$. The smooth subvarieties

$$
\begin{aligned}
\mathfrak{U} & =\left\{\left(\left(x_{0} \mathbf{U}, x_{1} \mathbf{U}, x_{2} \mathbf{U}, x_{3} \mathbf{U}, x_{4} \mathbf{U}\right), g\right) \in{ }^{\prime} \mathcal{Y} ; x_{1}^{-1} x_{2} \in G_{w}, x_{3}^{-1} x_{4} \in G_{u}\right\}, \\
\mathcal{U} & =\left\{\left(\left(x_{0} \mathbf{U}, x_{1} \mathbf{U}, x_{2} \mathbf{U}, x_{3} \mathbf{U}\right), g\right) \in \mathfrak{Z} ; x_{1}^{-1} x_{2} \in G_{w}, x_{0}^{-1} g^{-1} x_{3} \in G_{u}\right\}, \\
{ }^{\prime} \mathcal{U} & =\left\{\left(\left(x_{0} \mathbf{U}, x_{1} \mathbf{U}, x_{2} \mathbf{U}, x_{3} \mathbf{U}, x_{4} \mathbf{U}\right), g\right) \in{ }^{\prime \prime} \mathcal{Y} ; x_{2}^{-1} x_{3} \in G_{w}, x_{0}^{-1} x_{1} \in G_{u}\right\},
\end{aligned}
$$

of ${ }^{\prime} \mathcal{Y}, \mathfrak{Z},{ }^{\prime \prime} \mathcal{Y}$ correspond to each other under the isomorphisms ${ }^{\prime} \mathcal{Y} \stackrel{{ }^{\mathfrak{c}}}{\rightarrow} \mathfrak{Z} \leftarrow$ Moreover, the maps ${ }^{\prime} \sigma:{ }^{\prime} \mathcal{U} \rightarrow Z, \sigma: \mathcal{U} \rightarrow Z,{ }^{\prime \prime} \sigma:{ }^{\prime \prime} \mathcal{U} \rightarrow Z$ given by

$$
\begin{aligned}
\left(\left(x_{0} \mathbf{U}, x_{1} \mathbf{U}, x_{2} \mathbf{U}, x_{3} \mathbf{U}, x_{4} \mathbf{U}\right), g\right) & \mapsto \epsilon\left(x_{1} \mathbf{U}, x_{2} \mathbf{U}\right), \\
\left(\left(x_{0} \mathbf{U}, x_{1} \mathbf{U}, x_{2} \mathbf{U}, x_{3} \mathbf{U}\right), g\right) & \mapsto \epsilon\left(x_{1} \mathbf{U}, x_{2} \mathbf{U}\right), \\
\left(\left(x_{0} \mathbf{U}, x_{1} \mathbf{U}, x_{2} \mathbf{U}, x_{3} \mathbf{U}, x_{4} \mathbf{U}\right), g\right) & \mapsto \epsilon\left(x_{2} \mathbf{U}, x_{3} \mathbf{U}\right),
\end{aligned}
$$

correspond to each other under the isomorphisms ${ }^{\prime} \mathcal{Y} \stackrel{\prime \mathfrak{c}}{\rightarrow} \mathfrak{Z} \stackrel{\prime \prime}{\leftarrow}{ }^{\prime \prime} \mathcal{Y}$.

Also the maps ${ }^{\prime} \tilde{\sigma}:{ }^{\prime} \mathcal{U} \rightarrow \tilde{\mathcal{B}}^{2}, \tilde{\sigma}: \mathcal{U} \rightarrow \tilde{\mathcal{B}}^{2}$, given by

$$
\begin{aligned}
\left(\left(x_{0} \mathbf{U}, x_{1} \mathbf{U}, x_{2} \mathbf{U}, x_{3} \mathbf{U}, x_{4} \mathbf{U}\right), g\right) & \mapsto\left(x_{3} \mathbf{U}, x_{4} \mathbf{U}\right), \\
\left(\left(x_{0} \mathbf{U}, x_{1} \mathbf{U}, x_{2} \mathbf{U}, x_{3} \mathbf{U}\right), g\right) & \mapsto\left(g x_{0} \mathbf{U}, x_{3} \mathbf{U}\right)
\end{aligned}
$$

correspond to each other under the isomorphism ${ }^{\prime} \mathcal{Y} \stackrel{\text { cc }}{\rightarrow} \mathfrak{Z}$ and the maps $\tilde{\sigma}_{1}$ : $\mathcal{U} \rightarrow \tilde{\mathcal{B}}^{2},{ }^{\prime \prime} \tilde{\sigma}:{ }^{\prime \prime} \mathcal{U} \rightarrow \tilde{\mathcal{B}}^{2}$ given by

$$
\begin{aligned}
\left(\left(x_{0} \mathbf{U}, x_{1} \mathbf{U}, x_{2} \mathbf{U}, x_{3} \mathbf{U}\right), g\right) & \mapsto\left(x_{0} \mathbf{U}, g^{-1} x_{3} \mathbf{U}\right), \\
\left(\left(x_{0} \mathbf{U}, x_{1} \mathbf{U}, x_{2} \mathbf{U}, x_{3} \mathbf{U}, x_{4} \mathbf{U}\right), g\right) & \mapsto\left(x_{0} \mathbf{U}, x_{1} \mathbf{U}\right),
\end{aligned}
$$

correspond to each other under the isomorphism $\mathfrak{Z} \leftarrow$ ' the local systems ${ }^{\prime} \sigma^{*} \mathcal{L}_{\lambda}^{\dot{w}}, \sigma^{*} \mathcal{L}_{\lambda}^{\dot{w}},{ }^{\prime \prime} \sigma^{*} \mathcal{L}_{\lambda}^{\dot{w}}$ correspond to each other under the 
isomorphisms ' $\mathcal{Y} \stackrel{\text { 'c }}{\rightarrow} \mathfrak{Z} \stackrel{\text { "Ic }}{\leftarrow}$ "Y $\mathcal{Y}$; the local systems ${ }^{\prime} \tilde{\sigma}^{*} L_{\lambda^{\prime}}^{\dot{u}}, \tilde{\sigma}^{*} L_{\lambda^{\prime}}^{\dot{u}}$ correspond to each other under the isomorphism ' $\mathcal{Y} \stackrel{\text { 'c }}{\rightarrow} \mathfrak{Z}$; the local systems $\tilde{\sigma}_{1}^{*} L_{\lambda^{\prime}}^{\dot{u}},{ }^{\prime \prime} \tilde{\sigma}^{*} L_{\lambda^{\prime}}^{\dot{u}}$ correspond to each other under the isomorphism $\mathfrak{Z} \longleftarrow \mathfrak{c} \subset \mathcal{}$. Moreover, we have $\tilde{\sigma}^{*} L_{\lambda^{\prime}}^{\dot{u}}=\tilde{\sigma}_{1}^{*} L_{\lambda^{\prime}}^{\dot{u}}$ by the $G$-equivariance of $L_{\lambda^{\prime}}^{\dot{u}}$. Let ${ }^{\prime} K, K,{ }^{\prime \prime} K$ be the intersection cohomology complex of the closure of $\mathcal{U}, \mathcal{U},{ }^{\prime \prime} \mathcal{U}$ respectively with coefficients in the local system

$$
{ }^{\prime} \sigma^{*} \mathcal{L}_{\lambda}^{\dot{w}} \otimes{ }^{\prime} \tilde{\sigma}^{*} L_{\lambda^{\prime}}^{\dot{u}}, \sigma^{*} \mathcal{L}_{\lambda}^{\dot{w}} \otimes \tilde{\sigma}^{*} L_{\lambda^{\prime}}^{\dot{u}},{ }^{\prime \prime} \sigma^{*} \mathcal{L}_{\lambda}^{\dot{w}} \otimes " \tilde{\sigma}^{*} L_{\lambda^{\prime}}^{\dot{u}},
$$

on ' $\mathcal{U}, \mathcal{U}$, " $\mathcal{U}$ (respectively) extended by 0 on the complement of this closure in ' $\mathcal{Y}, \mathfrak{Z},{ }^{\prime \prime} \mathcal{Y}$. We see that ${ }^{\prime} K, K,{ }^{\prime \prime} K$ correspond to each other under the isomorphisms $\mathcal{Y}^{\prime} \stackrel{\mathfrak{c}}{\rightarrow} \mathfrak{Z} \stackrel{\text { "cㅢ }}{\leftarrow} \mathcal{Y}$. Hence we have ${ }^{\prime} \mathfrak{c} !\left({ }^{\prime} K\right)=K={ }^{\prime \prime} \mathfrak{c} !\left({ }^{\prime \prime} K\right)$. Using this and the commutative diagram

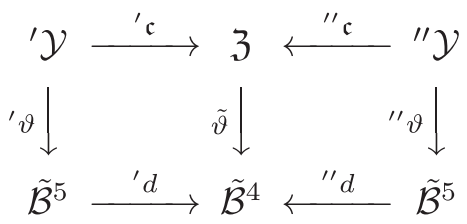

we see that

$$
\left.{ }^{\prime} d ! \vartheta_{!}^{\prime} \vartheta_{!}^{\prime} K\right)={ }^{\prime \prime} d_{!}^{\prime \prime} \vartheta_{!}\left({ }^{\prime \prime} K\right)
$$

(Both sides are equal to $\tilde{\vartheta}_{!} K$.)

3.16. In this subsection we study the functor ' ${ }^{\prime} d_{!}: \mathcal{D}_{m}\left(\tilde{\mathcal{B}}^{5}\right) \rightarrow \mathcal{D}_{m}\left(\tilde{\mathcal{B}}^{4}\right)$. Let $\mathbf{w}=\left(w_{1}, w_{2}, w_{3}, w_{4}\right), \boldsymbol{\lambda}=\left(\lambda_{1}, \lambda_{2}, \lambda_{3}, \lambda_{4}\right), \boldsymbol{\omega}=\left(\omega_{1}, \omega_{2}, \omega_{3}, \omega_{4}\right)$ (with $\omega_{i} \in$ $\left.\kappa_{q}^{-1}\left(w_{i}\right)\right)$. Assume that $w_{4} \cdot \lambda_{4} \preceq$ c. Let $K=M_{\lambda}^{\omega,[1,4]}\langle|\mathbf{w}|+5 \rho+\nu\rangle \in \mathcal{D}_{m}\left(\tilde{\mathcal{B}}^{5}\right)$. We show:

(a) If $h>a+\rho$ then $\left({ }^{\prime} d_{!} K\right)^{h} \in{ }^{\prime} \mathcal{M}^{\prec}\left(\tilde{\mathcal{B}}^{4}\right)$. Moreover,

$$
\begin{aligned}
& \underline{g r_{a+\rho}\left(\left({ }^{\prime} d K\right)^{a+\rho}\right)}((a+\rho) / 2)=\oplus_{y^{\prime} \in W ; y^{\prime-1} \cdot \lambda_{4} \in \mathbf{c}} \operatorname{Hom}_{\mathcal{C}^{c} \tilde{\mathcal{B}}^{2}}\left(\mathbf{L}_{\lambda_{4}}^{\dot{y}^{\prime-1}}, \mathbf{L}_{\lambda_{3}}^{\omega_{3}} \mathbf{L}_{\lambda_{4}}^{\omega_{4}}\right) \\
& \otimes M_{\lambda_{1}, \lambda_{2}, \lambda_{4}}^{\omega_{1}, \omega_{2}, \dot{y}^{\prime}-1,[1,3]}\left\langle\left|w_{1}\right|+\left|w_{2}\right|+\left|y^{\prime}\right|+4 \rho+\nu\right\rangle \text {. }
\end{aligned}
$$

We shall apply 24, 1.12] with $\Phi: \mathcal{D}_{m}\left(Y_{1}\right) \rightarrow \mathcal{D}_{m}\left(Y_{2}\right)$ replaced by $\Phi_{0}:$ $\mathcal{D}_{m}\left(\tilde{\mathcal{B}}^{2}\right) \rightarrow \mathcal{D}_{m}\left(\tilde{\mathcal{B}}^{4}\right), M \mapsto p_{01}^{*} L_{\lambda_{1}}^{\omega_{\sharp} \sharp}\left\langle\left|w_{1}\right|\right\rangle \otimes p_{12}^{*} L_{\lambda_{2}}^{\omega_{2} \sharp}\left\langle\left|w_{2}\right|\right\rangle \otimes p_{23}^{*} M\langle\rho-\nu\rangle$ and with $\mathcal{D} \preceq\left(Y_{1}\right), \mathcal{D} \preceq\left(Y_{2}\right)$ replaced by ${ }_{4} \mathcal{D} \preceq\left(\tilde{\mathcal{B}}^{2}\right),{ }_{4} \mathcal{D} \preceq\left(\tilde{\mathcal{B}}^{4}\right)$, see 3.15 . We shall take $\mathbf{X}$ in loc.cit. equal to $\Xi:=L_{\lambda_{3}}^{\omega_{3} \sharp} \circ L_{\lambda_{4}}^{\omega_{4} \sharp}\left\langle\left|w_{3}\right|+\left|w_{4}\right|+4 \rho+2 \nu\right\rangle$. The conditions 
of loc.cit. are satisfied: those concerning $\mathbf{X}$ are satisfied with $c^{\prime}=a-\nu$ (see 2.23); those concerning $\Phi_{0}$ are satisfied with $c=\rho+\nu$ (using the definitions). Since ' $d_{!} K=\Phi_{0}(\Xi)$ and $c+c^{\prime}=a+\rho$ we see that the first sentence in (a) holds; moreover, we see that, setting $K_{1}=g r_{a+\rho}\left(\left({ }^{\prime} d_{!} K\right)^{a+\rho}\right)((a+\rho) / 2)$, we have

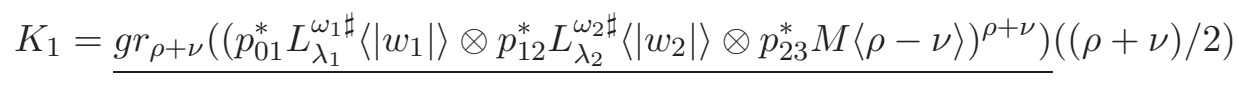

where

$$
M=\mathbf{L}_{\lambda_{3}}^{\omega_{3}} \mathbf{L}_{\lambda_{4}}^{\omega_{4}}=\oplus_{y^{\prime-1} \cdot \eta \in \mathbf{c}} \operatorname{Hom}_{\mathcal{C}^{\mathbf{c}} \tilde{\mathcal{B}}^{2}}\left(\mathbf{L}_{\eta}^{\dot{y}^{\prime-1}}, \mathbf{L}_{\lambda_{3}}^{\omega_{3}} \mathbf{L}_{\lambda_{4}}^{\omega_{4}}\right) \mathbf{L}_{\eta}^{\dot{y}^{\prime-1}}
$$

From $2.13(\mathrm{c})$ we see that $\eta$ above must satisfy $\eta=\lambda_{4}$. Thus we have

$$
\begin{aligned}
K_{1}= & \oplus_{y^{\prime} \in W ; y^{\prime-1} \cdot \lambda_{4} \in \mathbf{c}} \operatorname{Hom}_{\mathcal{C}^{\mathbf{c}} \tilde{\mathcal{B}}^{2}}\left(\mathbf{L}_{\lambda_{4}}^{\dot{y}^{\prime-1}}, \mathbf{L}_{\lambda_{3}}^{\omega_{3}} \mathbf{L}_{\lambda_{4}}^{\omega_{4}}\right) \\
& \stackrel{\left.\otimes r_{\rho+\nu}\left(M_{\lambda_{1}, \lambda_{2}, \lambda_{4}}^{\omega_{1}, \omega_{2}, \dot{y}^{\prime-1},[1,3]}\left\langle\left|w_{1}\right|+\left|w_{2}\right|+\left|y^{\prime}\right|+3 \rho\right\rangle\right)^{\rho+\nu}\right)}{ }((\rho+\nu) / 2) .
\end{aligned}
$$

It remains to use that

$$
\begin{aligned}
& \frac{\left.g r_{\rho+\nu}\left(M_{\lambda_{1}, \lambda_{2}, \lambda_{4}}^{\omega_{1}, \omega_{2}, \dot{y}^{\prime-1},[1,3]}\left\langle\left|w_{1}\right|+\left|w_{2}\right|+\left|y^{\prime}\right|+3 \rho\right\rangle\right)^{\rho+\nu}\right)}{\quad=}((\rho+\nu) / 2) \\
& \quad=\frac{\left.g r_{\rho+\nu}\left(M_{\lambda_{1}, \lambda_{2}, \lambda_{4}}^{\omega_{1}, \omega_{2}, \dot{y}^{\prime-1},[1,3]}\left\langle\left|w_{1}\right|+\left|w_{2}\right|+\left|y^{\prime}\right|+4 \rho+\nu\right\rangle\right)^{0}(-(r+\nu) / 2)\right)}{M_{\lambda_{1}, \lambda_{2}, \lambda_{4}}^{\omega_{1}, \omega_{2}, \dot{y}^{\prime}-1},[1,3]}\left\langle\left|w_{1}\right|+\left|w_{2}\right|+\left|y^{\prime}\right|+4 \rho+\nu\right\rangle .
\end{aligned}
$$

We state the following properties of the functor ${ }^{\prime} d_{!}: \mathcal{D}_{m}\left(\tilde{\mathcal{B}}^{5}\right) \rightarrow \mathcal{D}_{m}\left(\tilde{\mathcal{B}}^{4}\right)$.

(b) If $K \in{ }_{4} \mathcal{D} \preceq\left(\tilde{\mathcal{B}}^{5}\right)$ then ${ }^{\prime} d_{!}(K) \in{ }_{4} \mathcal{D} \preceq\left(\tilde{\mathcal{B}}^{4}\right)$.

(c) If $K \in{ }_{4} \mathcal{D}^{\prec}\left(\tilde{\mathcal{B}}^{5}\right)$ then ${ }^{\prime} d_{!}(K) \in{ }_{4} \mathcal{D}^{\prec}\left(\tilde{\mathcal{B}}^{4}\right)$.

(d) If $K \in{ }_{4} \mathcal{M} \preceq\left(\tilde{\mathcal{B}}^{5}\right)$ and $h>a+\rho$ then $\left({ }^{\prime} d_{!}(K)\right)^{h} \in{ }_{4} \mathcal{M}^{\prec}\left(\tilde{\mathcal{B}}^{4}\right)$.

We prove (b). We can assume that $K$ is as in the first paragraph of this subsection. It is enough to show that for $j \in \mathbf{Z}$ we have $\left(\Phi_{0}(\Xi)\right)^{j} \in{ }_{4} \mathcal{M} \preceq\left(\tilde{\mathcal{B}}^{4}\right)$ (with $\Phi_{0}, \Xi$ as above). It is enough to show that $\Phi_{0}\left(\Xi^{j^{\prime}}\right) \in{ }_{4} \mathcal{M} \preceq\left(\tilde{\mathcal{B}}^{4}\right)$ for any $j^{\prime} \in \mathbf{Z}$. This follows from the fact that $\Xi^{j^{\prime}} \in{ }_{4} \mathcal{M} \preceq\left(\tilde{\mathcal{B}}^{2}\right)$ (see $\left.2.23(\mathrm{~b})\right)$ and the fact that $\Phi_{0}$ carries ${ }_{4} \mathcal{D} \preceq\left(\tilde{\mathcal{B}}^{2}\right)$ to ${ }_{4} \mathcal{D} \preceq\left(\tilde{\mathcal{B}}^{4}\right)$. Thus (b) holds. A similar proof gives (c). We prove (d). We can assume that $K$ is as in (a). Then the result follows from (a). 
3.17. In this subsection we study the functor ${ }^{\prime \prime} d_{!}: \mathcal{D}_{m}\left(\tilde{\mathcal{B}}^{5}\right) \rightarrow \mathcal{D}_{m}\left(\tilde{\mathcal{B}}^{4}\right)$. Let $\mathbf{w}=\left(w_{1}, w_{2}, w_{3}, w_{4}\right), \boldsymbol{\lambda}=\left(\lambda_{1}, \lambda_{2}, \lambda_{3}, \lambda_{4}\right), \boldsymbol{\omega}=\left(\omega_{1}, \omega_{2}, \omega_{3}, \omega_{4}\right)\left(\right.$ with $\omega_{i} \in$ $\left.\kappa_{q}^{-1}\left(w_{i}\right)\right)$. Assume that $w_{1} \cdot \lambda_{1} \preceq \mathbf{c}$. Let $K=M_{\boldsymbol{\lambda}}^{\boldsymbol{\omega},[1,4]}\langle|\mathbf{w}|+5 \rho+\nu\rangle \in \mathcal{D}_{m}\left(\tilde{\mathcal{B}}^{5}\right)$. We show:

(a) If $h>a+\rho$ then $\left({ }^{\prime \prime} d_{!} K\right)^{h} \in{ }^{\prime} \mathcal{M}^{\prec}\left(\tilde{\mathcal{B}}^{4}\right)$. Moreover,

$$
\begin{aligned}
\frac{g r_{a+\rho}\left(\left({ }^{\prime \prime} d_{!} K\right)^{a+\rho}\right)}{((a+\rho) / 2)=} & \bigoplus_{y^{\prime} \in W ; y^{\prime} \cdot \lambda_{2} \in \mathbf{c}} \operatorname{Hom}_{\mathcal{C}^{\mathbf{c}} \tilde{\mathcal{B}}^{2}}\left(\mathbf{L}_{\lambda_{2}}^{\dot{y}^{\prime}}, \mathbf{L}_{\lambda_{1}}^{\omega_{1}} \underline{\mathbf{L}}_{\lambda_{2}}^{\omega_{2}}\right) \\
& \otimes M_{\lambda_{2}, \lambda_{3}, \lambda_{4}}^{\dot{y}^{\prime}, \omega_{3}, \omega_{4},[1,3]}\left\langle\left|w_{3}\right|+\left|w_{4}\right|+\left|y^{\prime}\right|+4 \rho+\nu\right\rangle
\end{aligned}
$$

We shall apply [24, 1.12] with $\Phi: \mathcal{D}_{m}\left(Y_{1}\right) \rightarrow \mathcal{D}_{m}\left(Y_{2}\right)$ replaced by $\Phi_{0}:$ $\mathcal{D}_{m}\left(\tilde{\mathcal{B}}^{2}\right) \rightarrow \mathcal{D}_{m}\left(\tilde{\mathcal{B}}^{4}\right), M \mapsto p_{01}^{*} M \otimes p_{12}^{*} L_{\lambda_{3}}^{\omega_{3} \sharp}\left\langle\left|w_{3}\right|\right\rangle \otimes p_{23}^{*} L_{\lambda_{4}}^{\omega_{4} \sharp}\left\langle\left|w_{4}\right|\right\rangle\langle\rho-\nu\rangle$ and with $\mathcal{D} \preceq\left(Y_{1}\right), \mathcal{D} \preceq\left(Y_{2}\right)$ replaced by ${ }_{1} \mathcal{D} \preceq\left(\tilde{\mathcal{B}}^{2}\right),{ }_{1} \mathcal{D} \preceq\left(\tilde{\mathcal{B}}^{4}\right)$, see 3.15 . We shall take $\mathbf{X}$ in loc.cit. equal to $\Xi:=L_{\lambda_{1}}^{\omega_{1} \sharp} \circ L_{\lambda_{2}}^{\omega_{2} \sharp}\left\langle\left|w_{1}\right|+\left|w_{2}\right|+4 \rho+2 \nu\right\rangle$. The conditions of loc.cit. are satisfied: those concerning $\mathbf{X}$ are satisfied with $c^{\prime}=a-\nu$ (see 2.23); those concerning $\Phi_{0}$ are satisfied with $c=\rho+\nu$ (using the definitions). Since " $d_{!} K=\Phi_{0}(\Xi)$ and $c+c^{\prime}=a+\rho$ we see that the first sentence in (a) holds; moreover we see that, setting $K_{1}=\underline{g r_{a+\rho}\left(\left({ }^{\prime} d_{!} K\right)^{a+\rho}\right)}((a+\rho) / 2)$, we have

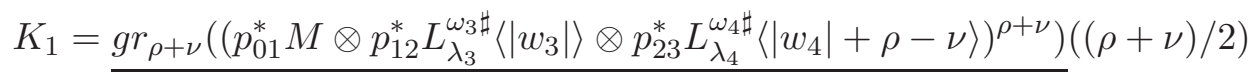

where

$$
M=\mathbf{L}_{\lambda_{1}}^{\omega_{1}} \mathbf{L}_{\lambda_{2}}^{\omega_{2}}=\oplus_{y^{\prime} \cdot \eta \in \mathbf{c}} \operatorname{Hom}_{\mathcal{C}^{c} \tilde{\mathcal{B}}^{2}}\left(\mathbf{L}_{\eta}^{\dot{y}^{\prime}}, \mathbf{L}_{\lambda_{1}}^{\omega_{1}} \mathbf{L}_{\lambda_{2}}^{\omega_{2}}\right) \mathbf{L}_{\eta}^{\dot{y}^{\prime}}
$$

From 2.13(c) we see that $\eta$ must satisfy $\eta=\lambda_{2}$. Thus we have

$$
\begin{aligned}
K_{1}= & \oplus_{y^{\prime} \in W ; y^{\prime} \cdot \lambda_{2} \in \mathbf{c}} \operatorname{Hom}_{\mathcal{C}^{\mathbf{c}} \tilde{\mathcal{B}}^{2}}\left(\mathbf{L}_{\lambda_{2}}^{\dot{y}^{\prime}}, \mathbf{L}_{\lambda_{1}}^{\omega_{1}} \underline{\mathbf{L}_{\lambda_{2}}^{\omega_{2}}}\right) \\
& \underline{\left.\otimes r_{\rho+\nu}\left(M_{\lambda_{2}, \lambda_{3}, \lambda_{4}}^{\dot{y}^{\prime}, \omega_{3}, \omega_{4},[1,3]}\left\langle\left|w_{3}\right|+\left|w_{4}\right|+\left|y^{\prime}\right|+3 \rho\right\rangle\right)^{\rho+\nu}\right)}((\rho+\nu) / 2) .
\end{aligned}
$$

It remains to use that

$$
\begin{aligned}
& \underline{\left.g r_{\rho+\nu}\left(M_{\lambda_{2}, \lambda_{3}, \lambda_{4}}^{\dot{y}^{\prime}, \omega_{3}, \omega_{4},[1,3]}\left\langle\left|w_{3}\right|+\left|w_{4}\right|+\left|y^{\prime}\right|+3 \rho\right\rangle\right)^{\rho+\nu}\right)}((\rho+\nu) / 2) \\
& \left.=g r_{\rho+\nu}\left(M_{\lambda_{2}, \lambda_{3}, \lambda_{4}}^{\dot{y}^{\prime}, \omega_{3}, \omega_{4},[1,3]}\left\langle\left|w_{3}\right|+\left|w_{4}\right|+\left|y^{\prime}\right|+4 \rho+\nu\right\rangle\right)^{0}(-(r+\nu) / 2)\right)((\rho+\nu) / 2) \\
& =M_{\lambda_{2}, \lambda_{3}, \lambda_{4}}^{\dot{y}^{\prime}, \omega_{3}, \omega_{4},[1,3]}\left\langle\left|w_{3}\right|+\left|w_{4}\right|+\left|y^{\prime}\right|+4 \rho+\nu\right\rangle \text {. }
\end{aligned}
$$

We state the following properties of the functor ${ }^{\prime \prime} d_{!}: \mathcal{D}_{m}\left(\tilde{\mathcal{B}}^{5}\right) \rightarrow \mathcal{D}_{m}\left(\tilde{\mathcal{B}}^{4}\right)$. 
(b) If $K \in{ }_{1} \mathcal{D} \preceq\left(\tilde{\mathcal{B}}^{5}\right)$ then ${ }^{\prime \prime} d_{!}(K) \in{ }_{1} \mathcal{D} \preceq\left(\tilde{\mathcal{B}}^{4}\right)$.

(c) If $K \in{ }_{1} \mathcal{D}^{\prec}\left(\tilde{\mathcal{B}}^{5}\right)$ then $" d_{!}(K) \in{ }_{1} \mathcal{D}^{\prec}\left(\tilde{\mathcal{B}}^{4}\right)$.

(d) If $K \in{ }_{1} \mathcal{M} \preceq\left(\tilde{\mathcal{B}}^{5}\right)$ and $h>a+\rho$ then $\left({ }^{\prime \prime} d_{!}(K)\right)^{h} \in{ }_{1} \mathcal{M}^{\prec}\left(\tilde{\mathcal{B}}^{4}\right)$.

The proof of (b), (c), (d) is completely similar to that of 3.16(b), (c), (d).

3.18. Let $w \cdot \lambda \in \widetilde{W} \mathfrak{s}, u \cdot \lambda^{\prime} \in \mathbf{c}$. We shall apply [24, 1.12] with $\Phi$ : $\mathcal{D}_{m}\left(Y_{1}\right) \rightarrow \mathcal{D}_{m}\left(Y_{2}\right)$ replaced by ${ }^{\prime} d !: \mathcal{D}_{m}\left(\tilde{\mathcal{B}}^{5}\right) \rightarrow \mathcal{D}_{m}\left(\tilde{\mathcal{B}}^{4}\right)$ and with $\mathcal{D}^{\preceq}\left(Y_{1}\right)$, $\mathcal{D} \preceq\left(Y_{2}\right)$ replaced by ${ }_{4} \mathcal{D} \preceq\left(\tilde{\mathcal{B}}^{5}\right),{ }_{4} \mathcal{D} \preceq\left(\tilde{\mathcal{B}}^{4}\right)$, see 3.15 . We shall take $\mathbf{X}$ in loc.cit. equal to $\Xi={ }^{\prime} \vartheta_{!}\left({ }^{\prime} K\right)$ as in $3.15,\left(w_{2}, w_{4}\right)=(w, u),\left(\lambda_{2}, \lambda_{4}\right)=\left(\lambda, \lambda^{\prime}\right)$. The conditions of loc.cit. are satisfied: those concerning $\mathbf{X}$ are satisfied with $c^{\prime}=k=|w|+|u|+3 \nu+5 \rho$ (see 3.8(c)); those concerning $\Phi$ are satisfied with $c=a+\rho$ (see 3.16). We see that

$$
\begin{aligned}
& \underline{g r_{a+\rho+k}\left(\left({ }^{\prime} d_{!}^{\prime} \vartheta_{!}\left({ }^{\prime} K\right)\right)^{a+\rho+k}\right)}((a+\rho+k) / 2) \\
& \left.\left.=\underline{g r_{a+\rho}\left(\left({ }^{\prime} d_{!} \underline{g r_{k}}\left(\left({ }^{\prime} \vartheta_{!}\left({ }^{\prime} K\right)\right)^{k}\right)\right.\right.}(k / 2)\right)^{a+\rho}\right)((a+\rho) / 2) .
\end{aligned}
$$

Using 3.11(a) we have:

$$
\begin{aligned}
\left.g r_{k}\left({ }^{\prime} \vartheta_{!}\left({ }^{\prime} K\right)\right)^{k}\right)(k / 2) & =\oplus_{y \in W} M_{\lambda, \lambda, y(\lambda), \lambda^{\prime}}^{\dot{y}, \dot{w}, \dot{y}^{-1}, \dot{u},[1,4]}\langle 2|y|+|w|+|u|+5 \rho+\nu\rangle \\
& =\underline{g r_{k}\left({ }^{\prime} \vartheta_{!}\left({ }^{\prime} K\right)\right)^{k}(k / 2) .}
\end{aligned}
$$

Hence using 3.16(a) we have

$$
\begin{gathered}
\frac{g r_{a+\rho}\left(\left({ }^{\prime} d_{!} g r_{k}\left(\left({ }^{\prime} \vartheta_{!}\left({ }^{\prime} K\right)\right)^{k}\right)(k / 2)\right)^{a+\rho}\right)}{}((a+\rho) / 2) \\
=\oplus_{y \in W} \oplus_{y^{\prime} \in W ; y^{\prime-1} \cdot \lambda^{\prime} \in \mathbf{c}} \operatorname{Hom}_{\mathcal{C}^{\mathbf{c}} \tilde{\mathcal{B}}^{2}}\left(\mathbf{L}_{\lambda^{\prime}}^{\dot{y}^{\prime-1}}, \mathbf{L}_{y(\lambda)}^{\dot{y}^{-1}} \mathbf{L}_{\lambda^{\prime}}^{\dot{u}}\right) \\
\otimes M_{\lambda, \lambda, \lambda^{\prime}}^{\dot{y}, \dot{w}, \dot{y}^{\prime-1},[1,3]}\left\langle|y|+|w|+\left|y^{\prime}\right|+4 \rho+\nu\right\rangle .
\end{gathered}
$$

Since $y^{\prime-1} \cdot \lambda^{\prime} \in \mathbf{c}, u \cdot \lambda^{\prime} \in \mathbf{c}$, for $y \in W$ we have

$$
\operatorname{Hom}_{\mathcal{C}^{\mathbf{c}} \tilde{\mathcal{B}}^{2}}\left(\mathbf{L}_{\lambda^{\prime}}^{\dot{y}^{\prime-1}}, \mathbf{L}_{y(\lambda)}^{\dot{y}^{-1}} \underline{-} \mathbf{L}_{\lambda^{\prime}}^{\dot{u}}\right)=0
$$

unless $y^{-1} \cdot y(\lambda) \in \mathbf{c}($ see 2.26$)$ or equivalently (see $\left.1.9(\mathrm{Q} 10), 1.11\right), y \cdot \lambda \in \mathbf{c}$. Thus we have

$$
\left.\left.\underline{g r_{a+\rho+k}\left(\left({ }^{\prime} d ! \vartheta_{!}^{\prime}\right.\right.}\left({ }^{\prime} K\right)\right)^{a+\rho+k}\right)((a+\rho+k) / 2)
$$




$$
\begin{aligned}
= & \oplus_{y \in W ; y \cdot \lambda \in \mathbf{c}} \oplus_{y^{\prime} \in W ; y^{\prime-1} \cdot \lambda^{\prime} \in \mathbf{c}} \operatorname{Hom}_{\mathcal{C}^{\mathbf{c}} \tilde{\mathcal{B}}^{2}}\left(\mathbf{L}_{\lambda^{\prime}}^{\dot{y}^{\prime-1}}, \mathbf{L}_{y(\lambda)}^{\dot{y}^{-1} \mathrm{o}} \mathbf{L}_{\lambda^{\prime}}^{\dot{u}}\right) \\
& \otimes M_{\lambda, \lambda, \lambda^{\prime}}^{\dot{y}, \dot{w}, \dot{y}^{\prime-1},[1,3]}\left\langle|y|+|w|+\left|y^{\prime}\right|+4 \rho+\nu\right\rangle .
\end{aligned}
$$

The last Hom-space is zero unless $y^{\prime-1}\left(\lambda^{\prime}\right)=\lambda$ hence

$$
\begin{aligned}
& \frac{g r_{a+\rho+k}\left(\left({ }^{\prime} d_{!}^{\prime} \vartheta_{!}\left({ }^{\prime} K\right)\right)^{a+\rho+k}\right)}{=}((a+\rho+k) / 2) \\
& =\oplus_{y \in W ; y \cdot \lambda \in \mathbf{c}} \oplus_{y^{\prime} \in W ; y^{\prime-1} \cdot y^{\prime}(\lambda) \in \mathbf{c}} \operatorname{Hom}_{\mathcal{C}^{\mathbf{c}} \tilde{\mathcal{B}}^{2}}\left(\mathbf{L}_{y^{\prime}(\lambda)}^{\dot{y}^{\prime-1}}, \mathbf{L}_{y(\lambda)}^{\dot{y}^{-1}} \mathbf{L}_{\lambda^{\prime}}^{\dot{u}}\right) \\
& \quad \otimes M_{\lambda, \lambda, \lambda^{\prime}}^{\dot{y}, \dot{w}, \dot{y}^{\prime-1},[1,3]}\left\langle|y|+|w|+\left|y^{\prime}\right|+4 \rho+\nu\right\rangle .
\end{aligned}
$$

3.19. In the setup of 3.18 we shall apply [24, 1.12] with $\Phi: \mathcal{D}_{m}\left(Y_{1}\right) \rightarrow$ $\mathcal{D}_{m}\left(Y_{2}\right)$ replaced by ${ }^{\prime \prime} d_{!}: \mathcal{D}_{m}\left(\tilde{\mathcal{B}}^{5}\right) \rightarrow \mathcal{D}_{m}\left(\tilde{\mathcal{B}}^{4}\right)$ and with $\mathcal{D}^{\preceq}\left(Y_{1}\right), \mathcal{D}^{\preceq}\left(Y_{2}\right)$ replaced by ${ }_{1} \mathcal{D} \preceq\left(\tilde{\mathcal{B}}^{5}\right),{ }_{1} \mathcal{D} \preceq\left(\tilde{\mathcal{B}}^{4}\right)$, see 3.15 . We shall take $\mathbf{X}$ in loc.cit. equal to $\Xi={ }^{\prime \prime} \vartheta_{!}\left({ }^{\prime \prime} K\right)$ as in $3.15,\left(w_{1}, w_{3}\right)=(u, w),\left(\lambda_{1}, \lambda_{3}\right)=\left(\lambda^{\prime}, \lambda\right)$. The conditions of loc.cit. are satisfied: those concerning $\mathbf{X}$ are satisfied with $c^{\prime}=k=$ $|w|+|u|+3 \nu+5 \rho$ (see 3.8(c)); those concerning $\Phi$ are satisfied with $c=a+\rho$ (see 3.17). We see that

$$
\begin{aligned}
& \underline{g r_{a+\rho+k}\left(\left({ }^{\prime \prime} d ! !^{\prime \prime} \vartheta_{!}\left({ }^{\prime \prime} K\right)\right)^{a+\rho+k}\right)((a+\rho+k) / 2)} \\
& =g r_{a+\rho}\left(\left({ }^{\prime \prime} d_{!} g r_{k}\left(\left({ }^{\prime \prime} \vartheta_{!}\left({ }^{\prime \prime} K\right)\right)^{k}\right)(k / 2)\right)^{a+\rho}\right)((a+\rho) / 2) .
\end{aligned}
$$

Using 3.11(a) we have:

$$
\begin{aligned}
\left.g r_{k}\left({ }^{\prime \prime} \vartheta_{!}\left({ }^{\prime \prime} K\right)\right)^{k}\right)(k / 2) & =\oplus_{y \in W} M_{\lambda^{\prime}, \lambda, \lambda, y(\lambda)}^{\dot{u}, \dot{y}, \dot{w}, \dot{y}^{-1},[1,4]}\langle 2|y|+|w|+|u|+5 \rho+\nu\rangle \\
& =\underline{g r_{k}\left({ }^{\prime \prime} \vartheta_{!}\left({ }^{\prime \prime} K\right)\right)^{k}}(k / 2) .
\end{aligned}
$$

Hence using $3.17(\mathrm{a})$ we have

$$
\begin{gathered}
\frac{g r_{a+\rho}\left(\left({ }^{\prime \prime} d_{!} g r_{k}\left(\left({ }^{\prime \prime} \vartheta_{!}\left({ }^{\prime \prime} K\right)\right)^{k}\right)(k / 2)\right)^{a+\rho}\right)}{((a+\rho) / 2)} \\
=\oplus_{y \in W} \oplus_{y^{\prime} \in W ; y^{\prime} \cdot \lambda \in \mathbf{c}} \operatorname{Hom}_{\mathcal{C}^{\mathrm{c}} \tilde{\mathcal{B}}^{2}}\left(\mathbf{L}_{\lambda}^{\dot{y}^{\prime}}, \mathbf{L}_{\lambda^{\prime}}^{\dot{\mathrm{o}}} \mathbf{L}_{\lambda}^{\dot{y}}\right) \\
\otimes M_{\lambda, \lambda, y(\lambda)}^{\dot{y}^{\prime}, \dot{w}, \dot{y}^{-1},[1,3]}\left\langle|y|+|w|+\left|y^{\prime}\right|+4 \rho+\nu\right\rangle .
\end{gathered}
$$

Since $y^{\prime} \cdot \lambda \in \mathbf{c}, u \cdot \lambda^{\prime} \in \mathbf{c}$, for $y \in W$ we have $\operatorname{Hom}_{\mathcal{C}^{\mathbf{c}} \tilde{\mathcal{B}}^{2}}\left(\mathbf{L}_{\lambda}^{\dot{y}^{\prime}}, \mathbf{L}_{\lambda^{\prime}}^{\dot{\dot{u}}} \mathbf{L}_{\lambda}^{\dot{y}}\right)=0$ 
unless $y \cdot \lambda \in \mathbf{c}($ see 2.26). Thus we have

$$
\begin{aligned}
& \frac{g r_{a+\rho+k}\left(\left({ }^{\prime \prime} d_{!}^{\prime \prime} \vartheta_{!}\left({ }^{\prime \prime} K\right)\right)^{a+\rho+k}\right)}{=}((a+\rho+k) / 2) \\
& \bigoplus_{y \in W ; y \cdot \lambda \in \mathbf{c}} \oplus_{y^{\prime} \in W ; y^{\prime} \cdot \lambda \in \mathbf{c}} \operatorname{Hom}_{\mathcal{C}^{\mathbf{c}} \tilde{\mathcal{B}}^{2}}\left(\mathbf{L}_{\lambda}^{\dot{y}^{\prime}}, \mathbf{L}_{\lambda^{\prime}}^{\dot{u}} \mathbf{L}_{\lambda}^{\dot{y}}\right) \\
& \otimes M_{\lambda, \lambda, y(\lambda)}^{\dot{y}^{\prime}, \dot{w}, \dot{y}^{-1},[1,3]}\left\langle|y|+|w|+\left|y^{\prime}\right|+4 \rho+\nu\right\rangle .
\end{aligned}
$$

The last Hom-space is zero unless $y(\lambda)=\lambda^{\prime}$ hence (with the change of notation $\left.\left(y, y^{\prime}\right) \mapsto\left(y^{\prime}, y\right)\right)$ :

$$
\begin{aligned}
& \frac{g r_{a+\rho+k}\left(\left(" d !^{\prime \prime} \vartheta_{!}\left({ }^{\prime \prime} K\right)\right)^{a+\rho+k}\right)}{=}((a+\rho+k) / 2) \\
& =\oplus_{y \in W ; y \cdot \lambda \in \mathbf{c}} \oplus_{y^{\prime} \in W ; y^{\prime} \cdot \lambda \in \mathbf{c}} \operatorname{Hom}_{\mathcal{C}^{\mathbf{c}} \tilde{\mathcal{B}}^{2}}\left(\mathbf{L}_{\lambda}^{\dot{y}}, \mathbf{L}_{\lambda^{\prime}}^{\dot{u}} \mathbf{L}_{\lambda}^{\dot{y}^{\prime}}\right) \\
& \otimes M_{\lambda, \lambda, \lambda^{\prime}}^{\dot{y}, \dot{w}, \dot{y}^{\prime}-1,[1,3]}\left\langle|y|+|w|+\left|y^{\prime}\right|+4 \rho+\nu\right\rangle .
\end{aligned}
$$

3.20. Let $y_{1} \cdot \lambda_{1} \in \mathbf{c}, y_{2} \cdot \lambda_{2} \in \mathbf{c}, y_{3} \cdot \lambda_{3} \in \mathbf{c}$. We show:

(a) We have canonically

$$
\operatorname{Hom}_{\mathcal{C}^{\mathbf{c}} \tilde{\mathcal{B}}^{2}}\left(\mathbf{L}_{y_{2}\left(\lambda_{2}\right)}^{\dot{y}_{2}^{-1}}, \mathbf{L}_{y_{1}\left(\lambda_{1}\right)}^{\dot{y}_{1}^{-1}}-\mathbf{L}_{\lambda_{3}}^{\dot{y_{3}}}\right)=\operatorname{Hom}_{\mathcal{C}^{\mathbf{c}} \tilde{\mathcal{B}}^{2}}\left(\mathbf{L}_{\lambda_{1}}^{\dot{y}_{1}}, \mathbf{L}_{\lambda_{3}}^{\dot{y}_{3}} \varrho \mathbf{L}_{\lambda_{2}}^{\dot{y}_{2}}\right)
$$

When $\lambda_{1} \neq \lambda_{2}$, both sides of the last equality (to be proved) are zero and the result is clear. In the rest of the proof we assume $\lambda_{1}=\lambda_{2}=\lambda$. We set $u \cdot \lambda^{\prime}=y_{3} \cdot \lambda_{3}$. Choose $w \in W$ such that $w \cdot \lambda \in W \mathfrak{s}$.

Applying 3.18(a), 3.19(a) to our $w \cdot \lambda, u \cdot \lambda^{\prime}$ and using the equality

$$
\begin{aligned}
& \frac{g r_{a+\rho+k}\left(\left({ }^{\prime} d_{!}^{\prime} \vartheta_{!}\left({ }^{\prime} K\right)\right)^{a+\rho+k}\right)}{}((a+\rho+k) / 2) \\
& \left.=\underline{g r_{a+\rho+k}\left(\left({ }^{\prime \prime} d_{!}^{\prime \prime} \vartheta_{!}\left({ }^{\prime \prime} K\right)\right)^{a}+\rho+k\right.}\right)((a+\rho+k) / 2)
\end{aligned}
$$

which comes from ' $d !{ }^{\prime} \vartheta_{!}\left({ }^{\prime} K\right)={ }^{\prime \prime} d !{ }^{\prime \prime} \vartheta_{!}\left({ }^{\prime \prime} K\right)$, see $3.15(\mathrm{a})$, we deduce

$$
\begin{aligned}
\oplus_{y \in W ; y \cdot \lambda \in \mathbf{c}} \oplus_{y^{\prime} \in W ; y^{\prime} \cdot \lambda \in \mathbf{c}} \operatorname{Hom}_{\mathcal{C}^{c} \tilde{\mathcal{B}}^{2}}\left(\mathbf{L}_{y^{\prime}(\lambda)}^{\dot{y}^{\prime}-1}, \mathbf{L}_{y(\lambda)}^{\dot{y}^{-1}} \mathbf{L}_{\lambda^{\prime}}^{\dot{u}}\right) \\
\quad \otimes M_{\lambda, \lambda, \lambda^{\prime}}^{\dot{y}, \dot{w}, \dot{y}^{\prime-1},[1,3]}\left\langle|y|+|w|+\left|y^{\prime}\right|+4 \rho+\nu\right\rangle \\
=\oplus_{y \in W ; y \cdot \lambda \in \mathbf{c}} \oplus_{y^{\prime} \in W ; y^{\prime} \cdot \lambda \in \mathbf{c}} \operatorname{Hom}_{\mathcal{C}^{\mathbf{c}} \tilde{\mathcal{B}}^{2}}\left(\mathbf{L}_{\lambda}^{\dot{y}}, \mathbf{L}_{\lambda^{\prime}}^{\dot{u}} \underline{\mathbf{L}_{\lambda}^{\dot{y}^{\prime}}}\right) \\
\otimes M_{\lambda, \lambda, \lambda^{\prime}}^{\dot{y}, \dot{w}, \dot{y}^{\prime-1},[1,3]}\left\langle|y|+|w|+\left|y^{\prime}\right|+4 \rho+\nu\right\rangle .
\end{aligned}
$$


Considering the coefficient of

$$
M_{\lambda, \lambda, \lambda^{\prime}}^{\dot{y}_{1}, \dot{w}, \dot{y}_{2}^{-1},[1,3]}\left\langle\left|y_{1}\right|+|w|+\left|y_{2}\right|+4 \rho+\nu\right\rangle
$$

in the two sides of (b) we obtain (a). From the proof one can see that the identification in (a) does not depend on the choice of $w$.

3.21. We assume that $w \cdot \lambda \in \breve{\mathbf{c}}, u \cdot \lambda^{\prime} \in \mathbf{c}$. We apply $p_{03}$ ! and $\langle N\rangle$ for some $N$ to the two sides of $3.20(\mathrm{~b})$. (Recall that $p_{03}: \tilde{\mathcal{B}}^{4} \rightarrow \tilde{\mathcal{B}}^{2}$.) We obtain

$$
\begin{aligned}
& \oplus_{y \in W ; y \cdot \lambda \in \mathbf{c}} \oplus_{y^{\prime} \in W ; y^{\prime} \cdot \lambda \in \mathbf{c}} \operatorname{Hom}_{\mathcal{C}^{\mathbf{c}} \tilde{\mathcal{B}}^{2}}\left(\mathbf{L}_{y^{\prime}(\lambda)}^{\dot{y}^{\prime-1}}, \mathbf{L}_{y(\lambda)}^{\dot{y}^{-1}} \mathbf{L}_{\lambda^{\prime}}^{\dot{u}}\right) \otimes \mathbf{L}_{\lambda}^{\dot{y}} \circ \mathbf{L}_{\lambda}^{\dot{w}} \circ \mathbf{L}_{y^{\prime}(\lambda)}^{\dot{y}^{\prime-1}} \\
& =\oplus_{y \in W ; y \cdot \lambda \in \mathbf{c}} \oplus_{y^{\prime} \in W ; y^{\prime} \cdot \lambda \in \mathbf{c}} \operatorname{Hom}_{\mathcal{C}^{\mathbf{c}} \tilde{\mathcal{B}}^{2}}\left(\mathbf{L}_{\lambda}^{\dot{y}}, \mathbf{L}_{\lambda^{\prime}}^{\dot{u}} \underline{\mathbf{L}_{\lambda}^{\prime}}\right) \otimes \mathbf{L}_{\lambda}^{\dot{y}} \circ \mathbf{L}_{\lambda}^{\dot{w}} \circ \mathbf{L}_{y^{\prime}(\lambda)}^{\dot{y}^{\prime}-1}
\end{aligned}
$$

(We have replaced $\mathbf{L}_{\lambda^{\prime}}^{\dot{y}^{\prime}-1}$ by $\mathbf{L}_{y^{\prime}(\lambda)}^{\dot{y}^{\prime-1}}$; in the last equality the terms with $\lambda^{\prime} \neq$

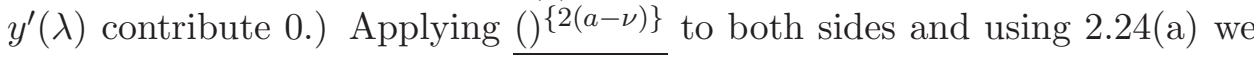
obtain

$$
\begin{aligned}
& \oplus_{y \in W ; y \cdot \lambda \in \mathbf{c}} \oplus_{y^{\prime} \in W ; y^{\prime} \cdot \lambda \in \mathbf{c}} \operatorname{Hom}_{\mathcal{C}^{\mathbf{c}} \tilde{\mathcal{B}}^{2}}\left(\mathbf{L}_{y^{\prime}(\lambda)}^{\dot{y}^{\prime-1}}, \mathbf{L}_{y(\lambda)}^{\dot{y}^{-1}} \underline{-} \mathbf{L}_{\lambda^{\prime}}^{\dot{u}}\right) \otimes \mathbf{L}_{\lambda}^{\dot{y}} \underline{\mathbf{L}_{\lambda}} \stackrel{\dot{w}}{{ }^{\prime}} \mathbf{L}_{y^{\prime}(\lambda)}^{\dot{y}^{\prime-1}} \\
& =\oplus_{y \in W ; y \cdot \lambda \in \mathbf{c}} \oplus_{y^{\prime} \in W ; y^{\prime} \cdot \lambda \in \mathbf{c}} \operatorname{Hom}_{\mathcal{C}^{\mathbf{c}} \tilde{\mathcal{B}}^{2}}\left(\mathbf{L}_{\lambda}^{\dot{y}}, \mathbf{L}_{\lambda^{\prime}}^{\dot{u}} \underline{\mathbf{L}_{\lambda}^{y^{\prime}}}\right) \otimes \mathbf{L}_{\lambda}^{\dot{y}} \underline{\mathbf{L}_{\lambda}} \underline{\mathbf{w}}_{\mathbf{L}^{\prime}(\lambda)}^{\dot{y}^{\prime-1}}
\end{aligned}
$$

or equivalently

$$
\oplus_{y \in W ; y \cdot \lambda \in \mathbf{c}} \mathbf{L}_{\lambda}^{\dot{y}} \underline{\underline{ }} \mathbf{L}_{\lambda}^{\dot{w} \varrho} \mathbf{L}_{y(\lambda)}^{\dot{y}^{-1}} \underline{-} \mathbf{L}_{\lambda^{\prime}}^{\dot{u}}=\oplus_{y^{\prime} \in W ; y^{\prime} \cdot \lambda \in \mathbf{c}} \mathbf{L}_{\lambda^{\prime}}^{\dot{\dot{u}}} \mathbf{L}_{\lambda}^{\dot{y}^{\prime}} \underline{\mathbf{L}_{\lambda}} \underline{\mathbf{w}}_{\mathrm{y}^{\prime}(\lambda)}^{\dot{y}^{\prime-1}} .
$$

Using 3.13(d), this can be rewritten as follows:

$$
\underline{\mathfrak{b}}\left(\mathbb{L}_{\lambda}^{\dot{w}}\right) \underline{\varrho} \mathbf{L}_{\lambda^{\prime}}^{\dot{u}}=\mathbf{L}_{\lambda^{\prime}}^{\dot{u}} \underline{\mathfrak{b}}\left(\mathbb{L}_{\lambda}^{\dot{w}}\right)
$$

Another identification of the two sides in (a) is given by $3.14(\mathrm{j})$ with $L=\mathbb{L}_{\lambda}^{\dot{w}}$, $L^{\prime}=\mathbf{L}_{\lambda^{\prime}}^{\dot{u}}\left(\right.$ note that $\underline{\mathfrak{b}}(L)=\underline{\mathfrak{b}}^{\prime}(L)$ by $\left.3.14(\mathrm{~d})\right)$. In fact, the arguments in 3.133.21 show that

(b) these two identifications of the two sides of (a) coincide.

3.22. Let

$$
\begin{aligned}
V=\{ & \left(B_{0}, B_{1}, B_{2}, g U_{B_{0}}, g^{\prime} U_{B_{1}}\right) ; \\
& \left.\left(B_{0}, B_{1}, B_{2}\right) \in \mathcal{B}^{3}, g \in G, g^{\prime} \in G, g B_{0} g^{-1}=B_{1}, g^{\prime} B_{1} g^{\prime-1}=B_{2}\right\} .
\end{aligned}
$$


Define $p_{01}: V \rightarrow Z, p_{12}: V \rightarrow Z, p_{02}: V \rightarrow Z$ by

$$
\begin{aligned}
& p_{01}:\left(B_{0}, B_{1}, B_{2}, g U_{B_{0}}, g^{\prime} U_{B_{1}}\right) \mapsto\left(B_{0}, B_{1}, g U_{B_{0}}\right), \\
& p_{12}:\left(B_{0}, B_{1}, B_{2}, g U_{B_{0}}, g^{\prime} U_{B_{1}}\right) \mapsto\left(B_{1}, B_{2}, g^{\prime} U_{B_{1}}\right), \\
& p_{02}:\left(B_{0}, B_{1}, B_{2}, g U_{B_{0}}, g^{\prime} U_{B_{1}}\right) \mapsto\left(B_{0}, B_{2}, g^{\prime} g U_{B_{0}}\right) .
\end{aligned}
$$

For $L, L^{\prime}$ in $\mathcal{D}(Z)$ we set

$$
L \bullet L^{\prime}=p_{02 !}\left(p_{01}^{*} L \otimes p_{12}^{*} L^{\prime}\right) \in \mathcal{D}(Z)
$$

This operation is associative. Hence if ${ }^{1} L,{ }^{2} L, \ldots,{ }^{r} L$ are in $\mathcal{D}(Z)$ then ${ }^{1} L$ ${ }^{2} L \bullet \ldots{ }^{r} L \in \mathcal{D}(Z)$ is well defined. We show:

(a) For $L, L^{\prime}$ in $\mathcal{D}(Z)$ we have canonically $\epsilon^{*}\left(L \bullet L^{\prime}\right)=\epsilon^{*}(L) \circ \epsilon^{*}\left(L^{\prime}\right)$.

Let

$$
Y=\left\{\left(x \mathbf{U}, y \mathbf{U}, g U_{x \mathbf{B} x^{-1}}\right) ; x \mathbf{U} \in \tilde{\mathcal{B}}, y \mathbf{U} \in \tilde{\mathcal{B}} ; g \in G\right\}
$$

Define $j: Y \rightarrow \tilde{\mathcal{B}}^{2}, j_{1}: Y \rightarrow Z, j_{2}: Y \rightarrow Z$ by

$$
\begin{aligned}
j\left(x \mathbf{U}, y \mathbf{U}, g U_{x} \mathbf{B} x^{-1}\right) & =(x \mathbf{U}, y \mathbf{U}), \\
j_{1}\left(x \mathbf{U}, y \mathbf{U}, g U_{x \mathbf{B} x^{-1}}\right) & =\left(x \mathbf{B} x^{-1}, g x \mathbf{B} x^{-1} g^{-1}, g U_{x \mathbf{B} x^{-1}}\right), \\
j_{2}\left(x \mathbf{U}, y \mathbf{U}, g U_{x} \mathbf{B} x^{-1}\right) & =\left(g x \mathbf{B} x^{-1} g^{-1}, y \mathbf{B} y^{-1}, y \mathbf{U} x^{-1} g^{-1}\right) .
\end{aligned}
$$

From the definitions we have

$$
\epsilon^{*}\left(L \bullet L^{\prime}\right)=j_{!}\left(j_{1}^{*}(L) \otimes j_{2}^{*}\left(L^{\prime}\right)\right)=\epsilon^{*}(L) \circ \epsilon^{*}\left(L^{\prime}\right)
$$

and (a) follows.

3.23. Let $L, L^{\prime} \in \mathcal{D}^{\boldsymbol{\top}} Z$. We show:

(a) If $L \in \mathcal{D}^{\preceq} Z$ or $L^{\prime} \in \mathcal{D}^{\preceq} Z$ then $L \bullet L^{\prime} \in \mathcal{D}^{\preceq} Z$. If $L \in \mathcal{D}^{\prec} Z$ or $L^{\prime} \in \mathcal{D}^{\prec} Z$ then $L \bullet L^{\prime} \in \mathcal{D}^{\prec} Z$.

For the first assertion of (a) we can assume that $L=\mathbb{L}_{\lambda}^{\dot{w}}, L^{\prime}=\mathbb{L}_{\lambda^{\prime}}^{\dot{w}^{\prime}}$ with $w \cdot \lambda, w^{\prime} \cdot \lambda^{\prime}$ in $\widetilde{W} \mathfrak{s}$ and either $w \cdot \lambda \preceq \mathbf{c}$ or $w^{\prime} \cdot \lambda^{\prime} \preceq \mathbf{c}$. Assume that 
$w_{1} \cdot \lambda_{1} \in \breve{W} \mathfrak{s}$ and $\mathbb{L}_{\lambda_{1}}^{\dot{w}_{1}}$ is a composition factor of $\left(L \bullet L^{\prime}\right)^{j}$. Then $\mathbf{L}_{\lambda_{1}}^{\dot{w}_{1}}=\tilde{\epsilon} \mathbb{L}_{\lambda_{1}}^{\dot{w}_{1}}$ is a composition factor of

$$
\begin{aligned}
\epsilon^{*}\left(L \bullet L^{\prime}\right)^{j}\langle\rho\rangle & =\left(\epsilon^{*}\left(L \bullet L^{\prime}\right)\right)^{j+\rho}(\rho / 2)=\left(\epsilon^{*} L \circ \epsilon^{*} L^{\prime}\right)^{j+\rho}(\rho / 2) \\
& =\left(\epsilon^{*} L\langle\rho\rangle \circ \epsilon^{*} L^{\prime}\langle\rho\rangle\right)^{j-\rho}(-\rho / 2)=\left(\mathbf{L}_{\lambda}^{\dot{w}} \circ \mathbf{L}_{\lambda^{\prime}}^{\dot{w}^{\prime}}\right)^{j-\rho}(\rho / 2) .
\end{aligned}
$$

From $2.23(\mathrm{~b})$ we see that $w_{1} \cdot \lambda_{1} \preceq \mathbf{c}$. This proves the first assertion of (a). The second assertion of (a) can be reduced to the first assertion.

(b) Assume that $L, L^{\prime} \in \mathcal{M}^{\wedge} Z$ and that either $L$ or $L^{\prime}$ is in $\mathcal{D}^{\preceq} Z$. If $j>$ $a+\rho-\nu$ then $\left(L \bullet L^{\prime}\right)^{j} \in \mathcal{M}^{\prec} Z$.

We can assume that $L=\mathbb{L}_{\lambda}^{\dot{w}}, L^{\prime}=\mathbb{L}_{\lambda^{\prime}}^{\dot{w}^{\prime}}$ with $w \cdot \lambda, w^{\prime} \cdot \lambda^{\prime}$ in $\breve{W} \mathfrak{s}$ and either $w \cdot \lambda \in \mathbf{c}$ or $w^{\prime} \cdot \lambda^{\prime} \in \mathbf{c}$. Assume that $w_{1} \cdot \lambda_{1} \in W \mathfrak{s}$ and $\mathbb{L}_{\lambda_{1}}^{\dot{w}_{1}}$ is a composition factor of $\left(L \bullet L^{\prime}\right)^{j}$. Then as in the proof of $(\mathrm{a}), \mathbf{L}_{\lambda_{1}}^{\dot{w}_{1}}$ is a composition factor of

$$
\tilde{e}\left(L \bullet L^{\prime}\right)^{j}=\left(\mathbf{L}_{\lambda}^{\dot{w}} \circ \mathbf{L}_{\lambda^{\prime}}^{\dot{w}^{\prime}}\right)^{j-\rho}(-\rho / 2)
$$

Since $j-\rho>a-\nu$ we see from $2.23\left(\right.$ a) that $w_{1} \cdot \lambda_{1} \prec \mathbf{c}$. This proves (b).

3.24. For $L, L^{\prime} \in \mathcal{C}_{0}^{\mathbf{c}} Z$ we set

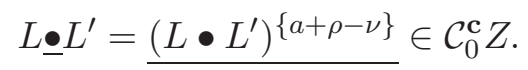

Using 3.23(a), (b) we see as in 2.24 that for $L, L^{\prime}, L^{\prime \prime} \in \mathcal{C}_{0}^{\mathbf{c}} Z$ we have

$$
L \bullet\left(L^{\prime} \bullet L^{\prime \prime}\right)=\left(L \bullet L^{\prime}\right) \underline{\bullet} L^{\prime \prime}=\underline{\left(L \bullet L^{\prime} \bullet L^{\prime \prime}\right)^{\{2 a+2 \rho-2 \nu\}}} .
$$

We see that $L, L^{\prime} \mapsto L \bullet L^{\prime}$ defines a monoidal structure on $\mathcal{C}_{0}^{\mathbf{c}} Z$. Hence if

$$
{ }^{1} L,{ }^{2} L, \ldots,{ }^{r} L
$$

are in $\mathcal{C}_{0}^{\mathbf{c}} Z$, then ${ }^{1} L \underline{\bullet}^{2} L \bullet \ldots \underline{\bullet}^{r} L \in \mathcal{C}_{0}^{\mathbf{c}} Z$ is well defined; we have

$$
{ }^{1} L \bullet^{2} L \bullet \ldots \bullet^{r} L=\underline{\left({ }^{1} L \bullet{ }^{2} L \bullet \ldots \bullet{ }^{r} L\right)^{\{(r-1)(a+\rho-\nu)\}}} .
$$

For $L, L^{\prime} \in \mathcal{C}_{0}^{\mathbf{c}} Z$ we have $\tilde{\epsilon} L, \tilde{\epsilon} L^{\prime} \in \mathcal{C}_{0}^{\mathbf{c}} \tilde{\mathcal{B}}^{2}$. We show:

$$
\tilde{\epsilon}\left(L \bullet L^{\prime}\right)=(\tilde{\epsilon} L) \underline{\circ}\left(\tilde{\epsilon} L^{\prime}\right) .
$$


It is enough to show that

$$
\begin{aligned}
& \epsilon^{*}\left(g r_{0}\left(\left(L \bullet L^{\prime}\right)^{a+\rho-\nu}\right)((a+\rho-\nu) / 2)\right)[\rho](\rho / 2) \\
& \left.\left.\quad=g r_{0}\left(\left(\epsilon^{*} L[\rho](\rho / 2) \circ \epsilon^{*} L^{\prime}[\rho](\rho / 2)\right)^{a-\nu}\right)((a-\nu) / 2)\right)\right) .
\end{aligned}
$$

The left hand side is equal to

$$
\left.g r_{0}\left(\epsilon^{*}\left(\left(L \bullet L^{\prime}\right)^{a+\rho-\nu}\right)((a+\rho-\nu) / 2)\right)[\rho](\rho / 2)\right)
$$

hence it is enough to show:

$$
\begin{aligned}
& \left.\epsilon^{*}\left(\left(L \bullet L^{\prime}\right)^{a+\rho-\nu}\right)((a+\rho-\nu) / 2)\right)[\rho](\rho / 2) \\
& \left.\quad=\left(\epsilon^{*} L[\rho](\rho / 2) \circ \epsilon^{*} L^{\prime}[\rho](\rho / 2)\right)^{a-\nu}((a-\nu) / 2)\right)
\end{aligned}
$$

that is,

$$
\epsilon^{*}\left(\left(L \bullet L^{\prime}\right)^{a+\rho-\nu}\right)[\rho]=\left(\epsilon^{*} L[\rho] \circ \epsilon^{*} L^{\prime}[\rho]\right)^{a-\nu},
$$

or, after using 3.3(b):

$$
\left(\epsilon^{*}\left(L \bullet L^{\prime}\right)\right)^{a+2 \rho-\nu}=\left(\epsilon^{*} L \circ \epsilon^{*} L^{\prime}\right)^{a+2 \rho-\nu} .
$$

It remains to use that $\epsilon^{*}\left(L \bullet L^{\prime}\right)=\epsilon^{*} L \circ \epsilon^{*} L^{\prime}$, see $3.22(\mathrm{a})$.

3.25. In the setup of 3.14 let

$$
\diamond \mathcal{Y}=\mathbf{T}^{2} \backslash\left\{\left(\left(x_{0} \mathbf{U}, x_{1} \mathbf{U}, x_{2} \mathbf{U}, x_{3} \mathbf{U}\right), g\right) \in \tilde{\mathcal{B}}^{4} \times G ; g \in x_{3} \mathbf{U} x_{0}^{-1}, g \in x_{2} \mathbf{U} x_{1}^{-1}\right\}
$$

where $\mathbf{T}^{2}$ acts freely by

$$
\left(t_{1}, t_{2}\right):\left(\left(x_{0} \mathbf{U}, x_{1} \mathbf{U}, x_{2} \mathbf{U}, x_{3} \mathbf{U}\right), g\right) \mapsto\left(\left(x_{0} t_{1} \mathbf{U}, x_{1} t_{2} \mathbf{U}, x_{2} t_{2} \mathbf{U}, x_{3} t_{1} \mathbf{U}\right), g\right) .
$$

We define ${ }^{\diamond} \eta: \diamond \mathcal{Y} \rightarrow Z$ by

$$
\left(\left(x_{0} \mathbf{U}, x_{1} \mathbf{U}, x_{2} \mathbf{U}, x_{3} \mathbf{U}\right), g\right) \mapsto \epsilon\left(x_{1} \mathbf{U}, x_{2} \mathbf{U}\right) .
$$

We define $d:{ }^{\diamond} \mathcal{Y} \rightarrow Z$ by

$$
\left(\left(x_{0} \mathbf{U}, x_{1} \mathbf{U}, x_{2} \mathbf{U}, x_{3} \mathbf{U}\right), g\right) \mapsto \epsilon\left(x_{0} \mathbf{U}, x_{3} \mathbf{U}\right)
$$


We define $\mathfrak{b}^{\prime \prime}: \mathcal{D}(Z) \rightarrow \mathcal{D}(Z)$ and $\mathfrak{b}^{\prime \prime}: \mathcal{D}_{m}(Z) \rightarrow \mathcal{D}_{m}(Z)$ by

$$
\mathfrak{b}^{\prime \prime}(L)=d_{!}\left({ }^{\diamond} \eta\right)^{*} L
$$

From the definitions it is clear that

$$
\mathfrak{b}^{\prime}(L)=\epsilon^{*} \mathfrak{b}^{\prime \prime}(L)
$$

Using (a) we see that 3.14(a), (b), (c) imply the following statements.

(b) If $L \in \mathcal{D}^{\preceq}(Z)$ then $\mathfrak{b}^{\prime \prime}(L) \in \mathcal{D}^{\preceq} Z$. If $L \in \mathcal{D}^{\prec}(Z)$ then $\mathfrak{b}^{\prime \prime}(L) \in \mathcal{D}^{\prec} Z$.

(c) If $L \in \mathcal{M} \preceq(Z)$ and $h>2 \nu+2 a$ then $\left(\mathfrak{b}^{\prime \prime}(L)\right)^{h} \in \mathcal{M}^{\prec} \tilde{\mathcal{B}}^{2}$.

We define $\underline{\mathfrak{b}^{\prime \prime}}: \mathcal{C}_{0}^{\mathbf{c}}(Z) \rightarrow \mathcal{C}_{0}^{\mathbf{c}}(Z)$ by

$$
\underline{\mathfrak{b}^{\prime \prime}}(L)=\underline{g r_{2 \nu+2 a}\left(\left(\mathfrak{b}^{\prime \prime}(L)\right)^{2 \nu+2 a}\right)}(\nu+a) .
$$

Using results in 3.3 we see that, if $L \in \mathcal{C}_{0}^{\mathbf{c}} Z$, then

(d) $\underline{\mathfrak{b}}^{\prime}(L)=\tilde{\epsilon}\left(\underline{\mathfrak{b}}^{\prime \prime}(L)\right)$.

\section{The Monoidal Category $\mathcal{C}^{\mathrm{c}} \tilde{\mathcal{B}}^{2}$ and Its Centre}

4.1. We consider the inclusion $\tilde{\mathcal{O}}_{1} \rightarrow \tilde{\mathcal{B}}^{2}$ where $\tilde{\mathcal{O}}_{1}=\left\{(x \mathbf{U}, y \mathbf{U}) \in \tilde{\mathcal{B}}^{2}, x^{-1} y \in\right.$ B $\}$. Let $w \cdot \lambda \in W \mathfrak{s}$ be such that $w \cdot \lambda \preceq \mathbf{c}$; let $i \in \mathbf{Z}$. From 2.12(a), (b) we deduce:

(a) $\quad \sum_{i \in \mathbf{Z}} \operatorname{rk}\left(\left.\mathcal{H}^{i} L_{\lambda}^{\dot{w} \sharp}\right|_{\tilde{\mathcal{O}}_{1}}\right) v^{i}$ is $v^{|w|} p_{1, w}^{\lambda}$ if $w \in W_{\lambda}$ and is 0 if $w \notin W_{\lambda}$;

where $p_{1, w}^{\lambda} \in \mathbf{Z}\left[v^{-1}\right]$ (as in 1.8) belongs to $v^{-a(w)} \mathbf{Z}\left[v^{-1}\right]$ (see [18, §14]) and $a(w)$ is the value at $w$ of the $a$-function of the Coxeter group $W_{\lambda}$ (so that $a(w) \geq a)$; moreover,

$$
\left.\mathcal{H}^{i} L_{\lambda}^{\dot{w} \sharp}\right|_{\tilde{\mathcal{O}}_{1}} \text { is a local system of pure weight } i \text {. }
$$

From (a) it follows that

$$
\sum_{i \in \mathbf{Z}} \operatorname{rk}\left(\left.\mathcal{H}^{i}\left(L_{\lambda}^{\dot{w} \sharp}[|w|]\right)\right|_{\tilde{\mathcal{O}}_{1}}\right) v^{i} \text { is in } v^{-a(w)} \mathbf{Z}\left[v^{-1}\right] \text { if } w \in W_{\lambda} \text { and is } 0 \text { if } w \notin W_{\lambda}
$$


so that

$$
\left.\left.\mathcal{H}^{i}\left(L_{\lambda}^{\dot{w} \sharp}[|w|]\right)\right|_{\tilde{\mathcal{O}}_{1}}\right) \text { is } 0 \text { if } i>-a .
$$

Define $\delta: \tilde{\mathcal{B}} \rightarrow \tilde{\mathcal{B}}^{2}$ by $x \mathbf{U} \mapsto(x \mathbf{U}, x \mathbf{U})$. Then the image of $d$ is contained in $\tilde{\mathcal{B}}_{1}$ and we deduce

(c) $\mathcal{H}^{i}\left(\delta^{*} L_{\lambda}^{\dot{w} \sharp}[|w|]\right)=0$ if $i>-a$.

We show:

(d) If $L \in \mathcal{M} \preceq \tilde{\mathcal{B}}^{2}$ and $j>-a-\rho$ then $\left(\delta^{*} L\right)^{j}=0$.

We can assume that $L=\mathbf{L}_{\lambda}^{\dot{w}}$ with $w \cdot \lambda$ as above. It is enough to show that for any $k$ we have $\left(\mathcal{H}^{k}\left(\delta^{*} L\right)[-k]\right)^{j}=0$ that is

$$
\left(\mathcal{H}^{k}\left(\delta^{*}\left(L_{\lambda}^{\dot{w} \sharp}[|w|+\nu+2 \rho]\right)\right)[\nu+\rho]\right)^{j-k-\nu-\rho}=0 .
$$

Now $\mathcal{H}^{k}\left(\delta^{*}\left(L_{\lambda}^{\dot{w} \sharp}[|w|+\nu+2 \rho]\right)\right)$ is a local system on $\tilde{\mathcal{B}}$ hence

$$
\mathcal{H}^{k}\left(\delta^{*}\left(L_{\lambda}^{\dot{w} \sharp}[|w|+\nu+2 \rho]\right)\right)[\nu+\rho]
$$

is a perverse sheaf on $\tilde{\mathcal{B}}$ so that we can assume that $j-k-\nu-\rho=0$. Thus it is enough to show that

$$
\mathcal{H}^{j-\nu-\rho}\left(\delta^{*}\left(L_{\lambda}^{\dot{w} \sharp}[|w|+\nu+2 \rho]\right)\right)=0
$$

or that $\mathcal{H}^{j+\rho}\left(\delta^{*}\left(L_{\lambda}^{\dot{w} \sharp}[|w|)\right)=0\right.$. This is indeed true by (c).

We show:

(e) If $L \in \mathcal{M} \frac{\prec}{m} \tilde{\mathcal{B}}^{2}$ is pure of weight 0 and $j \in \mathbf{Z}$ then $\left(\delta^{*} L\right)^{j}$ is pure of weight $j$.

We can assume that $L=\mathbf{L}_{\lambda}^{\dot{w}}$ with $\lambda, w$ as in (c). It is enough to prove that for any $k,\left(\mathcal{H}^{k}\left(\delta^{*} L\right)[-k]\right)^{j}$ is pure of weight $j$ that is,

$$
\left(\mathcal{H}^{k}\left(\delta^{*}\left(L_{\lambda}^{\dot{w} \sharp}[|w|+\nu+2 \rho]\right)\right)[\nu+\rho]\right)^{j-k-\nu-\rho}((|w|+\nu+2 \rho) / 2)
$$

is pure of weight $j$. As in the proof of (d) we can assume that $j-k-\nu-\rho=0$. Thus it is enough to show that

$$
\mathcal{H}^{j-\nu-\rho}\left(\delta^{*}\left(L_{\lambda}^{\dot{w} \sharp}[|w|+\nu+2 \rho]\right)\right)[\nu+\rho]((|w|+\nu+2 \rho) / 2)
$$


is pure of weight $j$ or that $\mathcal{H}^{j+|w|+\rho}\left(\delta^{*}\left(L_{\lambda}^{\dot{w} \sharp}\right)\right)\langle\nu+\rho\rangle((|w|+\rho) / 2)$ is pure of weight $j$. This follows from (b).

For $w \cdot \lambda \in \mathbf{c}$ we have as in the proof of (e):

$$
\left(\delta^{*} \mathbf{L}_{\lambda}^{\dot{w}}\right)^{-a-\rho}=\mathcal{H}^{-a+|w|}\left(\delta^{*}\left(L_{\lambda}^{\dot{w} \sharp}\right)\right)\langle\nu+\rho\rangle((|w|+\rho) / 2) .
$$

We set

$$
\beta_{w \cdot \lambda}=\mathcal{H}^{-a+|w|}\left(\delta^{*}\left(L_{\lambda}^{\dot{w} \sharp}\right)\right)((-a+|w|) / 2)
$$

This is a $G$-equivariant local system on $\tilde{\mathcal{B}}$ hence can be identified with a

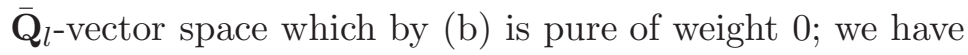

We show:

(f) $\operatorname{dim} \beta_{w \cdot \lambda}$ is 1 if $w \cdot \lambda \in \mathbf{D}_{\mathbf{c}}$ and is 0 if $w \cdot \lambda \notin \mathbf{D}_{\mathbf{c}}$. We have

$$
\left(\delta^{*} \mathbf{L}_{\lambda}^{\dot{w}}\right)^{-a-\rho}=\beta_{w \cdot \lambda}\langle\nu+\rho\rangle((a+\rho) / 2) .
$$

By (a), $\operatorname{dim} \beta_{w \cdot \lambda}$ is 0 if $w \notin W_{\lambda}$ while if $w \in W_{\lambda}$ it is equal to the coefficient of $v^{-a}$ in $p_{1, w}^{\lambda}$, which by [18, 14.2, P5] is 1 if $w$ is a distinguished involution of $W_{\lambda}$ and is 0 otherwise. This proves (f).

4.2. Let $\pi^{\prime}: \tilde{\mathcal{B}} \rightarrow \mathbf{p}$ be the obvious map. We show:

(a) Assume that $L \in \mathcal{M}_{m}(\tilde{\mathcal{B}})$ is $G$-equivariant so that $L=V \otimes \overline{\mathbf{Q}}_{l}\langle\nu+\rho\rangle$ where $V$ is a mixed vector space. If $j>\nu+\rho$ then $\left(\pi_{!}^{\prime} L\right)^{j}=0$.

We have $\mathcal{H}^{j}\left(p_{!}^{\prime} L\right)=V \otimes H_{c}^{j+\nu+\rho}\left(\tilde{\mathcal{B}}, \overline{\mathbf{Q}}_{l}\right)((\nu+\rho) / 2)$. This is zero if $j+\nu+\rho>$ $2 \nu+2 \rho$ since $\tilde{\mathcal{B}}$ is irreducible of dimension $\nu+\rho$.

We show:

(b) If $L \in \mathcal{M} \frac{\prec}{\bar{m}} \tilde{\mathcal{B}}^{2}$ and $j>\nu-a$ then $\left(\pi_{!}^{\prime} \delta^{*} L\right)^{j}=0$ (with $\delta$ as in 4.1). Moreover we have canonically $\left(\pi_{!}^{\prime} \delta^{*} L\right)^{\nu-a}=\left(\pi_{!}^{\prime}\left(\left(\delta^{*} L\right)^{-a-\rho}\right)\right)^{\nu+\rho}$.

The proof has much in common with that of [24, 8.2].

Let $\mathbf{X}=\delta^{*} L$. For any $i$ we have a distinguished triangle $\left(\tau_{<i} \mathbf{X}, \tau_{\leq i} \mathbf{X}\right.$, $\left.\mathbf{X}^{i}[-i]\right)$ where we write $\tau_{<i}, \tau_{\leq i}$ for what in [1] is denoted by ${ }^{p} \tau_{<i},{ }^{p} \tau_{\leq i}$. 
We deduce a distinguished triangle $\left(\pi_{!}^{\prime}\left(\tau_{<i} \mathbf{X}\right), \pi_{!}^{\prime}\left(\tau_{\leq i} \mathbf{X}\right), \pi_{!}^{\prime}\left(\mathbf{X}^{i}\right)[-i]\right)$ hence an exact sequence

$$
\begin{aligned}
\left(\pi_{!}^{\prime}\left(\mathbf{X}^{i}\right)\right)^{h-1} & \rightarrow\left(\pi_{!}^{\prime}\left(\tau_{<i} \mathbf{X}\right)\right)^{i+h} \rightarrow\left(\pi_{!}^{\prime}\left(\tau_{\leq i} \mathbf{X}\right)\right)^{i+h} \rightarrow\left(\pi_{!}^{\prime}\left(\mathbf{X}^{i}\right)\right)^{h} \\
& \rightarrow\left(\pi_{!}^{\prime}\left(\tau_{<i} \mathbf{X}\right)\right)^{i+h+1}
\end{aligned}
$$

We show by induction on $i$ that

(d) $\left(\pi_{!}^{\prime}\left(\tau_{\leq i} \mathbf{X}\right)\right)^{j}=0$ for $j>\nu-a$.

For sufficiently negative $i,(\mathrm{~d})$ is obvious. Thus we can assume that $(\mathrm{d})$ is known when $i$ is replaced by $i-1$. From (c) with $h=j-i$ we see using the induction hypothesis that we have an exact sequence $0 \rightarrow\left(\pi_{!}^{\prime}\left(\tau_{\leq i} \mathbf{X}\right)\right)^{j} \rightarrow$ $\left(\pi_{!}^{\prime}\left(\mathbf{X}^{i}\right)\right)^{j-i}$. It is then enough to show that $\left(\pi_{!}^{\prime}\left(\mathbf{X}^{i}\right)\right)^{j-i}=0$. If $i>-a-\rho$ then $\mathbf{X}^{i}=0$ by $4.1(\mathrm{~d})$. Thus we can assume that $i \leq-a-\rho$ so that $j-i>\nu+\rho$ and the equality $\left(\pi_{!}^{\prime}\left(\mathbf{X}^{i}\right)\right)^{j-i}=0$ follows from (a) with $L=\mathbf{X}^{i}$. This proves $(d)$.

In particular the first assertion of (b) holds. We now take $h=\nu-a-i$ in (c). Assuming that $i>-a-\rho$ we obtain (using 4.1(d))

$$
\left(\pi_{!}^{\prime}\left(\tau_{<i} \mathbf{X}\right)\right)^{\nu-a} \stackrel{\sim}{\rightarrow}\left(\pi_{!}^{\prime}\left(\tau_{\leq i} \mathbf{X}\right)\right)^{\nu-a}
$$

Hence

$$
\left(\pi_{!}^{\prime}\left(\tau_{\leq-a-\rho} \mathbf{X}\right)\right)^{\nu-a} \stackrel{\sim}{\rightarrow}\left(\pi_{!}^{\prime} \mathbf{X}\right)^{\nu-a}
$$

We show by induction on $i$ :

(e) $\left(\pi_{!}^{\prime}\left(\tau_{\leq i} \mathbf{X}\right)\right)^{j}=0$ if $i<-a-\rho, j=\nu-a$.

For sufficiently negative $i,(\mathrm{e})$ is obvious. Thus we can assume that (e) is known when $i$ is replaced by $i-1$. From (c) with $h=j-i$ we see using the induction hypothesis that we have an exact sequence $0 \rightarrow\left(\pi_{!}^{\prime}\left(\tau_{\leq i} \mathbf{X}\right)\right)^{j} \rightarrow$ $\left(\pi_{!}^{\prime}\left(\mathbf{X}^{i}\right)\right)^{j-i}$. It is then enough to show that $\left(\pi_{!}^{\prime}\left(\mathbf{X}^{i}\right)\right)^{j-i}=0$. We have $j-i>\nu+\rho$ and the equality $\left(\pi_{!}^{\prime}\left(\mathbf{X}^{i}\right)\right)^{j-i}=0$ follows from (a) with $L=\mathbf{X}^{i}$. This proves (e).

From (e) we see that $\left(\pi_{!}\left(\tau_{\leq-a-\rho-1} \mathbf{X}\right)\right)^{\nu-a}=0$. From (c) with $i=-a-\rho$, $h=\nu+\rho$ we deduce an exact sequence

$$
0 \rightarrow\left(\pi_{!}^{\prime} \mathbf{X}\right)^{\nu-a} \rightarrow\left(\pi_{!}^{\prime}\left(\mathbf{X}^{-a-\rho}\right)\right)^{\nu+\rho} \rightarrow 0
$$


This completes the proof of (b).

We show:

(f) If $L \in \mathcal{C}_{0}^{\mathbf{c}} \tilde{\mathcal{B}}^{2}$ then $\left(\pi_{!}^{\prime}\left(\left(\delta^{*} L\right)^{-a-\rho}\right)\right)^{\nu+\rho}$ and $\left(\pi_{!}^{\prime} \delta^{*} L\right)^{\nu-a}$ are pure of weight $\nu-a$.

We can assume that $L=\mathbf{L}_{\lambda}^{\dot{w}}$ where $w \cdot \lambda \in \mathbf{c}$. Using $4.1(\mathrm{f})$ we have

$$
\begin{aligned}
& \left(\pi_{!}^{\prime}\left(\left(\delta^{*} L\right)^{-a-\rho}\right)\right)^{\nu+\rho}((-a+\nu) / 2) \\
& =\left(\pi_{!}^{\prime}\left(\beta_{w \cdot \lambda}\langle\nu+\rho\rangle\right)((a+\rho) / 2)\right)^{\nu+\rho}((-a+\nu) / 2) \\
& =\beta_{w \cdot \lambda} \otimes\left(\pi_{!}^{\prime} \overline{\mathbf{Q}}_{l}\langle\nu+\rho\rangle\right)^{\nu+\rho}((\nu+\rho) / 2) \\
& =\beta_{w \cdot \lambda} \otimes\left(\pi_{!}^{\prime} \overline{\mathbf{Q}}_{l}\right)^{2 \nu+2 \rho}(\nu+\rho)=\beta_{w \cdot \lambda .}
\end{aligned}
$$

Since $b_{w \cdot \lambda}$ is pure of weight 0 , we see that $\left(\pi_{!}^{\prime}\left(\left(\delta^{*} L\right)^{-a-\rho}\right)\right)^{\nu+\rho}$ is pure of weight $\nu-a$. Using (b) we deduce that $\left(\pi_{!}^{\prime} \delta^{*} L\right)^{\nu-a}$ is pure of weight $\nu-a$.

4.3. We set

$$
\mathbf{1}^{\prime}=\oplus_{d \cdot \lambda \in \mathbf{D}_{\mathbf{c}}} \beta_{d \cdot \lambda}^{*} \otimes \mathbf{L}_{\lambda}^{\dot{d}} \in \mathcal{C}_{0}^{\mathbf{c}} \tilde{\mathcal{B}}^{2}
$$

Here $\beta_{d \cdot \lambda}^{*}$ is the vector space dual to $\beta_{d \cdot \lambda}$, see $4.1(\mathrm{f})$. For $L \in \mathcal{C}^{\mathbf{c}} \tilde{\mathcal{B}}^{2}$ we show

$$
\operatorname{Hom}_{\mathcal{C}^{\mathbf{c}} \tilde{\mathcal{B}}^{2}}\left(\mathbf{1}^{\prime}, L\right)=\left(\pi_{!}^{\prime}\left(\left(\delta^{*} L\right)^{-a-\rho}\right)\right)^{\nu+\rho}((-a+\nu) / 2) .
$$

We can assume that $L=\mathbf{L}_{\lambda}^{\dot{w}}$ where $w \cdot \lambda \in \mathbf{c}$. Assume first that $w \cdot \lambda \in \mathbf{D}_{\mathbf{c}}$. Then $\operatorname{Hom}_{\mathcal{C}^{\mathrm{c}} \tilde{\mathcal{B}}^{2}}\left(\mathbf{1}^{\prime}, L\right)=\beta_{w \cdot \lambda}$ and (as in the proof of $4.2(\mathrm{f})$ ):

$$
\left(\pi_{!}^{\prime}\left(\left(\delta^{*} L\right)^{-a-\rho}\right)\right)^{\nu+\rho}((-a+\nu) / 2)=\beta_{w \cdot \lambda} .
$$

Thus (a) holds in this case. Next assume that $w \cdot \lambda \notin \mathbf{D}_{\mathbf{c}}$. Then both sides of (a) are zero (we use 4.1(f)).

4.4. For $L \in \mathcal{D}_{m}\left(\tilde{\mathcal{B}}^{2}\right)$ we set $L^{\dagger}=\tilde{\mathfrak{h}}^{*} L$ where $\tilde{\mathfrak{h}}: \tilde{\mathcal{B}}^{2} \rightarrow \tilde{\mathcal{B}}^{2}$ is as in 2.1. Let $\pi^{\prime \prime}: \tilde{\mathcal{B}}^{2} \rightarrow \mathbf{p}$ be the obvious map. For $L, L^{\prime}$ in $\mathcal{D}_{m}\left(\tilde{\mathcal{B}}^{2}\right)$ we have from the definitions

$$
\pi_{!}^{\prime} \delta^{*}\left(L \circ L^{\prime}\right)=\pi_{!}^{\prime \prime}\left(L \otimes L^{\prime \dagger}\right)
$$

We show: 
(b) If $L \in \mathcal{C}_{0}^{\mathbf{c}} \tilde{\mathcal{B}}^{2}$ then $\mathfrak{D}\left(L^{\dagger}\right) \in \mathcal{C}_{0}^{\mathbf{c}} \tilde{\mathcal{B}}^{2}$.

We can assume that $L=\mathbf{L}_{\lambda}^{\dot{w}}$ where $w \cdot \lambda \in \mathbf{c}$. Using $2.2($ a) and the definitions we have

$$
\mathfrak{D}\left(L^{\dagger}\right)=\mathfrak{D}\left(\mathbf{L}_{w\left(\lambda^{-1}\right)}^{\dot{w}^{-1}}\right)=\mathbf{L}_{w(\lambda)}^{\dot{w}^{-1}}
$$

It remains to use that $w^{-1} \cdot w(\lambda) \in \mathbf{c}$, by property Q10 in 1.9 for $\mathbf{H}$.

4.5. We show:

(a) For $L, L^{\prime}$ in $\mathcal{C}^{\mathbf{c}} \tilde{\mathcal{B}}^{2}$ we have canonically

$$
\operatorname{Hom}_{\mathcal{C}^{\mathbf{c}} \tilde{\mathcal{B}}^{2}}\left(\mathbf{1}^{\prime}, L \underline{-} L^{\prime}\right)=\operatorname{Hom}_{\mathcal{C}^{\mathbf{c}} \tilde{\mathcal{B}}^{2}}\left(\mathfrak{D}\left(L^{\prime \dagger}\right), L\right) .
$$

We can assume that $L=\mathbf{L}_{\lambda}^{\dot{w}}, L^{\prime}=\mathbf{L}_{\lambda^{\prime}}^{\dot{w}^{\prime}}$ where $w \cdot \lambda \in \mathbf{c}, w^{\prime} \cdot \lambda^{\prime} \in \mathbf{c}$. Using 4.3(a) and 4.2(b), 4.2(f), we have

$$
\begin{gathered}
\operatorname{Hom}_{\mathcal{C}^{\mathbf{c}} \tilde{\mathcal{B}}^{2}}\left(\mathbf{1}^{\prime}, L \underline{\circ} L^{\prime}\right)=\left(\pi_{!}^{\prime}\left(\left(\delta^{*}\left(L \_L^{\prime}\right)\right)^{-a-\rho}\right)\right)^{\nu+\rho}((-a+\nu) / 2) \\
=\left(\pi_{!}^{\prime} \delta^{*}\left(L \underline{\circ} L^{\prime}\right)\right)^{\nu-a}((-a+\nu) / 2)=\left(\pi_{!}^{\prime} \delta^{*}\left(L \varrho \underline{o} L^{\prime}\right)\right)^{\{\nu-a\}} .
\end{gathered}
$$

Applying [24, 8.2] with $\Phi: \mathcal{D}_{\bar{m}}^{\prec} Z \rightarrow \mathcal{D}_{\mathbf{p}}, \tilde{L} \mapsto \pi_{!}^{\prime} \delta^{*} \tilde{L}, c=\nu-a, c^{\prime}=-\nu+a$ (see $4.2(\mathrm{~b})$ ) we see that we have canonically

$$
\left(\pi_{!}^{\prime} \delta^{*}\left(L \underline{\circ} L^{\prime}\right)\right)^{\{\nu-a\}} \subset\left(\pi_{!}^{\prime} \delta^{*}\left(L \bullet L^{\prime}\right)\right)^{\{0\}} .
$$

From [15, 7.4] we see that we have canonically

$$
\left(\pi_{!}^{\prime \prime}\left(L \otimes L^{\prime \dagger}\right)\right)^{0}=\left(\pi_{!}^{\prime \prime}\left(L \otimes L^{\prime \dagger}\right)\right)^{\{0\}}=\operatorname{Hom}_{\mathcal{D}\left(\tilde{\mathcal{B}}^{2}\right)}\left(\mathfrak{D}\left(L^{\prime \dagger}\right), L\right) .
$$

By 4.4(a) we have $\pi_{!}^{\prime} \delta^{*}\left(L \bullet L^{\prime}\right)=\pi_{!}^{\prime \prime}\left(L \otimes L^{\prime \dagger}\right)$. Hence by combining (b), (c), (d) we have

$$
\operatorname{Hom}_{\mathcal{C}^{\mathbf{c}} \tilde{\mathcal{B}}^{2}}\left(\mathbf{1}^{\prime}, L_{\underline{O}} L^{\prime}\right) \subset \operatorname{Hom}_{\mathcal{C}^{\mathbf{c}} \tilde{\mathcal{B}}^{2}}\left(\mathfrak{D}\left(L^{\prime \dagger}\right), L\right)
$$

The dimension of the left hand side of (e) is the sum over $z \cdot \lambda_{1} \in \mathbf{D}_{\mathbf{c}}$ of the coefficient of $t_{z \cdot \lambda_{1}}$ in $t_{w \cdot \lambda} t_{w^{\prime} \cdot \lambda^{\prime}} \in \mathbf{H}^{\infty}$ and by the properties Q1,Q2, Q4 (in 1.9) of $\mathbf{H}$, this sum is equal to 1 if $w^{\prime} \cdot \lambda^{\prime}=w^{-1} \cdot w(\lambda)$ and is 0 if $w^{\prime} \cdot \lambda^{\prime} \neq w^{-1} \cdot w(\lambda)$; hence it is equal to the dimension of the right hand side of (e). It follows that (e) is an equality and (a) follows. 
4.6. The bifunctor $\mathcal{C}_{0}^{\mathrm{c}} \tilde{\mathcal{B}}^{2} \times \mathcal{C}_{0}^{\mathrm{c}} \tilde{\mathcal{B}}^{2} \rightarrow \mathcal{C}_{0}^{\mathrm{c}} \tilde{\mathcal{B}}^{2}, L, L^{\prime} \mapsto L \underline{\circ} L^{\prime}$ in 2.24 gives rise to a bifunctor $\mathcal{C}^{\mathbf{c}} \tilde{\mathcal{B}}^{2} \times \mathcal{C}^{\mathbf{c}} \tilde{\mathcal{B}}^{2} \rightarrow \mathcal{C}^{\mathbf{c}} \tilde{\mathcal{B}}^{2}$ denoted again by $L, L^{\prime} \mapsto L \underline{\text { o }} L^{\prime}$ as follows. Let $L \in \mathcal{C}^{\mathbf{c}} \tilde{\mathcal{B}}^{2}, L^{\prime} \in \mathcal{C}^{\mathbf{c}} \tilde{\mathcal{B}}^{2}$; we choose mixed structures of pure weight 0 on $L, L^{\prime}$, we define $L \_L^{\prime}$ as in 2.24 in terms of these mixed structures and we then disregard the mixed structure on $L \_L^{\prime}$. The resulting object of $\mathcal{C}^{\mathbf{c}} \tilde{\mathcal{B}}^{2}$ is denoted again by $L \_L^{\prime}$; it is independent of the choices made.

Similarly the bifunctor $\mathcal{C}_{0}^{\mathbf{c}} Z \times \mathcal{C}_{0}^{\mathbf{c}} Z \rightarrow \mathcal{C}_{0}^{\mathbf{c}} Z, L, L^{\prime} \mapsto L \bullet L^{\prime}$ in 3.24 gives rise to a bifunctor $\mathcal{C}^{\mathbf{c}} Z \times \mathcal{C}^{\mathbf{c}} Z \rightarrow \mathcal{C}^{\mathbf{c}} Z$ denoted again by $L, L^{\prime} \mapsto L \bullet L^{\prime}$.

The operation $L \bullet L^{\prime}\left(\right.$ resp. $\left.L \_-L^{\prime}\right)$ makes $\mathcal{C}^{\mathbf{c}} Z$ (resp. $\left.\mathcal{C}^{\mathbf{c}} \tilde{\mathcal{B}}^{2}\right)$ into a monoidal abelian category (see 2.24, 3.24).

The following result can be deduced from 2.22(c).

(a) Let $w_{i} \cdot \lambda_{i} \in \mathbf{c}, i=1,2$. In $\mathcal{C}^{\mathbf{c}} \tilde{\mathcal{B}}^{2}$ we have

$$
\mathbf{L}_{\lambda_{1}}^{\dot{w}_{1}} \varrho \mathbf{L}_{\lambda_{2}}^{\dot{w}_{2}} \cong \oplus_{w \cdot \lambda \in \mathbf{c}}\left(\mathbf{L}_{\lambda}^{\dot{w}}\right)^{\oplus f(w \cdot \lambda)}
$$

where $f(w \cdot \lambda) \in \mathbf{N}$ are given by

$$
t_{w_{1} \cdot \lambda_{1}} t_{w_{2} \cdot \lambda_{2}}=\sum_{w \cdot \lambda \in \mathbf{c}} f(w \cdot \lambda) t_{w \cdot \lambda} \in \mathbf{H}_{\mathbf{c}}^{\infty} .
$$

It follows that:

(b) in (a) we have $f(w \cdot \lambda)=0$ unless $\lambda=\lambda_{2}, w(\lambda)=w_{1}\left(\lambda_{1}\right), \lambda_{1}=w_{2}\left(\lambda_{2}\right)$.

To see this, we use that, setting for any $\lambda \in \mathfrak{o}$ :

$1_{\lambda}^{\mathbf{c}}=\sum_{d} t_{d \cdot \lambda} \in \mathbf{H}_{\mathbf{c}}^{\infty}$ (sum over all distinguished involutions $d$ of $W_{\lambda}$ ), we have $1_{\lambda}^{\mathrm{c}} 1_{\lambda^{\prime}}^{\mathrm{c}}=\delta_{\lambda, \lambda^{\prime}} 1_{\lambda}^{\mathrm{c}}$ for $\lambda, \lambda^{\prime}$ in $\mathfrak{o}$ and

$$
t_{w \cdot \lambda}=t_{w \cdot \lambda} 1_{\lambda}^{\mathbf{c}}=1_{w(l)}^{\mathbf{c}} t_{w \cdot \lambda} \text { for any } w \cdot \lambda \in \mathbf{c} .
$$

For any $\lambda_{1}, \lambda_{2}$ in o let $\mathcal{C}_{\lambda_{1}, \lambda_{2}}^{\mathbf{c}} \tilde{\mathcal{B}}^{2}$ be the subcategory of $\mathcal{C}^{\mathbf{c}} \tilde{\mathcal{B}}^{2}$ consisting of objects which are direct sums of objects of the form $\mathbf{L}_{\lambda_{2}}^{\dot{w}}$ for various $w \in W$ such that $w\left(\lambda_{2}\right)=\lambda_{1}$ and $w \cdot \lambda_{2} \in \mathbf{c}$. Clearly, any object $L \in \mathcal{C}^{\mathbf{c}} \tilde{\mathcal{B}}^{2}$ is canonically of the form

(c) $\oplus_{\lambda_{1}, \lambda_{2} \in \mathfrak{o}} L_{\lambda_{1}, \lambda_{2}}$ where $L_{\lambda_{1}, \lambda_{2}} \in \mathcal{C}_{\lambda_{1}, \lambda_{2}}^{\mathbf{c}}$.

From (b) we see that 
(d) if $\lambda_{1}, \lambda_{2}, \lambda_{3}, \lambda_{4}$ are elements of $\mathfrak{o}$ and $L \in \mathcal{C}_{\lambda_{1}, \lambda_{2}}^{\mathbf{c}}, L^{\prime} \in \mathcal{C}_{\lambda_{3}, \lambda_{4}}^{\mathbf{c}}$ then $L \underline{\circ} L^{\prime} \in$ $\mathcal{C}_{\lambda_{1}, \lambda_{4}}^{\mathbf{c}} ;$ moreover, Lo $L^{\prime}=0$ unless $\lambda_{2}=\lambda_{3}$.

4.7. We set

$$
\mathbf{1}=\oplus_{d \cdot \lambda \in \mathbf{D}_{\mathbf{c}}} \beta_{d \cdot \lambda} \otimes \mathbf{L}_{\lambda}^{(\dot{d})^{-1}} \in \mathcal{C}_{0}^{\mathbf{c}} \tilde{\mathcal{B}}^{2}
$$

Here $\beta_{d \cdot \lambda}$ is as in $4.1(\mathrm{f})$.

Let $y_{2} \cdot \lambda_{2} \in \mathbf{c}, y_{3} \cdot \lambda_{3} \in \mathbf{c}$. From $3.20\left(\right.$ a) we have for any $d \cdot \lambda \in \mathbf{D}_{\mathbf{c}}$ :

$$
\operatorname{Hom}_{\mathcal{C}^{\mathbf{c}} \tilde{\mathcal{B}}^{2}}\left(\mathbf{L}_{y_{2}\left(\lambda_{2}\right)}^{\dot{y}_{2}^{-1}}, \mathbf{L}_{\lambda}^{(\dot{d})^{-1}} \stackrel{\circ}{\mathbf{L}_{\lambda_{3}}^{\dot{y}_{3}}}\right)=\operatorname{Hom}_{\mathcal{C}^{\mathrm{c}} \tilde{\mathcal{B}}^{2}}\left(\mathbf{L}_{\lambda}^{\dot{d}}, \mathbf{L}_{\lambda_{3}}^{\dot{y_{3}} \varrho \mathbf{L}_{\lambda_{2}}^{\dot{y}_{2}}}\right) .
$$

It follows that

$$
\operatorname{Hom}_{\mathcal{C}^{\mathbf{c}} \tilde{\mathcal{B}}^{2}}\left(\mathbf{L}_{y_{2}\left(\lambda_{2}\right)}^{\dot{y}_{2}^{-1}}, \mathbf{1} \_\mathbf{L}_{\lambda_{3}}^{\dot{y_{3}}}\right)=\operatorname{Hom}_{\mathcal{C}^{\mathbf{c}} \tilde{\mathcal{B}}^{2}}\left(\mathbf{1}^{\prime}, \mathbf{L}_{\lambda_{3}}^{\dot{y}_{3}} \circ \mathbf{L}_{\lambda_{2}}^{\dot{y}_{2}}\right) .
$$

From 4.5(a) we have:

$$
\operatorname{Hom}_{\mathcal{C}^{c} \tilde{\mathcal{B}}^{2}}\left(\mathbf{1}^{\prime}, \mathbf{L}_{\lambda_{3} \varrho}^{\dot{y}_{3}} \mathbf{L}_{\lambda_{2}}^{\dot{y}_{2}}\right)=\operatorname{Hom}_{\mathcal{C}^{c} \tilde{\mathcal{B}}^{2}}\left(\mathbf{L}_{y_{2}\left(\lambda_{2}\right)}^{\dot{y}_{2}^{-1}}, \mathbf{L}_{\lambda_{3}}^{\dot{y_{3}}}\right)
$$

(We have used 2.2(a) and the equality

$$
\mathfrak{D}\left(\mathbf{L}_{\lambda}^{\omega}\right)=\mathbf{L}_{\lambda^{-1}}^{\omega}
$$

for any $w \cdot \lambda \in W \mathfrak{s}$ and $\omega \in \kappa_{q}^{-1}(w)$.) Using this, (b) becomes

$$
\operatorname{Hom}_{\mathcal{C}^{\mathbf{c}} \tilde{\mathcal{B}}^{2}}\left(\mathbf{L}_{y_{2}\left(\lambda_{2}\right)}^{\dot{y}_{2}^{-1}}, \mathbf{1}_{\mathbf{O}} \mathbf{L}_{\lambda_{3}}^{\dot{y_{3}}}\right)=\operatorname{Hom}_{\mathcal{C}^{\mathbf{c}} \tilde{\mathcal{B}}^{2}}\left(\mathbf{L}_{y_{2}\left(\lambda_{2}\right)}^{\dot{y}_{2}^{-1}}, \mathbf{L}_{\lambda_{3}}^{\dot{y_{3}}}\right) .
$$

Since this holds for any $y_{2} \cdot \lambda_{2} \in \mathbf{c}$, we see that we have canonically

$$
\mathbf{1} \_\mathbf{L}_{\lambda_{3}}^{\dot{y}_{3}}=\mathbf{L}_{\lambda_{3}}^{\dot{y}_{3}} \text {. }
$$

Since this holds for any $y_{3} \cdot \lambda_{3} \in \mathbf{c}$, we see that we have canonically

$$
\text { 1} \_L=L \text { for any } L \in \mathcal{C}_{0}^{\mathbf{c}} \tilde{\mathcal{B}}^{2}
$$

for any $L$ in $\mathcal{C}^{\mathbf{c}} \tilde{\mathcal{B}}^{2}$. Now $\mathcal{C}^{\mathbf{c}} \tilde{\mathcal{B}}^{2} \rightarrow \mathcal{C}^{\tilde{\mathbf{c}}} \tilde{\mathcal{B}}^{2}, L \mapsto L^{\dagger}$, satisfies

$$
\left(L \underline{\circ} L^{\prime}\right)^{\dagger}=L^{\prime \dagger} \underline{\circ} L^{\dagger}
$$


for any $L, L^{\prime}$ in $\mathcal{C}^{\mathbf{c}} \tilde{\mathcal{B}}^{2}$. Applying $L \mapsto \mathfrak{D}\left(L^{\dagger}\right)$ to (d) and using (e) and 2.25(a) we get

$$
L \circ \mathfrak{D}\left(\mathbf{1}^{\dagger}\right)=L \text { for any } L \in \mathcal{C}_{0}^{\mathbf{c}} \tilde{\mathcal{B}}^{2} .
$$

From (d), (f) we deduce that we have canonically $\mathbf{1}=\mathbf{1}$ ㅇD $\left(\mathbf{1}^{\dagger}\right)=\mathfrak{D}\left(\mathbf{1}^{\dagger}\right)$. Using 4.4(c) we see that $\mathfrak{D}\left(\mathbf{1}^{\prime \dagger}\right)=\mathbf{1}$ hence $\mathfrak{D}\left(\mathbf{1}^{\dagger}\right)=\mathbf{1}^{\prime}$. We see that

(g) $\mathbf{1}=\mathbf{1}^{\prime}=\mathfrak{D}\left(\mathbf{1}^{\dagger}\right)$ is a unit object of the monoidal category $\mathcal{C}^{\mathbf{c}} \tilde{\mathcal{B}}^{2}$.

4.8. For $L \in \mathcal{C}^{\mathbf{c}} \tilde{\mathcal{B}}^{2}$ let $L^{*}=\mathfrak{D}\left(L^{\dagger}\right)$. We say that $L^{*}$ is the dual of $L$. Note that $L \mapsto L^{*}$ is a contravariant functor $\mathcal{C}^{\mathbf{c}} \tilde{\mathcal{B}}^{2} \rightarrow \mathcal{C}^{\mathbf{c}} \tilde{\mathcal{B}}^{2}$ and that $L^{* *}=L$. We show how $L \mapsto L^{*}$ gives a rigid structure on $\mathcal{C}^{\mathbf{c}} \tilde{\mathcal{B}}^{2}$.

We have the following special case of 4.5 (a) (we use that $\mathbf{1}=\mathbf{1}^{\prime}$, see $4.7(\mathrm{~g}))$ :

$$
\operatorname{Hom}_{\mathcal{C}^{\mathbf{c}} \tilde{\mathcal{B}}^{2}}\left(\mathbf{1}, L \underline{\varrho} \mathfrak{D}\left(L^{\dagger}\right)\right)=\operatorname{Hom}_{\mathcal{C}^{\mathbf{c}} \tilde{\mathcal{B}}^{2}}(L, L)
$$

for any $L$ in $\mathcal{C}_{0}^{\mathbf{c}} \tilde{\mathcal{B}}^{2}$. Let $\xi_{L} \in \operatorname{Hom}_{\mathcal{C}^{\mathbf{c}} \tilde{\mathcal{B}}^{2}}\left(\mathbf{1}, L \circ \underline{D}\left(L^{\dagger}\right)\right)$ be the element corresponding under (a) to the identity homomorphism in $\operatorname{Hom}_{\mathcal{C}^{\mathbf{c}} \tilde{\mathcal{B}}^{2}}(L, L)$. Using (e) and 2.25(a) we have

$$
\begin{aligned}
\operatorname{Hom}_{\mathcal{C}^{\mathbf{c}} \tilde{\mathcal{B}}^{2}}\left(L \underline{\varrho} \mathfrak{D}\left(L^{\dagger}\right), \mathbf{1}\right) & =\operatorname{Hom}_{\mathcal{C}^{\mathbf{c}} \tilde{\mathcal{B}}^{2}}\left(\mathfrak{D}\left(\mathbf{1}^{\dagger}\right), \mathfrak{D}\left(\left(L \underline{\varrho} \mathfrak{D}\left(L^{\dagger}\right)\right)^{\dagger}\right)\right) \\
& =\operatorname{Hom}_{\mathcal{C}^{\mathbf{c}} \tilde{\mathcal{B}}^{2}}\left(\mathbf{1}, \mathfrak{D}\left(L^{\dagger}\right) \underline{\varrho} L\right) .
\end{aligned}
$$

Let $\xi_{L}^{\prime} \in \operatorname{Hom}_{\mathcal{C}^{\mathbf{c}} \tilde{\mathcal{B}}^{2}}\left(L \circ \mathfrak{D}\left(L^{\dagger}\right) \mathbf{1}\right)$ be the element corresponding under (b) to the element $\xi_{\mathfrak{D}\left(L^{\dagger}\right)} \in \operatorname{Hom}_{\mathcal{C}^{\mathbf{c}} \tilde{\mathcal{B}}^{2}}\left(\mathbf{1}, \mathfrak{D}\left(L^{\dagger}\right) \underline{\varrho} L\right)$. The elements $\xi_{L}, \xi_{L}^{\prime}$ define the rigid structure on $\mathcal{C}^{\mathbf{c}} \tilde{\mathcal{B}}^{2}$.

4.9. Let $\mathcal{Z}^{\mathbf{c}}$ be the centre (in the sense of Joyal and Street [6], Majid [26] and Drinfeld) of the monoidal abelian category $\mathcal{C}^{\mathbf{c}} \tilde{\mathcal{B}}^{2}$. By a general result on semisimple rigid monoidal categories in [4, Proposition 5.4], for any $L \in \mathcal{C}^{\mathbf{c}} \tilde{\mathcal{B}}^{2}$ one can define directly a central structure on the object

$$
I(L):=\oplus_{y \cdot \lambda \in \mathbf{c}} \mathbf{L}_{\lambda}^{\dot{y}} \underline{-} L \underline{-} \mathbf{L}_{y(\lambda)}^{\dot{y}^{-1}}
$$


of $\mathcal{C}^{\mathbf{c}} \tilde{\mathcal{B}}^{2}$ such that, denoting by $\overline{I(L)}$ the corresponding object of $\mathcal{Z}^{\mathbf{c}}$, we have canonically

$$
\operatorname{Hom}_{\mathcal{C}^{\mathbf{c}} \tilde{\mathcal{B}}^{2}}\left(L, L^{\prime}\right)=\operatorname{Hom}_{\mathcal{Z}^{\mathrm{c}}}\left(\overline{I(L)}, L^{\prime}\right)
$$

for any $L^{\prime} \in \mathcal{Z}^{\mathbf{c}}$. (We use that for $y \cdot \lambda \in \mathbf{c}$, the dual of the simple object $\mathbf{L}_{\lambda}^{\dot{y}}$ is $\mathbf{L}_{y(\lambda)}^{\dot{y}^{-1}}$.) The central structure on $I(L)$ can be described as follows: for any $X \in \mathcal{C}^{\mathbf{c}} \tilde{\mathcal{B}}^{2}$ we have canonically

$$
\begin{aligned}
& X \underline{\circ} I(L)=\oplus_{y \cdot \lambda \in \mathbf{c}} X_{\underline{\circ}} \mathbf{L}_{\lambda}^{\dot{y}} \underline{\circ} \underline{\varrho} \mathbf{L}_{y(\lambda)}^{\dot{y}^{-1}} \\
& =\oplus_{y \cdot \lambda \in \mathbf{c}, z \cdot \lambda^{\prime} \in \mathbf{c}} \operatorname{Hom}_{\mathcal{C}^{\mathbf{c}} \tilde{\mathcal{B}}^{2}}\left(\mathbf{L}_{\lambda^{\prime}}^{\dot{z}}, X \underline{\mathbf{L}_{\lambda}^{y}}\right) \otimes \mathbf{L}_{\lambda^{\prime}}^{\dot{z}} \underline{\dot{y}} L \underline{\mathbf{L}_{y}(\lambda)} \dot{y}^{-1} \\
& =\oplus_{y \cdot \lambda \in \mathbf{c}, z \cdot \lambda^{\prime} \in \mathbf{c}} \operatorname{Hom}_{\mathcal{C}^{\mathbf{c}} \tilde{\mathcal{B}}^{2}}\left(\mathbf{L}_{y(\lambda)}^{\dot{y}^{-1}}, \mathbf{L}_{z\left(\lambda^{\prime}\right)}^{\dot{z}^{-1}} \otimes X\right) \otimes \mathbf{L}_{\lambda^{\prime}}^{\dot{z}} \underline{\mathrm{o}} L \underline{\mathbf{L}_{y}} \dot{y}_{y(\lambda)}^{\dot{y}^{-1}} \\
& =\oplus_{z \cdot \lambda^{\prime} \in \mathbf{c}} \mathbf{L}_{\lambda^{\prime}}^{\dot{\dot{z}}} L \underline{\mathrm{O}} \mathbf{L}_{z\left(\lambda^{\prime}\right)}^{\dot{z}^{-1}} \otimes X=I(L) \underline{\circ} X .
\end{aligned}
$$

(The third equality uses 3.20(a).)

We show:

(b) If $z \cdot \lambda \in \mathbf{c}$ and $I\left(\mathbf{L}_{\lambda}^{\dot{z}}\right) \neq 0$ then $z \cdot \lambda \in \widetilde{W} \mathfrak{s}$.

For some $y \cdot \lambda^{\prime} \in \mathbf{c}$ we have $\mathbf{L}_{\lambda^{\prime}}^{\dot{y}} \underline{\mathbf{L}} \mathbf{L}_{\lambda}^{\dot{z}} \neq 0$ (hence $\lambda^{\prime}=z(l)$ ) and $\mathbf{L}_{\lambda}^{\dot{z}} \underline{\mathbf{L}^{\prime}} \dot{y}_{y\left(\lambda^{\prime}\right)}^{\dot{y}^{-1}} \neq 0$ (hence $\lambda=\lambda^{\prime}$ ). It follows that $z(\lambda)=\lambda$ and (b) is proved.

4.10. By $3.13(\mathrm{~d})$, for $z \cdot \lambda \in \breve{\mathbf{c}}$ we have canonically

$$
\underline{\mathfrak{b}}\left(\mathbb{L}_{\dot{\lambda}}^{\dot{z}}\right)=I\left(\mathbf{L}_{\lambda}^{\dot{z}}\right)
$$

as objects of $\mathcal{C}^{\mathbf{c}} \tilde{\mathcal{B}}^{2}$. Here $\underline{\mathfrak{b}}: \mathcal{C}_{0}^{\mathbf{c}} Z \rightarrow \mathcal{C}_{0}^{\mathbf{c}} \tilde{\mathcal{B}}^{2}$ in 3.13 is viewed as a functor $\underline{\mathfrak{b}}: \mathcal{C}^{\mathbf{c}} Z \rightarrow \mathcal{C}^{\mathbf{c}} \tilde{\mathcal{B}}^{2}$ as in 4.6. Now $I\left(\mathbf{L}_{\lambda}^{\dot{z}}\right)$ has a natural central structure (by 4.9) and $\underline{\mathfrak{b}}\left(\mathbb{L}_{\lambda}^{\dot{z}}\right)$ has a natural central structure (by 3.14(j)). By 3.21(b),

(b) these two central structures are compatible with the identification (a).

In view of (a), (b) we can reformulate 4.9(a) as follows.

Theorem 4.11. For any $z \cdot \lambda \in \breve{c}, L^{\prime} \in \mathcal{Z}^{\mathbf{c}}$ we have canonically

$$
\operatorname{Hom}_{\mathcal{C}^{\mathbf{c}} \tilde{\mathcal{B}}^{2}}\left(\mathbf{L}_{\lambda}^{\dot{z}}, L^{\prime}\right)=\operatorname{Hom}_{\mathcal{Z}}\left(\overline{\underline{\mathfrak{b}}\left(\mathbb{L}_{\lambda}^{\dot{z}}\right)}, L^{\prime}\right)
$$


where $\overline{\underline{\mathfrak{b}}(\mathbb{L}} \dot{\bar{z}})$ is $\underline{\mathfrak{b}}\left(\mathbb{L}_{\lambda}^{\dot{z}}\right)$ viewed as an object of $\mathcal{Z}^{\mathbf{c}}$ with the central structure given by $3.14(\mathrm{j})$.

4.12. We will state some variants (a)-(j) of results in 4.1-4.5 which will be needed in Section 6. Let $\delta_{0}: \mathcal{B} \rightarrow Z$ be map $B \mapsto\left(B, B, U_{B}\right)$. Let $\pi_{0}^{\prime}: \mathcal{B} \rightarrow \mathbf{p}$ be the obvious map.

(a) Let $\lambda \in \mathfrak{s}$ and let $w \in W_{\lambda}^{\prime}$ be such that $w \cdot \lambda \preceq \mathbf{c}$; let $i \in \mathbf{Z}$. If $i>a$ we have $\mathcal{H}^{i}\left(\delta_{0}^{*} \mathcal{L}_{\lambda}^{\dot{w} \sharp}[|w|]\right)=0$.

This can be deduced from 4.1(c) using that $e^{*} \mathcal{H}^{i}\left(\delta_{0}^{*} \mathcal{L}_{\lambda}^{\dot{w} \sharp}[|w|]\right)=\mathcal{H}^{i}\left(\delta^{*} L_{\lambda}^{\dot{w} \sharp}[|w|]\right)$ where $e: \tilde{\mathcal{B}} \rightarrow \mathcal{B}$ is the map $x \mathbf{U} \mapsto x \mathbf{B} x^{-1}$.

(b) If $L \in \mathcal{M} \preceq Z$ and $j>-a-\rho$ then $\left(\delta_{0}^{*} L\right)^{j}=0$.

We argue as in the proof of 4.1(d). We can assume that $L=\mathbb{L}_{\lambda}^{\dot{w}}$ with $w \cdot \lambda$ as in (a). It is enough to show that for any $k$ we have $\left(\mathcal{H}^{k}\left(\delta_{0}^{*} L\right)[-k]\right)^{j}=0$ that is $\left(\mathcal{H}^{k}\left(\delta_{0}^{*}\left(\mathcal{L}_{\lambda}^{\dot{w} \sharp}[|w|+\nu+\rho]\right)\right)[\nu]\right)^{j-k-\nu}=0$. Now $\mathcal{H}^{k}\left(\delta_{0}^{*}\left(\mathcal{L}_{\lambda}^{\dot{w} \sharp}[|w|+\nu+\rho]\right)\right)$ is a local system on $\mathcal{B}$ hence $\mathcal{H}^{k}\left(\delta_{0}^{*}\left(\mathcal{L}_{\lambda}^{\dot{w} \sharp}[|w|+\nu+\rho]\right)\right)[\nu]$ is a perverse sheaf on $\mathcal{B}$ so that we can assume that $j-k-\nu=0$. Thus it is enough to show that $\mathcal{H}^{j-\nu}\left(\delta_{0}^{*}\left(\mathcal{L}_{\lambda}^{\dot{w} \sharp}[|w|+\nu+\rho]\right)\right)=0$ or that $\mathcal{H}^{j+\rho}\left(\delta^{*}\left(L_{\lambda}^{\dot{w} \sharp}[|w|)\right)=0\right.$. This is indeed true by $(\mathrm{a})$.

(c) If $L \in \mathcal{M} \frac{\prec}{m} Z$ is pure of weight 0 and $j \in \mathbf{Z}$ then $\left(\delta_{0}^{*} L\right)^{j}$ is pure of weight $j$.

We argue as in the proof of 4.1(e). We can assume that $L=\mathbb{L}_{\lambda}^{\dot{w}}$ with $\lambda, w$ as in (a). It is enough to prove that for any $k,\left(\mathcal{H}^{k}\left(\delta_{0}^{*} L\right)[-k]\right)^{j}$ is pure of weight $j$ that is, $\left(\mathcal{H}^{k}\left(\delta_{0}^{*}\left(\mathcal{L}_{\lambda}^{\dot{w} \sharp}[|w|+\nu+\rho]\right)\right)[\nu]\right)^{j-k-\nu}((|w|+\nu+\rho) / 2)$ is pure of weight $j$. As in the proof of (b) we can assume that $j-k-\nu=0$. Thus it is enough to show that $\mathcal{H}^{j-\nu}\left(\delta_{0}^{*}\left(\mathcal{L}_{\lambda}^{\dot{w} \sharp}[|w|+\nu+\rho]\right)\right)[\nu]((|w|+\nu+\rho) / 2)$ is pure of weight $j$ or that $\mathcal{H}^{j+|w|+\rho}\left(\delta_{0}^{*}\left(\mathcal{L}_{\lambda}^{\dot{w} \sharp}\right)\right)\langle\nu\rangle((|w|+\rho) / 2)$ is pure of weight $j$. This follows from 4.1(b).

(d) Let $\lambda \in \mathfrak{s}, w \in W_{\lambda}^{\prime}$ be such that $w \cdot \lambda \in \mathbf{c}$. If $w \cdot \lambda \in \mathbf{D}_{\mathbf{c}}$ (see 1.12) then $\left(\delta_{0}^{*} \mathbb{L}_{\lambda}^{\dot{w}}\right)^{-a-\rho}=\beta_{w \cdot \lambda}\langle\nu\rangle((a+\rho) / 2)$ where $\beta_{w \cdot \lambda}$ is as in $4.1(\mathrm{f})$. If $w \cdot \lambda \notin \mathbf{D}_{\mathbf{c}}$ then $\left(\delta_{0}^{*} \mathbb{L}_{\lambda}^{\dot{w}}\right)^{-a-\rho}=0$. 
As in the proof of (c) we have

$$
\left(\delta_{0}^{*} \mathbb{L}_{\lambda}^{\dot{w}}\right)^{-a-\rho}=\mathcal{H}^{-a+|w|}\left(\delta_{0}^{*}\left(\mathcal{L}_{\lambda}^{\dot{w} \sharp}\right)\right)\langle\nu+\rho\rangle((|w|+\rho) / 2) .
$$

Setting $\beta_{w \cdot \lambda ; 0}=\mathcal{H}^{-a+|w|}\left(\delta_{0}^{*}\left(\mathcal{L}_{\lambda}^{\dot{w} \sharp}\right)\right)\langle\nu+\rho\rangle((-a+|w|) / 2)$ we have $\left(\delta_{0}^{*} \mathbb{L}_{\lambda}^{\dot{w}}\right)^{-a-\rho}$ $=\beta_{w \cdot \lambda ; 0}\langle\nu\rangle((a+\rho) / 2)$ where $\beta_{w \cdot \lambda ; 0}$ is a mixed vector space. If $e: \tilde{\mathcal{B}} \rightarrow \mathcal{B}$ is the obvious map, we have

$$
\begin{aligned}
\beta_{w \cdot \lambda ; 0} & =e^{*}\left(\mathcal{H}^{-a+|w|}\left(\delta_{0}^{*}\left(\mathcal{L}_{\lambda}^{\dot{w} \sharp}\right)\right)\langle\nu+\rho\rangle((-a+|w|) / 2)\right. \\
& =\mathcal{H}^{-a+|w|}\left(\delta^{*}\left(L_{\lambda}^{\dot{w} \sharp}\right)\right)\langle\nu+\rho\rangle((-a+|w|) / 2)=\beta_{w \cdot \lambda}
\end{aligned}
$$

where $\beta_{w \cdot \lambda}$ is as in 4.1(f). Hence the result follows from 4.1(f).

(e) Assume that $L \in \mathcal{M}_{m}(\mathcal{B})$ is $G$-equivariant so that $L=V \otimes \overline{\mathbf{Q}}_{l}\langle\nu\rangle$ where $V$ is a mixed vector space. If $j>\nu$ then $\left(\pi_{0 !}^{\prime} L\right)^{j}=0$.

We argue as in the proof of $4.2(\mathrm{a})$. We have $\mathcal{H}^{j}\left(p_{0 !}^{\prime} L\right)=V \otimes H_{c}^{j+\nu}\left(\tilde{\mathcal{B}}, \overline{\mathbf{Q}}_{l}\right)(\nu / 2)$. This is zero if $j+\nu>2 \nu$ since $\mathcal{B}$ is irreducible of dimension $\nu+\rho$.

(f) If $L \in \mathcal{M}_{\frac{\mathfrak{m}}{\mathrm{b}}} Z$ and $j>\nu-a-\rho$ then $\left(\pi_{0 !}^{\prime} \delta_{0}^{*} L\right)^{!}=0$. Moreover we have canonically $\left(\pi_{0 !}^{\prime} \delta_{0}^{*} L\right)^{\nu-a-\rho}=\left(\pi_{0 !}^{\prime}\left(\left(\delta_{0}^{*} L\right)^{-a-\rho}\right)\right)^{\nu}$.

The proof is almost identical to that of 4.2(b), using (b), (e) instead of 4.1(d), $4.2(\mathrm{a})$.

(g) Let $L \in \mathcal{C}_{0}^{\mathbf{c}} Z$. Then $\left(\pi_{0 !}^{\prime} \delta_{0}^{*} L\right)^{\nu-a-\rho}$ and $\left(\pi_{0 !}^{\prime}\left(\left(\delta_{0}^{*} L\right)^{-a-\rho}\right)\right)^{\nu}$ are pure of weight $\nu-a-\rho$.

We argue as in the proof of $4.2(\mathrm{f})$. We can assume that $L=\mathbb{L}_{\lambda}^{\dot{w}}$ where $w \cdot \lambda \in \breve{c}$. Using (d) we have

$$
\begin{aligned}
& \left(\pi_{0 !}^{\prime}\left(\left(\delta_{0}^{*} L\right)^{-a-\rho}\right)\right)^{\nu}((-a-\rho+\nu) / 2) \\
& =\left(\pi_{0 !}^{\prime}\left(\beta_{w \cdot \lambda}\langle\nu\rangle\right)((a+\rho) / 2)\right)^{\nu}((-a-\rho+\nu) / 2) \\
& =\beta_{w \cdot \lambda} \otimes\left(\pi_{0 !}^{\prime} \overline{\mathbf{Q}}_{l}\langle\nu\rangle\right)^{\nu}(\nu / 2)=\beta_{w \cdot \lambda} \otimes\left(\pi_{0 !}^{\prime} \overline{\mathbf{Q}}_{l}\right)^{2 \nu}(\nu)=\beta_{w \cdot \lambda} .
\end{aligned}
$$

Since $\beta_{w \cdot \lambda}$ is pure of weight 0 , we see that $\left(\pi_{0 !}^{\prime}\left(\left(\delta_{0}^{*} L\right)^{-a-\rho}\right)\right)^{\nu}$ is pure of weight $\nu-a-\rho$. Using (f) we deduce that $\left(\pi_{0 !}^{\prime} \delta_{0}^{*} L\right)^{\nu-a-\rho}$ is pure of weight $\nu-a-\rho$.

We set

$$
\mathbf{1}_{0}^{\prime}=\oplus_{d \cdot \lambda \in \mathbf{D}_{\mathbf{c}}} \beta_{d \cdot \lambda}^{*} \otimes \mathbb{L}_{\lambda}^{\dot{d}} \in \mathcal{C}_{0}^{\mathbf{c}} Z
$$


(h) For $L \in \mathcal{C}^{\mathbf{c}} Z$ we have canonically

$$
\operatorname{Hom}_{\mathcal{C}^{\mathrm{c}} Z}\left(\mathbf{1}_{0}^{\prime}, L\right)=\left(\pi_{0 !}^{\prime}\left(\left(\delta_{0}^{*} L\right)^{-a-\rho}\right)\right)^{\nu}((-a-\rho+\nu) / 2) .
$$

The proof is similar to that of 4.3(a); it uses (d) (instead of $4.1(\mathrm{f})$ ) and the proof of $(\mathrm{g})$.

For $L \in \mathcal{D}_{m}(Z)$ we set $L^{\dagger}=\mathfrak{h}^{*} L$ where $\mathfrak{h}: Z \rightarrow Z$ is as in 3.2.

(i) If $L \in \mathcal{C}_{0}^{\mathbf{c}} Z$ then $\mathfrak{D}\left(L^{\dagger}\right) \in \mathcal{C}_{0}^{\mathbf{c}} Z$.

This can be deduced from 4.4(b).

(j) For $L, L^{\prime}$ in $\mathcal{C}^{\mathbf{c}} Z$ we have canonically

$$
\operatorname{Hom}_{\mathcal{C}^{\mathrm{c}} Z}\left(\mathbf{1}_{0}^{\prime}, L \bullet L^{\prime}\right)=\operatorname{Hom}_{\mathcal{C}^{\mathrm{c}} Z}\left(\mathfrak{D}\left(L^{\prime \dagger}\right), L\right)
$$

This can be proved by the same method as 4.5 (b) or it can be deduced from 4.5(b) using the fully faithfulness of $\tilde{\epsilon}: \mathcal{C}_{0}^{\mathbf{c}} Z \rightarrow \mathcal{C}_{0}^{\mathbf{c}} \tilde{\mathcal{B}}^{2}$, the equality $\tilde{\epsilon} \mathbf{1}_{0}^{\prime}=\mathbf{1}$ and $3.22(\mathrm{a})$.

4.13. Let $\lambda \in \mathfrak{o}$. Using the decomposition 4.6 (c) of any object of $\mathcal{C}^{\mathbf{c}} \tilde{\mathcal{B}}^{2}$ we see that $\mathcal{C}^{\mathbf{c}} \tilde{\mathcal{B}}^{2}$ can be viewed as the category of "matrices" with entries in the abelian category $\mathcal{C}_{\lambda, \lambda}^{\mathbf{c}} \tilde{\mathcal{B}}^{2}$ (see 4.6). (This is a category version of the isomorphism $\Psi: \mathbf{H}_{\mathfrak{o}} \rightarrow \mathbf{E}$ in 1.11(v).) Using this and a result of Müger 27] it follows that $\mathcal{Z}_{\lambda}^{\mathbf{c}}$ is equivalent to the categorical centre of the abelian category $\mathcal{C}_{\lambda, \lambda}^{\mathbf{c}} \tilde{\mathcal{B}}^{2}$ with the monoidal structure induced by $\underline{\circ}($ see $4.6(\mathrm{~d}))$.

\section{Truncated Induction, Truncated Restriction, Truncated Convolution on $G$}

5.1. Let $\dot{Z}=\left\{\left(B, B^{\prime}, g\right) \in \mathcal{B} \times \mathcal{B} \times G ; g B g^{-1}=B^{\prime}\right\}$. We have a diagram

$$
Z \stackrel{f}{\leftarrow} \dot{Z} \stackrel{\pi}{\rightarrow} G
$$

where $f\left(B, B^{\prime}, g\right)=\left(B, B^{\prime}, g U_{B}\right), \pi\left(B, B^{\prime}, g\right)=g$. We define $\chi: \mathcal{D}(Z) \rightarrow$ $\mathcal{D}(G)$ and $\chi: \mathcal{D}_{m}(Z) \rightarrow \mathcal{D}_{m}(G)$ by

$$
\chi(L)=\pi_{!} f^{*} L
$$


For any $w \cdot \lambda \in W \mathfrak{s}$ we define $\mathfrak{R}_{\lambda}^{\dot{w}} \in \mathcal{D}_{m}(G), R_{\lambda}^{\dot{w}} \in \mathcal{D}_{m}(G)$ by

$$
\begin{aligned}
\mathfrak{R}_{\lambda}^{\dot{w}}=\chi\left(\mathcal{L}_{\lambda}^{\dot{w}}\right), R_{\lambda}^{\dot{w}}=\chi\left(\mathcal{L}_{\lambda}^{\dot{w} \sharp}\right) & \text { if } w \cdot \lambda \in \widetilde{W} \mathfrak{s}, \\
\mathfrak{R}_{\lambda}^{\dot{w}}=0, R_{\lambda}^{\dot{w}}=0 & \text { if } w \cdot \lambda \notin W \mathfrak{W} .
\end{aligned}
$$

Here $\widetilde{W} \mathfrak{s}$ is as in 3.3 .

We say that a simple perverse sheaf $A$ on $G$ is a character sheaf if the following equivalent conditions are satisfied:

- there exists $w \cdot \lambda \in W \mathfrak{s}$ and $j \in \mathbf{Z}$ such that $\left(A:\left(\Re_{\lambda}^{\dot{w}}\right)^{j}\right) \neq 0$;

- there exists $w \cdot \lambda \in W \mathfrak{s}$ and $j \in \mathbf{Z}$ such that $\left(A:\left(R_{\lambda}^{\dot{w}}\right)^{j}\right) \neq 0$.

(For the equivalence of these two conditions see [16, 12.7].) A character sheaf $A$ determines a $W$-orbit on $\mathfrak{s}$ : the set of $\lambda \in \mathfrak{s}$ such that $\left(A:\left(\mathfrak{R}_{\lambda}^{\dot{w}}\right)^{j}\right) \neq 0$ for some $w \in W$ and some $j$ (or equivalently $\left(A:\left(R_{\lambda}^{\dot{w}}\right)^{j}\right) \neq 0$ for some $w \in W$ and some $j$ ), see [16, 11.2(a), 12.7]. We say that $A$ is an o-character sheaf if the $W$-orbit on $\mathfrak{s}$ determined by $A$ is $\mathfrak{o}$ (as in 2.14). Let $C S_{\mathfrak{o}}$ be a set of representatives for the isomorphism classes of $\mathfrak{o}$-character sheaves on $G$.

By [16, 14.11], for any $\lambda \in \mathfrak{o}$ there exists a pairing $C S_{\mathfrak{o}} \times \operatorname{Irr} W_{\lambda}^{\prime} \rightarrow \overline{\mathbf{Q}}_{l}$, $(A, e) \mapsto b_{A, e}$ such that for any $A \in C S_{\mathfrak{o}}$, any $z \in W_{\lambda}^{\prime}$ and any $j \in \mathbf{Z}$ we have

$$
\left(A:\left(R_{\lambda}^{\dot{z}}\right)^{j}\right)=\left(j-\Delta-|z| ;(-1)^{j+\Delta} \sum_{e \in \operatorname{Irr}\left(W_{\lambda}^{\prime}\right)} b_{A, e} \operatorname{tr}\left(c_{z \cdot \lambda}, e^{v}\right)\right) .
$$

Here $e^{v}$ is as in 1.12. (When $z \cdot \lambda \notin W \mathfrak{W}$, both sides are zero.) By the results in 1.12 this can be reformulated as follows.

There exists a pairing $C S_{\mathfrak{o}} \times \operatorname{Irr}_{\mathfrak{o}}\left(W \mathbf{T}_{n}\right) \rightarrow \overline{\mathbf{Q}}_{l},(A, E) \mapsto b_{A, E}$ such that for any $A \in C S_{\mathfrak{o}}$, any $\lambda \in \mathfrak{o}$, any $z \in W$ and any $j \in \mathbf{Z}$ we have

(a) $\quad\left(A:\left(R_{\lambda}^{\dot{z}}\right)^{j}\right)=(-1)^{j+\Delta}\left(j-\Delta-|z| ; \sum_{E \in \operatorname{Irr}_{\mathfrak{o}}\left(W \mathbf{T}_{n}\right)} b_{A, E} \operatorname{tr}\left(c_{z \cdot \lambda}, E^{v}\right)\right)$

where $E^{v}$ is as in 1.12 ; if $E^{\prime}$ is an $\overline{\mathbf{Q}}_{l}\left[W \mathbf{T}_{n}\right]$-module isomorphic to $\oplus_{E \in \operatorname{Irr}\left(W \mathbf{T}_{n}\right)} E^{\oplus m_{E}}$ (with $m_{E} \in \mathbf{N}$ ) we set

$$
b_{A, E^{\prime}}=\sum_{E \in \operatorname{Irr}_{\mathfrak{o}}\left(W \mathbf{T}_{n}\right)} m_{E} b_{A, E} .
$$


In particular if $E^{\prime} \in \operatorname{Irr}\left(W \mathbf{T}_{n}\right)-\operatorname{Irr}_{\mathfrak{o}}\left(W \mathbf{T}_{n}\right)$, we have $b_{A, E^{\prime}}=0$. Moreover, given $A \in C S_{\mathfrak{o}}$, there is a unique two-sided cell $\mathbf{c}_{A}$ of $W \mathfrak{s}$ such that $b_{A, E}=0$ whenever $E \in \operatorname{Irr}_{\mathfrak{o}}\left(W \mathbf{T}_{n}\right)$ satisfies $\mathbf{c}_{E} \neq \mathbf{c}_{A}$. (This follows from 16, 16.7].) We have necessarily $\mathbf{c}_{A} \subset\{w \cdot \lambda \in W \mathfrak{s} ; \lambda \in \mathfrak{o}\}$. As in [21, 41.8], [22, 44.18], we see that:

(b) $\left(A:\left(R_{\lambda}^{\dot{z}}\right)^{j}\right) \neq 0$ for some $z \cdot \lambda \in \mathbf{c}_{A}, j \in \mathbf{Z}$; conversely, if $\left(A:\left(R_{\lambda}^{\dot{z}}\right)^{j}\right) \neq 0$ for $z \cdot \lambda \in W \mathfrak{s}, j \in \mathbf{Z}$, then $\mathbf{c}_{A} \preceq z \cdot \lambda$.

Let $a_{A}$ be the value of the $a$-function on $\mathbf{c}_{A}$. If $z \cdot \lambda \in W \mathfrak{s}, E \in \operatorname{Irr}_{\mathfrak{o}}\left(W \mathbf{T}_{n}\right)$ satisfy $\operatorname{tr}\left(c_{z \cdot \lambda}, E^{v}\right) \neq 0$ then $\mathbf{c}_{E} \preceq z \cdot \lambda$; if in addition we have $z \cdot \lambda \in \mathbf{c}_{E}$ then from the definitions we have

$$
\operatorname{tr}\left(c_{z \cdot \lambda}, E^{v}\right)=\sum_{h \geq 0} \gamma_{z \cdot \lambda, E, h} v^{a_{E}-h}
$$

where $\gamma_{z \cdot \lambda, E, h} \in \overline{\mathbf{Q}}_{l}$ is zero for large $h, \gamma_{z \cdot \lambda, E, 0}=\operatorname{tr}\left(t_{z, \lambda}, E^{\infty}\right)$ and $a_{E}$ is as in 1.13. Hence from (a) we see that for $A \in C S_{\mathfrak{o}}$ and $\lambda \in \mathfrak{o}, z \in W, j \in \mathbf{Z}$, the following holds:

(c) $\left(A:\left(R_{\lambda}^{\dot{z}}\right)^{j}\right)=0$ unless $\mathbf{c}_{A} \preceq z \cdot \lambda$; if $z \cdot \lambda \in \mathbf{c}_{A}$ then

$$
\left(A:\left(R_{\lambda}^{\dot{z}}\right)^{j}\right)=(-1)^{j+\Delta}\left(j-\Delta-|z| ; \sum_{E \in \operatorname{Irr}_{\mathfrak{o}}\left(W \mathbf{T}_{n}\right) ; \mathbf{c}_{E}=\mathbf{c}_{A} ; h \geq 0} b_{A, E} \gamma_{z \cdot \lambda, E, h} v^{a_{A}-h}\right)
$$

which is 0 unless $j-\Delta-|z| \leq a_{A}$.

Recall that c, $a$ are as in 2.14 .

Let $\mathcal{M} \preceq G$ (resp. $\mathcal{M}^{\prec} G$ ) be the category of perverse sheaves on $G$ whose composition factors are all of the form $A \in C S_{\mathfrak{o}}$ with $\mathbf{c}_{A} \preceq \mathbf{c}$ (resp. $\mathbf{c}_{A} \prec$ c). Let $\mathcal{D}^{\preceq} G$ (resp. $\mathcal{D}^{\prec} G$ ) be the subcategory of $\mathcal{D}(G)$ whose objects are complexes $K$ such that $K^{j}$ is in $\mathcal{M}^{\prec} G$ (resp. $\mathcal{M}^{\prec} G$ ) for any $j$. Let $\mathcal{D}_{\bar{m}}^{\preceq} G$ (resp. $\mathcal{D}_{m}^{\prec} G$ ) be the subcategory of $\mathcal{D}_{m}(G)$ whose objects are also in $\mathcal{D}^{\preceq} G$ (resp. $\mathcal{D}^{\prec} G$ ).

Let $\lambda \in \mathfrak{o}, z \in W$. From (c) we deduce:

(d) If $z \cdot \lambda \preceq$ c then $\left(R_{\lambda}^{\dot{z}}\right)^{j} \in \mathcal{M} \preceq G$ for all $j \in \mathbf{Z}$.

(e) If $z \cdot \lambda \in \mathbf{c}$ and $j>a+\Delta+|z|$ then $\left(R_{\lambda}^{\dot{z}}\right)^{j} \in \mathcal{M}^{\prec} G$.

(f) If $z \cdot \lambda \prec \mathbf{c}$ then $\left(R_{\lambda}^{\dot{z}}\right)^{j} \in \mathcal{M}^{\prec} G$ for all $j \in \mathbf{Z}$. 
5.2. Let $C S_{\mathbf{c}}=\left\{A \in C S_{\mathfrak{o}} ; \mathbf{c}_{A}=\mathbf{c}\right\}$. For any $z \cdot \lambda \in W \mathfrak{s}$ we set

$$
n_{z}=a(z)+\Delta+|z|
$$

Let $A \in C S_{\mathbf{c}}$ and let $z \cdot \lambda \in \mathbf{c}$. We have

$$
\left(A:\left(R_{\lambda}^{\dot{z}}\right)^{n_{z}}\right)=(-1)^{a+|z|} \sum_{E \in \operatorname{Irr}\left(W \mathbf{T}_{n}\right) ; \mathbf{c}_{E}=\mathbf{c}} b_{A, E} \operatorname{tr}\left(t_{z \cdot \lambda}, E^{\infty}\right) .
$$

Indeed, from 5.1(a) we have

$$
\left(A:\left(R_{\lambda}^{\dot{z}}\right)^{n_{z}}\right)=(-1)^{a+|z|} \sum_{E \in \operatorname{Irr}\left(W \mathbf{T}_{n}\right) ; \mathbf{c}_{E}=\mathbf{c}} b_{A, E}\left(a ; \operatorname{tr}\left(c_{z \cdot \lambda}, E^{v}\right)\right)
$$

and it remains to use that $\left(a ; \operatorname{tr}\left(c_{z \cdot \lambda}, E^{v}\right)\right)=\operatorname{tr}\left(t_{z \cdot \lambda}, E^{\infty}\right)$. We show:

(b) For any $A \in C S_{\mathbf{c}}$ there exists $E \in \operatorname{Irr}\left(W \mathbf{T}_{n}\right)$ such that $\mathbf{c}_{E}=\mathbf{c}, b_{A, E} \neq 0$.

Assume that this is not so. Then, using 5.1(a), for any $\lambda \in \mathfrak{o}$, any $z \in W$ and any $j \in \mathbf{Z}$ we have $\left(A:\left(R_{\lambda}^{\dot{z}}\right)^{j}\right)=0$. This contradicts the assumption that $A \in C S_{\mathfrak{o}}$.

(c) For any $A \in C S_{\mathbf{c}}$ there exists $z \cdot \lambda \in \mathbf{c}$ such that $\left(A:\left(R_{\lambda}^{\dot{z}}\right)^{n_{z}}\right) \neq 0$.

Assume that this is not so. Then, using (a), we see that

$$
\sum_{E \in \operatorname{Irr}\left(W \mathbf{T}_{n}\right) ; \mathbf{c}_{E}=\mathbf{c}} b_{A, E} \operatorname{tr}\left(t_{z \cdot \lambda}, E^{\infty}\right)=0
$$

for any $z \cdot \lambda \in \mathbf{c}$. Using this and (b) we see that the linear functions $t_{z \cdot \lambda} \mapsto$ $\operatorname{tr}\left(t_{z \cdot \lambda}, E^{\infty}\right)$ on $\mathbf{J}_{\mathbf{c}}$ (for various $E \in \operatorname{Irr}\left(W \mathbf{T}_{n}\right)$ such that $\mathbf{c}_{E}=\mathbf{c}$ ) are linearly dependent. This is a contradiction since the $E^{\infty}$ form a complete set of simple modules for the semisimple algebra $\mathbf{J}_{\mathbf{c}}$.

We show:

(d) Let $z \cdot \lambda \in \mathbf{c}$ be such that $\left(R_{\lambda}^{\dot{z}}\right)^{n_{z}} \neq 0$. Then $z \cdot \lambda \underset{\text { left }}{\sim} z^{-1} \cdot z(\lambda)$. In particular we have $z \in W_{\lambda}^{\prime}$ and $z, z^{-1}$ are in the same left cell of $W_{\lambda}^{\prime}$.

Using (a) we see that there exists $E \in \operatorname{Irr}\left(W \mathbf{T}_{n}\right)$ such that $\operatorname{tr}\left(t_{z \cdot \lambda}, E^{\infty}\right) \neq 0$. We have $E^{\infty}=\oplus_{d \cdot \lambda_{1} \in \mathbf{D}} t_{d \cdot \lambda_{1}} E^{\infty}$ and $t_{z \cdot \lambda}: E^{\infty} \rightarrow E^{\infty}$ maps the summand $t_{d \cdot \lambda_{1}} E^{\infty}$ where $z \cdot \lambda \underset{\text { left }}{\sim} d \cdot \lambda_{1}$ into $t_{d^{\prime} \cdot \lambda_{1}^{\prime}} E^{\infty}$ where $d \cdot \lambda_{1}^{\prime} \in \mathbf{D}, d^{\prime} \cdot \lambda_{1}^{\prime} \underset{\text { left }}{\sim} z^{-1} \cdot z(\lambda)$ and 
all other summands to 0 . Since $\operatorname{tr}\left(t_{z \cdot \lambda}, E^{\infty}\right) \neq 0$, we must have $t_{d \cdot \lambda_{1}} E^{\infty}=$ $t_{d^{\prime} \cdot \lambda_{1}^{\prime}} E^{\infty} \neq 0$ hence $d \cdot \lambda_{1}=d^{\prime} \cdot \lambda_{1}^{\prime}$ and $z \cdot \lambda \underset{\text { left }}{\sim} z^{-1} \cdot z(\lambda)$. This proves $(\mathrm{d})$.

5.3. We show:

(a) If $L \in \mathcal{D}^{\preceq} Z$ then $\chi(L) \in \mathcal{D}^{\preceq} G$. If $L \in \mathcal{D}^{\prec} Z$ then $\chi(L) \in \mathcal{D}^{\prec} G$.

(b) If $L \in \mathcal{M} Z$ and $j>a+\nu$ then $(\chi(L))^{j} \in \mathcal{M}^{\prec} G$.

It is enough to prove (a), (b) assuming in addition that $L=\mathbb{L}_{\lambda}^{\dot{z}}$ where $z \cdot \lambda \in \widetilde{W} \mathfrak{s}, z \cdot \lambda \preceq \mathbf{c}$. Then (a) follows from 5.1(d), (f). In the setup of (b) we have

$$
\left(\chi\left(\mathbb{L}_{\lambda}^{\dot{z}}\right)\right)^{j}=\left(R_{\lambda}^{\dot{z}}\right)^{j+|z|+\nu+\rho}((|z|+\nu+\rho) / 2)
$$

and this is in $\mathcal{M}^{\prec} G$ since $j+|z|+\nu+\rho>a+\Delta+|z|$, see 5.1(e).

5.4. Let $\mathcal{C} G$ be the subcategory of $\mathcal{M}(G)$ consisting of semisimple objects. Let $\mathcal{C}_{0} G$ be the subcategory of $\mathcal{M}_{m}(G)$ consisting of those $K$ such that $K$ is pure of weight zero. Let $\mathcal{C}^{\mathbf{c}} G$ be the subcategory of $\mathcal{M}(G)$ consisting of objects which are direct sums of objects of the form $A \in C S_{\mathbf{c}}$. Let $\mathcal{C}_{0}^{\mathbf{c}} G$ be the subcategory of $\mathcal{C}_{0}^{\text {a }} G$ consisting of those $K$ such that, as an object of $\mathcal{C}^{\mathbf{a}} G, K$ belongs to $\mathcal{C}^{\mathbf{c}} G$. For $K \in \mathcal{C}_{0}^{\mathbf{A}} G$ let $\underline{K}$ be the largest subobject of $K$ such that as an object of $\mathcal{C}^{\mathbf{M}} G$, we have $\underline{K} \in \mathcal{C}^{\mathbf{c}} G$.

5.5. For $L \in \mathcal{C}_{0}^{\mathbf{c}} Z$ we set

$$
\underline{\chi}(L)=\underline{(\chi(L))^{a+\nu}}((a+\nu) / 2)=\underline{(\chi(L))^{\{a+\nu\}}} \in \mathcal{C}_{0}^{\mathbf{c}} G .
$$

(The last equality uses that $\pi$ in 5.1 is proper hence it preserves purity.) The functor $\underline{\chi}: \mathcal{C}_{0}^{\mathbf{c}} Z \rightarrow \mathcal{C}_{0}^{\mathbf{c}} G$ is called truncated induction. For $z \cdot \lambda \in \breve{\mathbf{c}}$ we have

$$
\underline{\chi}\left(\mathbb{L}_{\lambda}^{\dot{z}}\right)=\underline{\left(R_{\lambda}^{\dot{z}}\right)^{n_{z}}}\left(n_{z} / 2\right)
$$

Indeed,

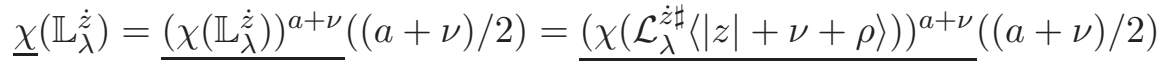

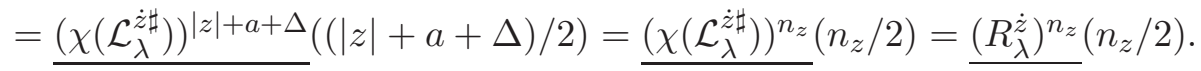

Using (a) and 5.2(d) we see that: 
(d) If $z \cdot \lambda \in \breve{\mathbf{c}}$ is such that $\underline{\chi}\left(\mathbb{L}_{\lambda}^{\dot{\dot{z}}}\right) \neq 0$ then $z \cdot \lambda \underset{\text { left }}{\sim} z^{-1} \cdot z(\lambda)$. In particular we have $z \in W_{\lambda}^{\prime}$ and $z, z^{-1}$ are in the same left cell of $W_{\lambda}^{\prime}$.

5.6. As in 1.9 we shall denote by $\tau: \mathbf{H}^{\infty} \rightarrow \mathbf{Z}$ the group homomorphism such that $\tau\left(t_{z \cdot \lambda}\right)=1$ if $z \cdot \lambda \in \mathbf{D}$ and $\tau\left(t_{z \cdot \lambda}\right)=0$ if $z \cdot \lambda \in W \mathfrak{s}-\mathbf{D}$. For $z \cdot \lambda, z^{\prime} \cdot \lambda^{\prime}$ in $\mathbf{c}$ we show:

(a) $\operatorname{dim} \operatorname{Hom}_{\mathcal{C}^{\mathbf{c}} G}\left(\underline{\chi}\left(\mathbb{L}_{\lambda}^{\dot{z}}\right), \underline{\chi}\left(\mathbb{L}_{\lambda^{\prime}}^{\dot{z}^{\prime}}\right)\right)=\sum_{y \in W ; y \cdot \lambda^{\prime} \in \mathbf{c}} \tau\left(t_{y^{-1} \cdot y\left(\lambda^{\prime}\right)} t_{z \cdot \lambda} t_{y \cdot \lambda^{\prime}} t_{z^{\prime-1} \cdot \lambda^{\prime}}\right)$.

Using 5.5(a) and the definitions we see that the left hand side of (a) equals

$$
\sum_{A \in C S_{\mathbf{c}}}\left(A:\left(R_{\lambda}^{\dot{z}}\right)^{n_{z}}\right)\left(A:\left(R_{\lambda^{\prime}}^{\dot{z}^{\prime}}\right)^{n_{z^{\prime}}}\right)
$$

Using 5.2(a) and the analogous identity for $\left(A:\left(R_{\lambda^{\prime}}^{\dot{z}^{\prime}}\right)^{n} z_{z^{\prime}}\right)$ in which the field automorphism () : $\overline{\mathbf{Q}}_{l} \rightarrow \overline{\mathbf{Q}}_{l}$ (see 1.16) is applied to both sides (the left hand side is fixed by ()$\left.^{\mathbf{N}}\right)$, we see that the left hand side of (a) equals

$$
\begin{gathered}
(-1)^{|z|+\left|z^{\prime}\right|} \sum_{E, E^{\prime} \in \operatorname{Irr}\left(W \mathbf{T}_{n}\right) ; \mathbf{c}_{E}=\mathbf{c}_{E^{\prime}}=\mathbf{c}} \\
\sum_{A \in C S_{\mathbf{c}}} b_{A, E} b_{A, E^{\prime}}^{\boldsymbol{\phi}} \operatorname{tr}\left(t_{z \cdot \lambda}, E^{\infty}\right)\left(\operatorname{tr}\left(t_{z^{\prime} \cdot \lambda^{\prime}}, E^{\prime \infty}\right)\right)
\end{gathered}
$$

Replacing in the last sum $\sum_{A \in C S_{\mathbf{c}}} b_{A, E} b_{A, E^{\prime}}^{\mathbf{\phi}}$ by 1 if $E^{\prime}=E$ and by 0 if $E^{\prime} \neq E($ see $[20,35.18(\mathrm{~g})])$ we see that the left hand side of (a) equals

$$
(-1)^{|z|+\left|z^{\prime}\right|} \sum_{E \in \operatorname{Irr}\left(W \mathbf{T}_{n}\right) ; \mathbf{c}_{E}=\mathbf{c}} \operatorname{tr}\left(t_{z \cdot \lambda}, E^{\infty}\right)\left(\operatorname{tr}\left(t_{z^{\prime} \cdot \lambda^{\prime}}, E^{\infty}\right)\right)^{\mathbf{\omega}}
$$

By [20, 34.17], for $E \in \operatorname{Irr}\left(W \mathbf{T}_{n}\right)$ and $h \in \mathbf{H}$ we have $\operatorname{tr}\left(h^{b}, E^{v}\right)=\operatorname{tr}\left(h, E^{v}\right)$. where ()$^{\mathbf{\top}}: \overline{\mathbf{Q}}_{l}(v) \rightarrow \overline{\mathbf{Q}}_{l}(v)$ is as in 1.16; in particular, for $w \cdot \lambda \in W \mathfrak{s}$ we have $\operatorname{tr}\left(c_{w \cdot \lambda}, E^{v}\right)=\operatorname{tr}\left(c_{w^{-1} \cdot w(\lambda)}, E^{v}\right)$. Taking the coefficient of $v^{a}$ in the two sides of the last equality we deduce $\operatorname{tr}\left(t_{w \cdot \lambda}, E^{\infty}\right)=\operatorname{tr}\left(t_{w^{-1} \cdot w(\lambda)}, E^{\infty}\right)$. Thus the left hand side of (a) equals

$$
(-1)^{|z|+\left|z^{\prime}\right|} \sum_{E \in \operatorname{Irr}\left(W \mathbf{T}_{n}\right), \mathbf{c}_{E}=\mathbf{c}} \operatorname{tr}\left(t_{z \cdot \lambda}, E^{\infty}\right) \operatorname{tr}\left(t_{z^{\prime-1} \cdot \lambda^{\prime}}, E^{\infty}\right) .
$$

(Recall that $z^{\prime}\left(\lambda^{\prime}\right)=z^{\prime}$.) This is equal to $(-1)^{|z|+\left|z^{\prime}\right|}$ times the trace of the linear map $\xi \mapsto t_{z \cdot \lambda} \xi t_{z^{\prime-1} \cdot \lambda^{\prime}}$ on $\mathbf{J}_{\mathbf{c}}$; hence it is equal to the sum over $y \cdot \lambda_{1} \in \mathbf{c}$ 
of the coefficient of $t_{y \cdot \lambda_{1}}$ in $t_{z \cdot \lambda} t_{y \cdot \lambda_{1}} t_{z^{\prime-1} \cdot \lambda^{\prime}}$; this coefficient is 0 if $\lambda_{1} \neq \lambda^{\prime}$ while if $\lambda_{1}=\lambda^{\prime}$ it is equal to

$$
\tau\left(t_{y^{-1} \cdot y\left(\lambda^{\prime}\right)} t_{z \cdot \lambda} t_{y \cdot \lambda^{\prime}} t_{z^{\prime-1} \cdot \lambda^{\prime}}\right)
$$

(We use 1.9(a) for $\mathbf{H}^{\infty}$.) Thus we have

$$
\operatorname{dim} \operatorname{Hom}_{\mathcal{C}^{\mathbf{c}} G}\left(\underline{\chi}\left(\mathbb{L}_{\lambda}^{\dot{z}}\right), \underline{\chi}\left(\mathbb{L}_{\lambda^{\prime}}^{\dot{z}^{\prime}}\right)\right)=(-1)^{|z|+\left|z^{\prime}\right|} \sum_{y \in W ; y \cdot \lambda^{\prime} \in \mathbf{c}} \tau\left(t_{y^{-1} \cdot y\left(\lambda^{\prime}\right)} t_{z \cdot \lambda} t_{y \cdot \lambda^{\prime}} t_{z^{\prime-1} \cdot \lambda^{\prime}}\right) .
$$

Since $\operatorname{dim} \operatorname{Hom}_{\mathcal{C}^{\mathrm{c}} G}\left(\underline{\chi}\left(\mathbb{L}_{\lambda}^{\dot{z}}\right), \underline{\chi}\left(\mathbb{L}_{\lambda^{\prime}}^{\dot{z}^{\prime}}\right)\right) \in \mathbf{N}$ and the last sum is in $\mathbf{N}$, it follows that (a) holds.

The proof above shows also that $\operatorname{dim} \operatorname{Hom}_{\mathcal{C}^{\mathrm{c}} G}\left(\underline{\chi}\left(\mathbb{L}_{\lambda}^{\dot{z}}\right), \underline{\chi}\left(\mathbb{L}_{\dot{\lambda}^{\prime}}^{\dot{z}^{\prime}}\right)\right)=0$ whenever $(-1)^{|z|+\left|z^{\prime}\right|}=-1$.

5.7. Let $L \in \mathcal{C}_{0}^{\mathbf{c}} Z$. We show that $\mathfrak{D}(L) \in \mathcal{C}_{0}^{\tilde{\mathbf{c}}} Z$. It is enough to note that for $w \cdot \lambda \in \breve{\mathbf{c}}$ and $\omega \in \kappa_{q}^{-1}(w)$ we have

(a) $\mathfrak{D}\left(\mathbb{L}_{\lambda}^{\omega}\right)=\mathbb{L}_{\lambda^{-1}}^{\omega}$

We show:

(b) We have canonically $\underline{\chi}(\mathfrak{D}(L))=\mathfrak{D}(\underline{\chi}(L))$ where the first $\underline{\chi}$ is relative to $\tilde{\mathbf{c}}$ instead of $\mathbf{c}$.

By the relative hard Lefschetz theorem [1, 5.4.10] applied to the projective morphism $\pi$ (see 5.1) and to $f^{*} L\langle\nu\rangle$ (a perverse sheaf of pure weight 0 on $\dot{Z}$, see 5.1$)$ we have canonically for any $i$ :

$$
\left(\pi ! f^{*} L\langle\nu\rangle\right)^{-i}=\left(\pi ! f^{*} L\langle\nu\rangle\right)^{i}(i)
$$

We have used the fact that $f$ is smooth with fibres of dimension $\nu$. This also shows that

$$
\mathfrak{D}(\chi(\mathfrak{D}(L)))=\chi(L)\langle 2 \nu\rangle \text {. }
$$

Using (d) we have

$$
\left.\mathfrak{D}(\underline{\chi}(\mathfrak{D}(L)))=\mathfrak{D}\left((\chi(\mathfrak{D}(L)))^{a+\nu}((a+\nu) / 2)\right)\right)
$$




$$
\begin{aligned}
& =(\mathfrak{D}(\chi(\mathfrak{D}(L))))^{-a-\nu}((-a-\nu) / 2) \\
& =(\chi(L)\langle 2 \nu\rangle)^{-a-\nu}((-a-\nu) / 2)=(\chi(L)\langle\nu\rangle)^{-a}(-a / 2) .
\end{aligned}
$$

Hence using (c) we have

$$
\mathfrak{D}(\underline{\chi}(\mathfrak{D}(L)))=(\chi(L)\langle\nu\rangle)^{a}(a / 2)=(\chi(L))^{a+\nu}((a+\nu) / 2)=\underline{\chi}(L) .
$$

This proves (b).

5.8. Let $z \cdot \lambda \in \mathbf{D}_{\mathbf{c}}$ and let $\Lambda_{z \cdot \lambda}$ be the left cell of $W \mathfrak{s}$ containing $z \cdot \lambda$. We show:

(a) $\left(A: \underline{\chi}\left(\mathbb{L}_{\lambda}^{\dot{z}}\right)\right)=b_{A,\left[\Lambda_{z, \lambda}\right]}$ for any $A \in C S_{\mathbf{c}}$.

Using 1.12(a) we see that for any $E \in \operatorname{Irr}\left(W \mathbf{T}_{n}\right), \operatorname{tr}\left(t_{z \cdot \lambda}, E^{\infty}\right)$ is equal to the multiplicity of $E$ in the $\overline{\mathbf{Q}}_{l}\left[W \mathbf{T}_{n}\right]$-module $\left[\Lambda_{z \cdot \lambda}\right]$. Hence, using 5.5(a) and $5.2(\mathrm{a})$, we have

$$
\begin{aligned}
\left(A: \underline{\chi}\left(\mathbb{L}_{\lambda}^{\dot{z}}\right)\right) & =(-1)^{a+|z|} \sum_{E \in \operatorname{Irr}\left(W \mathbf{T}_{n}\right) ; \mathbf{c}_{E}=\mathbf{c}} b_{A, E}\left(\text { multiplicity of } E \text { in }\left[\Lambda_{\delta}\right]\right) \\
& =(-1)^{a+|z|} b_{A,\left[\Lambda_{z, \lambda}\right]} .
\end{aligned}
$$

It is enough to show that $a+|z|=0 \bmod 2$. Since $z$ is a distinguished involution of $W_{\lambda}$, the coefficient of $v^{-a}$ in $p_{1, z}^{\lambda}$ (see 1.8) is nonzero (see [18, 14.1]). Using now [18, 5.4(b)] we deduce that $|z|_{\lambda}=a \bmod 2$. It remains to note that $|z|_{\lambda}=|z| \bmod 2$. (Indeed, $W_{\lambda}$ is generated by elements $u \in W_{\lambda}$ such that $|u|_{\lambda}=1$ and such $u$ are reflections in $W$ so that $|u|$ is odd.)

5.9. We define $\zeta: \mathcal{D}(G) \rightarrow \mathcal{D}(Z)$ and $\zeta: \mathcal{D}_{m}(G) \rightarrow \mathcal{D}_{m}(Z)$ by $\zeta(K)=f_{!} \pi^{*} K$ where $Z \stackrel{f}{\leftarrow} \dot{Z} \stackrel{\pi}{\rightarrow} G$ is as in $5.1($ a). We show:

(a) For any $L \in \mathcal{D}(Z)$ or $L \in \mathcal{D}_{m}(Z)$ we have $\mathfrak{b}^{\prime \prime}(L)=\zeta(\chi(L))$.

We have $\zeta(\chi(L))=f_{!} \pi^{*} \pi_{!} f^{*}(L)$. We have

$$
\dot{Z} \times{ }_{G} \dot{Z}=\left\{\left(\left(B_{0}, B_{1}, B_{2}, B_{3}\right), g\right) \in \mathcal{B}^{4} \times G ; g B_{0} g^{-1}=B_{3}, g B_{1} g^{-1}=B_{2}\right\} .
$$

We have a cartesian diagram 


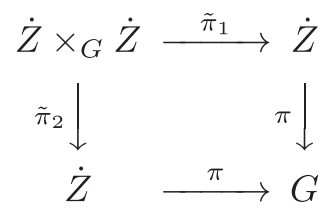

where $\tilde{\pi}_{1}\left(\left(B_{0}, B_{1}, B_{2}, B_{3}\right), g\right)=\left(B_{0}, B_{3}, g\right), \tilde{\pi}_{2}\left(\left(B_{0}, B_{1}, B_{2}, B_{3}\right), g\right)=\left(B_{1}\right.$, $\left.B_{2}, g\right)$. It follows that $\pi^{*} \pi_{!}=\tilde{\pi}_{1 !} \tilde{\pi}_{2}^{*}$. Thus

$$
\zeta(\chi(L))=f_{!} \tilde{\pi}_{1 !} \tilde{\pi}_{2}^{*} f^{*}(L)=\left(f \tilde{\pi}_{1}\right) !\left(f \tilde{\pi}_{2}\right)^{*}(L) .
$$

Define $\pi_{1}^{\prime}: \dot{Z} \times_{G} \dot{Z} \rightarrow Z, \pi_{2}^{\prime}: \dot{Z} \times_{G} \dot{Z} \rightarrow Z$ by

$$
\begin{aligned}
& \pi_{1}^{\prime}\left(\left(B_{0}, B_{1}, B_{2}, B_{3}\right), g\right)=\left(B_{0}, B_{3}, g U_{B_{0}}\right), \\
& \pi_{2}^{\prime}\left(\left(B_{0}, B_{1}, B_{2}, B_{3}\right), g\right)=\left(B_{1}, B_{2}, g U_{B_{1}}\right) .
\end{aligned}
$$

Then $\pi_{1}^{\prime}=f \tilde{\pi}_{1}, \pi_{2}^{\prime}=f \tilde{\pi}_{2}$ and $\zeta(\chi(L))=\pi_{1 !}^{\prime} \pi_{2}^{\prime *}(L)$. We have an isomorphism $\diamond \mathcal{Y} \rightarrow \dot{Z} \times{ }_{G} \dot{Z}$ induced by

$$
\left(\left(x_{0} \mathbf{U}, x_{1} \mathbf{U}, x_{2} \mathbf{U}, x_{3} \mathbf{U}\right), g\right) \mapsto\left(\left(x_{0} \mathbf{B} x_{0}^{-1}, x_{1} \mathbf{B} x_{1}^{-1}, x_{2} \mathbf{B} x_{2}^{-1}, x_{3} \mathbf{B} x_{3}^{-1}\right), g\right) .
$$

We use this to identify ${ }^{\diamond} \mathcal{Y}=\dot{Z} \times{ }_{G} \dot{Z}$. Then $\pi_{1}^{\prime}, \pi_{2}^{\prime}$ become $d,{ }^{\diamond} \eta$ of 3.25 . We see that (a) holds.

5.10. Let $z \cdot \lambda \in \mathfrak{o}$. We set $\Sigma=\epsilon^{*} \zeta\left(R_{\lambda}^{\dot{z}}\right)\langle 2 \nu+|z|\rangle \in \mathcal{D}_{m}\left(\tilde{\mathcal{B}}^{2}\right)$. Let $j \in \mathbf{Z}$. We show:

(a) If $z \cdot \lambda \preceq \mathbf{c}$ then $\Sigma^{j} \in \mathcal{M} \preceq \tilde{\mathcal{B}}^{2}$.

(b) If $z \cdot \lambda \prec \mathbf{c}$ then $\Sigma^{j} \in \mathcal{M}^{\prec} \tilde{\mathcal{B}}^{2}$.

(c) If $z \cdot \lambda \in \mathbf{c}$ and $j>\nu+2 \rho+2 a$ then $\Sigma^{j} \in \mathcal{M}^{\prec} \tilde{\mathcal{B}}^{2}$.

If $z \cdot \lambda \notin W \mathfrak{s}$ then $\Sigma=0$ and there is nothing to prove. Now assume that $z \cdot \lambda \in W \mathfrak{s}$. Then, using $5.9($ a), we have

$$
\Sigma=\epsilon^{*} \zeta\left(\chi\left(\mathcal{L}_{\lambda}^{\dot{z} \sharp}\right)\right)\langle 2 \nu+|z|\rangle=\mathfrak{b}^{\prime}\left(\mathcal{L}_{\lambda}^{\dot{z} \sharp}\right)\langle 2 \nu+|z|\rangle=\mathfrak{b}^{\prime}\left(\mathbb{L}_{\lambda}^{\dot{z}}\right)\langle\nu-\rho\rangle .
$$

Now (a), (b) follow from 3.14(a), (b) and (c) follows from 3.14(c). (If $j>$ $\nu+2 \rho+2 a$ then $j+\nu-r>2 \nu+\rho+2 a$.) 
5.11. We show:

(a) If $K \in \mathcal{D}^{\preceq} G$ then $\zeta(K) \in \mathcal{D}^{\preceq} Z$.

(b) If $K \in \mathcal{D}^{\prec} G$ then $\zeta(K) \in \mathcal{D}^{\prec} Z$.

(c) If $K \in \mathcal{D}^{\preceq} G$ and $j>\nu+a$ then $(\zeta(K))^{j} \in \mathcal{M}^{\prec} Z$.

We can assume in addition that $K=A \in C S_{\mathfrak{o}}$ where $A \in C S_{\mathbf{c}^{\prime}}$ for a twosided cell $\mathbf{c}^{\prime}$ such that $\mathbf{c}^{\prime} \preceq \mathbf{c}$. Assume first that $\mathbf{c}^{\prime}=\mathbf{c}$. By $5.2(\mathrm{c})$ we can find $z \cdot \lambda \in \mathbf{c}$ such that $\left(A:\left(R_{\lambda}^{\dot{z}}\right)^{n_{z}}\right) \neq 0$. Then $A\left[-n_{z}\right]$ (without mixed structure) is a direct summand of $R_{\lambda}^{\dot{z}}$ (which is a semisimple complex). Hence $\epsilon^{*} \zeta(A)\left[-n_{z}\right]$ is a direct summand of $\epsilon^{*} \zeta\left(R_{\lambda}^{\dot{z}}\right)$ and $\epsilon^{*} \zeta(A)\left[-n_{z}+2 \nu+|z|\right]$ is a direct summand of $\Sigma$ (in 5.10), that is, $\epsilon^{*} \zeta(A)[-a-\rho]$ is a direct summand of $\Sigma$. By 5.10, if $j \in \mathbf{Z}$ (resp. $j>\nu+2 \rho+2 a$ ) then $\Sigma^{j} \in \mathcal{M} \preceq \tilde{\mathcal{B}}^{2}$ (resp. $\left.\Sigma^{j} \in \mathcal{M}^{\prec} \tilde{\mathcal{B}}^{2}\right)$ hence $\left(\epsilon^{*} \zeta(A)[-a-\rho]\right)^{j} \in \mathcal{M}^{\preceq} \tilde{\mathcal{B}}^{2}\left(\operatorname{resp} . \quad\left(\epsilon^{*} \zeta(A)[-a-\rho]\right)^{j} \in\right.$ $\left.\mathcal{M}^{\prec} \tilde{\mathcal{B}}^{2}\right)$, that is $\left(\epsilon^{*} \zeta(A)\right)^{j-a-\rho} \in \mathcal{M} \preceq \tilde{\mathcal{B}}^{2}$ (resp. $\left.\left(\epsilon^{*} \zeta(A)\right)^{j-a-\rho} \in \mathcal{M}^{\prec} \tilde{\mathcal{B}}^{2}\right)$.

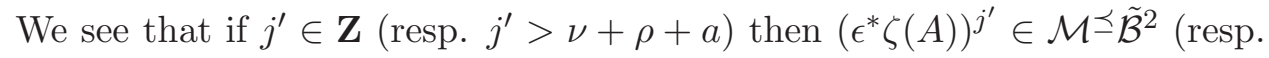
$\left.\left(\epsilon^{*} \zeta(A)\right)^{j^{\prime}} \in \mathcal{M}^{\prec} \tilde{\mathcal{B}}^{2}\right)$ so that $(\zeta(A))^{j^{\prime}-\rho} \in \mathcal{M}^{\prec} Z\left(\operatorname{resp} .(\zeta(A))^{j^{\prime}-\rho} \in \mathcal{M}^{\prec} Z\right)$; here we use 3.3(a). We see that if $j \in \mathbf{Z}$ (resp. $j>\nu+a$, so that $j+\rho>$ $\nu+\rho+a)$ then $(\zeta(A))^{j} \in \mathcal{M} Z$ (resp. $\left.(\zeta(A))^{j} \in \mathcal{M}^{\prec} Z\right)$. Thus the desired results hold when $\mathbf{c}^{\prime}=\mathbf{c}$.

Assume now that $\mathbf{c}^{\prime} \prec \mathbf{c}$. Applying the above argument with $\mathbf{c}$ replaced by $\mathbf{c}^{\prime}$ we see that (a), (b) hold.

5.12. For $K \in \mathcal{C}_{0}^{\mathbf{c}} G$ we set

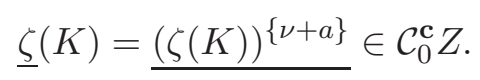

We say that $\underline{\zeta}(K)$ is the truncated restriction of $K$.

5.13. Let $L \in \mathcal{C}_{0}^{\mathbf{c}} Z$. We show:

(a) We have canonically $\underline{\zeta} \underline{\chi}(L))=\underline{\mathfrak{b}^{\prime \prime}}(L)$.

We shall apply [24, 1.12] with $\Phi: \mathcal{D}_{m}\left(Y_{1}\right) \rightarrow \mathcal{D}_{m}\left(Y_{2}\right)$ replaced by $\zeta$ : $\mathcal{D}_{m}(G) \rightarrow \mathcal{D}_{m}(Z)$ and with $\mathcal{D} \preceq\left(Y_{1}\right), \mathcal{D} \preceq\left(Y_{2}\right)$ replaced by $\mathcal{D} \preceq G, \mathcal{D} \preceq Z$. We shall take $\mathbf{X}$ in loc.cit. equal to $\chi(L)$. The conditions of loc.cit. are satisfied: those concerning $\mathbf{X}$ are satisfied with $c^{\prime}=a+\nu$, see 5.3. The conditions concerning $\zeta$ are satisfied with $c=a+\nu$, see 5.11. We see that 


$$
(\zeta(\chi(L)))^{j}=0 \text { if } j>2 a+2 \nu
$$

and

$$
\underline{g r_{2 a+2 \nu}\left((\zeta(\chi(L)))^{2 a+2 \nu}\right)}(a+\nu)=\underline{\zeta}(\underline{\chi}(L)) .
$$

Since $\zeta(\chi(L))=\mathfrak{b}^{\prime \prime}(L)$, we see that the left hand side of (c) equals $\underline{\mathfrak{b}^{\prime \prime}}(L)$. Thus (a) is proved.

Combining (a) with $3.25(\mathrm{~d})$ and $3.14(\mathrm{~d})$ we obtain the following result.

(b) We have canonically $\underline{\tilde{\epsilon}} \underline{\zeta}(\underline{\chi}(L))=\underline{\mathfrak{b}}(L)$.

5.14. Let $K \in \mathcal{D}_{m}(G)$ and let $L \in \mathcal{D}_{m}^{\mathbf{B}} \tilde{\mathcal{B}}^{2}$. We show that

(a) there is a canonical isomorphism $L \circ \epsilon^{*} \zeta(K) \stackrel{\sim}{\rightarrow} \epsilon^{*} \zeta(K) \circ L$.

Let $Y=\tilde{\mathcal{B}}^{2} \times G$. Define $j: Y \rightarrow G$ by $j\left(x_{0} \mathbf{U}, x_{1} \mathbf{U}, g\right)=g$. Define $j_{1}:$ $Y \rightarrow \tilde{\mathcal{B}}^{2}$ by $j_{1}\left(x_{0} \mathbf{U}, x_{1} \mathbf{U}, g\right)=\left(x_{0} \mathbf{U}, g^{-1} x_{1} \mathbf{U}\right)$. Define $j_{2}: Y \rightarrow \tilde{\mathcal{B}}^{2}$ by $j_{2}\left(x_{0} \mathbf{U}, x_{1} \mathbf{U}, g\right)=\left(g x_{0} \mathbf{U}, x_{1} \mathbf{U}\right)$. From the definitions we have $L \circ \epsilon^{*} \zeta(K)=$ $j_{2 !}\left(j_{1}^{*}(L) \otimes j^{*}(K)\right), \epsilon^{*} \zeta(K) \circ L=j_{2 !}\left(j_{2}^{*}(L) \otimes j^{*}(K)\right)$. By the $G$-equivariance of $L$ we have $j_{1}^{*} L=j_{2}^{*} L$; (a) follows.

Now let $K \in \mathcal{C}_{0}^{\mathbf{c}} G$ and let $L \in \mathcal{C}_{0}^{\mathbf{c}} \tilde{\mathcal{B}}^{2}$. We show that

(b) there is a canonical isomorphism $L \underline{\mathrm{o}} \underline{\tilde{\zeta}} \underline{(}(K) \stackrel{\sim}{\rightarrow}(\tilde{\epsilon} \underline{\zeta}(K)) \underline{\circ} L$.

We apply [24, 1.12] with $\Phi: \mathcal{D}_{\frac{\mathfrak{m}}{\mathfrak{B}}} \tilde{\mathcal{B}}^{2} \rightarrow \mathcal{D}_{m}^{\preceq} \tilde{\mathcal{B}}^{2}, L^{\prime} \mapsto L^{\prime} \circ L, \mathbf{X}=\tilde{\epsilon} \zeta(K)$ and with $\left(c, c^{\prime}\right)=(a-\nu, \nu+a)$, see $2.23(\mathrm{a}), 5.11(\mathrm{c})$. We deduce that we have canonically

$$
\underline{\left.\underline{\left((\tilde{\epsilon} \zeta(K))^{\{a+\nu\}}\right.} \circ L\right)^{\{a-\nu\}}}=\underline{(\tilde{\epsilon} \zeta(K) \circ L)^{\{2 a\}}} .
$$

We apply [24, 1.12] with $\Phi: \mathcal{D} \frac{\preceq}{\bar{m}} \tilde{\mathcal{B}}^{2} \rightarrow \mathcal{D}_{\bar{m}}^{\preceq} \tilde{\mathcal{B}}^{2}, L^{\prime} \mapsto L \circ L^{\prime}, \mathbf{X}=\tilde{\epsilon} \zeta(K)$ and with $\left(c, c^{\prime}\right)=(a-\nu, \nu+a)$, see $2.23(\mathrm{a}), 5.11(\mathrm{c})$. We deduce that we have canonically

$$
\underline{\left(\left(L \circ \underline{\left.(\tilde{\epsilon} \zeta(K))^{\{a+\nu\}}\right)^{\{a-\nu\}}}\right.\right.}=\underline{(L \circ \tilde{\epsilon} \zeta(K))^{\{2 a\}}} .
$$

We now combine (c), (d) with (a); we obtain (b). 
5.15. Let $\mu: G \times G \rightarrow G$ be the multiplication map. For $K, K^{\prime}$ in $\mathcal{D}(G)$ (resp. in $\left.\mathcal{D}_{m}(G)\right)$ we set $K * K^{\prime}=\mu_{!}\left(K \otimes K^{\prime}\right)$; this is in $\mathcal{D}(G)$ (resp. in $\left.\mathcal{D}_{m}(G)\right)$. For $K, K^{\prime}, K^{\prime \prime}$ in $\mathcal{D}_{m}(G)$ we have canonically $\left(K * K^{\prime}\right) * K^{\prime \prime}=$ $K *\left(K^{\prime} * K^{\prime \prime}\right)$ (and we denote this by $\left.K * K^{\prime} * K^{\prime \prime}\right)$. Note that if $K \in \mathcal{D}_{m}(G)$ and $K^{\prime} \in \mathcal{M}_{m}(G)$ is $G$-equivariant for the conjugation action of $G$ then as in $[24,4.1]$ we have a canonical isomorphism

$$
K * K^{\prime} \stackrel{\sim}{\rightarrow} K^{\prime} * K
$$

5.16. We show:

(a) For $K \in \mathcal{D}_{m}(G), L \in \mathcal{D}_{m}(Z)$ we have canonically $K * \chi(L)=\chi(L \bullet \zeta(K))$.

Let $Y=G \times G \times \mathcal{B}$. Define $c: Y \rightarrow G \times Z$ by $c\left(g_{1}, g_{2}, B\right)=\left(g_{1},\left(B, g_{2} B g_{2}^{-1}\right.\right.$, $\left.g_{2} U_{B}\right)$ ); define $d: Y \rightarrow G$ by $d\left(g_{1}, g_{2}, B\right)=g_{1} g_{2}$. From the definitions we see that both $K * \chi(L), \chi(L \bullet \zeta(K))$ can be identified with $d_{!} c^{*}(K \otimes L)$. This proves (a).

Now let $L, L^{\prime} \in \mathcal{D}_{m}(Z)$. Replacing in (a) $K, L$ by $\chi(L), L^{\prime}$ and using $5.9(\mathrm{a})$, we obtain

$$
\chi(L) * \chi\left(L^{\prime}\right)=\chi\left(L^{\prime} \bullet \mathfrak{b}^{\prime \prime}(L)\right)
$$

5.17. Let $L, L^{\prime} \in \mathcal{D}_{m}^{\mathbf{A}}(Z), j \in \mathbf{Z}$. We show:

(a) If $L \in \mathcal{D}^{\preceq} Z$ or $L^{\prime} \in \mathcal{D}^{\preceq} Z$ then $L^{\prime} \bullet \mathfrak{b}^{\prime \prime}(L) \in \mathcal{D}^{\preceq} Z$.

(b) If $L \in \mathcal{D}^{\prec} Z$ or $L^{\prime} \in \mathcal{D}^{\prec} Z$ then $L^{\prime} \bullet \mathfrak{b}^{\prime \prime}(L) \in \mathcal{D}^{\prec} Z$.

(c) If $L \in \mathcal{M} \preceq Z, L^{\prime} \in \mathcal{M}^{\bullet} Z$ and $j>3 a+\rho+\nu$ then $\left(L^{\prime} \bullet \mathfrak{b}^{\prime \prime}(L)\right)^{j} \in \mathcal{D}^{\prec} Z$.

Now (a), (b) follow from 3.25(b) and 3.23(a). To prove (c) we may assume that $L=\mathbb{L}_{\lambda}^{\dot{w}}, L^{\prime}=\mathbb{L}_{\lambda^{\prime}}^{\dot{w}^{\prime}}$ with $w \cdot \lambda, w^{\prime} \cdot \lambda^{\prime}$ in $W_{\mathfrak{s}}$ and $w \cdot \lambda \preceq \mathbf{c}$. We apply [24, 1.12] with $\Phi: \mathcal{D}_{\bar{m}}^{\prec} Z \rightarrow \mathcal{D}_{\bar{m}}^{\prec} Z, L_{1} \mapsto L^{\prime} \bullet L_{1}$ and $\mathbf{X}=\overline{\mathfrak{b}^{\prime \prime}}(L)$ and with $c^{\prime}=2 \nu+2 a\left(\right.$ see 3.25(c)), $c=a+\rho-\nu\left(\right.$ see 3.23(b)). We have $c+c^{\prime}=\nu+\rho+3 a$ hence (c) holds.

5.18. Let $L, L^{\prime} \in \mathcal{D}_{m}^{\mathbf{\$}}(Z), j \in \mathbf{Z}$. We show:

(a) If $L \in \mathcal{D}^{\preceq} Z$ or $L^{\prime} \in \mathcal{D}^{\preceq} Z$ then $\chi\left(L^{\prime} \bullet \mathfrak{b}^{\prime \prime}(L)\right) \in \mathcal{D}^{\preceq} G$.

(b) If $L \in \mathcal{D}^{\prec} Z$ or $L^{\prime} \in \mathcal{D}^{\prec} Z$ then $\chi\left(L^{\prime} \bullet \mathfrak{b}^{\prime \prime}(L)\right) \in \mathcal{D}^{\prec} G$. 
(c) If $L^{\prime} \in \mathcal{M}^{\natural} Z, L \in \mathcal{M}^{\preceq} Z$ and $j>4 a+2 \nu+\rho$ then $\left(\chi\left(L^{\prime} \bullet \mathfrak{b}^{\prime \prime}(L)\right)\right)^{j} \in$ $\mathcal{M}^{\prec} G$.

(a), (b) follow from 5.3(a) using 5.17(a), (b). To prove (c) we can assume that $L=\mathbb{L}_{\lambda}^{\dot{w}}, L^{\prime}=\mathbb{L}_{\lambda^{\prime}}^{\dot{w}^{\prime}}$ with $w \cdot \lambda, w^{\prime} \cdot \lambda^{\prime}$ in $\breve{W}$ and $w \cdot \lambda \preceq \mathbf{c}$. We apply 24, 1.12] with $\Phi: \mathcal{D}_{\bar{m}}^{\prec} Z \rightarrow \mathcal{D}_{\bar{m}}^{\prec} G, L_{1} \mapsto \chi\left(L_{1}\right), \mathbf{X}=L^{\prime} \bullet \mathfrak{b}^{\prime \prime}(L)$ and with $c^{\prime}=\nu+\rho+3 a($ see $5.17(\mathrm{c})), c=a+\nu($ see $5.3(\mathrm{~b}))$. We have $c+c^{\prime}=2 \nu+\rho+4 a$ hence (c) holds.

5.19. Let $K, K^{\prime} \in \mathcal{D}_{m}^{\mathbf{\$}}(G)$. We show:

(a) If $K \in \mathcal{D} \preceq G$ or $K^{\prime} \in \mathcal{D} \preceq G$ then $K * K^{\prime} \in \mathcal{D} \preceq G$.

(b) If $K \in \mathcal{D}^{\prec} G$ or $K^{\prime} \in \mathcal{D}^{\prec} G$ then $K * K^{\prime} \in \mathcal{D}^{\prec} G$.

(c) If $K \in \mathcal{D} \preceq G$ or $K^{\prime} \in \mathcal{D} \preceq G$ and $j>2 a+\rho$ then $\left(K * K^{\prime}\right)^{j} \in \mathcal{D}^{\prec} G$.

We can assume that $K=A \in C S_{\mathfrak{o}}, K^{\prime}=A^{\prime} \in C S_{\mathfrak{o}}$. Let $A^{\prime \prime} \in \mathcal{M}(G)$ be a composition factor of $\left(A * A^{\prime}\right)^{j}$. By 5.2(c) we can find $w \cdot \lambda \in \mathbf{c}_{A}$, $w^{\prime} \cdot \lambda^{\prime} \in \mathbf{c}_{A^{\prime}}$ such that $\left(A:\left(R_{\lambda}^{\dot{w}}\right)^{n_{w}}\right) \neq 0,\left(A^{\prime}:\left(R_{\lambda^{\prime}}^{\dot{w}^{\prime}}\right)^{n} w^{\prime}\right) \neq 0$. Then $A$ is a direct summand of $R_{\lambda}^{\dot{w}}\left[n_{w}\right]$ and $A^{\prime}$ is a direct summand of $R_{\lambda^{\prime}}^{\dot{w}^{\prime}}\left[n_{w^{\prime}}\right.$ (without mixed structures). Hence $A * A^{\prime}$ is a direct summand of

$$
R_{\lambda}^{\dot{w}} * R_{\lambda^{\prime}}^{\dot{w}^{\prime}}\left[a(w \cdot \lambda)+a\left(w^{\prime} \cdot \lambda^{\prime}\right)+|w|+\left|w^{\prime}\right|+2 \Delta\right]
$$

and $\left(A * A^{\prime}\right)^{j}$ is a direct summand of

$$
\begin{aligned}
& \left(R_{\lambda}^{\dot{w}} * R_{\lambda^{\prime}}^{\dot{w}^{\prime}}\left[|w|+\left|w^{\prime}\right|+2 \nu+2 \rho\right]\right)^{j+a(w \cdot \lambda)+a\left(w^{\prime} \cdot \lambda^{\prime}\right)+2 \nu} \\
& =\left(\chi\left(\mathbb{L}_{\lambda}^{\dot{w}}\right) * \chi\left(\mathbb{L}_{\lambda^{\prime}}^{\dot{w}^{\prime}}\right)\right)^{j+a(w \cdot \lambda)+a\left(w^{\prime} \cdot \lambda^{\prime}\right)+2 \nu} .
\end{aligned}
$$

Using $5.16(\mathrm{~b})$ we see that $\left(A * A^{\prime}\right)^{j}$ is a direct summand of

$$
\left(\chi\left(\mathbb{L}_{\lambda^{\prime}}^{\dot{w}^{\prime}} \bullet \mathfrak{b}^{\prime \prime}\left(\mathbb{L}_{\lambda}^{\dot{w}}\right)\right)^{j+a(w \cdot \lambda)+a\left(w^{\prime} \cdot \lambda^{\prime}\right)+2 \nu} .\right.
$$

Hence $A^{\prime \prime}$ is a composition factor of (d). Using 5.18(a) we see that $A^{\prime \prime} \in C S_{\mathfrak{o}}$ and that $\mathbf{c}_{A^{\prime \prime}} \preceq w \cdot \lambda$ and $\mathbf{c}_{A^{\prime \prime}} \preceq w^{\prime} \cdot \lambda^{\prime}$. In the setup of (a) we have $w \cdot \lambda \preceq \mathbf{c}$ or $w^{\prime} \cdot \lambda^{\prime} \preceq \mathbf{c}$ hence $\mathbf{c}_{A^{\prime \prime}} \leq \mathbf{c}$. Thus (a) holds. Similarly, (b) holds. In the setup of (c) we have $w \cdot \lambda \preceq \mathbf{c}$ and $w^{\prime} \cdot \lambda^{\prime} \preceq \mathbf{c}$. Hence $a(w \cdot \lambda) \geq a, a\left(w^{\prime} \cdot \lambda^{\prime}\right) \geq a$. 
Assume that $\mathbf{c}_{A^{\prime \prime}}=\mathbf{c}$. Since $A^{\prime \prime}$ is a composition factor of $(\mathrm{d})$, we see from 5.18 (c) that

$$
j+a(w \cdot \lambda)+a\left(w^{\prime} \cdot \lambda^{\prime}\right)+2 \nu \leq 4 a+2 \nu+\rho
$$

hence $j+2 a+2 \nu \leq 4 a+2 \nu+\rho$ and $j \leq 2 a+\rho$. This proves (c).

5.20. For $K, K^{\prime} \in \mathcal{C}_{0}^{\mathbf{c}} G$ we set

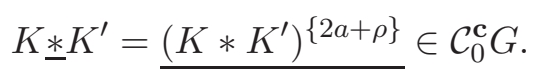

We say that $K \underline{*} K^{\prime}$ is the truncated convolution of $K, K^{\prime}$. Note that 5.15 (a) induces for $K, K^{\prime} \in \mathcal{C}_{0}^{\mathbf{c}} G$ a canonical isomorphism

$$
K \underline{*} K^{\prime} \stackrel{\sim}{\rightarrow} K^{\prime} \underline{*} K .
$$

Let $L \in \mathcal{C}_{0}^{\mathbf{c}} Z, K \in \mathcal{C}_{0}^{\mathbf{c}} G$. Using [24, 1.12] several times, we see that

$$
K_{\underline{*}} \underline{\chi}(L)=\underline{g r_{k}\left((K * \chi(L))^{k}\right)}(k / 2)
$$

where $k=(a+\nu)+(2 a+\rho)=3 a+\nu+\rho$ and

$$
\underline{\chi}(L \underline{\bullet} \underline{\zeta}(K))=\underline{g r_{k^{\prime}}\left(\left(\chi(L \bullet \zeta(K))^{k^{\prime}}\right)\right.}\left(k^{\prime} / 2\right)
$$

where $k^{\prime}=(a+\nu)+(a+\nu)+(a+\rho-\nu)=3 a+\nu+\rho$. Using now 5.16(a) and the equality $k=k^{\prime}$ we obtain

$$
K \underline{*} \underline{\chi}(L)=\underline{\chi}(L \underline{\bullet} \underline{\zeta}(K)) .
$$

Let $L, L^{\prime} \in \mathcal{C}_{0}^{\mathbf{c}} Z$. Using [24, 1.12] several times, we see that

$$
\underline{\chi}(L) \underline{*} \underline{\chi}\left(L^{\prime}\right)=\underline{g r_{k}\left(\left(\chi(L) * \chi\left(L^{\prime}\right)\right)^{k}\right)}(k / 2)
$$

where $k=(a+\nu)+(a+\nu)+(2 a+\rho)=4 a+2 \nu+\rho$ and

$$
\underline{\chi}\left(L^{\prime} \underline{\mathfrak{b}}^{\prime \prime}(L)=\underline{g r_{k^{\prime}}\left(\left(\chi\left(L^{\prime} \bullet \mathfrak{b}^{\prime \prime}(L)\right)\right)^{k^{\prime}}\right)}\left(k^{\prime} / 2\right)\right.
$$

where $k^{\prime}=(2 a+2 \nu)+(a+\rho-\nu)+(a+\nu)=4 a+2 \nu+\rho$. Using now 5.16(b) 
and the equality $k=k^{\prime}$ we obtain

$$
\underline{\chi}(L) \underline{*} \underline{\chi}\left(L^{\prime}\right)=\underline{\chi}\left(L^{\prime} \underline{\bullet}\left(\underline{\mathfrak{b}}^{\prime \prime}(L)\right)\right) .
$$

We show

(d) For $K, K^{\prime}, K^{\prime \prime}$ in $\mathcal{C}_{0}^{\mathbf{c}} G$ there is a canonical isomorphism $\left(K \underline{*} K^{\prime}\right) \underline{*} K^{\prime \prime} \stackrel{\sim}{\rightarrow} K_{\underline{*}}\left(K^{\prime} \underline{*} K^{\prime \prime}\right)$.

Indeed, just as in [24, 4.7] we can identify, using [24, 1.12], both $\left(K * K^{\prime}\right) * K^{\prime \prime}$ and $K \underline{*}\left(K^{\prime} \underline{*} K^{\prime \prime}\right)$ with $\left(K * K^{\prime} * K^{\prime \prime}\right)^{\{4 a+2 \rho\}}$.

A similar argument shows that the associativity isomorphism provided by (d) satisfies the pentagon property.

5.21. For $K, K^{\prime}$ in $\mathcal{D}_{m}(G)$ we show:

(a) We have canonically $\zeta\left(K * K^{\prime}\right)=\zeta\left(K^{\prime}\right) \bullet \zeta(K)$.

Let $Y=\left\{\left(B, g U_{B}, h_{1}, h_{2}\right) ; B \in \mathcal{B}, g \in G, h_{1} \in G, h_{2} \in G ; h_{1} h_{2} \in g U_{B}\right\}$. Define $j_{e}: Y \rightarrow G$ by $j_{e}\left(B, g U_{B}, h_{1}, h_{2}\right)=h_{e}(e=1,2)$. Define $j: Y \rightarrow$ $Z$ by $j\left(B, g U_{B}, h_{1}, h_{2}\right)=\left(B, g B g^{-1}, g U_{B}\right)$. From the definitions we have $\zeta\left(K * K^{\prime}\right)=j_{!}\left(j_{1}^{*}(K) \otimes j_{2}^{*}\left(K^{\prime}\right)\right)=\zeta\left(K^{\prime}\right) \bullet \zeta(K) ;$ (a) follows.

For $K, K^{\prime}$ in $\mathcal{D}_{0}^{\mathbf{c}}(G)$ we show:

(b) We have canonically $\underline{\zeta}\left(K \underline{*} K^{\prime}\right)=\underline{\zeta}\left(K^{\prime}\right) \underline{\bullet} \underline{\zeta}(K)$.

Using [24, 1.12] we see that

$$
\underline{\zeta}\left(K * K^{\prime}\right)=\underline{g r_{k}}\left(\left(\zeta\left(K * K^{\prime}\right)\right)^{k}\right)(k / 2)
$$

where $k=(a+\nu)+(2 a+\rho)=3 a+\nu+\rho$ and that

$$
\underline{\zeta}\left(K^{\prime}\right) \underline{\bullet} \underline{\zeta}(K)=\underline{g r_{k^{\prime}}\left(\left(\zeta(K) \bullet \zeta\left(K^{\prime}\right)\right)^{k^{\prime}}\right)}\left(k^{\prime} / 2\right)
$$

where $k^{\prime}=(a+\rho-\nu)+(a+\nu)+(a+\nu)=3 a+\nu+\rho$. It remains to use (a) and the equality $k=k^{\prime}$.

5.22. Define $h: G \rightarrow G g \mapsto g^{-1}$. For $K \in \mathcal{D}_{m}(G)$ we set $K^{\dagger}=h^{*} K$. We show: 
(a) For $L \in \mathcal{D}_{m}(Z)$ we have $(\chi(L))^{\dagger}=\chi\left(L^{\dagger}\right)$ with $L^{\dagger}$ as in 3.2 .

This follows from the definition of $\chi$ using the commutative diagram

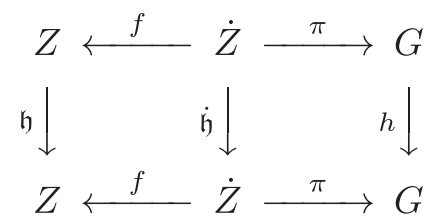

where $f, \pi$ are as in $5.1, \mathfrak{h}$ is as in 3.2 and $\dot{\mathfrak{h}}: \dot{Z} \rightarrow \dot{Z}$ is $\left(B, B^{\prime}, g\right) \mapsto$ $\left(B^{\prime}, B, g^{-1}\right)$.

From (a) and 3.2(a) we see that if $\lambda \in \mathfrak{s}, w \in W_{\lambda}^{\prime}$, then

$$
\left(\chi\left(\mathbb{L}_{\lambda}^{\dot{w}}\right)\right)^{\dagger}=\chi\left(\mathbb{L}_{\lambda^{-1}}^{\dot{w}^{-1}}\right)
$$

We deduce that

(c) if $A$ is a o-character sheaf with associated two-sided cell $\mathbf{c}$ then $A^{\dagger}$ is a $\mathfrak{o}^{-1}$-character sheaf with associated two-sided cell $\tilde{\mathbf{c}}$.

From (a), (c) we deduce:

(d) For $L \in \mathcal{C}_{0}^{\mathbf{c}} Z$ we have $(\underline{\chi}(L))^{\dagger}=\underline{\chi}\left(L^{\dagger}\right)$ where the second $\underline{\chi}$ is relative to $\tilde{\mathbf{c}}$ instead of $\mathbf{c}$.

\section{The Main Results}

6.1. Let $\mathbf{Y}=\{(B, g) ; B \in \mathcal{B}, g \in B\}$. We define $\mathfrak{k}: \mathbf{Y} \rightarrow \mathbf{T}$ by $(B, g) \mapsto t$ where $t \in \mathbf{T}$ is given by the conditions $x^{-1} g x \in t \mathbf{U}, x \in G, B=x \mathbf{B} x^{-1}$; note that $t$ is independent of the choice of $x$. Let

$$
\tilde{\mathbf{Y}}=\left\{((B, g), t) \in \mathbf{Y} \times \mathbf{T} ; \mathfrak{k}(B, g)=t^{n}\right\} .
$$

Let $G_{r s}$ be the variety of regular semisimple elements in $G$. Let

$$
\mathbf{Y}_{r s}=\left\{(B, g) \in \mathbf{Y} ; g \in G_{r s}\right\}, \tilde{\mathbf{Y}}_{r s}=\left\{((B, g), t) \in \tilde{\mathbf{Y}} ; g \in G_{r s}\right\}
$$

For any $w \in W$ we define $\tau_{w}: \mathbf{Y}_{r s} \rightarrow \mathbf{Y}_{r s}$ by $(B, g) \mapsto\left(B^{\prime}, g\right)$ where $B^{\prime} \in \mathcal{B}$ is uniquely defined by the condition that $g \in B^{\prime},\left(B^{\prime}, B\right) \in \mathcal{O}_{w}$; one verifies that $\tilde{\tau}_{w}: \tilde{\mathbf{Y}}_{r s} \rightarrow \tilde{\mathbf{Y}}_{r s},((B, g), t) \mapsto\left(\tau_{w}(B, g), w(t)\right)$, is well defined. Now 
$w \mapsto \tau_{w}$ (resp. $w \mapsto \tilde{\tau}_{w}$ ) is an action of $W$ on $\mathbf{Y}_{r s}$ (resp. on $\tilde{\mathbf{Y}}_{r s}$ ). For any $t_{1} \in \mathbf{T}_{n}$ we define $\tilde{\tau}^{t_{1}}: \tilde{\mathbf{Y}}_{r s} \rightarrow \tilde{\mathbf{Y}}_{r s}$ by $((B, g), t) \mapsto\left(\tau_{w}(B, g), t t_{1}\right)$. The operators $\tilde{\tau}_{w}, \tilde{\tau}^{t_{1}}$ define an action of the semidirect product $W \mathbf{T}_{n}$ (see 1.12) on $\tilde{\mathbf{Y}}_{r s}$. This action leaves stable each fibre of the map $\tilde{\varpi}_{r s}: \tilde{\mathbf{Y}}_{r s} \rightarrow G_{r s}$, $((B, g), t) \mapsto g$, hence it induces an action of $W \mathbf{T}_{n}$ on $\tilde{\varpi}_{r s !} \overline{\mathbf{Q}}_{l}$, a local system of rank $\sharp(W) n^{\rho}$ on $G_{r s}$ such that the induced $W \mathbf{T}_{n}$-action on any of its stalks is isomorphic to the regular representation of $W \mathbf{T}_{n}$. We show:

(a) The algebra homomorphism $h: \overline{\mathbf{Q}}_{l}\left[W \mathbf{T}_{n}\right] \rightarrow \operatorname{End}\left(\tilde{\varpi}_{r s !} \overline{\mathbf{Q}}_{l}\right)$ defined by the action above is an isomorphism.

Note that $h$ is injective since the induced algebra homomorphism from $\overline{\mathbf{Q}}_{l}\left[W \mathbf{T}_{n}\right]$ to the space of linear endomorphisms of any stalk of $\tilde{\varpi}_{r s !} \overline{\mathbf{Q}}_{l}$ is clearly injective. Since

$$
\operatorname{End}\left(\tilde{\varpi}_{r s !} \overline{\mathbf{Q}}_{l}\right) \cong \operatorname{Hom}_{\mathcal{D}\left(\tilde{\mathbf{Y}}_{r s}\right)}\left(\overline{\mathbf{Q}}_{l}, \tilde{\varpi}_{r s}^{*} \tilde{\varpi}_{r s !} \overline{\mathbf{Q}}_{l}\right),
$$

it is enough to show that

$$
\operatorname{dim} \operatorname{Hom}_{\mathcal{D}\left(\tilde{\mathbf{Y}}_{r s}\right)}\left(\overline{\mathbf{Q}}_{l}, \tilde{\varpi}_{r s}^{*} \tilde{\varpi}_{r s !} \overline{\mathbf{Q}}_{l}\right) \leq \sharp(W) n^{\rho} .
$$

Since $\tilde{\varpi}_{r s}^{*} \tilde{\varpi}_{r s !} \overline{\mathbf{Q}}_{l}$ is a local system of rank $\sharp(W) n^{\rho}$ on $\tilde{\mathbf{Y}}_{r s}$, it is enough to show that $\tilde{\mathbf{Y}}_{r s}$ is connected. Since $\tilde{\mathbf{Y}}_{r s} \rightarrow \mathcal{B},((B, g), t) \mapsto B$ is a $G$-equivariant fibration with $G$ acting transitively on $\mathcal{B}$, it is enough to show that its fibre over $\mathbf{B}$ is connected or that $\left\{(g, t) \in G_{r s} \times \mathbf{T} ; g \in t^{n} \mathbf{U}\right\}$ is connected, or that

$$
\left\{(t, u) \in \mathbf{T} \times \mathbf{U} ; t^{n} u \in G_{r s}\right\}=\left\{t \in \mathbf{T} ; t^{n} \in G_{r s}\right\} \times \mathbf{U}
$$

is connected. It is enough to observe that $\left\{t \in \mathbf{T} ; t^{n} \in G_{r s}\right\}$ is connected (it is a nonempty open subset of $\mathbf{T}$ ). This proves (a).

We define $\tilde{\varpi}: \tilde{\mathbf{Y}} \rightarrow G$ by $((B, g), t) \mapsto g$. We have $\tilde{\varpi}=\varpi \pi_{1}$ where $\pi_{1}: \tilde{\mathbf{Y}} \rightarrow \mathbf{Y}$ is $((B, g), t) \mapsto(B, g)$ and $\varpi: \mathbf{Y} \rightarrow G$ is $(B, g) \mapsto g$. From the cartesian diagram

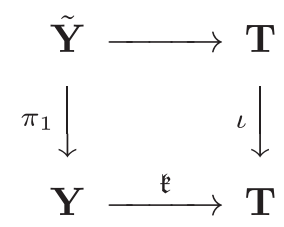


with $\iota: \mathbf{T} \rightarrow \mathbf{T}$ as in 1.4 , we see that $\mathfrak{k}^{*} \iota ! \overline{\mathbf{Q}}_{l}=\pi_{1 !} \overline{\mathbf{Q}}_{l}$, hence

$$
\Xi:=\widetilde{\varpi}_{!} \overline{\mathbf{Q}}_{l}=\varpi_{!}\left(\pi_{1 !} \overline{\mathbf{Q}}_{l}\right)=\varpi_{!}\left(\mathfrak{k}^{*} \iota ! \overline{\mathbf{Q}}_{l}\right)=\oplus_{\lambda \in \mathfrak{s}} \varpi_{!}\left(\mathfrak{k}^{*} L_{\lambda}\right)=\oplus_{l \in \mathfrak{s}} \Xi_{\lambda}
$$

where $\Xi_{\lambda}=\varpi_{!}\left(\mathfrak{k}^{*} L_{\lambda}\right)$. Since $\left.\Xi\right|_{G_{r s}}=\tilde{\varpi}_{r s !} \overline{\mathbf{Q}}_{l}$, we have $\tilde{\varpi}_{r s !} \overline{\mathbf{Q}}_{l}=\left.\oplus_{\lambda \in \mathfrak{s}} \Xi_{\lambda}\right|_{G_{r s}}$. As observed in [12], $\varpi: \mathbf{Y} \rightarrow G$ is small and $\Xi_{\lambda}$ is the intersection cohomology complex of $G$ with coefficients in $\left.\Xi_{\lambda}\right|_{G_{r s}}$; hence $\Xi$ is the intersection cohomology complex of $G$ with coefficients in $\tilde{\varpi}_{r s !} \overline{\mathbf{Q}}_{l}$. It follows that $\operatorname{End}_{\mathcal{D}(G)} \Xi=\operatorname{End}_{\mathcal{D}\left(G_{r s}\right)}\left(\tilde{\varpi}_{r s !} \overline{\mathbf{Q}}_{l}\right)$ hence, using (a),

$$
\operatorname{End}_{\mathcal{D}(G)} \Xi=\overline{\mathbf{Q}}_{l}\left[W \mathbf{T}_{n}\right]
$$

For any $E \in \operatorname{Irr}\left(W \mathbf{T}_{n}\right)$ we set

$$
A_{E}=\operatorname{Hom}_{\overline{\mathbf{Q}}_{l}\left[W \mathbf{T}_{n}\right]}(E, \Xi\langle\Delta\rangle) .
$$

We see that $A_{E}$ is a simple perverse sheaf on $G$ and that for $E \neq E^{\prime}$ in $\operatorname{Irr}\left(W \mathbf{T}_{n}\right)$ we have $A_{E} \not A_{E^{\prime}}$. Moreover we have

$$
\Xi\langle\Delta\rangle=\oplus_{E \in \operatorname{Irr}\left(W \mathbf{T}_{n}\right)} E \otimes A_{E}
$$

From the definitions, for $\lambda \in \mathfrak{s}$ we have $\Xi_{\lambda}=R_{\lambda}^{\mathrm{i}}$ (notation of 5.1). Using this and (d) we see that for any $E \in \operatorname{Irr}\left(W \mathbf{T}_{n}\right), A_{E}$ is a character sheaf on G.

We state the following result.

Proposition 6.2. For any $E^{\prime} \in \operatorname{Irr}\left(W \mathbf{T}_{n}\right)$ we have $\mathbf{c}_{A_{E^{\prime}}}=\mathbf{c}_{E^{\prime}}$. In particular, we have $a_{A_{E^{\prime}}}=a_{E^{\prime}}$. (Notation of $5.1,1.13$ ).

The proof is given in $6.3,6.4$, assuming, to simplify the exposition, that $n=1$. It consists in a reduction to an analogous (known) statement in the representation theory of the finite group $G^{F}$ in [13]. (We denote by $F: G \rightarrow G, F: \mathcal{B} \rightarrow \mathcal{B}$ the Frobenius maps corresponding to the $\mathbf{F}_{q^{-}}$ structures on $G, \mathcal{B}$.)

6.3. Until the end of 6.5 we assume that $n=1$. Then $W \mathbf{T}_{n}=W, \mathfrak{s}=\{1\}$ hence we can identify $W \mathfrak{s}=W ; \mathbf{H}$ has a basis $\left\{T_{w} ; w \in W\right\}$. As in [3], for each $w \in W$ we consider the variety $X_{w}=\left\{B \in \mathcal{B} ;(B, F(B)) \in \mathcal{O}_{w}\right\}$ on which $G^{F}$ acts by conjugation and the resulting $G^{F}$-module $H_{c}^{i}\left(X_{w}, \overline{\mathbf{Q}}_{l}\right)$ for 
each $i \in \mathbf{Z}$. Let $\operatorname{Irr}_{u}\left(G^{F}\right)$ be a set of representatives for the isomorphism classes of irreducible representations $\mathbf{r}$ of $G^{F}$ such that $\mathbf{r}$ appears in the $G^{F}$. module $H_{c}^{i}\left(X_{w}, \overline{\mathbf{Q}}_{l}\right)$ for some $w \in W$ and some $i \in \mathbf{Z}$, or equivalently (see [3]), such that $\sum_{i}(-1)^{i}\left(\mathbf{r}: H_{c}^{i}\left(X_{w}, \overline{\mathbf{Q}}_{l}\right)\right) \neq 0$ for some $w \in W$. (Here $(\mathbf{r}: ?)$ denotes the multiplicity of $\mathbf{r}$ in ?.) In the terminology of [3], $\operatorname{Irr}_{u}\left(G^{F}\right)$ is the set of unipotent representations of $G^{F}$. For any $\mathbf{r} \in \operatorname{Irr}_{u}\left(G^{F}\right)$ and any $E \in \operatorname{Irr}(W)$ we set

$$
b_{\mathbf{r}, E}=\sharp(W)^{-1} \sum_{w \in W} \operatorname{tr}(w, E) \sum_{i}(-1)^{i}\left(\mathbf{r}: H_{c}^{i}\left(X_{w}, \overline{\mathbf{Q}}_{l}\right)\right) \in \mathbf{Q} .
$$

By [13, 4.23], given $\mathbf{r} \in \operatorname{Irr}_{u}\left(G^{F}\right)$, there is a unique two-sided cell $\mathbf{c}_{\mathbf{r}}$ of $W$ such that $b_{\mathbf{r}, E}=0$ whenever $E \in \operatorname{Irr}(W)$ satisfies $\mathbf{c}_{E} \neq \mathbf{c}_{\mathbf{r}}$; let $a_{\mathbf{r}}$ be the value of the $a$-function $a: W \rightarrow \mathbf{N}$ on $\mathbf{c}_{\mathbf{r}}$.

For $w=1$, we have $X_{1}=\mathcal{B}^{F}$ and $\mathcal{F}:=H_{c}^{0}\left(X_{1}, \overline{\mathbf{Q}}_{l}\right)$ is the vector space of functions $\mathcal{B}^{F} \rightarrow \overline{\mathbf{Q}}_{l}$. This vector space is naturally an $G^{F}$-module and its space of $G^{F}$-equivariants endomorphism can be naturally identified with the semisimple algebra $\mathbf{H}^{\sqrt{q}}:=\overline{\mathbf{Q}}_{l} \otimes_{\mathcal{A}} \mathbf{H}$ where $\overline{\mathbf{Q}}_{l}$ is viewed as an $\mathcal{A}$-algebra via $v \mapsto \sqrt{q}$. Hence for any simple $\mathbf{H}^{\sqrt{q}}$-module $M$, the vector space $\mathbf{r}_{M}=$ $\operatorname{Hom}_{\mathbf{H} \sqrt{q}}(M, \mathcal{F})$ is either 0 or an object of $\operatorname{Irr}_{u}\left(G^{F}\right)$; in fact, it is known that it is $\neq 0$. For any $E \in \operatorname{Irr}(W)$ let $E^{\sqrt{q}}$ be the simple $\mathbf{H}^{{ }^{q}}$-module corresponding to $E$ under the algebra isomorphisms $\mathbf{H} \sqrt{q} \stackrel{\psi^{\sqrt{q}}}{\rightarrow} \overline{\mathbf{Q}}_{l} \otimes \mathbf{H}^{\infty} \stackrel{\left(\psi^{1}\right)^{-1}}{\rightarrow} \mathbf{H}^{1}=\overline{\mathbf{Q}}_{l}[W]$ obtained by extension of scalars from $\psi: \mathbf{H} \rightarrow \mathcal{A} \otimes \mathbf{H}^{\infty}$ (see 1.12); we write $\mathbf{r}_{E}$ instead of $\mathbf{r}_{E \sqrt{q}}$. Thus we have an imbedding $\operatorname{Irr}(E) \rightarrow \operatorname{Irr}_{u}\left(G^{F}\right)$, $E \mapsto \mathbf{r}_{E}$.

6.4. We write $\mathfrak{R}^{\dot{z}}$ instead of $\mathfrak{R}_{\lambda}^{\dot{z}}$ (see 5.1) where $\lambda=1, z \in W$. The following result can be deduced from $[9,2.1]$.

(a) Let $y \in W, E^{\prime} \in \operatorname{Irr}(W), i \in \mathbf{Z}$. Then $\left.\mathcal{H}^{i} \Re^{i}\right|_{G_{r s}}$ is a local system and

$$
\sum_{i}(-1)^{i}\left(\left.A_{E^{\prime}}[-\Delta]\right|_{G_{r s}}:\left.\mathcal{H}^{i} \Re^{\dot{y}}\right|_{G_{r s}}\right)=\sum_{i}(-1)^{i}\left(\mathbf{r}_{E^{\prime}}: H_{c}^{i}\left(X_{y}, \overline{\mathbf{Q}}_{l}\right)\right) .
$$

(In the left hand side, (:) denotes the multiplicity of an irreducible local system on $G_{r s}$ in another local system on $G_{r s}$.) 
Using 6.3(a) we deduce for any $E, E^{\prime}$ in $\operatorname{Irr}(W)$ :

$$
b_{\mathbf{r}_{E^{\prime}}, E}=\sharp(W)^{-1} \sum_{y \in W} \operatorname{tr}(y, E) \sum_{i}(-1)^{i}\left(\left.A_{E^{\prime}}[-\Delta]\right|_{G_{r s}}:\left.\mathcal{H}^{i} \mathfrak{R}^{\dot{y}}\right|_{G_{r s}}\right) .
$$

From [16, (14.10.1)], for any $E \in \operatorname{Irr}(W), A \in C S_{\mathfrak{o}}$ we have

$$
b_{A, E}=\sharp(W)^{-1} \sum_{y \in W} \operatorname{tr}(y, E) \sum_{i}(-1)^{i+\Delta}\left(A:\left(\mathfrak{R}^{\dot{y}}\right)^{i}\right) .
$$

$\left(b_{A, E}\right.$ is as in 5.1(a).) In particular for $E^{\prime} \in \operatorname{Irr}(W)$ we have

$$
\begin{aligned}
& b_{A_{E^{\prime}}, E}=\sharp(W)^{-1} \sum_{y \in W} \operatorname{tr}(y, E) \sum_{i}(-1)^{i+\Delta}\left(A_{E^{\prime}}:\left(\Re^{\dot{y}}\right)^{i}\right) \\
= & \sharp(W)^{-1} \sum_{y \in W} \operatorname{tr}\left(y^{-1}, E\right) \sum_{i}(-1)^{i+\Delta}\left(\left.A_{E^{\prime}}\right|_{G_{r s}}:\left.\left(\Re^{\dot{y}}\right)^{i}\right|_{G_{r s}}\right) .
\end{aligned}
$$

Since $\mathcal{H}^{i} \mathfrak{R}_{G_{r s}}^{\dot{y}}$ are local systems, we see that

$$
\sum_{i}(-1)^{i+\Delta}\left(\left.A_{E^{\prime}}\right|_{G_{r s}}:\left.\left(\mathfrak{R}^{\dot{y}}\right)^{i}\right|_{G_{r s}}\right)=\sum_{i}(-1)^{i}\left(\left.A_{E^{\prime}}[\Delta]\right|_{G_{r s}}:\left.\mathcal{H}^{i} \mathfrak{R}^{\dot{y}}\right|_{G_{r s}}\right)
$$

(in the last sum (:) refers to multiplicities of an irreducible local system in another local system). Thus,

$$
b_{A_{E^{\prime}}, E}=\sharp(W)^{-1} \sum_{y \in W} \operatorname{tr}(y, E) \sum_{i}(-1)^{i}\left(\left.A_{E^{\prime}}[\Delta]\right|_{G_{r s}}:\left.\mathcal{H}^{i} \mathfrak{R}^{\dot{y}}\right|_{G_{r s}}\right)
$$

so that, using (b), we have

$$
b_{A_{E^{\prime}}, E}=b_{\mathbf{r}_{E^{\prime}}, E} .
$$

Using the definitions we now see that

$$
\mathbf{c}_{A_{E^{\prime}}}=\mathbf{c}_{\mathbf{r}_{E^{\prime}}} \text {, hence } a_{A_{E^{\prime}}}=a_{\mathbf{r}_{E^{\prime}}}
$$

for any $E^{\prime} \in \operatorname{Irr}(W)$. Thus we can restate 6.2 as follows:

(c) For any $E^{\prime} \in \operatorname{Irr}(W)$ we have $\mathbf{c}_{\mathbf{r}_{E^{\prime}}}=\mathbf{c}_{E^{\prime}}$.

We shall deduce (c) from the following result which is equivalent to 13 , $12.2(\mathrm{i})]$ : 
(d) Let $\Lambda$ be a left cell of $W$. Write $[\Lambda]=\oplus_{E \in \operatorname{Irr}(W)} E^{\oplus f_{E}}$ where $f_{E} \in \mathbf{N}$. In the Grothendieck module of $G^{F}$-modules tensored by $\mathbf{Q}$ we have

$$
\sum_{E \in \operatorname{Irr}(W)} f_{E} \mathbf{r}_{E}=\sum_{E \in \operatorname{Irr}(W)} \sum_{\mathbf{r} \in \operatorname{Irr}_{u} G^{F}} b_{\mathbf{r}, E} \mathbf{r}
$$

Let $E^{\prime} \in \operatorname{Irr}(W)$. We can find a left cell $\Lambda$ of $W$ as in (d) such that $\Lambda \subset \mathbf{c}_{E^{\prime}}$ and $E^{\prime}$ appears in $\Lambda$ ], that is, $f_{E^{\prime}}>0$. Then $\mathbf{r}_{E^{\prime}}$ appears with nonzero coefficient in the left hand side of the identity in (d) hence it appears with nonzero coefficient in the right hand side of the identity in (d). Thus there exists $E \in \operatorname{Irr}(W)$ such that $b_{\mathbf{r}_{E^{\prime}}, E} \neq 0$. By definition this means that $\mathbf{c}_{\mathbf{r}_{E^{\prime}}}=\mathbf{c}_{E^{\prime}}$, proving (c), hence also Proposition 6.2 (assuming $n=1$ ). The proof for general $n$ goes along similar lines.

Note that the proof of $6.4(\mathrm{~d})$ given in $[13,12.2(\mathrm{i})]$ is case by case. It is likely that a more efficient proof can be obtained using the inductive description of $W$-modules carried by left cells in terms of constructible representations given in [11].

6.5. The following inequality is a special case of Proposition 6.2.

(a) For any $E^{\prime} \in \operatorname{Irr}(W)$ we have $a_{A_{E^{\prime}}} \leq a_{E^{\prime}}$.

We give an alternative proof of (a) which avoids the use of 6.4(d) hence of [13, 12.2(i)]. We again assume for simplicity that $n=1$. As in 6.4 it is enough to prove:

(b) $a_{\mathbf{r}_{E^{\prime}}} \leq a_{E^{\prime}}$

It is known [5, 8.1.8] that

$$
\operatorname{dim}\left(\mathbf{r}_{E^{\prime}}\right)=\operatorname{dim}\left(E^{\prime}\right) \sum_{w \in W} q^{|w|}\left(\sum_{w \in W} q^{-|w|} \operatorname{tr}\left(T_{w}, E^{\prime \sqrt{q}}\right)^{2}\right)^{-1} .
$$

From [8, 3.14, 3.16, 3.17, 3.19] we have

$$
\operatorname{dim} \mathbf{r}_{E^{\prime}}=\sharp(W)^{-1} \sum_{E \in \operatorname{Irr}(W)} b_{\mathbf{r}_{E^{\prime}}, E} \sum_{i \geq 0} \operatorname{dim} \operatorname{Hom}_{W}\left(E, \bar{S}^{i} V\right) q^{i}
$$


Since $b_{\mathbf{r}_{E^{\prime}}, E}=0$ unless $\mathbf{c}_{E}=\mathbf{c}_{\mathbf{r}_{E^{\prime}}}$, it follows that

$$
\begin{aligned}
& \operatorname{dim}\left(E^{\prime}\right) \sum_{w \in W} q^{|w|}\left(\sum_{w \in W} q^{-|w|} \operatorname{tr}\left(T_{w}, E^{\prime \sqrt{q}}\right)^{2}\right)^{-1} \\
& =\sharp(W)^{-1} \sum_{E \in \operatorname{Irr}(W) ; \mathbf{c}_{E}=\mathbf{c}_{\mathbf{r}_{E^{\prime}}}} b_{\mathbf{r}_{E^{\prime}}, E} \sum_{i \geq 0} \operatorname{dim} \operatorname{Hom}_{W}\left(E, \bar{S}^{i} V\right) q^{i} .
\end{aligned}
$$

Since $b_{\mathbf{r}_{E^{\prime}}, E}=b_{A_{E^{\prime}}, E}$ is independent of $q$, we may regard (c) as an equality of polynomials with rational coefficients in an indeterminate $q^{1 / 2}$. From $1.20\left(\mathrm{c}^{\prime}\right)$ we see that the right hand side of (c) is in $q^{c} \mathbf{Q}\left[q^{-1 / 2}\right]$ where

$$
c=\max _{E ; \mathbf{c}_{E}=\mathbf{c}_{\mathbf{r}_{E^{\prime}}}}\left(\nu-b_{E \otimes \mathbf{s g n}}\right)=\nu-a_{\mathbf{r}_{E^{\prime}}}
$$

(the last equality uses 1.19(a), (b)). From [18, 20.11] we see that the left hand side of (c) is in $q^{\nu-a_{E^{\prime}}}\left(c_{0}+q^{-1 / 2} \mathbf{Q}\left[q^{-1 / 2}\right]\right)$ where $c_{0} \in \mathbf{Q}-\{0\}$. Hence from (c) we deduce that $\nu-a_{E^{\prime}} \leq \nu-a_{\mathbf{r}_{E^{\prime}}}$ so that $a_{E^{\prime}} \geq a_{\mathbf{r}_{E^{\prime}}}$, as required.

6.6. We now return to our general $n$. Let $A$ be a character sheaf of $G$. By [19, 30.12], there exists a parabolic subgroup $P$ of $G$, a Levi subgroup $L$ of $P$ and a subset $S_{1}$ of $L$ which is a single conjugacy class of $L$ times the connected centre of $L$ such that the support of $A$ is the union of $G$ conjugates of elements in the closure of $S_{1}$ times the unipotent radical of $P$; moreover, if $P \in \mathcal{B}$ then $A=A_{E}$ for some $E \in \operatorname{Irr}\left(W \mathbf{T}_{n}\right)$ while if $P \notin \mathcal{B}$ then $\left.A\right|_{\{1\}}=0$. (Here we use the cleanness of cuspidal character sheaves, see [23] and its references. Actually we only use a weak form of the cleanness property which is more elementary than what appears in [23].)

6.7. Let $\phi: \mathbf{p} \rightarrow G$ be the map with image 1 . For any $K \in \mathcal{D}_{m}(G)$ we have

$$
\left(\phi^{*} K\right)^{j}=\mathcal{H}_{1}^{j} K
$$

The identification 6.1(b) induces for any $i$ an algebra homomorphism

$$
\overline{\mathbf{Q}}_{l}\left[W \mathbf{T}_{n}\right] \rightarrow \operatorname{End}\left(\mathcal{H}_{1}^{i} \Xi\right)
$$

thus,

$$
\mathcal{H}_{1}^{i} \Xi=H_{c}^{i}\left(\tilde{\varpi}^{-1}(1), \overline{\mathbf{Q}}_{l}\right)=H_{c}^{i}\left(\mathcal{B} \times \mathbf{T}_{n}, \overline{\mathbf{Q}}_{l}\right)=H_{c}^{i}\left(\mathcal{B}, \overline{\mathbf{Q}}_{l}\right) \otimes \overline{\mathbf{Q}}_{l}\left[\mathbf{T}_{n}\right]
$$


becomes naturally a $W \mathbf{T}_{n}$-module; one verifies that the action of $w t$ (with $\left.w \in W, t \in \mathbf{T}_{n}\right)$ is given by $w t: e \otimes t_{1} \mapsto w(e) \otimes w\left(t t_{1}\right)$ (here $e \in H^{i}\left(\mathcal{B}, \overline{\mathbf{Q}}_{l}\right)$, $t_{1} \in \mathbf{T}_{n}$ and $e \mapsto w(e)$ is the $W$-action on $H^{i}\left(\mathcal{B}, \overline{\mathbf{Q}}_{l}\right)=H^{i}\left(G / \mathbf{B}, \overline{\mathbf{Q}}_{l}\right)=$ $H^{i}\left(G / \mathbf{T}, \overline{\mathbf{Q}}_{l}\right)$ induced by the conjugation action of $N \mathbf{T}$. (In the case where $n=1$ this is proved as in [29, §2]; the proof in the general case is along similar lines.) Note also that $\mathcal{H}_{1}^{i} \Xi=0$ if $i$ is odd. We show:

(a) Let $E$ be an irreducible $W \mathbf{T}_{n}$-module. We have $\left(\mathcal{H}_{1}^{2 i} \Xi\right)^{E}=0$ for $i>$ $\nu-a_{E}$. Moreover, $\operatorname{dim}\left(\mathcal{H}_{1}^{2 \nu-2 a_{E}} \Xi\right)^{E}$ is 1 if $E$ is special and 0 if $E$ is not special.

Let $\bar{S} V=\oplus_{i \geq 0} \bar{S}^{i} V$ be as in 1.20. It is well known that for $i \geq 0$ we have canonically $\bar{S}^{i} V=H_{c}^{2 i}\left(\mathcal{B}, \overline{\mathbf{Q}}_{l}\right)$ compatibly with the $W$-actions. This extends to an identification

$$
\bar{S}^{i} V \otimes \overline{\mathbf{Q}}_{l}\left[W \mathbf{T}_{n}\right]=H_{c}^{2 i}\left(\mathcal{B}, \overline{\mathbf{Q}}_{l}\right) \otimes \overline{\mathbf{Q}}_{l}\left[W \mathbf{T}_{n}\right]=\mathcal{H}_{1}^{2 i} \Xi
$$

which is compatible with the $W \mathbf{T}_{n}$-actions. Hence (a) follows from 1.21(a).

6.8. We show:

(a) Let $A \in C S_{\mathbf{c}}$. If $j>-2 a-\rho$ then $\left(\phi^{*} A\right)^{j}=\mathcal{H}_{1}^{j} A=0$.

We can assume that $A \cong A_{E}$ for some $E \in \operatorname{Irr}\left(W \mathbf{T}_{n}\right)$. (If $A$ is not of this form, the result holds by 6.6.) Since $a \leq a_{E}$ (see 6.5(a)) we have $j>$ $-2 a_{E}-\rho$. By definition we have $\mathcal{H}_{1}^{j} A_{E}=\left(\mathcal{H}_{1}^{j}(\Xi\langle\Delta\rangle)\right)^{E}=\left(\mathcal{H}_{1}^{j+\Delta} \Xi\right)^{E}(\Delta / 2)$ hence by $6.7(\mathrm{a}), \mathcal{H}_{1}^{j} A_{E}=0$ if $j+\Delta>2 \nu-2 a_{E}$ that is if $j>-2 a_{E}-\rho$.

We show:

(b) Let $E=E_{\mathbf{c}}$, see 1.19. Then $\left(\phi^{*} A_{E}\right)^{-2 a-\rho}=\mathcal{H}_{1}^{-2 a-\rho} A_{E}$ is a 1-dimensional mixed vector space of pure weight $-2 a-\rho$.

As in the proof of (a) we have

$$
\mathcal{H}_{1}^{-2 a-\rho} A_{E}=\left(\mathcal{H}_{1}^{-2 a-\rho}(\Xi\langle\Delta\rangle)\right)^{E}=\left(\mathcal{H}_{1}^{-2 a+2 \nu} \Xi\right)^{E}(\Delta / 2)
$$

By 6.7(a) we have $\operatorname{dim}\left(\mathcal{H}_{1}^{2 \nu-2 a} \Xi\right)^{E}=1$. It remains to note that $\left(\mathcal{H}^{2 \nu-2 a} 1 q \Xi\right)^{E}$ is pure of weight $2 \nu-2 a$ (indeed, $\mathcal{H}_{1}^{2 \nu-2 a} \Xi=H_{c}^{2 \nu-2 a}\left(\mathcal{B}, \overline{\mathbf{Q}}_{l}\right) \otimes \overline{\mathbf{Q}}_{l}\left[\mathbf{T}_{n}\right]$ is pure of weight $2 \nu-2 a$ ). 
We show:

(c) Let $A \in C S_{\mathbf{c}}$ be such that $A \nRightarrow A_{E_{\mathbf{c}}}$. Then $\left(\phi^{*} A\right)^{-2 a-\rho}=\mathcal{H}_{1}^{-2 a-\rho} A=0$.

As in the proof of (a) we can assume that $A \cong A_{E}$ for some $E \in \operatorname{Irr}\left(W \mathbf{T}_{n}\right)$. By 6.2 we have $\mathbf{c}_{E}=\mathbf{c}$ and by assumption we have $E \not E_{\mathbf{c}}$. Hence $E$ is not special. By $6.7(\mathrm{a})$ we have $\left(\mathcal{H}_{1}^{2 \nu-2 a_{E}} \Xi\right)^{E}=0$ hence $\mathcal{H}_{1}^{-2 a_{E}-\rho} A_{E}=0$. We have $a_{E}=a_{\mathbf{c}}=a$ hence $\mathcal{H}_{1}^{-2 a-\rho} A_{E}=0$. This proves (c).

From (b), (c) we see that if $K \in \mathcal{C}^{\mathbf{c}} G$ then

(d) $\operatorname{dim} \operatorname{Hom}_{\mathcal{C}^{\mathrm{c}} G}\left(A_{E_{\mathbf{c}}}, K\right)=\operatorname{dim}\left(\phi^{*} K\right)^{-2 a-\rho}=\operatorname{dim} \mathcal{H}_{1}^{-2 a-\rho} K$.

6.9. Let $\delta_{0}: \mathcal{B} \rightarrow Z, \pi_{0}^{\prime}: \mathcal{B} \rightarrow \mathbf{p}$ be as in 4.12 . We show that for $L \in \mathcal{D}_{m}(Z)$ we have

(a) $\phi^{*} \chi(L)=\pi_{0 !}^{\prime} \delta_{0}^{*} L$.

Define $\phi^{\prime}: \mathcal{B} \rightarrow \dot{Z}$ (see 4.1 ) by $B \mapsto(B, B, 1)$. We have a commutative diagram in which the right square is cartesian:

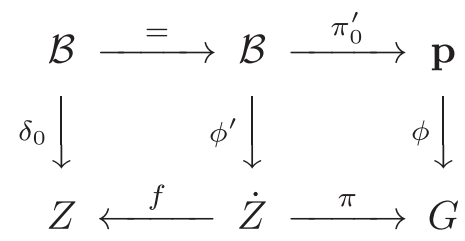

(Here $f, \pi$ are as in 4.1.) It follows that for $L \in \mathcal{D}_{m}(Z)$ we have $\phi^{*} \chi(L)=$ $\phi^{*} \pi_{!} f^{*} L=\pi_{0 !}^{\prime} \phi^{\prime *} f^{*} L=\pi_{0 !}^{\prime} \delta_{0}^{*} L$. This proves (a).

Let $L \in \mathcal{C}_{0}^{\mathbf{c}} Z$. Applying [24, 8.2] with $\Phi: \mathcal{D}_{m}^{\preceq} G \rightarrow \mathcal{D}_{m} \mathbf{p}, K_{1} \mapsto \phi^{*} K_{1}$, $c=-2 a-\rho($ see $6.8(\mathrm{a})), K$ replaced by $\chi(L)$ and $c^{\prime}=a+\nu$, we see that we have canonically

$$
\left(\phi^{*}(\underline{\chi}(L))\right)^{\{-2 a-\rho\}} \subset\left(\phi^{*} \chi(L)\right)^{\{-a-\rho+\nu\}}=\left(\pi_{0 !}^{\prime} \delta_{0}^{*}(L)\right)^{\{-a-\rho+\nu\}} .
$$

(The last equality follows from (a).) By 4.12(c), $\delta_{0}^{*} L$ is pure of weight 0 hence $\pi_{0 !}^{\prime} \delta_{0}^{*} L$ is pure of weight 0 hence $\left(\pi_{0 !}^{\prime} \delta_{0}^{*} L\right)^{\nu-a-\rho}$ is pure of weight $\nu-a-\rho$ so that

$$
\left(\pi_{0 !}^{\prime} \delta_{0}^{*} L\right)^{\{-a-\rho+\nu\}}=\left(\pi_{0 !}^{\prime} \delta_{0}^{*} L\right)^{-a-\rho+\nu}((-a-\rho+\nu) / 2)
$$


From 4.12(f) we have $\left(\pi_{0 !}^{\prime} \delta_{0}^{*} L\right)^{\nu-a-\rho}=\left(\pi_{0 !}^{\prime}\left(\left(\delta_{0}^{*} L\right)^{-a-\rho}\right)\right)^{\nu}$. Hence

$$
\left(\pi_{0 !}^{\prime} \delta_{0}^{*} L\right)^{\{-a-\rho+\nu\}}=\left(\pi_{0 !}^{\prime}\left(\left(\delta_{0}^{*} L\right)^{-a-\rho}\right)\right)^{\nu}((-a-\rho+\nu) / 2) .
$$

Thus (b) becomes

$$
\left(\phi^{*}(\underline{\chi}(L))\right)^{\{-2 a-\rho\}} \subset\left(\pi_{0 !}^{\prime}\left(\left(\delta_{0}^{*} L\right)^{-a-\rho}\right)\right)^{\nu}((-a-\rho+\nu) / 2)
$$

and using 4.12(h):

$$
\left(\phi^{*}(\underline{\chi}(L))\right)^{\{-2 a-\rho\}} \subset \operatorname{Hom}_{\mathcal{C}^{\mathbf{c}} Z}\left(\mathbf{1}_{0}^{\prime}, L\right) .
$$

We show that (c) is an equality:

$$
\left(\phi^{*}(\underline{\chi}(L))\right)^{\{-2 a-\rho\}}=\operatorname{Hom}_{\mathcal{C}^{\mathbf{c}} Z}\left(\mathbf{1}_{0}^{\prime}, L\right) .
$$

To prove this we can assume that $L=\mathbb{L}_{\lambda}^{\dot{w}}$ for some $w \cdot \lambda \in \breve{\mathbf{c}}$. If $w \cdot \lambda \notin \mathbf{D}_{\mathbf{c}}$ then the right hand side of $(d)$ is zero, hence by $(c)$, the left hand side of $(d)$ is zero and ( $\mathrm{d}$ ) holds. Assume now that $w \cdot \lambda \in \mathbf{D}_{\mathbf{c}}$. Then the right hand side of (c) has dimension 1 . Hence the left hand side of (c) has dimension 0 or 1 ; it is enough to prove that it has dimension 1. By $6.8(\mathrm{~d})$ with $K=\underline{\chi}(L)$ we see that the left hand side of (c) has dimension equal to $\left(A_{E_{\mathbf{c}}}: \underline{\chi}(L)\right)$. (We have also used 6.8(b).) In particular we have

$$
\left(A_{E_{\mathbf{c}}}: \underline{\chi}\left(\mathbb{L}_{\lambda}^{\dot{w}}\right)\right) \text { is } 0 \text { or } 1
$$

and we must prove that

$$
\left(A_{E_{\mathbf{c}}}: \underline{\chi}\left(\mathbb{L}_{\lambda}^{\dot{w}}\right)\right)=1
$$

In the rest of the proof we set $A=A_{E_{\mathbf{c}}}$. Using 5.8(a) we can reformulate (e) as $b_{A,\left[\Lambda_{w, \lambda}\right]} \in\{0,1\}$ for any $w \cdot \lambda \in \mathbf{D}_{\mathbf{c}}$; we must prove that $b_{A,\left[\Lambda_{w, \lambda}\right]}=1$ for any $w \cdot \lambda \in \mathbf{D}_{\mathbf{c}}$. Since $\mathbf{c}_{A}=\mathbf{c}$ we have $b_{A,[\Lambda]}=0$ for any left cell $\Lambda$ not contained in c. Hence it is enough to show that $\sum_{\Lambda} b_{A,[\Lambda]}=\sharp\left(\mathbf{D}_{\mathbf{c}}\right)$ where $\Lambda$ runs over the left cells in $W \mathfrak{s}$. We have $\sum_{\Lambda} b_{A,[\Lambda]}=b_{A, R e g}$ where $R e g$ is the regular representation of $W \mathbf{T}_{n}$. Hence it is enough to show that $b_{A, R e g}=\sharp\left(\mathbf{D}_{\mathbf{c}}\right)$. From 5.1(a) with $z=1, j=\Delta$ we have

$$
\sum_{\lambda \in \mathfrak{s}}\left(A:\left(R_{\lambda}^{1}\right)^{\Delta}\right)=\left(0 ; \sum_{E \in \operatorname{Irr}\left(W \mathbf{T}_{n}\right)} b_{A, E} \sum_{\lambda \in \mathfrak{s}} \operatorname{tr}\left(1_{\lambda}, E^{v}\right)\right)
$$




$$
=\left(0 ; \sum_{E \in \operatorname{Irr}\left(W \mathbf{T}_{n}\right)} b_{A, E} \operatorname{dim}(E)\right)=b_{A, R e g} .
$$

Hence it is enough to show that $\sum_{\lambda \in \mathfrak{s}}\left(A:\left(R_{\lambda}^{1}\right)^{\Delta}\right)=\sharp\left(\mathbf{D}_{\mathbf{c}}\right)$ or equivalently (see 6.1) that $\left(A: \Xi^{\Delta}\right)=\sharp\left(\mathbf{D}_{\mathbf{c}}\right)$. By $6.1(\mathrm{~d})$ we have $\left(A: \Xi^{\Delta}\right)=$ $\operatorname{dim}\left(E_{\mathbf{c}}\right)$. It remains to show that $\operatorname{dim}\left(E_{\mathbf{c}}\right)=\sharp\left(\mathbf{D}_{\mathbf{c}}\right)$. The left hand side is $\sum_{\lambda \in \mathfrak{o}} \operatorname{dim}\left(1_{\lambda} E_{\mathbf{c}}\right)$ where $1_{\lambda} E_{\mathbf{c}}$ is the special representation of $W_{\lambda}^{\prime}$ attached to the two-sided cell $\mathbf{c}_{\lambda}$ of $W_{\lambda}^{\prime}$ determined by $\mathbf{c}$; the right hand side is $\sum_{\lambda \in \mathfrak{o}} n_{\lambda}$ where $n_{\lambda}$ is the number of distinguished involutions of $W_{\lambda}^{\prime}$ contained in $\mathbf{c}_{\lambda}$. It is then enough to show that $\operatorname{dim}\left(1_{\lambda} E_{\mathbf{c}}\right)=n_{\lambda}$ for any $\lambda \in \mathcal{L}$ o. This can be deduced from the following known property of a two-sided cell $\mathbf{c}_{0}$ of $W_{\lambda}$ : the dimension of the special representation of $W_{\lambda}$ corresponding to $\mathbf{c}_{0}$ is equal to the number of distinguished involutions of $W_{\lambda}$ contained in $\mathbf{c}_{0}$. This completes the proof of (f) hence that of (d).

We now state the following complement to (f).

(g) If $w \cdot \lambda \in \breve{\mathbf{c}}$ and $w \cdot \lambda \notin \mathbf{D}_{\mathbf{c}}$ then $\left(A_{E_{\mathbf{c}}}: \underline{\chi}\left(\mathbb{L}_{\lambda}^{\dot{w}}\right)\right)=0$.

Let $L=\mathbb{L}_{\lambda}^{\dot{w}}$. By $6.8(\mathrm{~d})$ it is enough to show that $\left(\phi^{*}(\underline{\chi} L)\right)^{-2 a-\rho}=0$. By $6.8(\mathrm{~b}),(\mathrm{c}),\left(\phi^{*}(\underline{\chi} L)\right)^{-2 a-\rho}$ is pure of weight $-2 a-\rho$ hence it is enough to show that $\left(\phi^{*}(\underline{\chi}(L))\right)^{\{-2 a-\rho\}}=0$. Using (c) it is enough to note that, by our assumption we have $\operatorname{Hom}_{\mathcal{C}^{\mathrm{c}} Z}\left(\mathbf{1}_{0}^{\prime}, L\right)=0$.

6.10. Let $u: G \rightarrow \mathbf{p}$ be the obvious map. From [15, 7.4] we see that for $K, K^{\prime}$ in $\mathcal{M}_{m} G$ we have canonically

$$
\left(u !\left(K \otimes K^{\prime}\right)\right)^{0}=\operatorname{Hom}_{\mathcal{M}(G)}\left(\mathfrak{D}(K), K^{\prime}\right), \quad\left(u !\left(K \otimes K^{\prime}\right)\right)^{j}=0 \text { if } j>0 .
$$

We deduce that if $K, K^{\prime}$ are also pure of weight 0 then $\left(u_{!}\left(K \otimes K^{\prime}\right)\right)^{0}$ is pure of weight 0 that is $\left(u_{!}\left(K \otimes K^{\prime}\right)\right)^{0}=g r_{0}\left(u_{!}\left(K \otimes K^{\prime}\right)\right)^{0}$. From the definitions we see that we have $u_{!}\left(K \otimes K^{\prime}\right)=\phi^{*}\left(K^{\dagger} * K^{\prime}\right)$ where $K^{\dagger}$ as in 5.22. Hence for $K^{\prime}$ in $\mathcal{C}_{0}^{\mathbf{c}} G$ and $K$ in $\mathcal{C}_{0}^{\tilde{\mathbf{c}}} G$ (so that $K^{\dagger} \in \mathcal{C}_{0}^{\mathbf{c}} G$, see $5.22(\mathrm{c})$ ) we have

$$
\operatorname{Hom}_{\mathcal{M}(G)}\left(\mathfrak{D}(K), K^{\prime}\right)=\left(\phi^{*}\left(K^{\dagger} * K^{\prime}\right)\right)^{0}=\left(\phi^{*}\left(K^{\dagger} * K^{\prime}\right)\right)^{\{0\}} .
$$


Applying [24, 8.2] with $\Phi: \mathcal{D}_{\bar{m}}^{\prec} G \rightarrow \mathcal{D}_{m} \mathbf{p}, K_{1} \mapsto \phi^{*} K_{1}, c=-2 a-\rho$ (see $6.8(\mathrm{a})), K$ replaced by $K^{\dagger} * K^{\prime}$ and $c^{\prime}=2 a+\rho$ we see that we have canonically

$$
\left(\phi^{*}\left(K^{\dagger} \underline{*} K^{\prime}\right)\right)^{\{-2 a-\rho\}} \subset\left(\phi^{*}\left(K^{\dagger} * K^{\prime}\right)\right)^{\{0\}} .
$$

In particular, if $L, L^{\prime}$ are in $\mathcal{C}_{0}^{\mathbf{c}} Z$ then we have canonically

$$
\left(\phi^{*}\left(\underline{\chi}\left(L^{\prime}\right) \underline{*} \underline{\chi}(L)\right)\right)^{\{-2 a-\rho\}} \subset\left(\phi^{*}\left(\underline{\chi}\left(L^{\prime}\right) * \underline{\chi}(L)\right)\right)^{\{0\}} .
$$

Using the equality

$$
\left.\left(\phi^{*}\left(\underline{\chi}\left(L^{\prime}\right) \underline{\varkappa} \underline{\chi}(L)\right)\right)^{\{-2 a-\rho\}}=\phi^{*}\left(\underline{\chi}\left(L \underline{\bullet} \underline{\zeta}\left(\underline{\chi}\left(L^{\prime}\right)\right)\right)\right)\right)^{-2 a-\rho}
$$

which comes from 5.20(b), we deduce that we have canonically

$$
\left.\phi^{*}\left(\underline{\chi}\left(L \underline{\bullet} \underline{\zeta}\left(\underline{\chi}\left(L^{\prime}\right)\right)\right)\right)\right)^{-2 a-\rho} \subset\left(\phi^{*}\left(\underline{\chi}\left(L^{\prime}\right) * \underline{\chi}(L)\right)\right)^{\{0\}},
$$

or equivalently, using (a) with $K, K^{\prime}$ replaced by $\underline{\chi}\left(L^{\prime}\right)^{\dagger}, \underline{\chi}(L)$,

$$
\begin{aligned}
\left.\phi^{*}\left(\underline{\chi}\left(L \underline{\bullet} \underline{\zeta}\left(\underline{\chi}\left(L^{\prime}\right)\right)\right)\right)\right)^{-2 a-\rho} \subset \operatorname{Hom}_{\mathcal{C}^{\mathbf{c}} G}\left(\mathfrak{D}\left(\underline{\chi}\left(L^{\prime}\right)^{\dagger}\right), \underline{\chi}(L)\right) \\
=\operatorname{Hom}_{\mathcal{C}^{\mathrm{c}} G}\left(\mathfrak{D}\left(\underline{\chi}(L)^{\dagger}\right), \underline{\chi}\left(L^{\prime}\right)\right) .
\end{aligned}
$$

Using now $6.9(\mathrm{~d})$ with $L$ replaced by $L \underline{\boldsymbol{\bullet}} \underline{\zeta}\left(\underline{\chi}\left(L^{\prime}\right)\right)$ we deduce that we have canonically

$$
\left.\operatorname{Hom}_{\mathcal{C}^{\mathbf{c}} Z}\left(\mathbf{1}^{\prime}, L \underline{\bullet} \underline{\zeta}\left(\underline{\chi}\left(L^{\prime}\right)\right)\right)\right) \subset \operatorname{Hom}_{\mathcal{C}^{\mathbf{c}} G}\left(\mathfrak{D}\left(\underline{\chi}(L)^{\dagger}\right), \underline{\chi}\left(L^{\prime}\right)\right)
$$

or equivalently (using $4.12(\mathrm{j}))$ :

$$
\operatorname{Hom}_{\mathcal{C}^{\mathrm{c}} Z}\left(\mathfrak{D}\left(\underline{\zeta}\left(\underline{\chi}\left(L^{\prime}\right)\right)^{\dagger}\right), L\right) \subset \operatorname{Hom}_{\mathcal{C}^{\mathbf{c}} G}\left(\mathfrak{D}\left(\underline{\chi}(L)^{\dagger}\right), \underline{\chi}\left(L^{\prime}\right)\right)
$$

Now we have

$$
\begin{aligned}
\operatorname{Hom}_{\mathcal{C}^{\mathbf{c}} Z}\left(\mathfrak{D}\left(\underline{\zeta}\left(\underline{\chi}\left(L^{\prime}\right)\right)^{\dagger}\right), L\right) & =\operatorname{Hom}_{\mathcal{C}^{\tilde{c}} Z}\left(\mathfrak{D}(L), \underline{\zeta}\left(\underline{\chi}\left(L^{\prime}\right)\right)^{\dagger}\right) \\
& =\operatorname{Hom}_{\mathcal{C}^{\mathbf{c}} Z}\left((\mathfrak{D}(L))^{\dagger}, \underline{\zeta}\left(\underline{\chi}\left(L^{\prime}\right)\right)\right)
\end{aligned}
$$

hence

$$
\operatorname{Hom}_{\mathcal{C}^{\mathbf{c}} Z}\left((\mathfrak{D}(L))^{\dagger}, \underline{\zeta}\left(\underline{\chi}\left(L^{\prime}\right)\right)\right) \subset \operatorname{Hom}_{\mathcal{C}^{\mathbf{c}} G}\left(\mathfrak{D}\left(\underline{\chi}(L)^{\dagger}\right), \underline{\chi}\left(L^{\prime}\right)\right) .
$$


We set ${ }^{1} L=\mathfrak{D}\left(L^{\dagger}\right)=(\mathfrak{D}(L))^{\dagger}$ and note that

$$
\mathfrak{D}\left(\underline{\chi}(L)^{\dagger}\right)=\mathfrak{D}\left(\underline{\chi}\left(L^{\dagger}\right)\right)=\underline{\chi}\left(\mathfrak{D}\left(L^{\dagger}\right)\right)=\underline{\chi}\left({ }^{1} L\right),
$$

see $5.22(\mathrm{~d}), 5.7(\mathrm{~b})$. We obtain

$$
\operatorname{Hom}_{\mathcal{C}^{\mathrm{c}} Z}\left({ }^{1} L, \underline{\zeta}\left(\underline{\chi}\left(L^{\prime}\right)\right)\right) \subset \operatorname{Hom}_{\mathcal{C}^{\mathbf{c}} G}\left(\underline{\chi}\left({ }^{1} L\right), \underline{\chi}\left(L^{\prime}\right)\right)
$$

for any ${ }^{1} L, L^{\prime}$ in $\mathcal{C}_{0}^{\mathbf{c}} Z$.

We show that (b) is an equality:

$$
\operatorname{Hom}_{\mathcal{C}^{\mathrm{c}} Z}\left({ }^{1} L, \underline{\zeta}\left(\underline{\chi}\left(L^{\prime}\right)\right)\right)=\operatorname{Hom}_{\mathcal{C}^{\mathrm{c}} G}\left(\underline{\chi}\left({ }^{1} L\right), \underline{\chi}\left(L^{\prime}\right)\right) .
$$

Let $N^{\prime}$ (resp. $N^{\prime \prime}$ ) be the dimension of the left (resp. right) hand side of (b). It is enough to show that $N^{\prime}=N^{\prime \prime}$. We can assume that ${ }^{1} L=\mathbb{L}_{\lambda}^{\dot{w}}$, $L^{\prime}=\mathbb{L}_{\lambda^{\prime}}^{\dot{w}^{\prime}}$ where $w \cdot \lambda \in \breve{\mathbf{c}}, w^{\prime} \cdot \lambda^{\prime} \in \breve{\mathbf{c}}$. By 5.13(a), $N^{\prime}$ is the multiplicity of ${ }^{1} L$ in $\underline{\mathfrak{b}^{\prime \prime}}\left(L^{\prime}\right)$; by the fully faithfulness of $\tilde{\epsilon}$ this is the same as the multiplicity of $\tilde{\epsilon}^{1} L$ in $\tilde{\epsilon} \underline{\mathfrak{b}^{\prime \prime}}\left(L^{\prime}\right)=\underline{\mathfrak{b}^{\prime}}\left(L^{\prime}\right)=\underline{\mathfrak{b}}\left(L^{\prime}\right)$ (the last two equalities use $3.25(\mathrm{~d})$ and $3.14(\mathrm{~d}))$. By 3.13(d) this is the same as the multiplicity of $\mathbf{L}_{\lambda}^{\dot{w}}$ in

$$
\oplus_{y \in W ; y \cdot \lambda^{\prime} \in \mathbf{c}} \mathbf{L}_{\lambda^{\prime}}^{\dot{\dot{y}}} \underline{\mathbf{L}_{\lambda^{\prime}}^{\dot{w}^{\prime}}} \underline{\mathbf{L}_{y\left(\lambda^{\prime}\right)}^{\dot{y}^{-1}}}
$$

Using now 2.22(c) we see that $N^{\prime}$ is the coefficient of $t_{w \cdot \lambda}$ in

$$
\sum_{y \in W ; y \cdot \lambda^{\prime} \in \mathbf{c}} t_{y \cdot \lambda^{\prime}} t_{w^{\prime} \cdot \lambda^{\prime}} t_{y^{-1} \cdot y\left(\lambda^{\prime}\right)} \in \mathbf{H}^{\infty} .
$$

Hence if $\tau: \mathbf{H}^{\infty} \rightarrow \mathbf{Z}$ is as in 4.6 (see also 1.9 ) then

$$
N^{\prime}=\sum_{y \in W ; y \cdot \lambda^{\prime} \in \mathbf{c}} \tau\left(t_{y \cdot \lambda^{\prime}} t_{w^{\prime} \cdot \lambda^{\prime}} t_{y^{-1} \cdot y\left(\lambda^{\prime}\right)} t_{w^{-1} \cdot \lambda}\right)
$$

This can be rewritten as

$$
N^{\prime}=\sum_{y \cdot \lambda_{1} \in \mathbf{c}} \tau\left(t_{y \cdot \lambda_{1}} t_{w^{\prime} \cdot \lambda^{\prime}} t_{y^{-1} \cdot y\left(\lambda_{1}\right)} t_{w^{-1} \cdot \lambda}\right)
$$

(In the last sum, the terms corresponding to $y \cdot \lambda_{1}$ with $\lambda_{1} \neq \lambda^{\prime}$ are equal to 
zero.) By 5.6(a) we have

$$
N^{\prime \prime}=\sum_{y \cdot \lambda_{1} \in \mathbf{c}} \tau\left(t_{y^{-1} \cdot y\left(\lambda_{1}\right)} t_{w \cdot \lambda} t_{y \cdot \lambda_{1}} t_{w^{\prime-1} \cdot \lambda^{\prime}}\right) .
$$

Since $\tau\left(\xi^{b}\right)=\tau(\xi)$ for all $\xi \in \mathbf{H}^{\infty}$ and $\xi \mapsto \xi^{b}$ is the ring antiautomorphism in 1.9 we have also

$$
N^{\prime \prime}=\sum_{y \cdot \lambda_{1} \in \mathbf{c}} \tau\left(t_{w^{\prime} \cdot \lambda^{\prime}} t_{y^{-1} \cdot y\left(\lambda_{1}\right)} t_{w^{-1} \cdot \lambda} t_{y \cdot \lambda_{1}}\right) .
$$

To show that $N^{\prime}=N^{\prime \prime}$ it is enough to show that for any $y \cdot \lambda_{1} \in \mathbf{c}$ we have

$$
\tau\left(t_{y \cdot \lambda_{1}} t_{w^{\prime} \cdot \lambda^{\prime}} t_{y^{-1} \cdot y\left(\lambda_{1}\right)} t_{w^{-1 \cdot \lambda}}\right)=\tau\left(t_{w^{\prime} \cdot \lambda^{\prime}} t_{y^{-1} \cdot y\left(\lambda_{1}\right)} t_{w^{-1} \cdot \lambda} t_{y \cdot \lambda_{1}}\right)
$$

This follows by taking $\xi=t_{w^{\prime} \cdot \lambda^{\prime}} t_{y^{-1} \cdot y\left(\lambda_{1}\right)} t_{w^{-1} \cdot \lambda}, \xi^{\prime}=t_{y \cdot \lambda_{1}}$ in the identity $\tau\left(\xi \xi^{\prime}\right)=\tau\left(\xi^{\prime} \xi\right)$ which (as we see from $1.9(\mathrm{a})$ ) holds for any $\xi, \xi^{\prime}$ in $\mathbf{H}^{\infty}$. This completes the proof of the equality $N^{\prime}=N^{\prime \prime}$ and hence that of (c).

6.11. In the reminder of this section we assume that the $\mathbf{F}_{q}$-rational structure on $G$ in 2.8 is such that

(a) any $A \in C S_{\mathbf{c}}$ admits a mixed structure of pure weight 0 .

(This can be achieved by replacing if necessary $q$ by a power of $q$.)

The bifunctor $\mathcal{C}_{0}^{\mathbf{c}} G \times \mathcal{C}_{0}^{\mathbf{c}} G \rightarrow \mathcal{C}_{0}^{\mathbf{c}} G, K, K^{\prime} \mapsto K \underline{*} K^{\prime}$ in 5.20 defines a bifunctor $\mathcal{C}^{\mathbf{c}} G \times \mathcal{C}^{\mathbf{c}} G \rightarrow \mathcal{C}^{\mathbf{c}} G$ denoted again by $K, K^{\prime} \mapsto K * K^{\prime}$ as follows. Let $K \in \mathcal{C}^{\mathbf{c}} G, K^{\prime} \in \mathcal{C}^{\mathbf{c}} G$; we choose mixed structures of pure weight 0 on $K, K^{\prime}$ (this is possible by (a)), we define $K \underline{*} K^{\prime}$ as in 5.20 in terms of these mixed structures and we then disregard the mixed structure on $K \underset{*}{ } K^{\prime}$. The resulting object of $\mathcal{C}^{\mathbf{c}} G$ is denoted again by $K_{\underline{*}} K^{\prime}$; it is independent of the choices made.

In the same way the functor $\underline{\chi}: \mathcal{C}_{0}^{\mathbf{c}} Z \rightarrow \mathcal{C}_{0}^{\mathbf{c}} G$ gives rise to a functor $\mathcal{C}^{\mathbf{c}} Z \rightarrow \mathcal{C}^{\mathbf{c}} G$ denoted again by $\underline{\chi}$; the functor $\underline{\zeta}: \mathcal{C}_{0}^{\mathbf{c}} G \rightarrow \mathcal{C}_{0}^{\mathbf{c}} Z$ gives rise to a functor $\mathcal{C}^{\mathbf{c}} G \rightarrow \mathcal{C}^{\mathbf{c}} Z$ denoted again by $\underline{\zeta}$.

The operation $K \underline{*} K^{\prime}$ is again called truncated convolution. It has a canonical associativity isomorphism (deduced from that in 5.20(d)) which 
again satisfies the pentagon property. Thus $\mathcal{C}^{\mathbf{c}} G$ becomes a monoidal category; it has a braiding coming from 5.20 (a).

6.12. If $K \in \mathcal{C}^{\mathbf{c}} G$ then the isomorphisms 5.14(b) provide a central structure on $\tilde{\epsilon} \underline{\zeta}(K) \in \mathcal{C}^{\mathbf{c}} \tilde{\mathcal{B}}^{2}$ so that $\tilde{\epsilon} \zeta(K)$ can be naturally viewed as an object of $\mathcal{Z}^{\mathbf{c}}$ denoted by $\overline{\tilde{\epsilon} \zeta(K)}$. (Here $\tilde{\epsilon}$ is as in $3.3, \zeta$ is as in $5.9, \mathcal{Z}^{\mathrm{c}}$ is as in 4.9.) Then

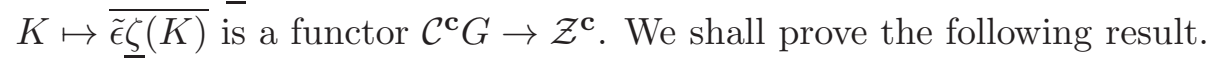

Theorem 6.13. The functor $\mathcal{C}^{\mathbf{c}} G \rightarrow \mathcal{Z}^{\mathbf{c}}, K \mapsto \overline{\tilde{\epsilon} \zeta(K)}$ is an equivalence of categories.

From 5.13(a), 3.14(d), 3.25(d) we have canonically for any $z \cdot \lambda \in \breve{\mathbf{c}}$ :

$$
\tilde{\epsilon} \zeta\left(\underline{\chi}\left(\mathbb{L}_{\lambda}^{\dot{z}}\right)\right)=\underline{\mathfrak{b}}\left(\mathbb{L}_{\lambda}^{\dot{\tilde{z}}}\right)
$$

as objects of $\mathcal{C}^{\mathbf{c}} \tilde{\mathcal{B}}^{2}$. From the definitions we see that the central structure on the left hand side of (a) provided by 6.12 is the same as the central structure on the right hand side of (a) provided by $3.14(\mathrm{j})$. Hence we have

$$
\overline{\tilde{\epsilon} \zeta\left(\underline{\chi}\left(\mathbb{L}_{\lambda}^{\dot{z}}\right)\right)}=\overline{\underline{\mathfrak{b}}\left(\mathbb{L}_{\lambda}^{\dot{z}}\right)}
$$

as objects of $\mathcal{Z}^{\mathbf{c}}$. Using this and 4.11(a) with $L^{\prime}=\tilde{\epsilon} \underline{\zeta}\left(\underline{\chi}\left(\mathbb{L}_{\lambda^{\prime}}^{\dot{w}}\right)\right)$ (where $z$. $\lambda, w \cdot \lambda^{\prime}$ are in $\breve{\mathbf{c}}$ ), we have

$$
\operatorname{Hom}_{\mathcal{C}^{c} \tilde{\mathcal{B}}^{2}}\left(\mathbf{L}_{\lambda}^{\dot{z}}, \tilde{\epsilon} \underline{\zeta}\left(\underline{\chi}\left(\mathbb{L}_{\lambda^{\prime}}^{\dot{w}}\right)\right)\right)=\operatorname{Hom}_{\mathcal{Z}^{\mathrm{c}}}\left(\overline{\tilde{\epsilon} \underline{\zeta}\left(\underline{\chi}\left(\mathbb{L}_{\lambda}^{\dot{z}}\right)\right)}, \overline{\left.\tilde{\epsilon} \zeta\left(\underline{\chi}\left(\mathbb{L}_{\lambda^{\prime}}^{\dot{w}}\right)\right)\right)}\right)
$$

Combining this with the equalities

$$
\begin{aligned}
\operatorname{Hom}_{\mathcal{C}^{\mathrm{c}} G}\left(\underline{\chi}\left(\mathbb{L}_{\lambda}^{\dot{z}}\right), \underline{\chi}\left(\mathbb{L}_{\lambda^{\prime}}^{\dot{w}}\right)\right) & =\operatorname{Hom}_{\mathcal{C}^{\mathrm{c}} Z}\left(\mathbb{L}_{l}^{\dot{z}}, \underline{\zeta}\left(\underline{\chi}\left(\mathbb{L}_{\lambda^{\prime}}^{\dot{w}}\right)\right)\right) \\
& =\operatorname{Hom}_{\mathcal{C}^{\mathrm{c}} \tilde{\mathcal{B}}^{2}}\left(\mathbf{L}_{l}^{\dot{z}}, \tilde{\epsilon} \underline{\zeta}\left(\underline{\chi}\left(\mathbb{L}_{\lambda^{\prime}}^{\dot{w}}\right)\right)\right)
\end{aligned}
$$

of which the first comes from 6.10(c) and the second comes from the fully faithfulness of $\tilde{\epsilon}$, we obtain

$$
\operatorname{Hom}_{\mathcal{C}^{c} G}\left(\underline{\chi}\left(\mathbb{L}_{\lambda}^{\dot{\tau}}\right), \underline{\chi}\left(\mathbb{L}_{\lambda^{\prime}}^{\dot{w}}\right)\right)=\operatorname{Hom}_{\mathcal{Z}^{c}}\left(\overline{\tilde{\epsilon} \underline{\zeta}\left(\underline{\chi}\left(\mathbb{L}_{\lambda}^{\dot{z}}\right)\right)}, \overline{\left.\tilde{\epsilon} \underline{\zeta}\left(\underline{\chi}\left(\mathbb{L}_{\lambda^{\prime}}^{\dot{w}}\right)\right)\right)}\right) .
$$

In other words, setting

$$
\mathbf{A}_{z \cdot \lambda, w \cdot \lambda^{\prime}}=\operatorname{Hom}_{\mathcal{C}^{c} G}\left(\underline{\chi}\left(\mathbb{L}_{\lambda}^{\dot{z}}\right), \underline{\chi}\left(\mathbb{L}_{\lambda^{\prime}}^{\dot{w}}\right)\right),
$$




$$
\left.\mathbf{A}_{z \cdot \lambda, w \cdot \lambda^{\prime}}^{\prime}=\operatorname{Hom}_{\mathcal{Z}} \overline{(\overline{\tilde{\epsilon} \zeta}(\underline{\chi}(\mathbb{L} \dot{\dot{\tau}}))}, \overline{\tilde{\epsilon} \underline{\zeta}\left(\underline{\chi}\left(\mathbb{L}_{\lambda^{\prime}}^{\dot{w}}\right)\right)}\right),
$$

we have

$$
\mathbf{A}_{z \cdot \lambda, w \cdot \lambda^{\prime}}=\mathbf{A}_{z \cdot \lambda, w \cdot \lambda^{\prime}}^{\prime}
$$

Note that the identification (c) is induced by the functor $K \mapsto \overline{\tilde{\epsilon} \zeta(K)}$. Let $\mathbf{A}=\oplus \mathbf{A}_{z \cdot \lambda, w \cdot \lambda^{\prime}}, \mathbf{A}^{\prime}=\oplus \mathbf{A}_{z \cdot \lambda, w \cdot \lambda^{\prime}}$ (both direct sums are taken over all $z$. $\lambda, w \cdot \lambda^{\prime}$ in $\breve{\mathbf{c}}$ ). Then from (c) we have $\mathbf{A}=\mathbf{A}^{\prime}$. Note that this identification is compatible with the obvious algebra structures of $\mathbf{A}, \mathbf{A}^{\prime}$.

For any $A \in C S_{\mathbf{c}}$ we denote by $\mathbf{A}_{A}$ the set of all $f \in \mathbf{A}$ such that for any $z \cdot \lambda, w \cdot \lambda^{\prime}$, the $\left(z \cdot \lambda, w \cdot \lambda^{\prime}\right)$-component of $f$ maps the $A$-isotypic component of $\underline{\chi}\left(\mathbb{L}_{\lambda}^{\dot{z}}\right)$ to the $A$-isotypic component of $\underline{\chi}\left(\mathbb{L}_{\lambda^{\prime}}^{\dot{w}}\right)$ and any other isotypic component of $\underline{\chi}\left(\mathbb{L}_{\lambda}^{\dot{z}}\right)$ to 0 . Thus, $\mathbf{A}=\oplus_{A \in C S_{\mathbf{c}}} \mathbf{A}_{A}$ is the decomposition of $\mathbf{A}$ into a sum of simple algebras. (Each $\mathbf{A}_{A}$ is nonzero since, by $5.2(\mathrm{c})$ and $5.5(\mathrm{a})$, any $A$ is a summand of some $\underline{\chi}\left(\mathbb{L}_{\lambda}^{\dot{z}}\right)$.)

From [28], 44] we see that $\mathcal{Z}^{\mathbf{c}}$ is a semisimple abelian category with finitely many simple objects up to isomorphism. Let $\mathfrak{S}$ be a set of representatives for the isomorphism classes of simple objects of $\mathcal{Z}^{\mathbf{c}}$. For any $\sigma \in \mathfrak{S}$ we denote by $\mathbf{A}_{\sigma}^{\prime}$ the set of all $f^{\prime} \in \mathbf{A}^{\prime}$ such that for any $z \cdot \lambda, w \cdot \lambda^{\prime}$, the $\left(z \cdot \lambda, w \cdot \lambda^{\prime}\right)$-component of $f^{\prime}$ maps the $\sigma$-isotypic component of $\overline{\tilde{\epsilon} \zeta\left(\underline{\chi}\left(\mathbb{L} \tilde{L}_{\lambda}^{\dot{z}}\right)\right)}$ to the $\sigma$-isotypic component of $\left.\overline{\tilde{\epsilon} \zeta\left(\underline{\chi}\left(\mathbb{L}_{\lambda^{\prime}}^{\dot{w}}\right)\right)}\right)$ and all other isotypic components of $\overline{\tilde{\epsilon}} \underline{\zeta}\left(\underline{\chi}\left(\mathbb{L}_{\lambda}^{\dot{z}}\right)\right)$ to zero. Then $\mathbf{A}^{\prime}=\oplus_{\sigma} \mathbf{A}_{\sigma}^{\prime}$ is the decomposition of $\mathbf{A}^{\prime}$ into a sum of simple algebras. (Each $\mathbf{A}_{\sigma}^{\prime}$ is nonzero since any $\sigma$ is a summand of some $\overline{\tilde{\epsilon} \underline{\zeta}\left(\underline{\chi}\left(\mathbb{L}_{\lambda}^{\dot{x}}\right)\right)}$. Indeed, we can find $x \cdot \lambda \in \mathbf{c}$ such that $\mathbf{L}_{\lambda}^{\dot{x}}$ is a summand of $\sigma$, viewed as an object of $\mathcal{C}^{\mathbf{c}} \tilde{\mathcal{B}}^{2}$, by $4.9(\mathrm{a}), \sigma$ is a summand of $\overline{I\left(\mathbf{L}_{\lambda}^{\dot{x}}\right)}$. If in addition, $x \cdot \lambda \in W \mathfrak{s}$ then, by (a), $\sigma$ is a summand of $\tilde{\epsilon} \underline{\zeta}\left(\underline{\chi}\left(\mathbb{L}_{\lambda}^{\dot{x}}\right)\right)$, as required. If $x \cdot \lambda \notin W \mathfrak{s}$ then, by 4.9 (b) we have $I\left(\mathbf{L}_{\lambda}^{\dot{x}}\right) \neq 0$ which is a contradiction.)

Since $\mathbf{A}=\mathbf{A}^{\prime}$, from the uniqueness of decomposition of a semisimple algebra as a direct sum of simple algebras, we see that there is a unique bijection $C S_{\mathbf{c}} \leftrightarrow \mathfrak{S}, A \leftrightarrow \sigma_{A}$ such that $\mathbf{A}_{A}=\mathbf{A}_{\sigma_{A}}^{\prime}$ for any $A \in C S_{\mathbf{c}}$. From the definitions we now see that for any $A \in C S_{\mathrm{c}}$ we have $\overline{\tilde{\epsilon} \zeta(K)} \cong \sigma_{A}$. Therefore, Theorem 6.13 holds. 
Theorem 6.14. Let $L \in \mathcal{C}^{\mathbf{c}} Z, K \in \mathcal{C}^{\mathbf{c}} G$. We have canonically

$$
\operatorname{Hom}_{\mathcal{C}^{\mathbf{c}} Z}(L, \underline{\zeta}(K))=\operatorname{Hom}_{\mathcal{C}^{\mathbf{c}} G}(\underline{\chi}(L), K)
$$

We can assume that $L=\mathbb{L}_{\lambda}^{\dot{z}}$ where $z \cdot \lambda \in \breve{\mathbf{c}}$. From 6.13 and its proof we see that

$$
\operatorname{Hom}_{\mathcal{C}^{\mathbf{c}} G}(\underline{\chi}(L), K)=\operatorname{Hom}_{\mathcal{Z}^{\mathrm{c}}}(\overline{\overline{\tilde{\epsilon}} \underline{\zeta}(\underline{\chi}(L))}, \overline{\tilde{\epsilon} \underline{\zeta}(K)})=\operatorname{Hom}_{\mathcal{Z}^{\mathrm{c}}}\left(\overline{I\left(\mathbf{L}_{\lambda}^{\dot{z}}\right)}, \overline{\tilde{\epsilon} \underline{\zeta}(K)}\right) .
$$

Using 4.9(a) we see that

$$
\operatorname{Hom}_{\mathcal{Z}^{\mathrm{c}}}\left(\overline{I\left(\mathbf{L}_{\lambda}^{\dot{z}}\right)}, \overline{\tilde{\epsilon} \zeta(K)}\right)=\operatorname{Hom}_{\mathcal{C}^{\mathbf{c}} \tilde{\mathcal{B}}^{2}}\left(\mathbf{L}_{\lambda}^{\dot{z}}, \tilde{\epsilon} \underline{\zeta}(K)\right)=\operatorname{Hom}_{\mathcal{C}^{\mathbf{c}} Z}(L, \underline{\zeta}(K)) .
$$

This proves the theorem.

6.15. We show that for $K \in \mathcal{C}^{\mathbf{c}} G$ we have canonically

$$
\mathfrak{D}(\underline{\zeta}(\mathfrak{D}(K)))=\underline{\zeta}(K) .
$$

Here the first $\underline{\zeta}$ is relative to $\tilde{\mathbf{c}}$. It is enough to show that for any $L \in \mathcal{C}^{\mathbf{c}} Z$ we have canonically

$$
\operatorname{Hom}_{\mathcal{C}^{\mathbf{c}} Z}(L, \mathfrak{D}(\underline{\zeta}(\mathfrak{D}(K))))=\operatorname{Hom}_{\mathcal{C}^{\mathbf{c}} Z}(L, \underline{\zeta}(K)) .
$$

Here the left side equals

$$
\begin{gathered}
\operatorname{Hom}_{\mathcal{C}^{\tilde{c}} Z}(\underline{\zeta}(\mathfrak{D}(K)), \mathfrak{D}(L))=\operatorname{Hom}_{\mathcal{C}^{\mathbf{c}} G}(\mathfrak{D}(K), \underline{\chi}(\mathfrak{D}(L))) \\
=\operatorname{Hom}_{\mathcal{C}^{\mathbf{c}} G}(\mathfrak{D}(K), \mathfrak{D}(\underline{\chi}(L))) .
\end{gathered}
$$

(We have used 6.14(a) for $\tilde{\mathbf{c}}$ and 5.7(b).) The right hand side equals

$$
\operatorname{Hom}_{\mathcal{C}^{\mathbf{c}} G}(\underline{\chi}(L), K)=\operatorname{Hom}_{\mathcal{C}^{\mathbf{c}} G}(\mathfrak{D}(K), \mathfrak{D}(\underline{\chi}(L))) .
$$

(We have again used 6.14(a).) This proves (a).

6.16. The monoidal structure on $\mathcal{C}^{\mathbf{c}} \tilde{\mathcal{B}}^{2}$ induces a monoidal structure on $\mathcal{Z}^{\mathbf{c}}$. Using 5.21(b) and 3.24(b) we see that the equivalence of categories in 6.13 is compatible with the monoidal structures. Since $\mathcal{Z}^{\mathbf{c}}$ has a unit object, it 
follows that the monoidal category $\mathcal{C}^{\mathbf{c}} G$ also has a unit object, say $A$. We show:

$$
A \cong A_{E_{\mathbf{c}}}
$$

with $A_{E_{\mathbf{c}}}$ as in 6.8(c). From 6.9(f), (g) we see that for $w \cdot \lambda \in \breve{c},\left(A_{E_{\mathbf{c}}}: \underline{\chi}\left(\mathbb{L}_{\lambda}^{\dot{w}}\right)\right)$ is 1 if $w \cdot \lambda \in \mathbf{D}_{\mathbf{c}}$ and is 0 if $w \cdot \lambda \notin \mathbf{D}_{\mathbf{c}}$. Using 6.13 we deduce that

$$
\operatorname{dim} \operatorname{Hom}_{\mathcal{C}^{\mathbf{c}} \tilde{\mathcal{B}}^{2}}\left(\mathbf{L}_{\lambda}^{\dot{w}}, \tilde{\epsilon} \underline{\zeta}\left(A_{E_{\mathbf{c}}}\right)\right)
$$

is 1 if $w \cdot \lambda \in \mathbf{D}_{\mathbf{c}}$ and is 0 if $w \cdot \lambda \notin \mathbf{D}_{\mathbf{c}}$. Thus $\tilde{\epsilon} \zeta\left(A_{E_{\mathbf{c}}}\right)$ is isomorphic in $\mathcal{C}^{\mathbf{c}} \tilde{\mathcal{B}}^{2}$ to the unit object $\mathbf{1}$ of the monoidal category $\mathcal{C}^{\mathbf{c}} \tilde{\mathcal{B}}^{2}$. Then $\tilde{\epsilon} \underline{\zeta}\left(A_{E_{\mathbf{c}}}\right)$ viewed as an object of $\mathcal{Z}^{\mathbf{c}}$ is also the unit object of $\mathcal{Z}^{\mathbf{c}}$ hence is isomorphic in $\mathcal{Z}^{\mathbf{c}}$ to $\tilde{\epsilon} \underline{\zeta}(A)$. Using 6.13 we deduce that (a) holds.

\section{Acknowledgment}

This research was supported in part by National Science Foundation grant DMS-1303060 and by a Simons Fellowship. A part of the writing was done during a visit (April, May 2015) to the Mittag-Leffler Institute, Djursholm, Sweden, whose hospitality and support is hereby acknowledged.

\section{References}

1. A. Beilinson, J. Bernstein and P. Deligne, Faisceaux pervers, Astérisque, 100 (1982).f

2. R. Bezrukavnikov, M. Finkelberg and V. Ostrik, Character D-modules via Drinfeld center of Harish-Chandra bimodules, Invent. Math., 188 (2012), 589-620.

3. P. Deligne and G. Lusztig, Representations of reductive groups over finite fields, Ann. Math., 103 (1976), 103-161.

4. P. Etingof, D. Nikshych and V. Ostrik, On fusion categories, Ann. Math., 162 (2005), 581-642.

5. M. Geck and G. Pfeiffer, Characters of Finite Coxeter Groups and Iwahori-Hecke Algebras, Clarendon Press, Oxford, 2000.

6. A. Joyal and R. Street, Tortile Yang-Baxter operators in tensor categories, J. Pure Appl. Alg., 71 (1991), 43-51.

7. D. Kazhdan and G. Lusztig, Representations of Coxeter groups and Hecke algebras, Invent. Math., 53 (1979), 165-184. 
8. G. Lusztig, Representations of Finite Chevalley Groups, CBMS regional conference series in Math., 39, Amer. Math. Soc., 1878.

9. G. Lusztig, On the Reflection Representation of a Finite Chevalley Group, Representation theory of Lie groups, LMS Lecture Notes Ser., 34 (1979), Cambridge U. Press.

10. G. Lusztig, A class of irreducible representations of a Weyl group, Proc. Kon. Nederl. Akad., Ser. A, 82 (1979), 323-335.

11. G. Lusztig, A class of irreducible representations of a Weyl group, II, Proc. Kon. Nederl. Akad. Ser. A, 85 (1982), 219-226.

12. G. Lusztig, Green polynomials and singularities of unipotent classes, Adv. Math., 42 (1981), 169-178.

13. G. Lusztig, Characters of reductive groups over a finite field, Ann. Math. Studies 107, Princeton Univ. Press, 1984.

14. G. Lusztig, Character sheaves I, Adv. Math., 56 (1985), 193-237.

15. G. Lusztig, Character sheaves II, Adv. Math., 57 (1985), 226-265.

16. G. Lusztig, Character sheaves III, Adv. Math., 57 (1985), 266-315.

17. G. Lusztig, Character sheaves IV, Adv. Math., 59 (1986), 1-63.

18. G. Lusztig, Hecke Algebras with Unequal Parameters, CRM Monograph Ser. 18, Amer. Math. Soc., 2003.

19. G. Lusztig, Character sheaves on disconnected groups VI, Represent. Th., 8 (2004), 377-413.

20. G. Lusztig, Character sheaves on disconnected groups VII, Represent. Th., 9 (2005), 209-266.

21. G. Lusztig, Character sheaves on disconnected groups IX, Represent. Th., 10 (2006), 353-379.

22. G. Lusztig, Character sheaves on disconnected groups X, Represent. Th., 13 (2009), 82-140.

23. G. Lusztig, On the cleanness of cuspidal character sheaves, Moscow Math. J., 12 (2012), 621-631.

24. G. Lusztig, Truncated convolution of character sheaves, Bull. Inst. Math. Acad. Sin. (N.S.), 10 (2005), 1-72.

25. G. Lusztig, Unipotent representations as a categorical centre, Represent. Th., 19 (2015), 211-235.

26. S. Majid, Representations, duals and quantum doubles of monoidal categories, Rend. Circ. Mat. Palermo, 26 (1991), 197-206.

27. M. Müger, From subfactors to categories and topology I. Frobenius algebras in and Morita equivalence of tensor categories, J. Pure Appl. Alg., 180 (2003), 81-157. 
28. M. Müger, From subfactors to categories and topology II. The quantum double of tensor categories and subfactors, J. Pure Appl. Alg., 180 (2003), 159-219.

29. N. Spaltenstein, On the generalized Springer correspondence for exceptional groups, Algebraic Groups and Related Topics (R. Hotta, Ed.), Advanced Studies in Pure Math., 6 (1985), North Holland, Kinokunia.

30. T. Yokonuma, Sur la structure des anneaux de Hecke d'un groupe de Chevalley fini, C. R. Acad. Sci. Paris Ser. A, 264 (1967), A334-A347. 